Florida International University FIU Digital Commons

FIU Electronic Theses and Dissertations

University Graduate School

$5-21-2015$

\title{
A Charged Fusion Product Diagnostic for a Spherical Tokamak
}

\author{
Ramona V. Perez \\ Florida International University, rvale006@fiu.edu
}

DOI: 10.25148 /etd.FIDC000066

Follow this and additional works at: https://digitalcommons.fiu.edu/etd

Part of the Engineering Physics Commons, and the Other Physics Commons

\section{Recommended Citation}

Perez, Ramona V., "A Charged Fusion Product Diagnostic for a Spherical Tokamak" (2015). FIU Electronic Theses and Dissertations. 2233.

https://digitalcommons.fiu.edu/etd/2233

This work is brought to you for free and open access by the University Graduate School at FIU Digital Commons. It has been accepted for inclusion in FIU Electronic Theses and Dissertations by an authorized administrator of FIU Digital Commons. For more information, please contact dcc@fiu.edu. 


\title{
FLORIDA INTERNATIONAL UNIVERSITY
}

Miami, Florida

\section{A CHARGED FUSION PRODUCT DIAGNOSTIC FOR A SPHERICAL TOKAMAK}

\author{
A dissertation submitted in partial fulfillment of the \\ requirements for the degree of \\ DOCTOR OF PHILOSOPHY \\ in \\ PHYSICS \\ by \\ Ramona Leticia Valenzuela Perez
}


To: Dean Michael Heithaus

College of Arts and Sciences

This dissertation, written by Ramona Leticia Valenzuela Perez, and entitled A Charged Fusion Product Diagnostic for a Spherical Tokamak, having been approved in respect to style and intellectual content, is referred to you for judgement.

We have read this dissertation and recommend that it be approved.

$\begin{array}{r}\hline \text { Douglass S. Darrow } \\ \hline \text { Oren V. Maxwell } \\ \hline \text { Richard A. Bone } \\ \hline \text { Michael C. Sukop } \\ \hline \text { Werner U. Boeglin, Major Professor }\end{array}$

Date of Defense: May 21, 2015

The dissertation of Ramona Leticia Valenzuela Perez is approved.

Dean Michael Heithaus

College of Arts and Sciences

Dean Lakshmi N. Reddi

University Graduate School

Florida International University, 2015 
(C) Copyright 2015 by Ramona Leticia Valenzuela Perez

All rights reserved. 


\section{DEDICATION}

I would like to dedicate this dissertation to my grandparents. 


\section{ACKNOWLEDGMENTS}

I would like to thank my advisor, Werner Boeglin, for all of his efforts in my development as a researcher. I thank my dissertation committee: Douglass Darrow, Oren Maxwell, Jorge Rodriguez, Michael Sukop, and Richard Bone for their input and dedication throughout my final years of graduate research. I also appreciate the support I have received from collaborators from the Plasma Physics Laboratory and the Culham Centre for Fusion Energy (CCFE) especially: Scott Allan, Ken McClements, Marco Cecconnello, Nigel ThomasDavies, Bob Kaita, Brent Stratton, Lane Roquemore, Rob Akers, Sergei Sharapov, Vasili Kiptily, Iwona Klimek, and John Schmitt.

I also acknowledge encouragement, professional guidance, and research advice I have received from former and present FIU Professors throughout my undergraduate and graduate career: James Fourqurean, Laird Kramer, Wenzhi Li, Jin He, Joerg Reinhold, Misak Sargsian, Brian Raue, Jeff Saul, Walter Van Hamme, Xuewen Wang, James Webb, Lei Guo, and Jiandi Zhang. I would like to thank former and present Physics Department Staff for providing administrative support for my research efforts: Robert Brown, Ofelia Fernandez, Maria B. Martinez, Jeannie Carmona, Vanessa Pons, Chelonda Walker, Omar Tolbert, and Elizabeth Bergano-Smith. I also want to thank FIU Physics Department Staff for providing technical and engineering support for my research and teaching efforts: Carlos Orta, Barry Branch, Norman Huang, and Manuel Wong.

I thank CCFE staff for providing administrative and logistical support for my research efforts: Kathy Patton, Robert Franklin, Nick Holloway, Andy Butler, and the security guards. Moreover, I thank CFFE staff for providing technical and engineering support for my research efforts: Jake Ashton, Tony Owen, Rory Conway, Alex Angell, Ian Fitzergald, Ivan Lupelli, John Storrs, David Langridge, Pete Edney, Neil Reed, Garry O’ Sullivan, Brian Lloyd, Ashley Stevens, Paul Stevenson, Robert Stephens, Stuart Bray, Mike 
Driscoll, Martin Simmonds, Geoff Lee, Stuart Green, Rob Gaffka, the PAT testers, and the MAST team. I also thank Florida State University collaborators for providing logistical and technical support for my research efforts: Ingo Wiedenhver and Lagy Baby.

I would like to thank several former and present FIU undergraduate and graduate students who have positively contributed to my graduate experience, both through research and mentoring opportunities: Adrianna Angulo, Pierre Avila, Javiera LaTorre, Omar Leon, Carlos Lievano, Carlos Lopez, Doug Tuckler, and Alex Netepenko. I also appreciate support from graduate colleagues: Trisha Ashley, Carlos Granadaos, Yuehai Yang, Suman Neupane, Anirbaan Mukherjee, Richard Galvez, and Namuna Panday.

I would like to thank my undergraduate funding sources, without which I would have never continued to graduate school: Miccosukee Fellowship for Everglades Research, Marsh Scholarship, FIU Ronald E. McNair Baccalaureate Program, FIU Presidential Scholarship, FIU Salutatorian Scholarship, Florida Bright Futures Scholarship, and Florida Department of Transportation Employee Dependent Scholarship. I would also like to thank funding sources which have helped me complete my graduate degree: American Physical Society Forum on Graduate Student Affairs Travel Award for Excellence in Graduate Research, FIU Graduate \& Professional Student Committee (GPSC) Conference Grant, FIU GPSC Research Grant, FIU University Graduate School Dissertation Year Fellowship, FIU College of Arts \& Sciences Travel Support, FIU Ronald E. McNair Baccalaureate Program, Research Assistantships through the U.S. Department of Energy Contract Numbers: DESC0001157 \& DEAC0209CH11466, and Teaching Assistantships through the FIU Physics Department and FIU University Graduate School.

I thank the American Institute of Physics Publishing and the Review of Scientific Instruments for copyright permissions. I also recognize the CCFE Mega Amp Spherical Tokamak 
(MAST) Data Access Policy which grants me the use of MAST data in my dissertation with a statement that the MAST data used (excluding cited published data) is given preliminary status.

Finally, I would like to thank my family for encouragement and moral support, especially my husband Gerson Perez, my parents Linda and Cholo Valenzuela, and my sister Chonti Sauder. I owe my continual professional development throughout my undergraduate and graduate career to my father, Cholo Valenzuela. 
ABSTRACT OF THE DISSERTATION

A CHARGED FUSION PRODUCT DIAGNOSTIC FOR A SPHERICAL TOKAMAK

by

Ramona Leticia Valenzuela Perez

Florida International University, 2015

Miami, Florida

Werner U. Boeglin, Major Professor

Designs for future nuclear fusion power reactors rely on the ability to create a stable plasma (hot ionized gas of hydrogen isotopes) as a medium with which to sustain nuclear fusion reactions. My dissertation work involves designing, constructing, testing, installing, operating, and validating a new diagnostic for spherical tokamaks, a type of reactor test facility. Through detecting charged particles emitted from the plasma, this instrument can be used to study fusion reaction rates within the plasma and how they are affected by plasma perturbations. Quantitatively assessing nuclear fusion reaction rates at specific locations inside the plasma and as a function of time can provide valuable data that can be used to evaluate theory-based simulations related to energy transport and plasma stability.

The Proton Detector (PD), installed in the Mega Amp Spherical Tokamak (MAST) at the Culham Centre for Fusion Energy (CCFE) in Abingdon, England, was the first instrument to experimentally detect $3 \mathrm{MeV}$ Protons and $1 \mathrm{MeV}$ Tritons created from deuteriumdeuterium (hydrogen isotopes) nuclear fusion reactions inside a spherical tokamak's plasma. The PD consists of an array of particle detectors with a protective housing and the necessary signal conditioning electronics and readout. After several years of designing (which included simulations for detector orientations), fabricating, and testing the PD, it was in- 
stalled in MAST and data were collected over a period of two months in the summer of 2013. Proton and triton rates as high as $200 \mathrm{kHz}$ were measured and an initial radial profile of these fusion reaction rates inside the plasma was extracted.

These results will be compared to a complementary instrument at MAST as well as theory-based simulations and form the knowledge basis for developing a larger future instrument. The design and performance of all instrument components (electrical, computational, mechanical), and subsequent data analysis methods and results are described in this dissertation. 


\section{TABLE OF CONTENTS}

CHAPTER

PAGE

1. INTRODUCTION AND MOTIVATION 1

$1.1 \quad$ Plasma Properties in Fusion Research . . . . . . . . . . . . . . . . . . 1

1.2 Producing Plasmas for Fusion Research . . . . . . . . . . . . . . . . 5

1.3 Plasma Instabilities . . . . . . . . . . . . . . . . . . . . . . . . . . . .

1.4 Methods to Study Plasma Instabilities . . . . . . . . . . . . . . . . . 19

1.5 Dissertation Research Overview . . . . . . . . . . . . . . . . 28

2. DIAGNOSTIC MECHANICAL DESIGN 30

$2.1 \quad$ Orbit Code . . . . . . . . . . . . . . . . . 30

2.2 General Design Constraints . . . . . . . . . . . . . . . . . 39

2.3 Mechanical Housing Design . . . . . . . . . . . . . . . 42

3. DIAGNOSTIC ELECTRICAL DESIGN 50

3.1 Data Acquisition System Design . . . . . . . . . . . . . . . . 50

3.2 Detector Signals . . . . . . . . . . . . . . . . 51

3.3 Signals Traveling Through the RP . . . . . . . . . . . . . . . 52

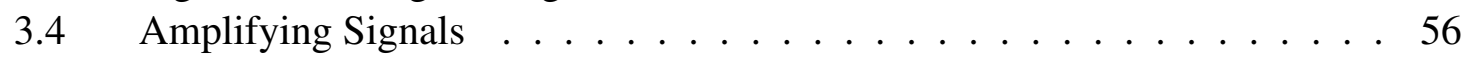

3.5 Data Acquisition Electronics . . . . . . . . . . . . . . . . 57

3.6 Data Acquisition Software . . . . . . . . . . . . 61

4. EXPERIMENT AND DATA COLLECTION 67

4.1 Plasma Pulse Characteristics . . . . . . . . . . . . . 67

4.2 Data Collection . . . . . . . . . . . . . . . . 68

4.3 RP Positions . . . . . . . . . . . . . . . . . 71

4.4 Detector Response to Varying Particle Rates . . . . . . . . . . . . . . . 74

5. DATA ANALYSIS $\quad 82$

$5.1 \quad$ First Step: Fit Particle Pulses _. . . . . . . . . . . . . . . . 82

5.2 Second Step: Create Energy Spectra and Production Rates . . . . . . . . . 93

5.3 Mid-plane Radii . . . . . . . . . . . . . . . . . 101

6. RESULTS AND DISCUSSION 103

6.1 Ion Relaxation Times . . . . . . . . . . . . . . . 103

6.2 Proton Production Rates . . . . . . . . . . . . . . . . . 117

6.3 Proton Emission Profile . . . . . . . . . . . . . . 128

7. SUMMARY 142

7.1 Charged Fusion Product Diagnostic . . . . . . . . . . . . . . . . . . 142

7.2 Future Diagnostic Development . . . . . . . . . . . . . 144 


\section{LIST OF REFERENCES}




\section{LIST OF TABLES}

TABLE

PAGE

1.1 This table contains several aspect ratios for several tokamaks.[1][3][6][36]

1.2 This table contains characteristic values for the toroidal magnetic field and current used for different tokamaks to confine plasmas.[36][6][1][3] . . . . 9

1.3 This table contains characteristic values for the neutral beam heating power for several tokamaks.[36][6][1][3] . . . . . . . . . . . . . . . . . 12

2.1 This table contains calculated characteristic values for products from DD reactions.[58] . . . . . . . . . . . . . . . . . 34

2.2 This table contains values for the gyro-radii of protons, tritons, and helium3 ions.[58] . . . . . . . . . . . . . . . . . . . 35

4.1 This table lists individual channel (and detector) angular orientations within the PD coordinate system. . . . . . . . . . . . . . . . . . . 73

4.2 This table lists individual channel (and detector) collimator entrance distances relative to the center of the tokamak. . . . . . . . . . . 73

6.1 This table summarizes the time decay constants for protons, $\tau_{1}$ and $\tau_{2}$, during shots in which two subsequent NBIs take place. $\mathrm{R}$ refers to the mid-plane radius value for each channel. Background noise in the waveform data prevented the calculation of proton rates, and therefore time decay constants, for a few channels. . . . . . . . . . . . . . . 112

6.2 This table summarizes the time decay constants for neutrons, $\tau_{1}$ and $\tau_{2}$, during shots in which two subsequent NBIs take place. The fission chamber instrument is represented by FC. . . . . . . . . . . . . . . . . . 112 


\section{LIST OF FIGURES}

FIGURE

PAGE

1.1 A color composite image of a typical plasma in the Mega Amp Spherical Tokamak (MAST) fusion device at the Culham Centre for Fusion

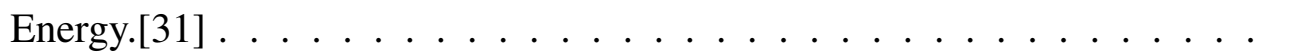

1.2 Charged particles moving along magnetic field lines will rotate, or gyrate, in a direction perpendicular to the magnetic field. The resulting path, or trajectory, of the particle is helical. The mass and charge of the particle will determine the direction and the size of gyration.[40] . . . . . . .

1.3 The reactivity (proportional to the experimental cross section) for several nuclear fusion reactions are displayed above. The relationship between temperature and the probability of nuclear fusion reactions occurring provides the motivation for fusion plasma operating temperatures.[33] . . . . 5

1.4 The above image is qualitative. Different tokamaks have different aspect ratios, or ratios of their major radii to minor radii. The aspect ratio can be calculated using the expression $A_{m}=R_{m} / r_{m}$. The original figure is from the Culham Centre of Fusion Energy.[10]. . . . . . . . . . . . . 6

1.5 A cross-sectional view (CAD drawing) showing the Mega Amp Spherical Tokamak (MAST) at the Culham Centre for Fusion Energy in Abingdon, the United Kingdom.[35] . . . . . . . . . . . . . . . . . . .

1.6 Three main methods are used to heat a plasma to operating temperature, a temperature high enough to sustain deuterium-deuterium nuclear fusion reactions. The first method is ohmic heating, which is followed by a combination of radio frequency heating and the injection of an energetic neutral beam. [38]. . . . . . . . . . . . . . . . . . . .

1.7 A tokamak uses horizontal, or toroidal, magnetic field lines to confine a plasma in the horizontal direction. It also uses vertical, or poloidal, magnetic field lines to confine a plasma in the vertical direction. Together, the resultant magnetic field lines that confine the plasma are twisted, or helical.[32] . . . . . . . . . . . . . . . . .

1.8 This diagrams shows the basic process of creating a beam of energetic neutral atoms for the Joint European Torus (JET).[39] . . . . . . . . . . . 10 
1.9 During a nuclear fusion reaction inside of a plasma, when two deuterium atoms fuse, they can create either a proton $(\mathrm{P})$ and a triton or a neutron $(\mathrm{N})$ and a helium-3 ion. . . . . . . . . . . . . . . . . 12

1.10 Both branches of a DD fusion reaction, (1.9) and (1.10), have approximately the same cross section. Therefore, they each have approximately the same probability of occurring $(\sim 50 \%)$. At $70 \mathrm{keV}$ the experimental cross section for the $d(d, n) h e 3$ and $d(d, p) t$ branch are 0.0091 barns (unit of area) and 0.0088 barns, respectively. Data used to make the graph are from the Evaluated Nuclear Data File (ENDF) Database.[11] . . . . . . . 13

1.11 The differential cross section for both DD fusion reaction branches shows anisotropy. The anisotropy can vary in range depending on the reaction branch and the initial deuteron energy (E). The constants $A_{0}, A_{1}$, and $A_{2}$ change depending on $\mathrm{E}$ and the reaction branch. The units for $\sigma$ are millibarns (mb) per steradian ( $\mathrm{sr}$ ). Data and the form of $\sigma(\theta)$ used to make the graph are from the Lawrence Livermore National Laboratory.[8] . . . . 14

1.12 The above images are qualitative. The image on the left displays nested flux surfaces for a high aspect ratio plasma, while the image on the right displays nested flux surfaces for a low aspect ratio (such as that created by a spherical tokamak). Magnetic field lines travel along the flux surfaces. The flux surfaces are characterized by constant pressure. . . . . . . . . . . 15

1.13 The energy confinement time is related to balancing the energy lost from the plasma and the external heating power supplied to the plasma.[41] . . . 18

1.14 This graph displays the Lawson Criterion for nuclear fusion reactions between hydrogen isotopes and helium isotopes. A tokamak that is able to satisfy conditions above the plotted curves can reach ignition.[34] . . . . . 20

1.15 The above qualitative image represents a plasma created by a conventional tokamak. Charged particles gyrating along helical magnetic field lines in a toroidal plasma can leave the plasma and hit a detector if the gyro-radii of their trajectories are large enough.[37] . . . . . . . . . . . . . . . 21

1.16 The above qualitative image is of nested constant flux surfaces within a plasma created by a spherical tokamak. Charged particles gyrating along helical magnetic field lines in a spherically-shaped toroidal plasma can leave the plasma and hit a detector if the gyro-radii of their trajectories are large enough. The red inner surface represents the hot central region of the plasma. . . . . . . . . . . . . . . . . . 21 
1.17 Proton detection techniques allow for a relatively smaller and lightweight mechanical housing design when compared with neutron detection techniques. Note that a scaled CAD drawing of the PD diagnostic was Photoshopped into the above image and used with approximations for the weight and dimensions of the instruments to provide a qualitative reference. 25

1.18 Above is a qualitative image with a projection of Proton Detector and Neutron Camera toroidal sightlines at the Mega Amp Spherical Tokamak (MAST). The original image is from Cecconello et al.'s work (2012).[19] . 26

2.1 Figures a) through d) in the images above are qualitative. Figure a) is a cross-sectional three-dimensional view of nested constant flux surfaces within the plasma. Figure b) is a two-dimensional vertical, or poloidal, slice of the flux surfaces. Results from the EFIT code provide information about this poloidal flux. Figure c) is the simulated two-dimensional poloidal particle emissivity, $\mathrm{S}$, for regions in the plasma. This example $\mathrm{S}$ has an elliptical shape because it is based on a gaussian function, where the color red represents the highest emission and blue represents the lowest emission. Figure d) combines the poloidal flux in b) and the particle emissivity in c). Figure e) is an example of the orbit's code graphical output in which poloidal particle orbits are plotted over d). The poloidal coordinates of the graphical output are $\mathrm{R}$ ( $\mathrm{m}$ from the center of the tokamak) and $\mathrm{z}$ (m above and below the plasma mid-plane). . . . . . . . . . 33

2.2 The above images are graphical outputs of the orbit code which display particle orbits. Figure a), from Perez et al., Rev. Sci. Instrum. 85, 11 D701 (2014), displays the poloidal projection of the central orbits plotted over the poloidal flux and emission regions (coordinates of R and z).[55] Figure b) shows the corresponding thickness of the orbit bundle incident on the detector channels. Figure c) displays the toroidal projection of the central orbits (coordinates of $\mathrm{X}$ and $\mathrm{Y}$ ). Figure d) shows the corresponding thickness of the orbit bundle incident on the detector channels. The thickness of the orbit bundle is directly associated to the collimator opening in front of the detector. . . . . . . . . . . . . . . . . . . . 36

2.3 The above images are qualitative. A range of trajectories, or orbit bundles, intersect the active area of the detector. This results in a finite thickness associated with each central trajectory, which is characterized by the size of the opening of the collimator for the detector housing. . . . . . . . . . . 37 
2.4 Above is the Proton Detector 3D coordinate system. The MAST Reciprocating Probe (RP) arm lies along the x-axis, also called the r-axis. The height above the mid-plane of the tokamak is defined by the z-axis. An angle $\theta$ was chosen such that the detectors looked into the tokamak and therefore towards the plasma. The angle - $\phi$ was chosen such that the detectors looked to the left of the center column of the tokamak. The angle $\theta$, is measured from the $\mathrm{z}$-axis. The angle $\phi$ is measured from the $(-\mathrm{x}, \mathrm{z})$ plane. . . . . . . . . . . . . . . . . 40

2.5 Figure a) is a CAD side view of the mechanical arm, or reciprocating probe (RP). Figure b) is a photo of the RP. The main features of the RP are the garage, the inner tube, and the airside flange. The original CAD image of from the Culham Centre of Fusion Energy.[61][62] . . . . . . . 41

2.6 Figure a) displays a CAD image of the assembled diagnostic mechanical housing (shown without the protective BN ceramic shield). Each individual collimator opening, for each detector, is circled in red. Figure b) shows the diagnostic physically mounted to the RP. The arrow indicates the direction in which the RP pushed the diagnostic to move it closer to the plasma. The original CAD image of from the Culham Centre of Fusion Energy.[61] . . . . . . . . . . . . . . . . . 42

2.7 The entire assembled PD mechanical housing is displayed in the CAD images above; the shield is transparent in the right image. . . . . . . . . . 43

2.8 The thermal and electrical shield for the instrument was: designed, procured, cleaned, assembled (with other components into one instrument), baked, installed in an UHV environment, and then baked again before its operation for data collection. . . . . . . . . . . . . . . . . . . 44

2.9 Two detectors are housed in each module, as displayed in the CAD images above; there are a total of two modules. The modules ensure the individual $\phi$ angles for detector orientations. . . . . . . . . . . . . . . . 45

2.10 The above CAD image shows the housing which holds the detectors, or module. The module contains the: foil holder, electrical insulation for the detector, and bottom plate to hold the detectors. . . . . . . . . . . . . 46

2.11 A $0.8 \mu \mathrm{m}$ aluminum foil was spot-welded between two 316 stainless steel washers. One foil assembly was placed in front of each detector. . . . . . 47

2.12 The detector modules (displayed in the CAD images above) were physically attached to and supported by a mount and custom connector. The mount ensured the same $\theta$ orientation for all detectors. . . . . . . . . . . . 
2.13 The detector modules, mount, and custom mechanical connector were designed such that the detectors had the desired angular orientations when all of the pieces are welded together. The above left image is a CAD

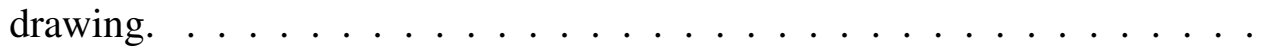

3.1 Above is a schema for the diagnostic data acquisition. RP installation constraints dictated a distance of approximately $3 \mathrm{~m}$ (blue shaded region) between the detectors and preamplifiers. The distances between items in the schema are not drawn to scale. . . . . . . . . . . . . . . . 50

3.2 The MAST RP had preinstalled UHV approved coaxial cables. A custom non-coaxial connector was installed at the end of the RP closest to the plasma. Similarly, Dsub non-coaxial connectors were required to transfer a signal out of the UHV environment and away from the vessel. An example of one detector signal being transferred through the RP shaft is displayed in the above diagram. The diagram is not drawn to scale. . . . . 51

3.3 The above images (the top image is a CAD drawing) describe the initial method used for attaching the cable screen to a pin. In the final version of the connector, instead of using a separate wire to connect the shielding to the pin, the metal braided shielding itself was unwrapped and twisted so that it was directly attached to the pin (crimp attachment). . . . . . . . . 53

3.4 The detector signals traveled through the RP airside flange and through a non-coaxial Dsub connector. Afterwards, the signal traveled to preamplifiers mounted next to the RP airside flange. The two images to the right are CAD drawings with the corresponding pin-out information for the connectors to their left. . . . . . . . . . . . . . . . . . . 54

3.5 This is an image of the first non-coaxial 15 pin Dsub female connector connected to the airside of the RP flange. . . . . . . . . . . . . . 55

3.6 This is an image of the final non-coaxial 15 pin Dsub female connector connected to the airside of the RP flange. . . . . . . . . . . 56

3.7 A copper cover was made to shield the non-coaxial connector on the outside of the RP flange (leading to the PD preamplifiers) from EMI. . . . . . 57

3.8 A box to hold preamplifiers was physically attached next to the RP airside flange. The box contains four sets of: preamplifiers, coaxial detector signal cables, preamplifier power cables, and detector bias power cables. . 58

3.9 In addition to the preamplifiers and input and output cables, braided metal shielding was attached to provide further noise reduction in the signal. . . 59 
3.10 The output signal cables from the PD preamplifiers were placed in a cable tray that led towards a cubicle containing other electronics. Slack was needed in the cables to accommodate the RP movement towards and away from the tokamak. . . . . . . . . . . . . . . . . . 59

3.11 To reduce noise caused by grounding the detector signals to the RP, the original cubicle was changed to the PD01 cubicle. The electronics: 50 $\mathrm{V}$ power supply (A), digitizer (B), CAMAC crate (C), amplifiers (D), and trigger input signal (E) were stored in the PD01 cubicle. This image shows the front of the cubicle. . . . . . . . . . . . . . . . . . 60

3.12 The electronics: $50 \mathrm{~V}$ power supply (A), digitizer (B), and CAMAC crate (C) were stored in the PD01 cubicle. This image shows the back of the PD01 cubicle. A custom power distribution unit containing fused spurs was made for the cubicle. The power for the unit led to the grounding point and power source for the RP. All electronics had the same grounding as the RP. . . . . . . . . . . . . . . . . . . . 6 61

3.13 The walls of the PD01 cubicle were electrically bonded to each other. . . . 62

3.14 The PD01 cubicle was insulated from the vessel floor through sitting on blocks of insulating material (fiber glass). . . . . . . . . . . . 63

3.15 The RP structure was grounded to the MD62 cubicle grounding point. The PD01 cubicle was grounded to the RP structure grounding point as well as to the MD62 cubicle grounding point (through the fused spurs and power distribution unit). The PD electronics (labeled 1, 2, and 3) were powered through the PD01 cubicle power distribution unit. . . . . . . . . . . 64

3.16 Custom LabVIEW code was written to remotely collect data at MAST. . . 65

4.1 Source signals due to a $5.49 \mathrm{MeV}$ alpha-particle from the decay of a 241 Am source was used to test the PD system settings to calculate expected signal sizes for protons and tritons. The caption and figure are from Perez et al., Rev. Sci. Instrum. 85, 11D701 (2014).[55] . . . . . . . . . . . . 69

4.2 These are raw data from plasma pulse 29879, Channel 2. Fusion proton signals appeared as soon as the NBI heating was applied due to the beamdominated nature of fusion reactions on MAST. The caption and figure are from Perez et al., Rev. Sci. Instrum. 85, 11D701 (2014).[55] . . . . . . 70 
4.3 These are raw data from plasma pulse 29879, Channel 1. A magnification of data signals shows the characteristic shape of a fusion proton and triton. This finite time interval is not with respect to the beginning of the pulse. The caption and figure are from Perez et al., Rev. Sci. Instrum. 85, 11D701 (2014).[55] . . . . . . . . . . . . . . 71

4.4 Above is an image of the RP position control box near the MAST control room. $A$ is the dial to set the RP position, $B$ is the button to press to move the RP towards or away from the center of the tokamak, and $C$ is the key to turn to make any changes to the RP position. Image courtesy of the

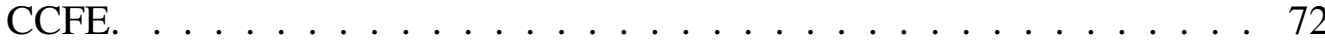

4.5 The image above displays the radial offsets of detector collimator entrances to the RP attachment point. The y-axis is vertical, r-axis is horizontal, and - z-axis goes into the page. . . . . . . . . . . . . 74

4.6 The image above displays the vertical offsets of detector collimator entrances to the RP attachment point. The z-axis is vertical, r-axis is horizontal, and $+\mathrm{y}$-axis goes into the page. . . . . . . . . . . . 74

4.7 The image above displays the horizontal offsets of detector collimator entrances to the RP attachment point. The y-axis is vertical, r-axis is horizontal, and - z-axis goes into the page. Note that the $+y$-axis is up and y-axis is down. . . . . . . . . . . . . . 75

4.8 There were periods during the data collection, circled in red in the analog data above, that indicated a possible overload or saturation of electronics. . 75

4.9 A scattering experiment with a $4 \mathrm{MeV}$ proton beam and gold target were used to test the PD electronics during varying particle rates. In this experiment, the angle between the variable detector and the beam was varied to change the observed particle rates. A control detector, or monitor, remained fixed at one angle in reference to the beam. . . . . . . . . . . 77

4.10 Data acquisition software was written in LabVIEW to collect detector voltage signals as well as display an approximate rate of particles detected $(\mathrm{Hz})$ in real time during data collection. The approximate particle rates for both the variable detector $(\mathrm{ChB})$ and the monitor detector $(\mathrm{ChA})$ were dis-

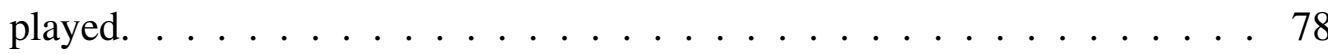

4.11 Images of an oscilloscope at various incident particle detection rates are displayed at: $6 \mathrm{kHz}, 285 \mathrm{kHz}$, and $425 \mathrm{kHz}$. The degradation of the pulse shape is greatest at $425 \mathrm{kHz}$. . . . . . . . . . . . . . . . . . 80 
4.12 The normalized experimental particle rates are graphed above. Fluctuations between the fit and experimental data indicate a lack of control in the beam direction. . . . . . . . . . . . . . . . . . . 8

5.1 Above is a schema of the data analysis code and sample plots representing the results of each of the two stages of the analysis. . . . . . . . . . 83

5.2 Characteristic proton and triton signals were observed in the raw data files. 84

5.3 Fluctuating baselines necessitate fitting the data to better determine pulse amplitudes. The red dashed line outlines the fluctuating baseline between $0.3065 \mathrm{~s}$ and $0.3075 \mathrm{~s}$ compared to a non-fluctuating baseline drawn in a dashed grey line. Data used are from Shot 29906 Channel 1. . . . . . . . . 85

5.4 High frequency signals (circled in red) necessitate fitting the data to distinguish between real events and background noise. . . . . . . . . . . 86

5.5 Above is an example of pileup occurring during high particle rates incident on the detector. The second pulse is observed on the tail of the signal of the first pulse. . . . . . . . . . . . . . . . . . . . .

5.6 Above is another example of pileup. The first pulse results when two proton pulses hit the detector within a time interval less than the pulse time resolution of the detector. The second pulse is a proton pulse with the characteristic proton height. . . . . . . . . . . . . . 88

5.7 A set of sample peaks from a channel are chosen and fit to a general pulseshape function. The analysis code then iterates through the raw data, fitting pulse-shape functions (with a quadratic background) to peaks. The Figure on the right is from Perez et al., Rev. Sci. Instrum. 85, $11 D 701$ (2014). [55] . . . . . . . . . . . . . . . .

5.8 Time intervals for sample peaks, representative of the types of pulses found throughout the channel, are selected. Figure a) displays data for an entire plasma discharge (0.12 s) while Figure b) and Figure c) display closer views of the data ( $3.5 \mathrm{~ms}$ and $15 \mu$ s time intervals). A single peak is displayed in $\mathrm{d}$ ) which spans a $1 \mu$ s time interval. . . . . . . . . . . . . 90

5.9 The individual data points (circles) for the sample peaks and the average peak shape (black triangles) are plotted along with the fit function (black line). Note that the time interval displayed is not with respect to the beginning of the plasma shot. The normalized amplitude height of each peak is

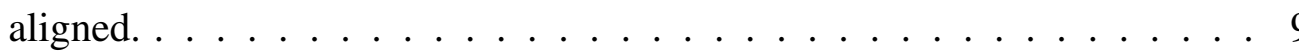


5.10 The raw data file is split into finite time intervals. The first analysis code iteratively fits data in these finite time slices (shown in gray). After the initial fitting, new finite time intervals (slices shown in red) are chosen such that they span the edges of the first set of time intervals. Finally, the central region of each fitted time interval (gray and red) is adjoined to create one file of continuously fitted data. . . . . . . . . . . . . . . . 92

5.11 The image to the left bins all of the raw (unfitted) data by signal height. Binning fitted data by pulse amplitude and using a criteria of merit ratio is displayed in the image to the right. This reduces the lower energy and noise signals contribution to the energy spectra. . . . . . . . . . . . 93

5.12 Above are histograms of pulse amplitudes for $100 \mathrm{~ms}$ time intervals using different CR values. Figure b) and e) use a CR value of 4 for Shot 29931, Channel 3. This is an appropriate value because it distinguishes between the triton signals and low energy and noise signals in a bulk of the histograms for $100 \mathrm{~ms}$ time intervals. Figure a) and d) use a CR value of 12.5, which is too high of a value because an unnecessary amount of low energy and noise signals ( $0 \mathrm{~V}$ to $0.1 \mathrm{~V}$ ) become included in the histogram. Figure c) uses a CR value of 1.1 which is too low of a value because not enough low energy and noise signals are histogrammed. . . . . . . . . . 95

5.13 Proton and triton energy spectra are plotted above. The proton peaks are in the red box to the right. The red box ranges from the minimum to maximum proton peak values. The triton peaks are in the black box to the left. The arrows point to the mean values of the triton and proton peaks. For this example the control file parameter values used are: $p_{\min }=0.5 \mathrm{~V}$, $p_{\text {max }}=0.7 \mathrm{~V}, p_{\text {mean }}=0.6 \mathrm{~V}, T_{\text {min }}=0.13 \mathrm{~V}, T_{\text {max }}=0.31 \mathrm{~V}$, and $T_{\text {mean }}=0.21 \mathrm{~V} . .96$

5.14 The above image is qualitative. Integrating signals in the proton energy spectra yields the total number of protons detected in a finite time interval, $\Delta \mathrm{t}$. The ratio of the proton sum and $\Delta \mathrm{t}$ corresponding to the histogram is plotted as a rate value. A collection of these data points (from a collection of histograms) creates an emission rate of protons during a plasma discharge. 99

5.15 A proton production rate plot, where each point represents a sum taken over successive histograms of $1 \mathrm{~ms}$ time intervals. . . . . . . . . . . . 100

5.16 The above image is qualitative. The above figure displays the characteristic mid-plane radii (the $\mathrm{R}$ value when $\mathrm{Z}=0$ ) for the four $\mathrm{PD}$ channels as well as the magnetic axis. . . . . . . . . . . . . . . . . 102 
6.1 Above is a general image for Rutherford scattering where $\mathrm{b}$ is the distance of closest approach of $m$ to M.[66] Note that if $m$ is the electron mass and $\mathrm{M}$ is an ion of positive charge, then $\mathrm{m}$ would deflect towards the ion. . . . 103

6.2 During several MAST discharges, two successive neutral beams were injected into the plasma. The regions of exponential decay after NBIs end are highlighted in gray. Note that the first NBI power reaches approximately 1.5 MW and the second NBI power reaches approximately 1 MW. . 111

6.3 Above are the experimental proton and neutron time decay constants, $\tau$ (tau), for two different time intervals. Data from the fission chamber (FC) are used for the neutron time decay constants. . . . . . . . . . 113

6.4 The experimental proton time decay constants as a function of experimental neutron time decay constants. The legend for the plot is in a box which is shaded light blue. . . . . . . . . . . . . . . . . . . . . . 115

6.5 The experimental proton and neutron time decay constants, $\tau_{1}$ and $\tau_{2}$ as a function of mid-plane radii. the radial value for the magnetic axis is plotted as the solid black vertical line in each figure. The legend for each plot is in a box which is shaded light blue. . . . . . . . . . . 116

6.6 Above is a qualitative image displaying an example fishbone event observed in Mirnov coils. . . . . . . . . . . . . . . . . . . . . . . 117

6.7 Above are data for plasma pulse 29909 from the Proton Detector (all channels), Fission Chamber, Mirnov Coil, and NBI power. The red vertical line indicates an event of interest to be discussed. The quiescent period, 235 $\mathrm{ms}$ to $260 \mathrm{~ms}$, is shaded in red. . . . . . . . . . . . . 120

6.8 Above are PD channel 1 rates with the quiescent period, $235 \mathrm{~ms}$ to 260 $\mathrm{ms}$, is shaded in red. . . . . . . . . . . . . . . . . 122

6.9 Above is the orbit output for the poloidal trajectories for each PD channel. The mid-plane radii for each channel is labeled above.The mid-plane radii for channels $0,1,2$, and 3 are approximately $98.5+/-3 \mathrm{~cm}, 81.5+/-3 \mathrm{~cm}$, $86.8+/-3 \mathrm{~cm}$, and $92+/-3 \mathrm{~cm}$, respectively. . . . . . . . . 122

6.10 Above are data for plasma pulse 29909 from the Proton Detector, Fission Chamber, and Mirnov Coil. Rates from a time interval during the quiescent period are labeled as $Q u$. There is a gap in channel 1 rates (around $305 \mathrm{~ms}$ ) because pulses were not able to be resolved from noise signals in the raw data during that specific time interval. The red vertical line indicates an event of interest to be discussed. . . . . . . . . . . . . . . 123 
6.11 Above are data for plasma pulse 29931 from the Proton Detector, Fission Chamber, Mirnov Coil, and NBI power. The red vertical line indicates an event of interest to be discussed. . . . . . . . . . . . . . . . . . . 125

6.12 Above are data for plasma pulse 29931 from the Proton Detector, Fission Chamber, Mirnov Coil, and NBI power. The red vertical line indicates an event of interest to be discussed. . . . . . . . . . . . . . . . . . . . 126

6.13 Above is the orbit output for the poloidal trajectories for each PD channel. The mid-plane radii for each channel is labeled above. The mid-plane radii for channels 1,2 , and 3 are approximately $82.1+/-3 \mathrm{~cm}, 87.4+/-3 \mathrm{~cm}$, and $92.5+/-3 \mathrm{~cm}$, respectively. The first mid-plane radii for channel 0 is approximately $100.4+/-3 \mathrm{~cm}$. . . . . . . . . . . . . 126

6.14 Plotting the falls in DD proton and global neutron rates as a function of mid-plane radii indicates that the largest decrease in fast ion rates occur closest to the plasma core. The black vertical line indicates the radial value of the magnetic axis. For shot 29909, each PD data point from left to right represents: Ch0, Ch1, Ch2, and Ch3. For shot 29931, each PD data point from left to right represents: $\mathrm{Ch} 1, \mathrm{Ch} 2$, and $\mathrm{Ch} 3 . \ldots . . .127$

6.15 Above is a qualitative image displaying sample sawteeth events. The ramp up phase and crash, or collapse, phase are labeled. . . . . . . . . . . . . 128

6.16 Above is a time interval of the plasma electron density during plasma pulse 29879 in which successive sawtooth events can be observed. . . . . 129

6.17 Above are data for sawtooth crashes in plasma pulse 29879 from the PD and fission chamber. Data used are from Perez et al., Rev. Sci. Instrum. 85, 11D701 (2014).[55] . . . . . . . . . . . . . . . . 130

6.18 Above are data from the Proton Detector and the MAST Neutron Camera during plasma pulse 29879. The units of labeled mid-plane radii are $\mathrm{m}$. The horizontal vertical line indicates the sawtooth crash of interest that is discussed. Caption and data used in Figure are from Perez et al., Rev. Sci. Instrum. 85, 11D701 (2014).[55] . . . . . . . . . . . . . . . 131

6.19 Above is a graph of global DD proton and neutron production in the plasma from TRANSP simulation output for a plasma pulse containing sawtooth events (with similar plasma properties as pulse 29879). TRANSP is a time dependent energy transport analysis code developed by the PPPL.[60] 132

6.20 Above is the radial emission profile for before and after the second crash in pulse 29879. A modulated power law is used to fit the proton emissivity. The radial value of the magnetic axis is $0.94 \mathrm{~m} . \ldots 135$ 
6.21 The fitted emissivity profile, before and after the second crash, using a modulated power law function for pulse 29879 appear above. The emissivity profile is plotted against the relative magnetic flux surfaces of the

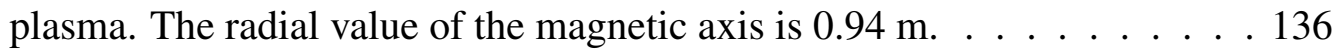

6.22 Above is the fitted emissivity, based on a modulated power law, for each channel versus the relative magnetic flux. . . . . . . . . . . . 137

6.23 Above is the radial emission profile for before and after the second crash in pulse 29879. A modulated power law is used to fit the proton emissivity. The radial value of the magnetic axis is $0.94 \mathrm{~m}$. Figure and Caption are from Perez et al., Rev. Sci. Instrum. 85, 11D701 (2014).[55] . . . . . . 139

6.24 The fitted emissivity profile, before and after the second crash, using a Gaussian function for pulse 29879 appear above. The emissivity profile is plotted against the relative magnetic flux surfaces of the plasma. The radial value of the magnetic axis is $0.94 \mathrm{~m}$. Figure and Caption are from Perez et al., Rev. Sci. Instrum. 85, 11D701 (2014).[55] . . . . . . . . . 140

6.25 Above is the fitted emissivity, based on a Gaussian function, for each channel versus the relative magnetic flux. Figure and caption from Perez et al., Rev. Sci. Instrum. 85, 11D701 (2014).[55] . . . . . . . . . . . . 141

7.1 Above is an image of two sets of detector array poloidal trajectories projected onto an orbit output simulation. This is a possibility for future PD diagnostics which have more than one probe head inserted into the tokamak.145 


\section{CHAPTER 1}

\section{INTRODUCTION AND MOTIVATION}

The following introduction and background provides the motivation for my dissertation work within the context of experimental plasma physics and fusion research.

\subsection{Plasma Properties in Fusion Research}

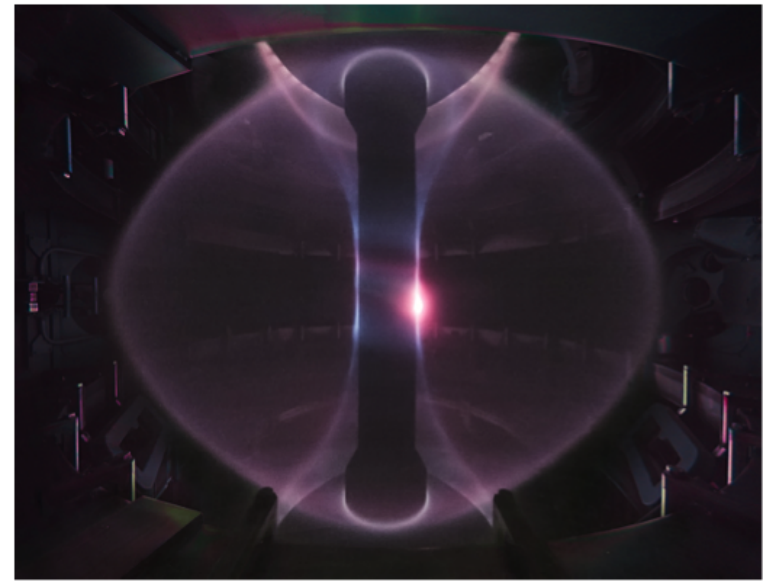

Figure 1.1: A color composite image of a typical plasma in the Mega Amp Spherical Tokamak (MAST) fusion device at the Culham Centre for Fusion Energy.[31]

Plasmas, ionized gaseous mixtures, are used for fusion research. They can reach temperatures of over one hundred and fifty million degrees Celsius $\left({ }^{\circ} \mathrm{C}\right)$, or fifteen kiloelectronvolts $(\mathrm{keV})$, which is ten times hotter than the center of the sun. Their densities can be greater than those found in the center of the $\operatorname{sun}^{1}$.[9] With a high fraction of ionization and free electrons, collective effects of plasmas are dominated by electromagnetic interactions

\footnotetext{
${ }^{1}$ These high density plasmas can be found at the National Ignition Facility at Lawrence Livermore National Laboratory in Livermore, California. These plasmas last for only a few nanoseconds.
} 
and they have high electrical and thermal conductivity. Plasmas used in fusion research are fully ionized.[4] The densities of these electrons and ions within the plasma can give rise to large currents, forces, electric fields, and magnetic fields.

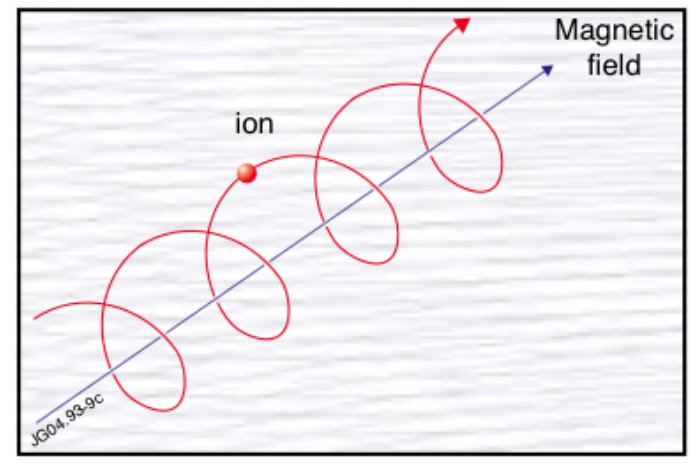

Figure 1.2: Charged particles moving along magnetic field lines will rotate, or gyrate, in a direction perpendicular to the magnetic field. The resulting path, or trajectory, of the particle is helical. The mass and charge of the particle will determine the direction and the size of gyration.[40]

Ions and electrons in a magnetic field follow helical trajectories centered on magnetic field lines, as determined by the Lorentz force law (1.1). These trajectories, in which charged particles rotate or gyrate, are displayed in Figure 1.2. In general, the equation of motion of a moving charged particle in a magnetic field can be described by ${ }^{1}$,

$$
m \frac{d \vec{v}}{d t}=q \vec{v} \times \vec{B}
$$

where $\mathrm{m}$ is the particle mass, $\mathrm{q}$ is its charge, $\vec{v}$ its velocity, and $\vec{B}$ is the external uniform magnetic field. With a magnetic field in the $\mathrm{z}$ direction the particle's circular motion in the plane perpendicular to the magnetic field (x-y plane) moves with a frequency $\omega$, also called the gyro-frequency.

$$
\omega=\frac{q B}{m}
$$

\footnotetext{
${ }^{1}$ Note that the particles of interest for which this is relevant have energies ranging from $0.8 \mathrm{MeV}$ to $3 \mathrm{MeV}$. Therefore their Lorentz factors, $\gamma=\left(1-\left(v^{2} / c^{2}\right)\right)^{-1 / 2}$, are close to 1 making the use of non-relativistic equations of motion appropriate. The $\gamma$ for protons, tritons, and helium-3 ions are approximately 1.003, 1.001, and 1.003, respectively.
} 
This circular motion, or gyro-motion, has a corresponding gyro-radius. Therefore looking at the force acting on the particle in a direction perpendicular to the magnetic field from (1.1) and using the centripetal force for circular motion

$$
\frac{m v_{\perp}^{2}}{r}=|q| v_{\perp} B
$$

yields

$$
r=\frac{m v_{\perp}}{|q| B}
$$

where the gyro-radius (1.4) is measured from the guiding center. The center of the gyromotion is the guiding center which moves approximately along a field line. The total motion of the particle consists of its gyro-motion about the guiding center and the guiding center motion.

Additionally, particles can experience drift velocities caused by gradients in the magnetic field or forces perpendicular to the magnetic field.[58] In the presence of a uniform force in addition to a magnetic field, where this extra force $\vec{F}$ would be added to (1.1), a particle will experience a drift velocity in addition to the gyro-motion. Drift velocity can be expressed as (1.5).

$$
\vec{v}_{d r i f t}=\frac{(\vec{F} \times \vec{B})}{q B^{2}}
$$

When the electric force, $\vec{F}=q \vec{E}$, for a uniform electric field is added to (1.1), the total force in the equation of motion is called the Lorentz force.

$$
\vec{F}_{\text {Lorentz }}=q(\vec{E}+\vec{v} \times \vec{B})
$$

As particles travel throughout a plasma, trapping them at a high enough energy, or temperature, for a sufficient time period such that they undergo nuclear fusion reactions requires magnetic confinement. A closed system will minimize the loss of particles. 
Therefore, creating a torus in which helical magnetic field lines close on themselves minimizes the loss of particles traveling along those magnetic field lines. Large machines called tokamaks use the method of magnetic confinement to create plasmas for fusion research. Existing research facilities not using magnetic confinement lie outside the scope of this work.

Nuclear Fusion Power Reactors Current nuclear power plants harvest energy from nuclear fission reactions. A thermal neutron and a Uranium nucleus undergo a fission reaction (1.7) resulting in $84 \times 10^{6}$ MegaJoules (MJ) of energy per kilogram (kg) of reactant.[3]

$$
U^{235}+N=C e^{140}+Z r^{94}+2 N+6 e^{-}
$$

The concept for fusion power reactors, instead, harvests energy from nuclear fusion reactions between hydrogen isotopes; see the DT reactivity in Figure 1.3. The fusion of deuterium and tritium (1.8) can result in $338 \times 10^{6} \mathrm{MJ}$ of energy per kg of reactant which is four times as much energy release as a nuclear fission reaction.[3]

$$
D+T=N(14.1 \mathrm{MeV})+H e^{4}(3.5 \mathrm{MeV})
$$

In a fusion reactor, energetic neutrons leaving the reactor would heat up the vessel wall and surrounding materials. The heat energy eventually turns water into steam which can then power steam turbines. Prominent themes in fusion research and experimental plasma physics are in support of the future reactor design which makes use of plasmas. A detailed discussion of fusion power reactor designs is outside the scope of this work; this brief section serves to provide a context for the motivation of this dissertation work as it relates to the fusion research community. 


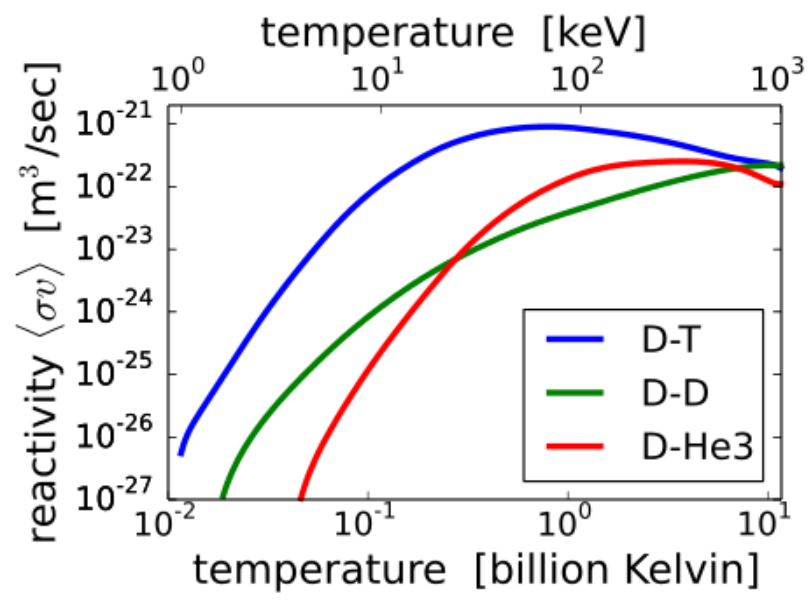

Figure 1.3: The reactivity (proportional to the experimental cross section) for several nuclear fusion reactions are displayed above. The relationship between temperature and the probability of nuclear fusion reactions occurring provides the motivation for fusion plasma operating temperatures.[33]

\subsection{Producing Plasmas for Fusion Research}

\begin{tabular}{|c|c|c|c|}
\hline Machine & Major Radius [m] & Minor Radius [m] & Aspect Ratio \\
\hline MAST & 0.85 & 0.65 & 1.3 \\
\hline NSTX & 0.85 & 0.65 & 1.3 \\
\hline JET & 2.96 & 1.25 & 2.37 \\
\hline ITER & 6.2 & 2.0 & 3.1 \\
\hline
\end{tabular}

Table 1.1: This table contains several aspect ratios for several tokamaks.[1][3][6][36]

The two main types of tokamaks are the conventional and spherical tokamak. Conventional tokamaks create a toroidal-shaped plasma while spherical tokamaks (STs) create a toroidal-shaped plasma that has a cross-section resembling a coreless apple. Figure 1.4 displays the difference between the major and minor radii of spherical tokamaks (drawn in red) and conventional tokamaks (drawn in black). The minor radius, $r_{m}$, is measured from the center of the plasma's poloidal cross-section to the plasma edge. The major radius, $R_{m}$, is measured from the center of the plasma to the center of the poloidal cross-section. The 
aspect ratio $A_{m}=R_{m} / r_{m}$ is a common parameter used to compare tokamaks of different sizes, see Table 1.1. For example, the International Thermonuclear Experimental Reactor (ITER) tokamak is planning a $6.2 \mathrm{~m}$ major radius and $2 \mathrm{~m}$ minor radius, meaning a 3.1 aspect ratio and $837 \mathrm{~m}^{3}$ plasma volume.[3] The Joint European Torus (JET) has a 2.37 aspect ratio.[36] The National Spherical Torus Experiment (NSTX) and Mega Amp Spherical Tokamak (MAST) have the same 1.3 aspect ratio with approximate plasma volumes of $8 \mathrm{~m}^{3}$.[6][1] Plasmas created at NSTX and MAST are typically composed of deuterium (hydrogen isotopes), see Figure $1.1^{1}$.

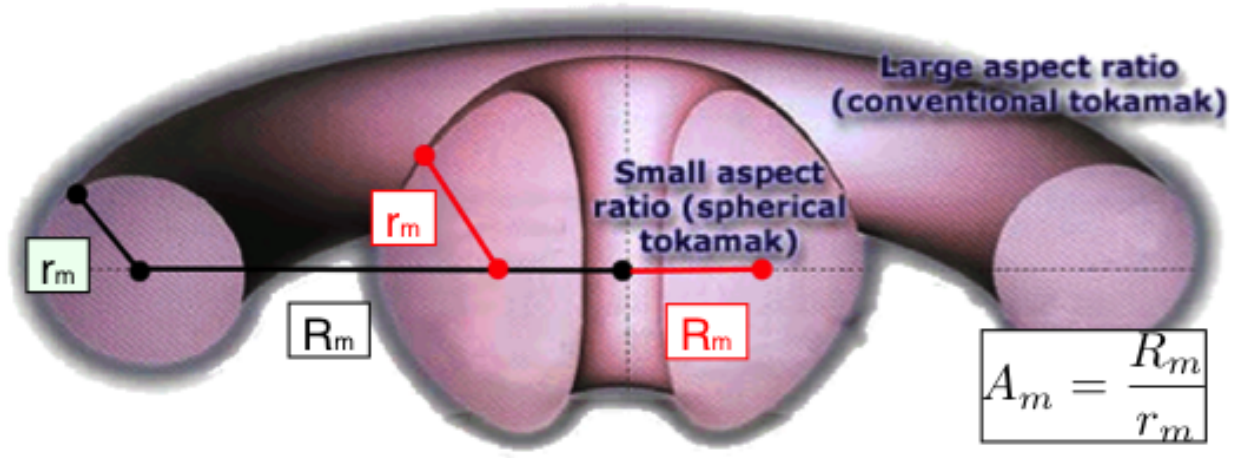

Figure 1.4: The above image is qualitative. Different tokamaks have different aspect ratios, or ratios of their major radii to minor radii. The aspect ratio can be calculated using the expression $A_{m}=R_{m} / r_{m}$. The original figure is from the Culham Centre of Fusion Energy.[10].

These hot plasmas are created in pulse-mode operation, meaning each time they are created they can last from less than one second to several minutes before dissipating. For example, JET can create plasma pulses lasting several minutes while NSTX can create pulses lasting a few seconds.[36][6] Typical MAST pulses last between $0.5 \mathrm{~s}$ and $1 \mathrm{~s}$. ITER is planning a $400 \mathrm{~s}$ plasma pulse length.[3]

\footnotetext{
${ }^{1}$ Figure 1.1 is a composite image of a deuterium plasma. The visible sheath of the plasma is the coldest region where deuterium atoms are not fully ionized and are still exchanging electrons; this process emits a pink or purple light. The hottest central region of the plasma is fully ionized and does not emit light in the visible spectrum.
} 


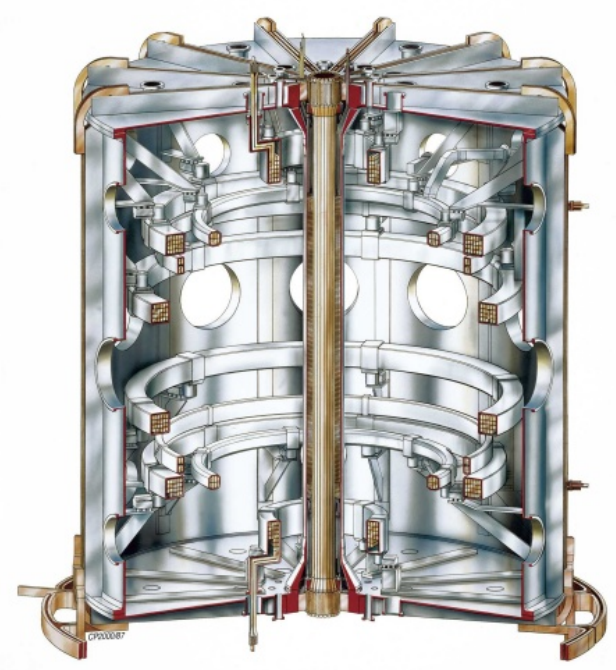

Figure 1.5: A cross-sectional view (CAD drawing) showing the Mega Amp Spherical Tokamak (MAST) at the Culham Centre for Fusion Energy in Abingdon, the United Kingdom.[35]

Tokamak Startup and Ohmic Heating A high vacuum (HV) of the order $10^{-8}$ Torr is created inside of the tokamak vessel. Then when a puff of hydrogen gas (such as deuterium) is injected into the tokamak vessel, a current is run through a set of coils in the center of the vessel. The induced current heats up and ionizes the gas, creating a plasma. The process of inducing a current in the plasma is analogous to the way a transformer induces current. The tokamak acts like a transformer where the central solenoid is the primary coil and the plasma is the secondary coil. The process of heating the plasma through an induced current is called ohmic heating or resistive heating (see Figure 1.6). Additionally, this process of rapidly ramping up the current in these coils induces a toroidal electric field.

As the plasma temperature increases, however, the plasma becomes highly conductive and the resistivity decreases. Therefore as the plasma temperature approaches a few $\mathrm{keV}$, 


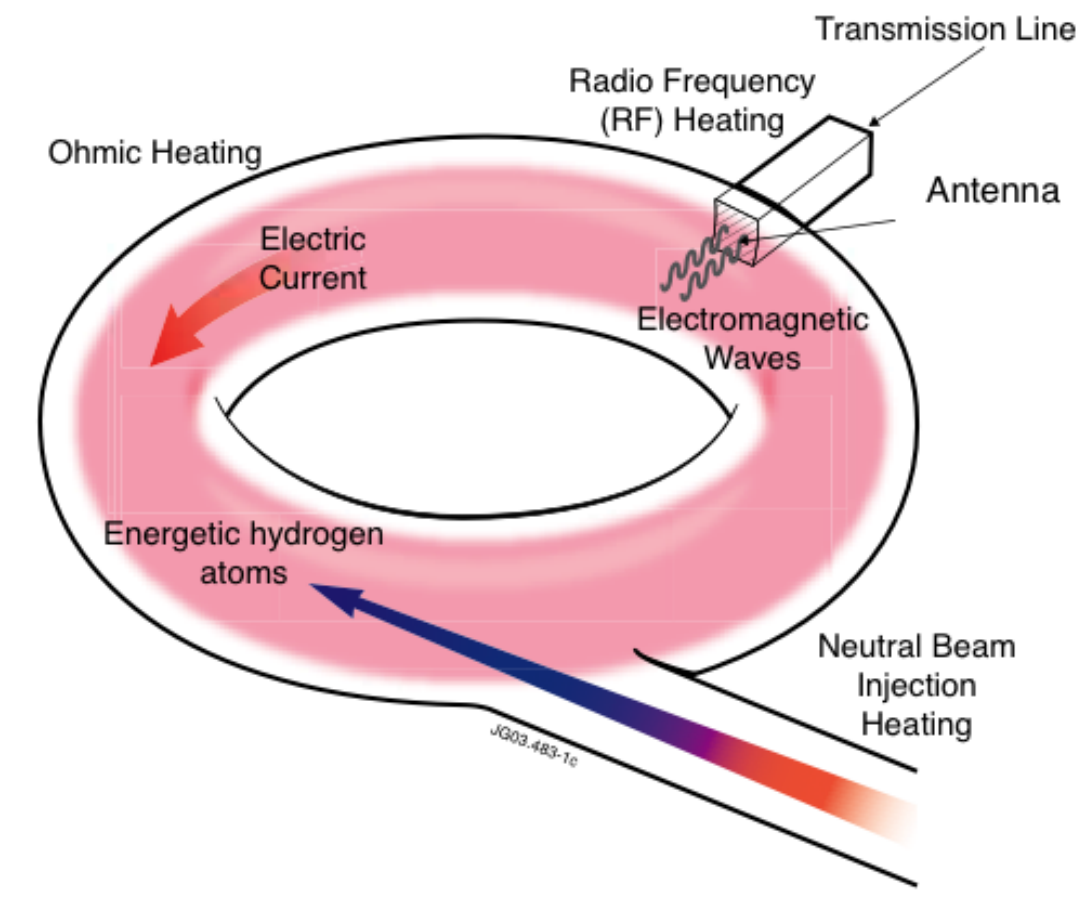

Figure 1.6: Three main methods are used to heat a plasma to operating temperature, a temperature high enough to sustain deuterium-deuterium nuclear fusion reactions. The first method is ohmic heating, which is followed by a combination of radio frequency heating and the injection of an energetic neutral beam. [38].

ohmic heating becomes less effective at higher temperatures. Subsequently, to increase the plasma temperature from a few $\mathrm{keV}$ to $15 \mathrm{keV}$, two additional heating methods are employed: radio frequency heating and neutral beam injection.[3] ${ }^{1}$

Magnetic Confinement in Tokamaks Tokamaks use the method of magnetic confinement to shape and contain plasmas. The Mega Amp Spherical Tokamak (MAST) has a toroidal magnetic field of approximately $0.6 \mathrm{~T}$ while ITER is planning a $5.3 \mathrm{~T}$ field.[3] To create the toroidal component of the magnetic field, current is run through a set of vertical coils $^{2}$. The second component of the magnetic field is created when current runs through

\footnotetext{
${ }^{1}$ Note that during this process, small pellets of deuterium (frozen at $-269^{\circ} \mathrm{C}$ ) are shot into the plasma at a speed of 350 to 500 meters per second. The pellets evaporate and ionize when they enter the plasma thereby raising the plasma density; up to eight pellets can be used in one plasma discharge during a spherical tokamak's pulse-mode operation.[2]

${ }^{2}$ Running a current through a coil creates a uniform magnetic field at the center of the coil.
} 


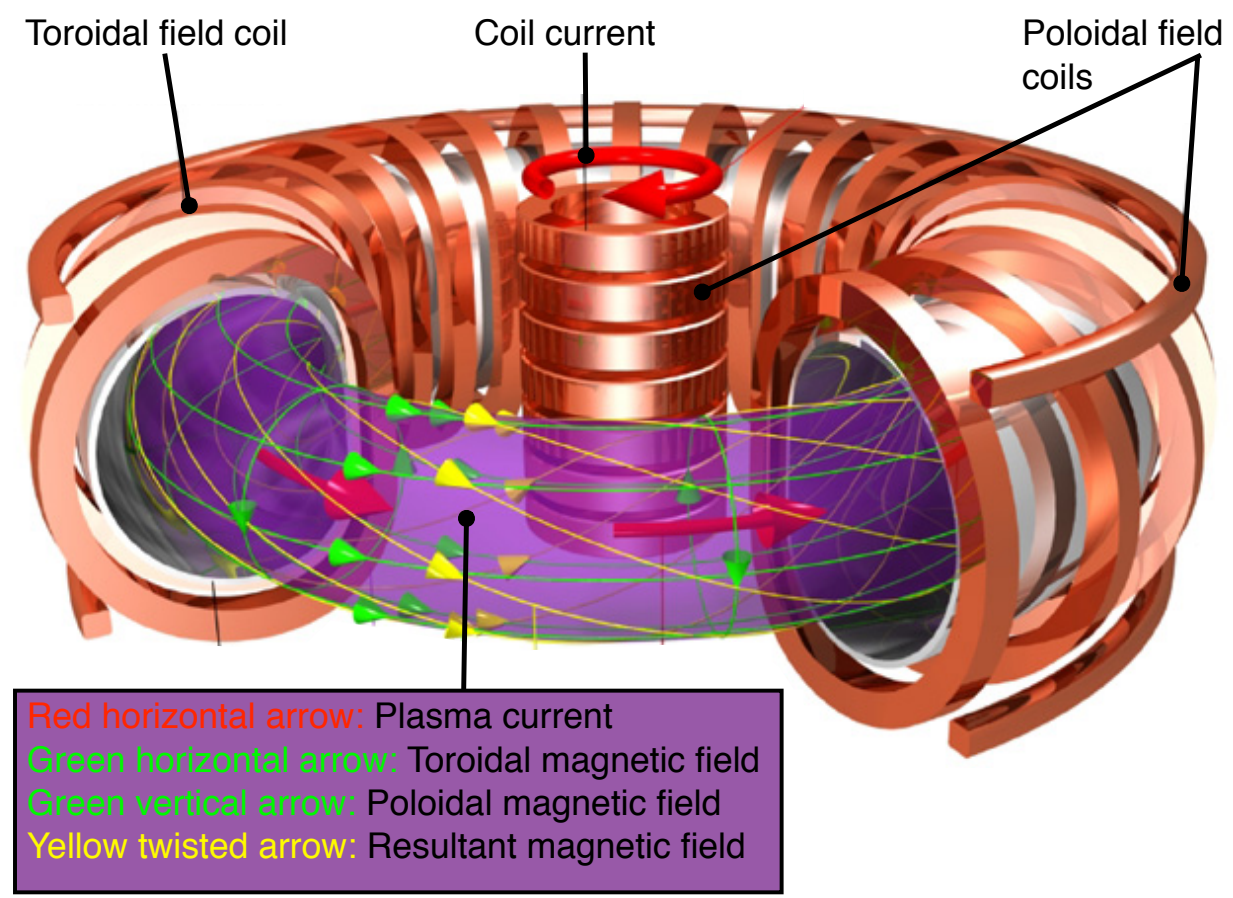

Figure 1.7: A tokamak uses horizontal, or toroidal, magnetic field lines to confine a plasma in the horizontal direction. It also uses vertical, or poloidal, magnetic field lines to confine a plasma in the vertical direction. Together, the resultant magnetic field lines that confine the plasma are twisted, or helical.[32]

the outer horizontal coils to create a poloidal magnetic field. Additionally, the previously discussed induced current in the plasma will create a poloidal magnetic field. Values for these parameters can be found in Table 1.2. Both components of the magnetic field will combine to produce a helical-shaped magnetic field as seen in Figure 1.7. Note that the resultant magnetic field prevents the plasma from physically touching the walls of the tokamak vessel. Again, these resultant magnetic field lines closing on themselves in a torus

\begin{tabular}{|c|c|c|}
\hline Machine & Toroidal $B_{T}[\mathrm{~T}]$ & $\mathrm{I}[\mathrm{MA}]$ \\
\hline MAST & 0.6 & 1.3 \\
\hline NSTX & 0.55 & 1.5 \\
\hline JET & 3.45 & 4.8 \\
\hline ITER & 5.3 & 15 \\
\hline
\end{tabular}

Table 1.2: This table contains characteristic values for the toroidal magnetic field and current used for different tokamaks to confine plasmas.[36][6][1][3] 
creates a closed system which minimizes particle loss, and thus energy, from the plasma. Though this dissertation work focuses on studying plasmas created by a ST, the same concept of magnetic confinement is applicable to both STs and conventional tokamaks. A cross-sectional view of the coils used in MAST is shown in Figure 1.5.

Radio Frequency Heating Figure 1.6 displays another method used to heat the plasma after ohmic heating: radio frequency $(\mathrm{RF})$ heating. In general, a high voltage power supply is used to power an RF source which then transfers the wave along a transmission line to an antenna.[58] When these electromagnetic waves propagate into the plasma and are in resonance with the natural frequencies of electrons and ions in the plasma, power is absorbed in the plasma.[3] The process is capable of adding up to several megawatts (MW) of power into an ST plasma.[3]

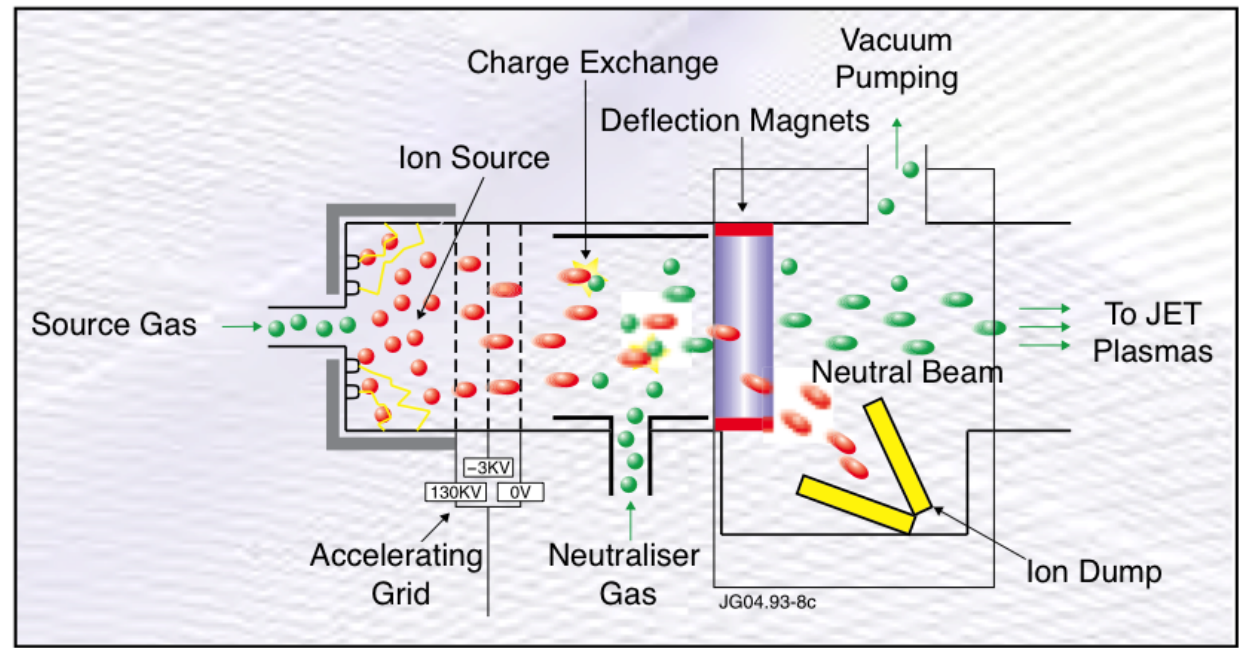

Figure 1.8: This diagrams shows the basic process of creating a beam of energetic neutral atoms for the Joint European Torus (JET).[39]

Neutral Beam Injection (NBI) An energetic beam of neutral deuterium atoms is created through accelerating ionized deuterium gas by a large potential difference, for example of the order of $100 \mathrm{kV}$ for JET and $50 \mathrm{kV}$ to $60 \mathrm{kV}$ for MAST. After applying the 
acceleration voltage, these collimated energetic ions pass through a neutralizer gas (see Figure 1.8). These fast ions undergo inelastic collisions, or charge exchange collisions, with the neutral atoms of the neutralizer gas. For example, if positive ions pass through the neutralizer, the high-energy positive ions become high energy neutral atoms while the low energy neutral atoms become low energy ions.[3] Three types of particles leave the neutralizer: high energy neutral atoms, low energy ions, and high energy ions which do not undergo charge exchange collisions ${ }^{1}$. These particles then pass through an external magnetic field created by a magnet. The magnetic field produces a force acting on any ions through curving their trajectory (1.4), deflecting them towards what is called a beam dump. The beam dump collects the ions before they enter the plasma. While the ions are deflected, the magnetic field does not affect the trajectory of the high energy neutral atoms, which continue towards the plasma.[3]

The beam of neutral deuterium atoms, with an approximate energy of $50 \mathrm{keV}$ to $60 \mathrm{keV}$ for MAST, travels into the central region of the plasma (see method three in Figure 1.6). These ions must be neutralized so they can penetrate the plasma's magnetic field. When the beam atoms are injected into the plasma, they ionize and transfer momentum to plasma electrons and ions through Coulomb collisions. The process heats the plasma. Chapter 6 will discuss these collisions in more detail. The Mega Amp Spherical Tokamak (MAST) delivers up to $5 \mathrm{MW}$ of neutral beam power while ITER is planning to deliver $33 \mathrm{MW}$ of neutral beam power, see Table 1.3 for more values.[3][1]

Equations (1.9), and (1.10) indicate the primary DD nuclear fusion reactions taking place in the plasma at the time of the neutral beam injection (NBI), see Figure 1.9. Approximately $85 \%$ of these reactions occur between energetic beam ions and the plasma ions, beam-target (BT) reactions, while only approximately $10 \%$ of these reactions occur between the energetic beam ions themselves, beam-beam BB reactions.[12] The amount of

\footnotetext{
${ }^{1} \mathrm{~A}$ neutralizer is not $100 \%$ efficient.
} 


\begin{tabular}{|c|c|}
\hline Machine & NBI Heating Power [MW] \\
\hline MAST & 5 \\
\hline NSTX & 7 \\
\hline JET & 23 \\
\hline ITER & 33 \\
\hline
\end{tabular}

Table 1.3: This table contains characteristic values for the neutral beam heating power for several tokamaks.[36][6][1][3]
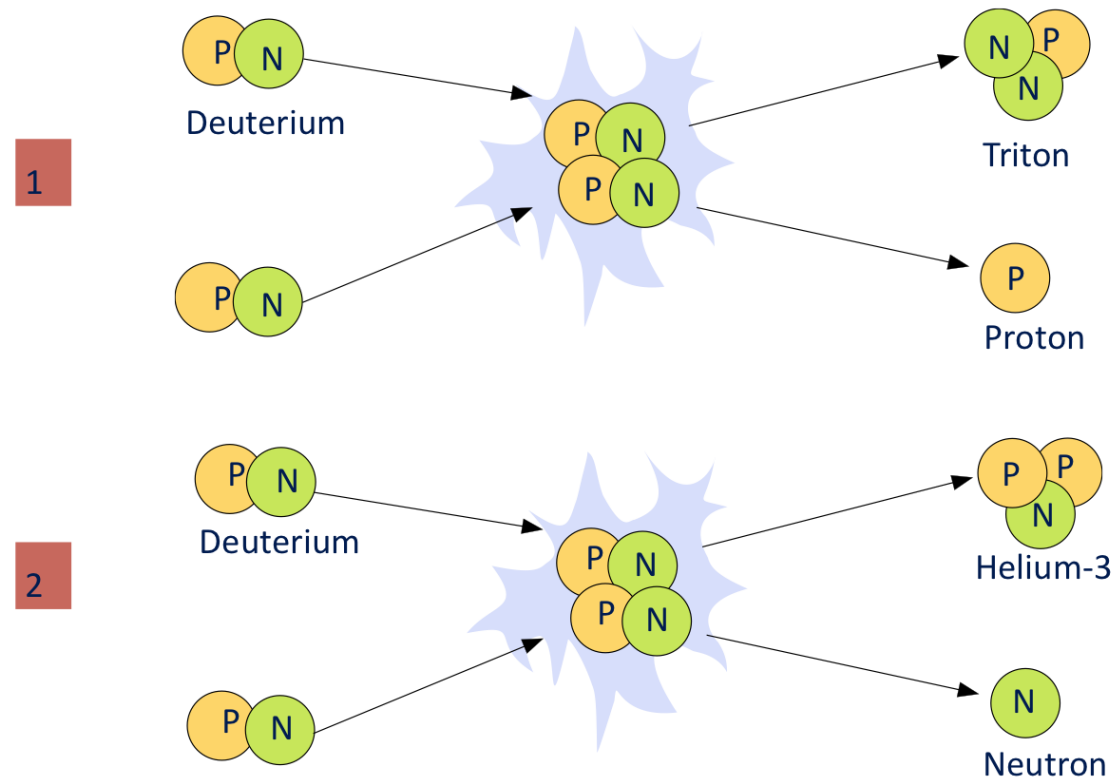

Figure 1.9: During a nuclear fusion reaction inside of a plasma, when two deuterium atoms fuse, they can create either a proton $(\mathrm{P})$ and a triton or a neutron (N) and a helium-3 ion.

BT reactions means that the largest contribution of fusion products takes place during the neutral beam injection. Both branches of the DD fusion reactions are shown in (1.9) and (1.10).

$$
\begin{gathered}
D+D=P(3.02 M e V)+T(1.01 M e V) \\
D+D=N(2.45 M e V)+H e^{3}(0.82 M e V)
\end{gathered}
$$


The plasma's toroidal current is crucial to maintain plasma confinement. Therefore noninductively maintaining the current drive for longer periods of time to achieve a steady-state mode of operation remains a major focus within fusion research.[3]

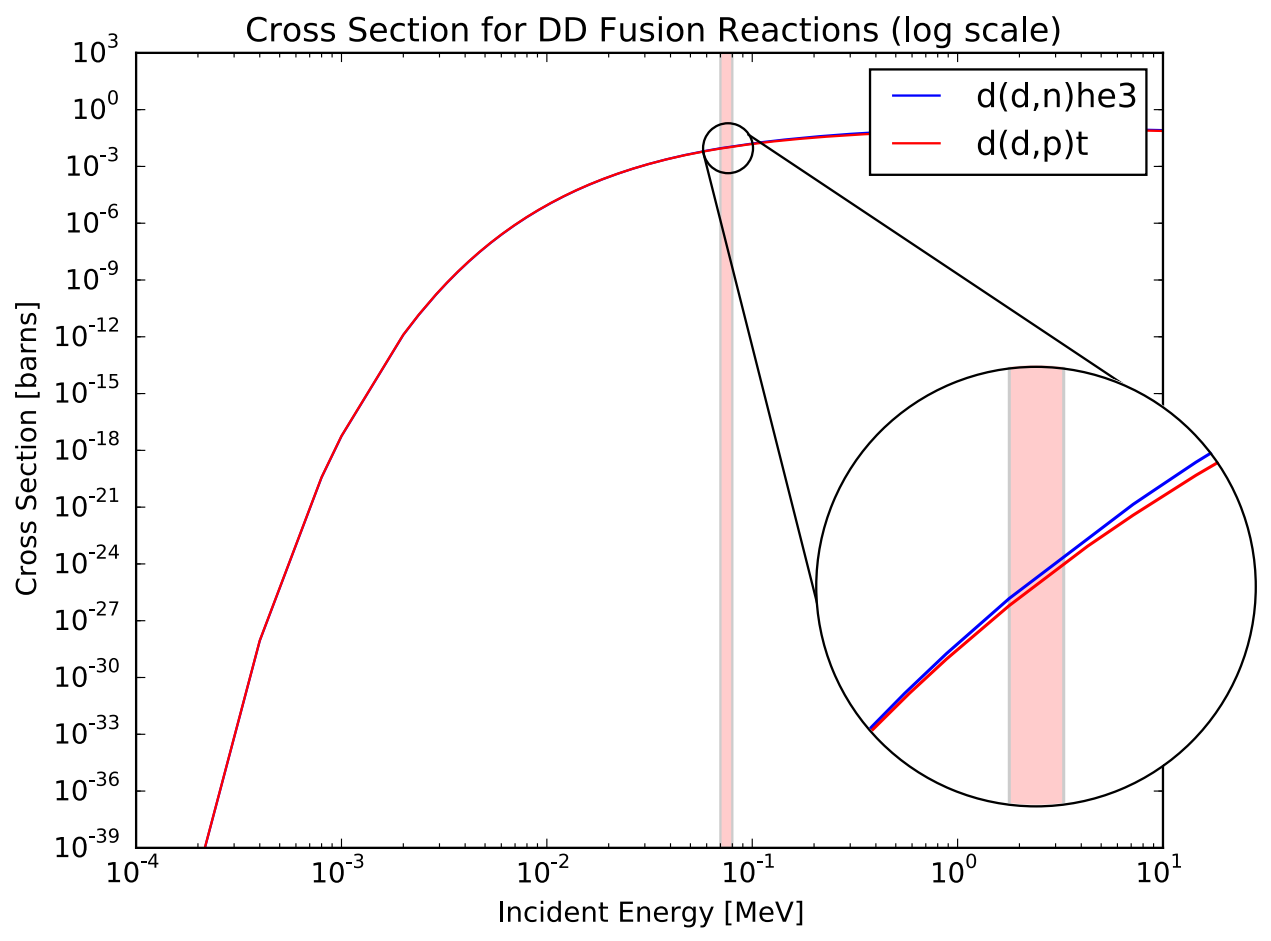

Figure 1.10: Both branches of a DD fusion reaction, (1.9) and (1.10), have approximately the same cross section. Therefore, they each have approximately the same probability of occurring $(\sim 50 \%)$. At $70 \mathrm{keV}$ the experimental cross section for the $\mathrm{d}(\mathrm{d}, \mathrm{n})$ he3 and $\mathrm{d}(\mathrm{d}, \mathrm{p}) \mathrm{t}$ branch are 0.0091 barns (unit of area) and 0.0088 barns, respectively. Data used to make the graph are from the Evaluated Nuclear Data File (ENDF) Database.[11]

Branches of DD Fusion Reactions The probability of neutrons or protons being produced, (1.9) and (1.10), are approximately 50\%. See Figure 1.10 for the experimental cross sections of the two possible sets, or branches, of DD fusion reaction products. Though the probability for each branch is approximately the same, the reactions are not isotropic meaning when a DD reaction occurs the products are not emitted randomly in space. The 
differential cross section as a function of scattering angle for both branches shows anisotropy, see Figure 1.11. In general while the anisotropy is larger for higher deuteron energies, the anisotropy is larger for the set of neutron and helium-3 ion products.[8]

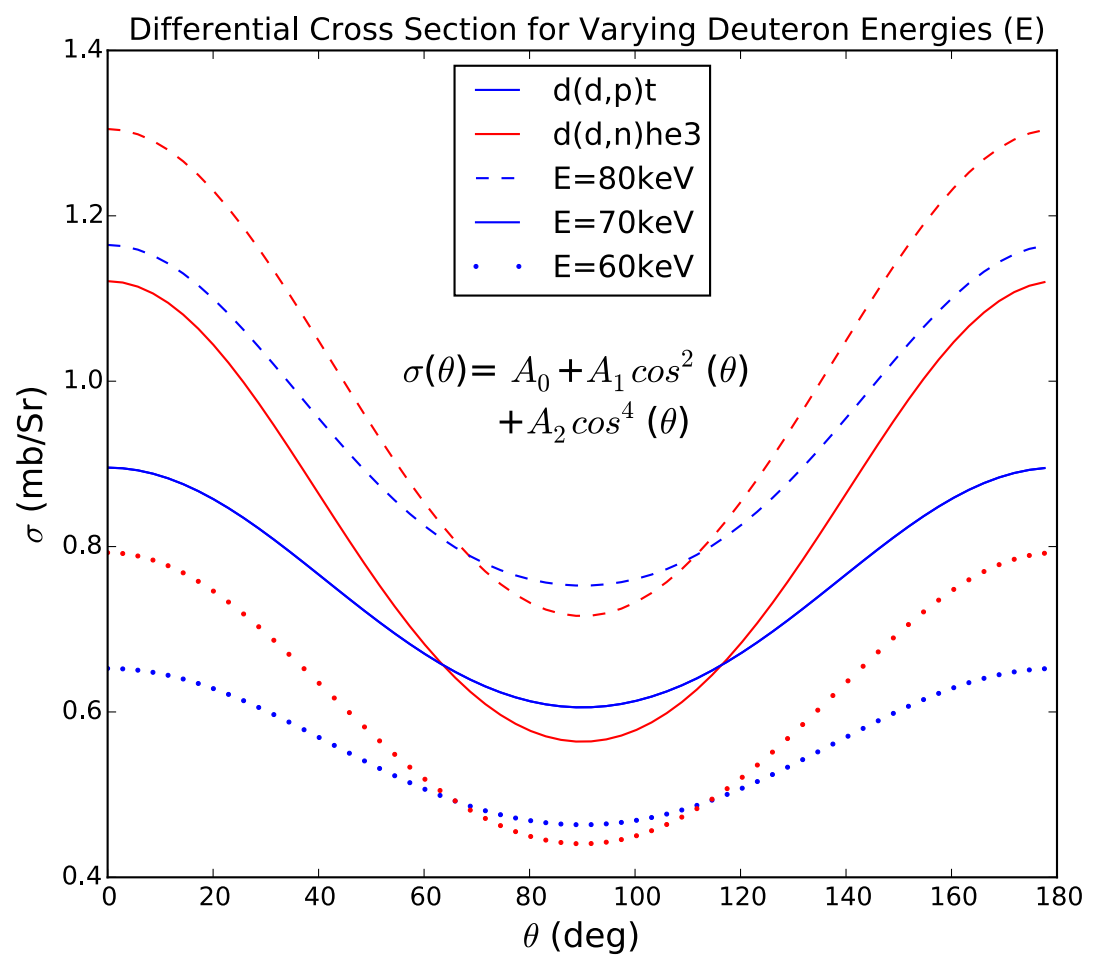

Figure 1.11: The differential cross section for both DD fusion reaction branches shows anisotropy. The anisotropy can vary in range depending on the reaction branch and the initial deuteron energy (E). The constants $A_{0}, A_{1}$, and $A_{2}$ change depending on $\mathrm{E}$ and the reaction branch. The units for $\sigma$ are millibarns (mb) per steradian ( $\mathrm{sr}$ ). Data and the form of $\sigma(\theta)$ used to make the graph are from the Lawrence Livermore National Laboratory.[8]

Tokamak Diagnostics Several dedicated instruments, or diagnostics, are permanently installed around a tokamak's vessel to measure relevant plasma characteristics. These parameters include the electron temperature, electron density, toroidal plasma current, toroidal magnetic field strength, and oscillations in the magnetic field. For example, MAST takes measurements of these parameters every time it creates a plasma. Recording these data 
throughout a plasma discharge is not only essential to support studies of plasma energy confinement and instabilities but provides references by which experiments can be compared.

Note that aside from dedicated instruments, there is minimal space around the tokamak vessel to operate new diagnostics and prototype instruments. Competitive formal application and review processes must be followed to obtain time and space to use a tokamak to study fusion plasmas. This dissertation work involves developing a new diagnostic with the goal of becoming a dedicated instrument for STs in the future.
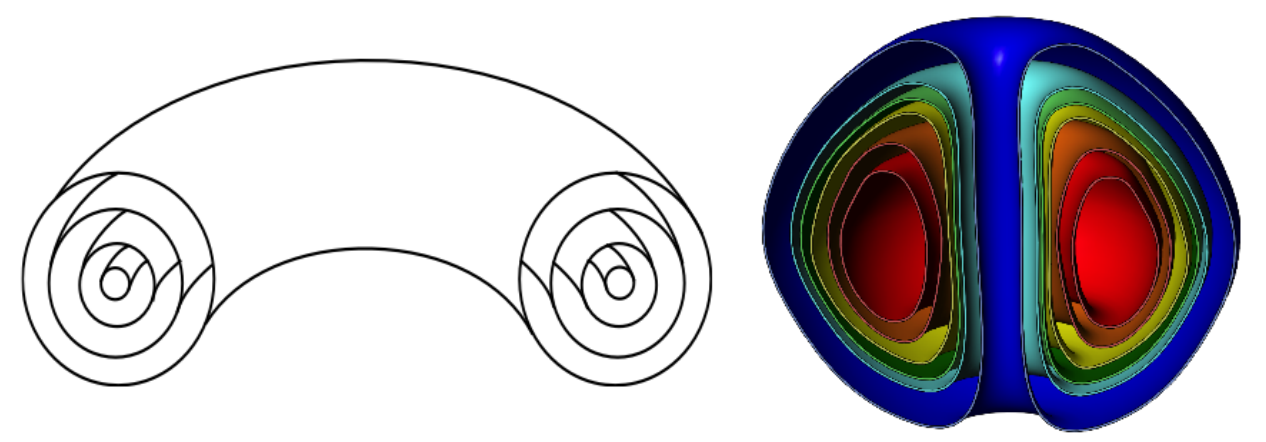

Figure 1.12: The above images are qualitative. The image on the left displays nested flux surfaces for a high aspect ratio plasma, while the image on the right displays nested flux surfaces for a low aspect ratio (such as that created by a spherical tokamak). Magnetic field lines travel along the flux surfaces. The flux surfaces are characterized by constant pressure.

\subsection{Plasma Instabilities}

When a plasma is in equilibrium, the hottest central region of the plasma is where the toroidal current density (which has a radial gradient related to the plasma temperature) has its maximum value. The magnetic field produces nested toroidal surfaces characterized by constant pressure, called flux surfaces (see Figure1.12). The magnetic field lines lie along 
these flux surfaces. Charged particles gyrate along magnetic field lines, they therefore move along these flux surfaces as well. Currents moving in the plasma also move along these flux surfaces.[58]

An outward radial pressure force causes the plasma to expand (because of the kinetic energy of the particles). The outward force is balanced by the inward radial force of the poloidal magnetic field. Therefore for a plasma as a whole to remain in equilibrium, the steady-state solution to the Magnetohydrodynamic (MHD) equations need to satisfy the condition for pressure balance (1.11)

$$
\nabla p=\vec{j} \times \vec{B}
$$

where $\mathrm{p}$ is the plasma pressure and $\vec{j} \times \vec{B}$ is the Lorentz force.[65] Substituting the current density using Ampere's Law (1.12) into the condition for pressure balance yields (1.13).

$$
\begin{gathered}
\vec{j}=\frac{1}{\mu_{0}}(\nabla \times \vec{B}) \\
\nabla p=\frac{1}{\mu_{0}}(\nabla \times \vec{B}) \times \vec{B}
\end{gathered}
$$

Rearranging terms yields the pressure-balance equation ${ }^{1}$

$$
\nabla\left(p+\frac{B^{2}}{2 \mu_{0}}\right)=\frac{1}{\mu_{0}}(\vec{B} \cdot \nabla) \vec{B}
$$

where $B$ is the magnetic field. The ratio of the plasma pressure, or kinetic energy, to the magnetic field energy can be a parameter used to measure the efficiency of using a magnetic field to confine a fusion plasma.[65] The ratio is called $\beta$.

$$
\beta=p\left(\frac{2 \mu_{0}}{B^{2}}\right)
$$

\footnotetext{
${ }^{1}$ The triple cross product identity $A \times B \times C=B \cdot A \cdot C-C \cdot A \cdot B$ is used.
} 
Instabilities within the plasma can perturb or deform the magnetic field such that they can change the balance of forces, $\beta$, within the plasma.[65] Changes in the plasma's current and pressure gradients can destabilize a plasma.[58] Disruptions of flux surfaces can cause changes in the magnetic field configuration thereby causing magnetic fields lines to follow new trajectories because the surface along which they traveled were broken. This in turn can cause changes in ion trajectories which can affect their confinement in the plasma.[58]

Magnetic field oscillations within the plasma can cause instabilities throughout the entire plasma. These oscillations, also called Mirnov oscillations, can be measured and observed through coils (small magnetic pickup loops) placed outside of the plasma.[58] Additionally, soft x-ray emissions can leave the plasma such that oscillations which are sawtooth in shape can be observed.[58] When sawtooth oscillations, also called relaxation oscillations, occur in the central region of the plasma, thermal energy is transported to the outer edge (colder) region of the plasma. These oscillations can also be observed in the plasma temperature and density. Furthermore, fishbone (FB) bursts, or events, can arise during the NBI as they are related to fast ions created during this heating phase which can perturb the magnetic field.[58] FBs can be observed through Mirnov coils. These instabilities can lead to degradation of the current drive and loss of ion confinement. Examples of sawtooth oscillations and FBs during a plasma will be further discussed in Chapter 6.

Oscillations within a plasma that can lead to instabilities which disrupt a plasma's equilibrium, trigger other instabilities, disrupt the toroidal plasma current, and affect plasma temperature are an important focus within fusion research. The relationships between instabilities to each other, plasma particles, and plasma parameters are complex and not completely understood in the plasma physics and fusion research communities. This highlights the importance of empirical methods to observe the effects of oscillations and instabilities. 


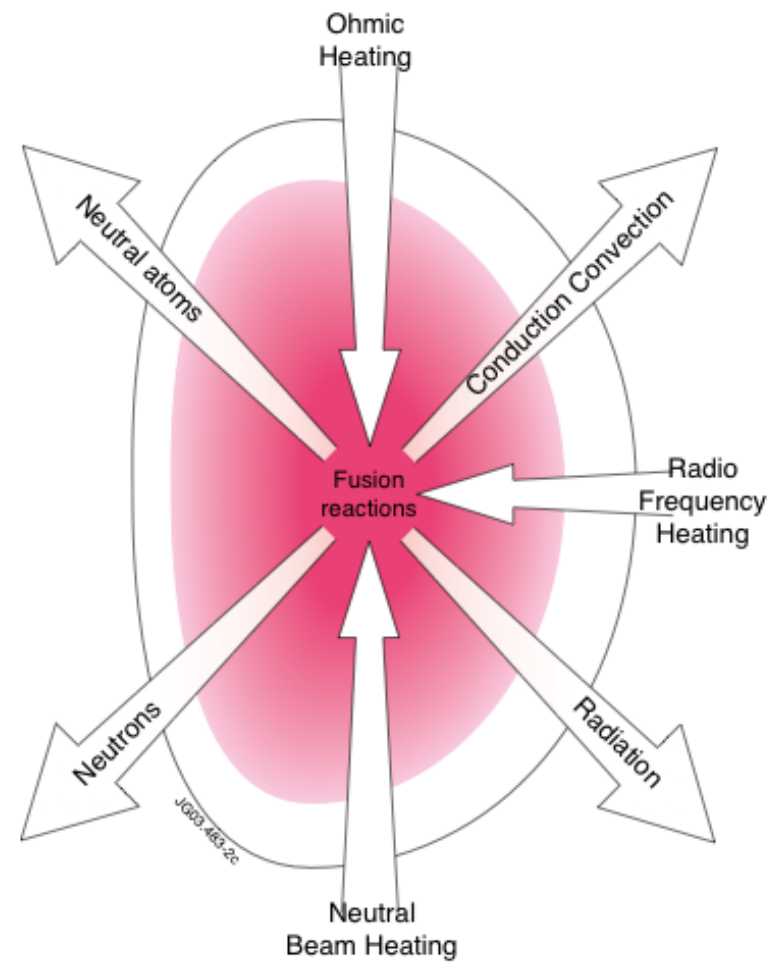

Figure 1.13: The energy confinement time is related to balancing the energy lost from the plasma and the external heating power supplied to the plasma.[41]

Motivation for Studying Plasma Instabilities Designs for future nuclear fusion power reactors rely on the ability to create a steady-state stable plasma as a medium with which to sustain nuclear fusion reactions. A plasma, however, continuously loses power (1.16). Energy leaves the plasma through radiation, thermal conduction and convention, and particle loss (see Figure 1.13).[36] Over time this power loss is replenished through external heating (inductive and non-inductive) methods previously discussed. The balancing of lost power, $P_{L}$, and externally supplied power, $P_{S}$, yields the energy confinement time which is referred to as $\tau_{E}$.

$$
\begin{gathered}
P_{L}=\frac{E_{\text {Plasma }}}{\tau_{E}} \rightarrow \tau_{E}=\frac{E_{\text {plasma }}}{P_{L}} \\
P_{S}=P_{L} \rightarrow \tau_{E}=\frac{E_{\text {Plasma }}}{P_{S}}
\end{gathered}
$$


The plasma's energy confinement time (1.17) describes the minimum amount of time plasma ions need to retain their energy and remain within the plasma to undergo a fusion reaction.[58] It is a ratio between the plasma energy (units of Joules) and the amount of heating power (units of W or Joules per second) necessary to sustain the plasma's energy. Plasma ions, especially beam ions, must remain in the plasma at least this long in order to have the plasma stored energy remain steady or increase.

Ignition is the point when a plasma no longer requires external power input (through heating) to balance its loss of power because the plasma has sufficient internal heating provided from ion collisions. A criterion, known as the Lawson Criterion, for a tokamak to reach ignition is related to the energy confinement time and can be described by the relationship in (1.18). It is related to the plasma density, core temperature, and $\tau_{E}$.[58][36]

$$
n T \tau_{E}>5 \times 10^{21} \mathrm{keVsm}^{-3}
$$

In order for a fusion reactor to generate power, this relationship needs to be satisfied.[36] The second main criterion used to discuss fusion reactor design is $\mathrm{Q}$, a ratio of fusion reactor power output to the external power input.

Efforts of mitigating and controlling plasma instabilities contribute towards enabling tokamaks to reach ignition. Learning how instabilities affect the distribution and loss of plasma particles can help develop techniques to mitigate instabilities, which can improve non-inductive methods of current drive and the plasma energy confinement time.

\subsection{Methods to Study Plasma Instabilities}

High temperatures within the plasma preclude inserting instruments, or diagnostics, directly into the plasma to study its properties. Aside from damaging the instruments, inserting them into the plasma could add impurities thereby negatively affecting a plasma's 


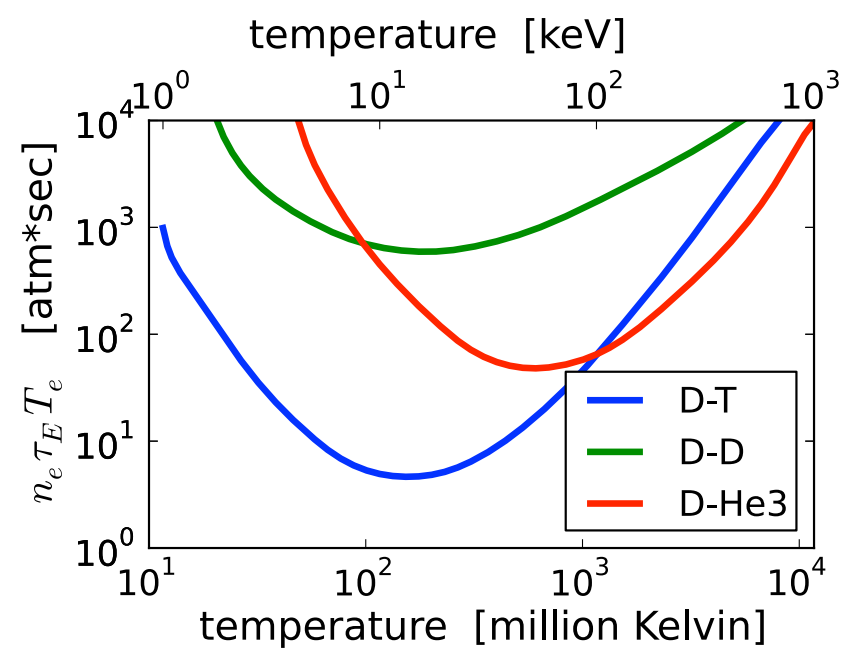

Figure 1.14: This graph displays the Lawson Criterion for nuclear fusion reactions between hydrogen isotopes and helium isotopes. A tokamak that is able to satisfy conditions above the plotted curves can reach ignition.[34]

energy confinement. Therefore, the best way to study plasma parameters is to detect particles and radiation leaving the plasma. The technique of detecting charged particles will be the focus of this dissertation work, see Figure 1.15 and Figure 1.16.

Charged Particle Detection Earlier experiments explored various detection techniques to measure ion energy spectra, rates at which they were emitted from the plasma, and from where in the plasma they were emitted. Finding a balance between energy, spatial, and timing resolution enabled the discussion of ion emission profiles. To carry out these investigations, detection experiments took advantage of charged particle motion in the plasma's magnetic field. As ions gyrate along the magnetic field lines, a vertical poloidal drift can cause them to leave the plasma and travel towards the vessel wall, thereby depositing their energy into a detector placed within the vessel walls.

Chrien, Kaita, and Strachan (1983) measured energy spectra for $3 \mathrm{MeV}$ DD fusion protons (250 keV resolution) with silicon surface barrier detectors in the Princeton Large Torus (PLT). Relations between changes in the proton energy spectrum and the plasma ion 


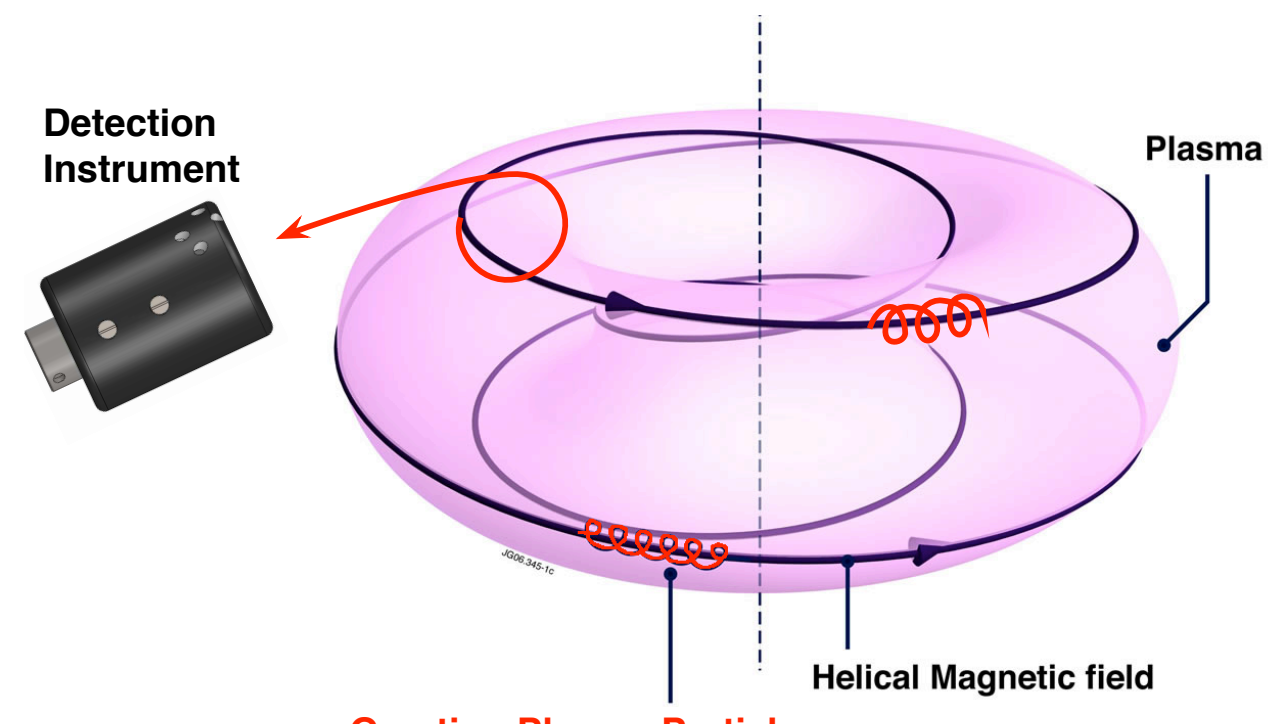

Gyrating Plasma Particle

Figure 1.15: The above qualitative image represents a plasma created by a conventional tokamak. Charged particles gyrating along helical magnetic field lines in a toroidal plasma can leave the plasma and hit a detector if the gyroradii of their trajectories are large enough.[37]

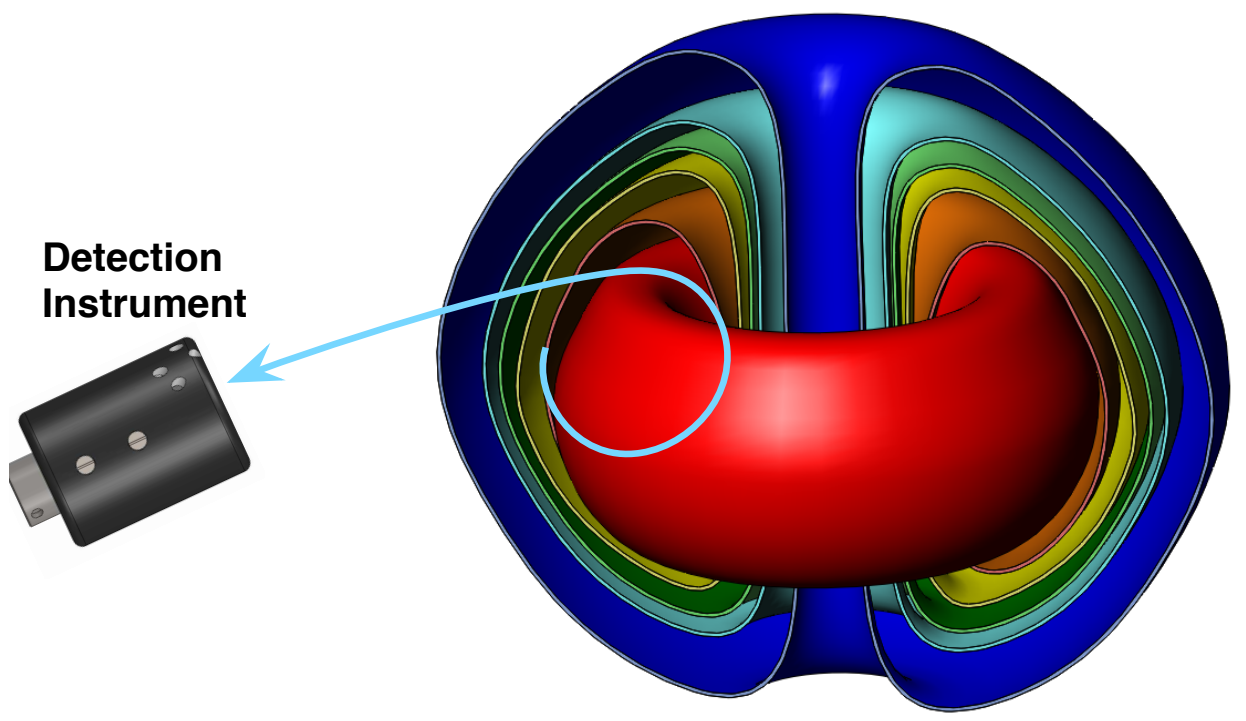

Figure 1.16: The above qualitative image is of nested constant flux surfaces within a plasma created by a spherical tokamak. Charged particles gyrating along helical magnetic field lines in a spherically-shaped toroidal plasma can leave the plasma and hit a detector if the gyro-radii of their trajectories are large enough. The red inner surface represents the hot central region of the plasma. 
temperature were studied during various plasma heating scenarios. Lower resolution (2 $\mathrm{MeV}$ ) energy measurements were also obtained for the $14.7 \mathrm{MeV}$ proton.[17] Heidbrink and Strachan (1985) subsequently used collimated silicon surface barrier detectors to measure both energy spectra and emission rates for $3 \mathrm{MeV}$ DD fusion protons in the PLT. Integrating energy spectra in $10 \mathrm{~ms}$ intervals and graphing values as a function of changing toroidal plasma current showed promise for this type of system to study changes in plasma ion temperature.[47] During experiments at the Tokamak Fusion Test Reactor (TFTR), Strachan (1986) used an array of silicon surface barrier detectors to measure the $14.7 \mathrm{MeV}$ $\mathrm{DHe}^{3}$ fusion proton (1.19) pulse-height spectra. Though because of installation constraints (wide collimation aperture) for the experiment, the low spatial resolution for the energy spectra prevented a discussion of a proton emission profile.[49]

$$
D+H e^{3}=P(14.7 M e V)+H e^{4}(3.7 M e V)
$$

Additionally at the TFTR, Zweben (1986) made use of scintillator detectors to create energy spectra (from light emission) from 3.5 MeV DT fusion $\mathrm{He}^{4}$ ions (1.8) and $1 \mathrm{MeV} \mathrm{DD}$ fusion tritons; despite broad pulse widths (low energy resolution) there were high signal to noise ratios.[25] Furthermore, Martin et al. (1987) studied the $14.7 \mathrm{MeV} \mathrm{DHe}^{3}$ fusion proton energy spectra and its effects on fast ion confinement at the Joint European Torus (JET) during sawtooth instabilities.[14]

At the Axially Symmetric Divertor Experiment (ASDEX), Bosch (1990) used an array of silicon barrier detectors, attached to the end of a mechanical arm, to measure peak width broadening on the energy spectra of $1 \mathrm{MeV}$ DD fusion tritons and $3 \mathrm{MeV}$ fusion protons during different plasma heating scenarios (both inductive and non-inductive heating methods). The geometry of the tokamak and size of the gyro-radii are such that the protons and tritons escaped the plasma through a poloidal vertical drift. The experimental design, 
however, prevented an analysis of the spatial resolution of where the protons and tritons originated in the plasma.[45] Features of $1 \mathrm{MeV}$ tritons and $3 \mathrm{MeV}$ protons were also identified by Darrow, Boivin, and Zweben (1992) in scintillation detector measurements taken with a Fast Ion Probe in the TFTR.[27] Further expanding on scintillation-based detection techniques, Darrow, et. al (1995) detected 3.5 MeV DT fusion $\mathrm{He}^{4}$ ions in the TFTR. Ions would hit a collimated scintillator which then emitted light. The intensity of light and its spatial distribution were recorded with photomultiplier tubes and sensitive videocameras. Higher rates of incident particles hitting the scintillator resulted in greater light intensity. Helium-4 ( $\left.\mathrm{He}^{4}\right)$ signals produced by the scintillation detectors, however, could be as low as $15 \%$ to $25 \%$ of the total signal (as neutrons are detected in the system well).[26] Boivin and Petrasso's (1995) charged fusion product spectrometer (at the Alcator CMOD) used bakeable silicon detectors to study local regions of the plasma ion temperature through energy spectra of $14.7 \mathrm{MeV} \mathrm{DHe}^{3}$ fusion protons. The design included the possibility of detecting $3 \mathrm{MeV}$ DD fusion protons and $1 \mathrm{MeV}$ DD fusion tritons as well. The experiment mentioned the future use of EFIT equilibrium codes for improved calculations of particle trajectories from where they originate in the plasma to their incidence on the detectors; this will be further discussed in Chapter 2.[46]

Fast Ion Diagnostics The advantage of the ST for detecting charged fusion products is considerably smaller toroidal magnetic fields leading to gyro-orbits for the fusion products of the order of the plasma size.

Owen et al.'s (2013) work with the Fast Ion D-Alpha Spectrometer (FIDA) installed at MAST detects light emitted from charge exchange interactions between fast ions in the plasma and re-neutralized beam ions. The density (in local regions of the plasma) of 
passing and fast ions trapped in the plasma can be extracted from these measurements.[23] Similar to the MAST spectrometer diagnostic, the NSTX has a Fast-Ion D-Alpha Diagnostic in which Podesta et al. (2008) has studied fast ion confinement as well.[22]

The Solid State Neutral Particle Analyzer (SSNPA) installed at NSTX makes use of silicon photodiode detectors (as opposed to scintillation detectors) to measure the energy spectra (range from $30 \mathrm{keV}$ to $100 \mathrm{keV}$ ) and rate of neutral particles, that were neutralized and emitted light during charge exchange interactions, escaping from local regions of the plasma. Liu et al.'s (2006) study discussed improvements in mitigating noise caused by electromagnetic interference which resulted in improved energy (10 keV) and timing resolution ( $2 \mathrm{~ms})$. The data acquisition system included charge-sensitive preamplifiers and high-speed digitizers, after which pulse-height analysis was applied to recorded detector signals.[24] The work of detecting charge exchange light emission to study fast ion loss has been continued with new improvements to neutral particle analyzer diagnostics in both the DIIID tokamak and NSTX.[28]

Neutron Detection in Spherical Tokamaks Instead of directly detecting charged fusion products or light emitted from charge exchange interactions between beam and plasma ions, another technique is to detect neutrons such as $2.45 \mathrm{MeV}$ DD fusion neutrons and 14.1 MeV DT fusion neutrons.

Barnes and Strachan's (1990) work with the neutron spectrometer, installed at the TFTR, describes two components of the diagnostic. Count rates of 2.45 MeV DD fusion neutrons were indirectly measured with an $\mathrm{He}^{3}$ ionization chamber (1.20).

$$
H e^{3}+N=T+P
$$

Rates for the 14.1 MeV DT fusion neutrons are indirectly measured with a NE213 proton recoil spectrometer, in which signals for recoiled protons (from elastic scattering with 


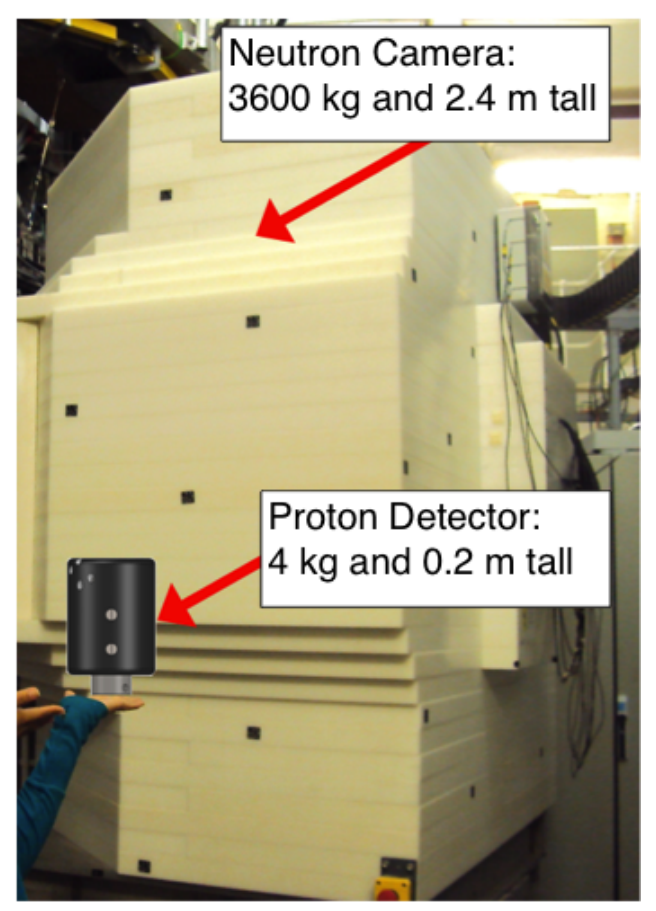

Figure 1.17: Proton detection techniques allow for a relatively smaller and lightweight mechanical housing design when compared with neutron detection techniques. Note that a scaled CAD drawing of the PD diagnostic was Photoshopped into the above image and used with approximations for the weight and dimensions of the instruments to provide a qualitative reference.

neutrons) were detected.[29] Stammers and Loughlin (2006) describe the primary neutron diagnostic at MAST, which is a fission chamber based on the $U^{235}$ fission reaction (1.7). These neutron rates provide information about the total, or global, rate of neutrons emitted from the plasma with a resolution of $10 \mu \mathrm{s}$; it does not provide information about local regions of the plasma. [30]

Collimated neutron detection (CND) diagnostics use liquid scintillation detectors to determine neutron emission rates in small, local, regions of the plasma. Cecconello et al.'s (2014) research involved creating neutron emission profiles from a four-channel $2.45 \mathrm{MeV}$ DD fusion neutron detection instrument installed at MAST. [20] Klimek et. al (2014) compared local neutron emission to global neutron emission rates as well as simulations to study various MHD instabilities.[13] 


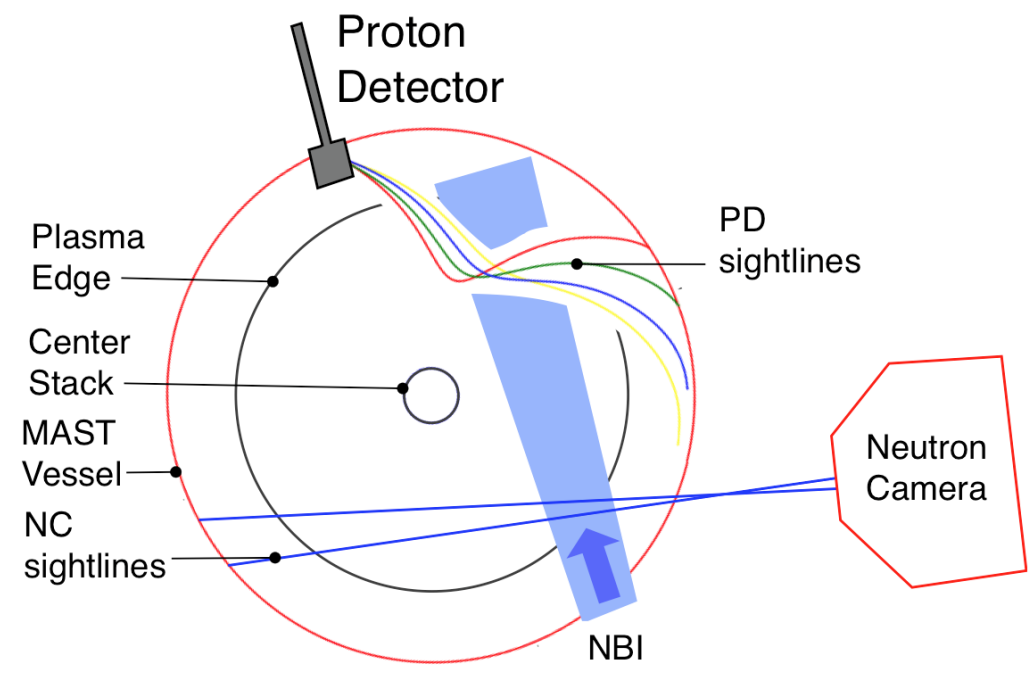

Figure 1.18: Above is a qualitative image with a projection of Proton Detector and Neutron Camera toroidal sightlines at the Mega Amp Spherical Tokamak (MAST). The original image is from Cecconello et al.'s work (2012).[19]

Motivation for New Spherical Tokamak Diagnostics As previously mentioned, the highest yield of DD fusion reaction products result from reactions of beam ions with thermal plasma ions (BT) during the NBI heating phase of the plasma. Therefore, studying DD fusion products during this phase is a useful tool to measure the fast ion distribution throughout the plasma. Collimated neutron detection (CND) systems can be designed to detect the $2.5 \mathrm{MeV}$ neutron emitted from these DD fusion reactions. Recalling the probability of both branches of the DD fusion reaction infers that measuring the number of 2.5 $\mathrm{MeV}$ neutrons provides similar information as measuring the $3 \mathrm{MeV}$ proton emission rate. My dissertation research discusses a diagnostic developed for MAST to directly measure rate and energy information from emitted DD charged fusion products. Though the diagnostic detects both protons and tritons (1.9), the data analysis and discussion will focus on protons; this will be further discussed in Chapter 5.

Though CND instruments have been successfully used in STs, not every tokamak has the physical space required (around the vessel) to install such an instrument. Limited space 
for instruments affects STs worldwide, such as the MAST-Upgrade (MAST-U) and NSTXUpgrade (NSTX-U), which are installing a second neutral beam injection system to increase the density and energy of their plasmas. For example, the NSTX-U at the PPPL will not have space to install a CND system and is therefore interested in the instrument described in this dissertation. The reduced size of silicon surface barrier detectors used for charged particle detection (as opposed to neutrons) makes the design of a relatively compact instrument possible, see Figure 1.17.

Aside from physical dimensions of the instrument, the design for a four-channel charged fusion product detection system costs around $\$ 50,000$. The mounting of a charged fusion product instrument on a mechanical arm (which then inserts the instrument into the tokamak vessel towards the plasma) allows the instrument to sample different regions of the plasma by remotely moving the position of the mechanical arm along or about its axis. Because of its weight and size, to sample different regions of a plasma with a CND, the entire instrument needs to be mounted on a custom railing system; this would require more space than the NSTX-U can provide. Additionally, blank vacuum flanges between the plasma and diagnostic may be required for each CND sightline, which excludes those ports from being used for other diagnostics.

The installation of a charged fusion product instrument on a mechanical arm, however, necessitates an installation of the instrument into the ultra high vacuum (UHV) environment of the tokamak vessel. Designing an instrument for operation in a UHV environment, and to withstand temperatures close to the plasma as well as baking temperatures, can involve more design constraints than operating outside of the vessel. A charged particle instrument needs to be inside of the vessel because protons and tritons cannot travel through the vessel wall to outside of the tokamak; they would deposit all of their energy on the 
inside wall of the vessel. Owing to the curved trajectories of these charged fusion products, the simulations for spatial locations from where they originate in the plasma are highly dependent upon an accurate reconstruction of the plasma's magnetic field.

The diagnostic designed for this dissertation work was developed initially for NSTX but was later installed at MAST at the Culham Center for Fusion Energy (CCFE) in the United Kingdom. ${ }^{1}$ Because MAST already had a CND system installed, adding another instrument to detect different products from DD fusion reactions was an opportunity to compare data taken from complementary instruments (see Figure 1.18). The presence of a fission chamber (to infer total neutron rates produced) and CND (sampling local regions of the plasma) was an invaluable benefit in validating this charged fusion product diagnostic. Cecconello et al.'s (2015) recent work combines data taken with the FIDA, fission chamber, $\mathrm{CND}$, and PD diagnostics to demonstrate the benefit of using multiple complementary instruments to study plasma oscillation and instability effects on the fast ion distribution in the plasma.[12]

\subsection{Dissertation Research Overview}

The goal of my dissertation research is to design, test, and validate an instrument to study plasma oscillations (which can lead to instabilities) through charged particles emitted from the plasma during NBI heating. For short, this charged fusion products diagnostic is called the Proton Detector. The Proton Detector (PD) was the first diagnostic to detect $3 \mathrm{MeV}$ protons and $1 \mathrm{MeV}$ tritons emitted from DD fusion reactions (1.9) inside of an ST plasma. Unlike experiments conducted in conventional tokamaks, which took advantage of poloidal

\footnotetext{
${ }^{1}$ Because of an unplanned early shutdown of NSTX to begin upgrades, the instrument was not installed at the NSTX. A new instrument, the PD, was designed for MAST. Subsequently, after the success of the PD installed at MAST a new version of the instrument is being designed for the NSTX-U at the PPPL. The MAST diagnostic will be the focus of this dissertation.
} 
drifts, the protons and tritons reach the PD's detector before completing one full gyroorbit. The large gyro-radii of these particles, compared with the geometry of the spherical tokamak, allow DD fusion protons and tritons to leave the plasma relatively unconfined. ${ }^{1}$

While other diagnostics will indirectly study these charged particles through: Coulomb interactions, detecting other charged particles, or detecting neutral particles, the PD directly detects these protons and tritons. The particles' curved trajectories, however, complicate the calculation of their positions within the plasma because of a heavy dependence on the accuracy of the plasma's constructed magnetic field configuration. The benefit of further developing the PD lies in its relatively compact size and low cost compared to CND systems. As STs continue to increase their plasma density, hence needing to add a secondary neutral beam injection system, some facilities that do not have the space for CND systems will need another method to measure DD charged fusion product loss from the plasma.

Charged particle energy spectra and rate measurements can be a useful method to observe oscillations and instabilities within a plasma. Studying their effects on a plasma's fast ion distribution (spatially and temporally) in STs contribute to efforts in creating future nuclear fusion power reactors.

\footnotetext{
${ }^{1}$ For example, the gyro-radius for a $3 \mathrm{MeV}$ proton in a $0.5 \mathrm{~T}$ toroidal magnetic field is $0.51 \mathrm{~m}$. This value is comparable to a plasma minor radius of $0.65 \mathrm{~m}$ at MAST. If a proton had an energy of $60 \mathrm{keV}$, however, its gyro-radius would only be $0.07 \mathrm{~m}$. More gyro-radii values are listed in Chapter 2.
} 


\section{CHAPTER 2}

\section{DIAGNOSTIC MECHANICAL DESIGN}

This chapter concerns the planning and design of the mechanical components of the instrument, which are informed by a simulation taking into account the magnetic field configuration of the plasma.

\subsection{Orbit Code}

The PD diagnostic's technique of detecting charged particles to study plasma oscillations and instabilities needs to take into account where plasma particles are emitted after being produced by DD fusion reactions. Each individual channel in the PD diagnostic views a different curved sightline in the plasma. These sightlines are also called curved particle trajectories, or orbits. A modified orbit code (based on a version of the Lorentz ORBIT Code ${ }^{1}$ which was provided by PPPL collaborators) was used to calculate the time-reversed proton and triton orbits reaching each detector. Orbit codes calculating time-reversed particle orbits, through numerically integrating the Lorentz force acting on particles, are commonly used in experimental fusion research. General orbit code inputs include particle properties such as charge and energy, and detector orientations with respect the center of the plasma.

Proton and triton, or ion, orbits depend on the plasma's internal and external magnetic field configuration, and are highly dependent on the plasma's toroidal magnetic field.[49] Assumptions such as a constant toroidal magnetic field in time and a constant plasma current in time are used to calculate particle orbits. The orbit code also assumes that: the initial particle energy (and therefore absolute value of the velocity) is fixed, there is toroidal symmetry throughout the plasma, and the DD fusion reactions are isotropic.[45]

\footnotetext{
${ }^{1}$ The original PPPL Lorentz ORBIT code is described in Felt et al.'s work (1990).[42]
} 
Each point along a particle's orbit corresponds to a point in the particle's phase space in the plasma.[45] Particle motion which is described by Hamiltonian equations of motion preserves the conservation of phase space volume for a system in time.[59][45] Consequently, the orbit code numerically calculates the ion trajectories assuming that the phase space distribution function from the ion birth in the plasma volume to the ion incidence in the detector volume remains constant. The six-dimensional phase space is characterized by space and momenta coordinates $\vec{r}$ and $\vec{v}$. The conservation of phase space also signifies that the particle density in phase space remains the same. A constant particle density indicates that no particles are lost through traveling from the plasma volume to the detector volume, meaning the particles do not undergo collisions. The assumption of all particles reaching the detector originating from the plasma volume without undergoing collisions is a consequence of these Hamiltonian equations of motion in the code.

Equilibrium Fitting Results from the Equilibrium Fitting (EFIT) code are used by the orbit code simulation. The orbit codes uses EFIT's reconstruction of the equilibrium magnetic field configuration to calculate particle trajectories.[56] The EFIT code iteratively fits solutions to the plasma's equilibrium equation. A condition for equilibrium (2.1), discussed in Chapter 1 , requires a balance between the magnetic force, $\vec{j} \times \vec{B}$, and the plasma pressure, $p$.

$$
\nabla p=\vec{j} \times \vec{B}
$$

Through expanding the current density, $\vec{j}$, and magnetic field, $\vec{B}$, in terms of their poloidal and toroidal components, (2.1) is expressed in a form called the Grad-Shafranov equation,

$$
R \frac{\partial}{\partial R} \frac{1}{R} \frac{\partial \Psi}{\partial R}+\frac{\partial^{2} \Psi}{\partial z^{2}}=-\mu_{0} R^{2} p^{\prime}(\Psi)-\mu_{0}^{2} f(\Psi) f^{\prime}(\Psi)
$$


where $p^{\prime}$ and $f f^{\prime}$ are arbitrary functions of the poloidal flux, and $\mathrm{R}$ and $\mathrm{z}$ are the poloidal coordinates.[58] The EFIT reconstruction code uses experimental data ${ }^{1}$ as constraints to adjust $p^{\prime}$ and $f f^{\prime}$ in order to numerically solve (2.2) for a single time slice.[57] Simulation codes using EFIT results to calculate time-reversed particle orbits are commonly used in experimental fusion research.[44][45][46][47] See Figure 2.1 for a graphical application of using EFIT results.

1.

${ }^{1}$ Data, such as pitch angles indicating the direction of the magnetic field, are provided by dedicated diagnostics mentioned in Chapter 


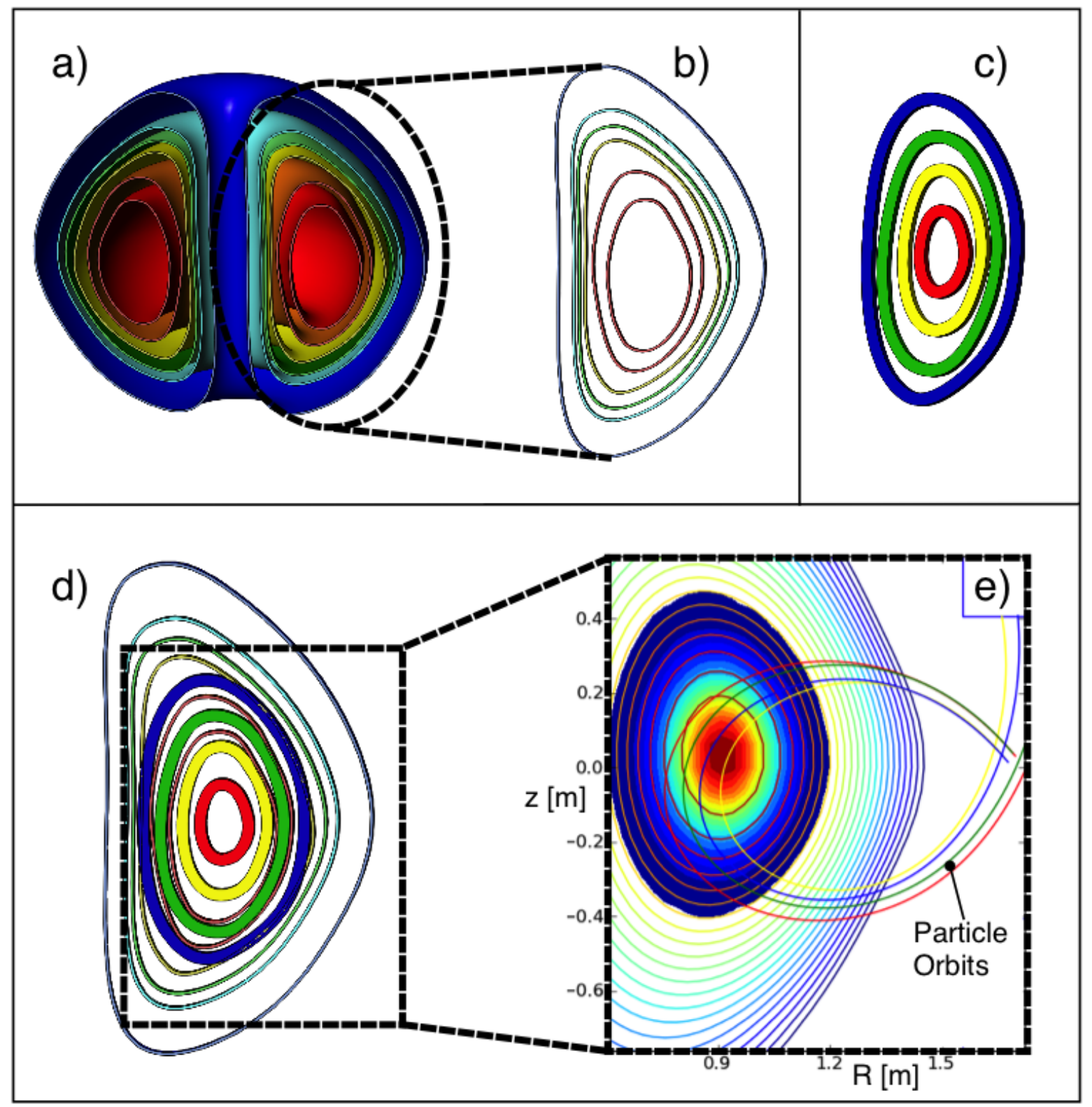

Figure 2.1: Figures a) through d) in the images above are qualitative. Figure a) is a cross-sectional three-dimensional view of nested constant flux surfaces within the plasma. Figure b) is a two-dimensional vertical, or poloidal, slice of the flux surfaces. Results from the EFIT code provide information about this poloidal flux. Figure $\mathrm{c}$ ) is the simulated two-dimensional poloidal particle emissivity, $\mathrm{S}$, for regions in the plasma. This example $\mathrm{S}$ has an elliptical shape because it is based on a gaussian function, where the color red represents the highest emission and blue represents the lowest emission. Figure d) combines the poloidal flux in b) and the particle emissivity in c). Figure e) is an example of the orbit's code graphical output in which poloidal particle orbits are plotted over d). The poloidal coordinates of the graphical output are R ( $\mathrm{m}$ from the center of the tokamak) and $\mathrm{z}$ ( $\mathrm{m}$ above and below the plasma mid-plane). 


\begin{tabular}{|c|c|c|c|c|c|}
\hline Particle & $\begin{array}{c}\text { Energy } \\
{[\mathrm{MeV}]}\end{array}$ & $\begin{array}{c}\text { Nucleon } \\
\text { Number }\end{array}$ & $\begin{array}{c}\text { Velocity } \\
{\left[\mathrm{ms}^{-1}\right]}\end{array}$ & $\begin{array}{c}\text { Momentum } \\
{\left[\mathrm{kgm} s^{-1}\right]}\end{array}$ & $\begin{array}{c}\text { Momentum } \\
{\left[\mathrm{MeV} c^{-1}\right]}\end{array}$ \\
\hline Proton & 3.02 & 1 & $2.41 \times 10^{7}$ & $4 \times 10^{-20}$ & $\sim 75$ \\
\hline Triton & 1.01 & 3 & $8.05 \times 10^{6}$ & $4 \times 10^{-20}$ & $\sim 75$ \\
\hline Helium-3 & 0.82 & 3 & $7.26 \times 10^{6}$ & $3.6 \times 10^{-20}$ & $\sim 67$ \\
\hline Neutron & 2.45 & 1 & $2.17 \times 10^{7}$ & $3.6 \times 10^{-20}$ & $\sim 67$ \\
\hline
\end{tabular}

Table 2.1: This table contains calculated characteristic values for products from DD reactions.[58]

Characteristic Ion Values The $3 \mathrm{MeV}$ proton and $1 \mathrm{MeV}$ triton have nearly the same momentum thereby following nearly the same orbits while leaving the plasma. Figure 2.2 displays characteristic proton and triton orbits projected onto the poloidal plane (coordinates $\mathrm{R}$ and $\mathrm{z}$ ) and toroidal plane (coordinates $\mathrm{X}$ and $\mathrm{Y}$ ). As these ions gyrate along magnetic field lines in the plasma, their gyro-frequency as introduced in Chapter 1 can be expressed as

$$
\omega_{g}=\frac{Z e B_{T}}{m}\left[s^{-1}\right] \quad \text { or } \quad f_{g}=\frac{\omega_{g}}{2 \pi}[H z]
$$

where $\mathrm{Z}$ is the number of protons in the ion, e is the electronic charge, $B_{T}$ is the toroidal magnetic field, and $\mathrm{m}$ is the ion mass. Table 2.1 and Table 2.2 list calculated characteristic properties for these ions. Velocities from Table 2.1 and (2.3) are used to calculate the gyro-radius, or Larmor radius, of an ion's orbit for a given toroidal magnetic field value,

$$
R_{g}=\frac{v}{\omega_{g}}
$$

where the velocity perpendicular to the magnetic field contributes to the gyro-motion, $v=$ $v_{\perp}$, and a constant toroidal magnetic field is assumed. The gyro-radii for protons and tritons in the MAST spherical tokamak (ST) are typically on the order of $1 \mathrm{~m}$ which allows them to leave the plasma before completing one full gyro-orbit. 


\begin{tabular}{|c|c|c|c|c|}
\hline Particle & $B_{T}[$ Tesla] & $\omega_{g}\left[s^{-1}\right]$ & $f_{g}[\mathrm{MHz}]$ & $R_{g}[\mathrm{~m}]$ \\
\hline \multirow{3}{*}{ Proton } & 0.5 & $47.75 \times 10^{6}$ & 7.6 & 0.51 \\
\cline { 2 - 5 } & 0.6 & $57.3 \times 10^{6}$ & 9.12 & 0.42 \\
\hline \multirow{3}{*}{ Triton } & 0.5 & $15.92 \times 10^{6}$ & 2.53 & 0.51 \\
\cline { 2 - 5 } & 0.6 & $19.1 \times 10^{6}$ & 3.04 & 0.42 \\
\hline Helium-3 & 0.5 & $31.8310^{6}$ & 5.07 & 0.23 \\
\cline { 2 - 5 } & 0.6 & $38.2 \times 10^{6}$ & 6.08 & 0.19 \\
\hline
\end{tabular}

Table 2.2: This table contains values for the gyro-radii of protons, tritons, and helium-3 ions.[58]

Particle Emissivity The particle emission from the plasma, or emissivity, can be represented as a function of several parameters such as the plasma's poloidal magnetic flux and the poloidal position within the plasma. An example function used for the particle emissivity, $\mathrm{S}$, is a power law (2.5)

$$
S=\psi_{\text {Relative }}^{\lambda}
$$

where $\lambda$ is a fitting parameter and $\psi_{\text {Relative }}$ is the magnetic flux (poloidal projection) relative to the magnetic axis of the plasma. The relative poloidal magnetic flux is defined such that $\psi_{\text {Relative }}=1$ at the magnetic axis and $\psi_{\text {Relative }}=0$ at the plasma edge.

When the orbit code calculates the simulated particle emission, it will use an input function such as this S. ${ }^{1}$ Different functions can be used by the orbit code to calculate $S$ along individual detector channel orbits, which will be further discussed in Chapter 6 .

Detector Collimation The number of orbits intersecting the active area of the detector is influenced by the effective opening of the collimator in front of the detector.[49] A bundle of orbits, or orbit bundle, has a distribution of sightline directions that are incident with the detector active area.[45] Figure 2.2 displays the four PD channel orbit trajectories. Note that though the poloidal trajectories graphed from the orbit code are central trajectories,

\footnotetext{
${ }^{1}$ Note that the use of $\mathrm{S}$ as a function of $\psi$ implies a constant particle emission along the nested surfaces of constant flux in the plasma.[54]
} 

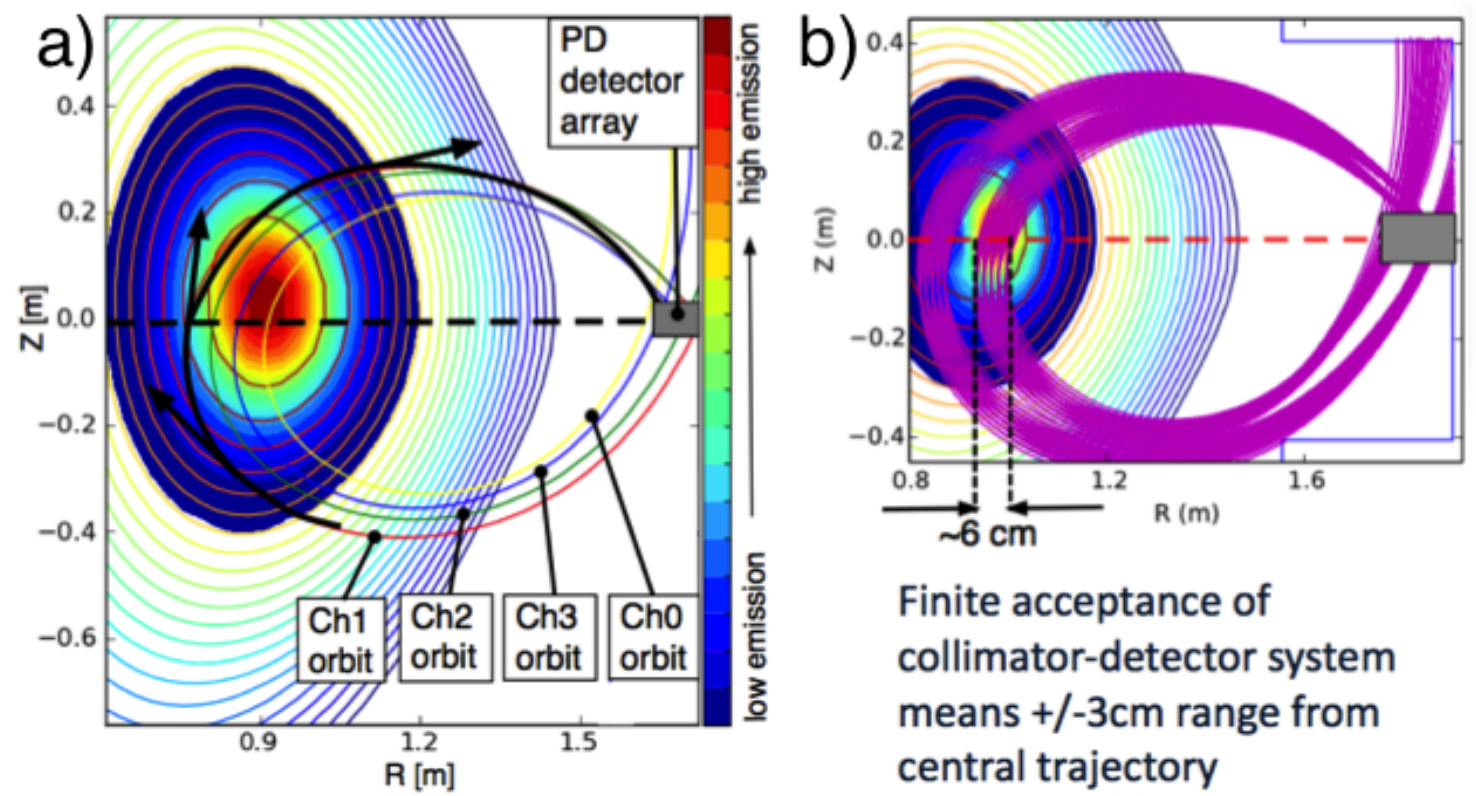

\section{Finite acceptance of collimator-detector system means $+/-3 \mathrm{~cm}$ range from central trajectory}
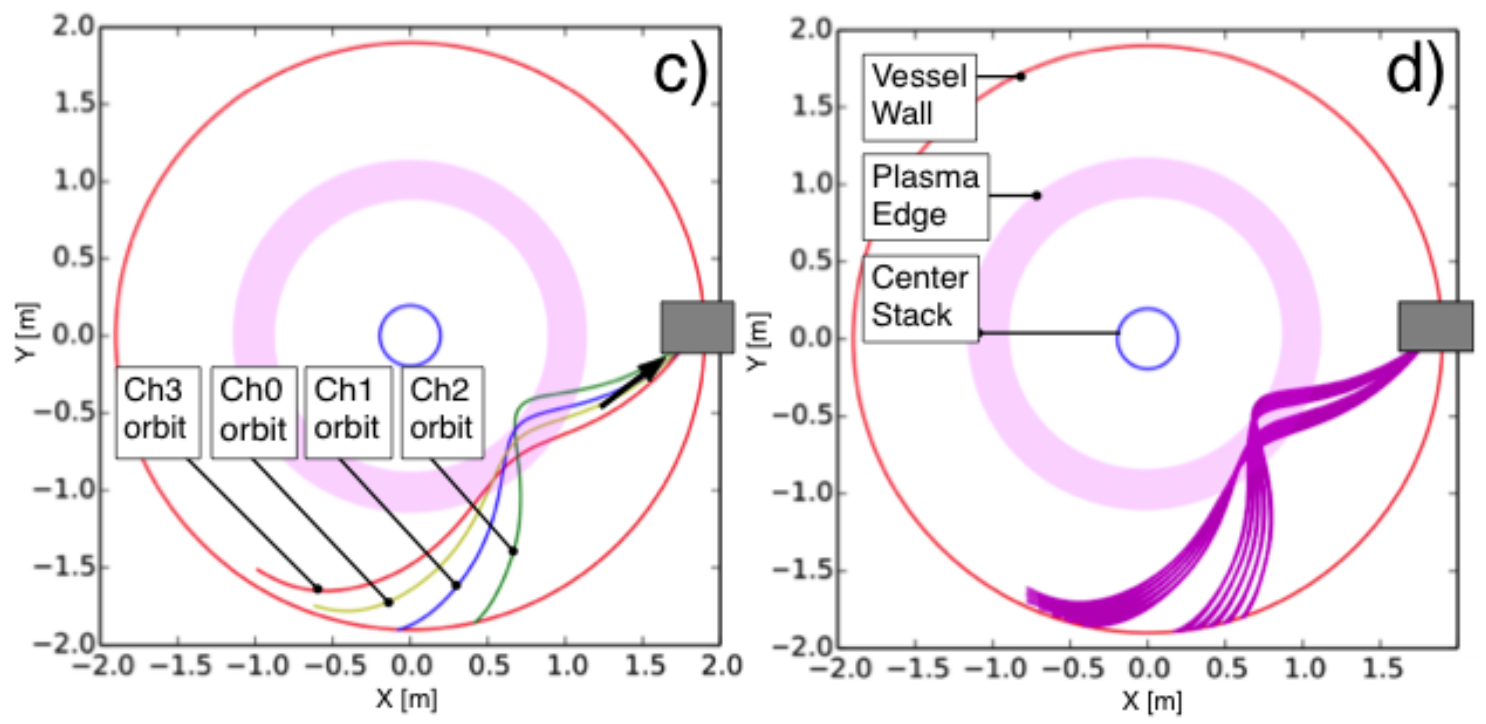

Figure 2.2: The above images are graphical outputs of the orbit code which display particle orbits. Figure a), from Perez et al., Rev. Sci. Instrum. 85, 11D701 (2014), displays the poloidal projection of the central orbits plotted over the poloidal flux and emission regions (coordinates of $\mathrm{R}$ and z).[55] Figure b) shows the corresponding thickness of the orbit bundle incident on the detector channels. Figure c) displays the toroidal projection of the central orbits (coordinates of $\mathrm{X}$ and $\mathrm{Y}$ ). Figure d) shows the corresponding thickness of the orbit bundle incident on the detector channels. The thickness of the orbit bundle is directly associated to the collimator opening in front of the detector. 
there is a thickness (for a range of radial positions) of particle orbits that can enter the detector. The circular $3.8 \mathrm{~mm}$ collimator diameter opening with a length of $38 \mathrm{~mm}$, influences the $+/-3 \mathrm{~cm}$ (from the central trajectory) spatial resolution for a radial value along the $\mathrm{z}=0$ plane, or mid-plane, of the plasma.[55] The average angular acceptance for the detector collimator geometry is $+/-2.86^{\circ}$, see Figure 2.3 .
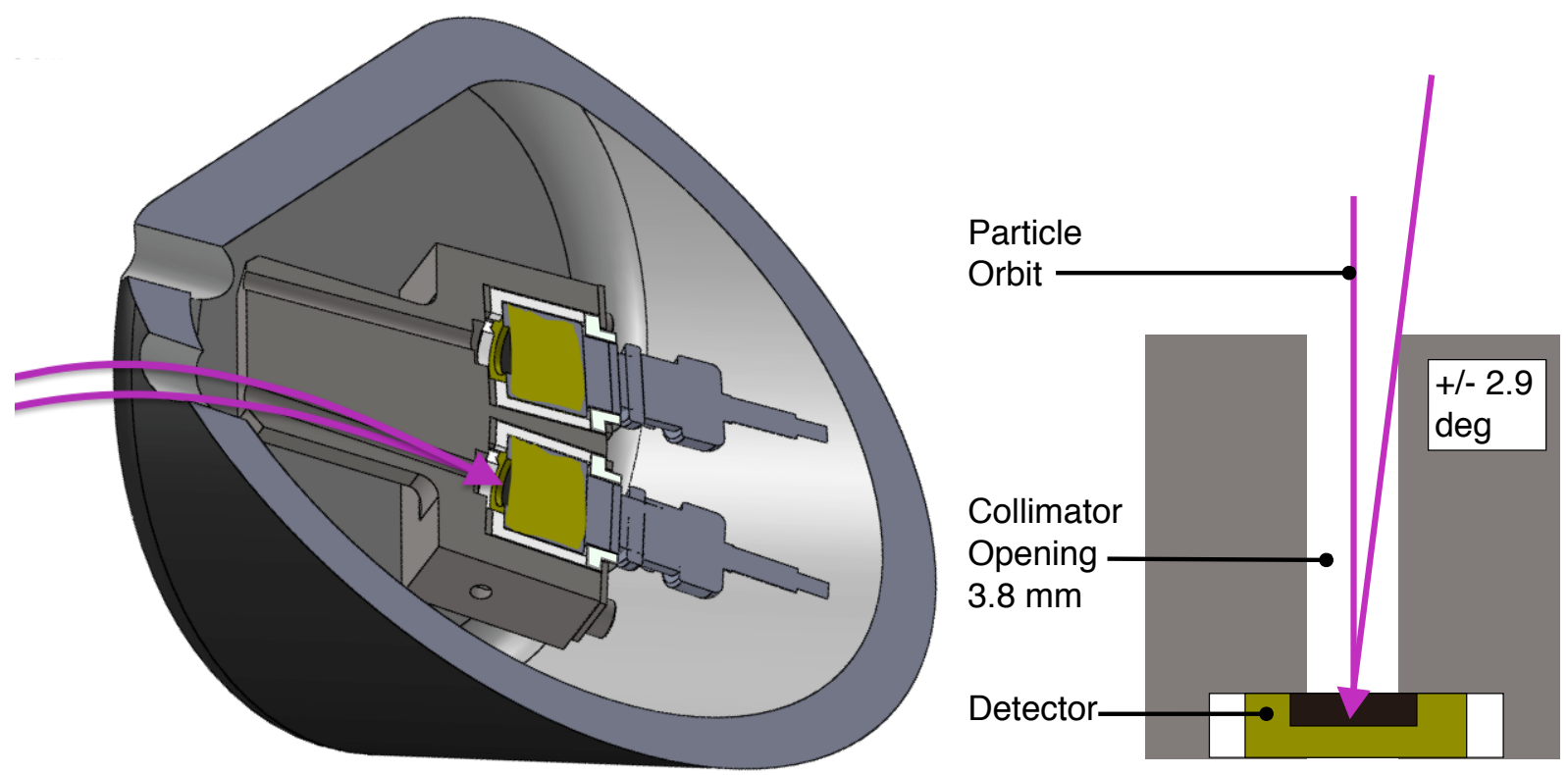

Figure 2.3: The above images are qualitative. A range of trajectories, or orbit bundles, intersect the active area of the detector. This results in a finite thickness associated with each central trajectory, which is characterized by the size of the opening of the collimator for the detector housing.

Calculated Detection Efficiency The overall detection efficiency, $\epsilon$, can be represented by

$$
\epsilon=\epsilon_{I n} \epsilon_{D}
$$

where the intrinsic efficiency, $\epsilon_{I n}$, of a silicon surface barrier detector is assumed to be 1 (or 100\%).[47] The detection efficiency, $\epsilon_{D}$, of the diagnostic calculated by the orbit code can be expressed as the ratio of the number of particles hitting the detector active area to 
the total number of particles created.

$$
\epsilon_{D}=\frac{N_{\text {counts }}}{N_{\text {total }}}
$$

Note that the solid metal collimators in front of the detectors absorb the proton (and triton) energy by a factor of three orders of magnitude quicker than the ions scatter.[47] Therefore, the assumption used in the code is that all detector counts are created by particles emitted from the plasma and that no hits are created by ions scattering off of the collimator walls. Additionally, given the ratio of the collimator length $(38 \mathrm{~mm})$ to the gyro-radii $(0.5$ $\mathrm{m})$, straight orbits from the collimator entrance to the detector active area are used in the orbit code.[47]

The detection efficiency in (2.7) can then be represented by (2.8), $\epsilon_{D P}$.

$$
\epsilon_{D P}=\frac{\int T(\theta) d \theta \int S d l}{2 \pi \iint S d A}
$$

Each segment along the proton orbit is represented by dl. The orbit length is weighted by the emissivity, $\int S d l$, and this value is then weighted by the transmission coefficient, $T(\theta)$, which describes the geometry of the detector's collimator.[48] Additionally, this twodimensional calculation in the poloidal plane assumes poloidal and toroidal symmetry. The typical detection efficiency for a given PD channel's detector-collimator system is on the order of $2 \times 10^{-9}$.

Final Detector Orientations Orbit code particle trajectory simulations were run using different sets of angular orientations for individual detectors (for an array of four detectors). Within a three-dimensional coordinate system, the $\phi$ and $\theta$ angles were chosen for each detector. In general, the angle $\theta$ was chosen such that the detectors look into the tokamak 
and towards the plasma. The set of angular orientations were chosen such that the poloidal projection of the detector channel trajectories intersected the central hottest region of the plasma during different plasma configurations.

Each detector has the same angle $\theta$ so that they all lie in single plane with different $\phi$ angles (see Figure 2.4). Fixing $\theta$ while covering a range in $\phi$ served to make the mechanical housing design simpler. Additionally, this range in $\phi$ is such that regions of the orbits intersecting the plasma mid-plane can span a radial distance of approximately $20 \mathrm{~cm}$.

After running detector configurations for several sets of angular orientations during different plasma magnetic field configurations, the final angles used for the instrument design were: $\theta=40^{\circ}, \phi_{1}=-30^{\circ}, \phi_{2}=-35^{\circ}, \phi_{3}=-40^{\circ}$, and $\phi_{0}=-45^{\circ}$. Note that channel 0 , or detector 0 , corresponds to $\phi_{0}$. Additionally, for these initial simulations a typical radial distance value of $1.8 \mathrm{~m}$ is used to describe the distance from the linear mechanical arm attachment point to the center of the tokamak.

\subsection{General Design Constraints}

There were several design constraints informing the design of a relatively compact instrument for charged particle detection.

Magnetic Properties of Materials The proximity of the diagnostic to the plasma itself required that non-magnetic materials be used for the mechanical probe head. Therefore, stainless steel 316 was used for the main housing. Four pieces of aluminum (Al) were approved for use in the diagnostic because of their thickness of only $0.8 \mu \mathrm{m}$ (each with a surface area of less than $20 \mathrm{~mm}^{2}$ ).

Ultra High Vacuum Environment The protons and tritons cannot travel through the vessel wall as they will deposit their energy into the wall. Therefore the PD had to be installed 


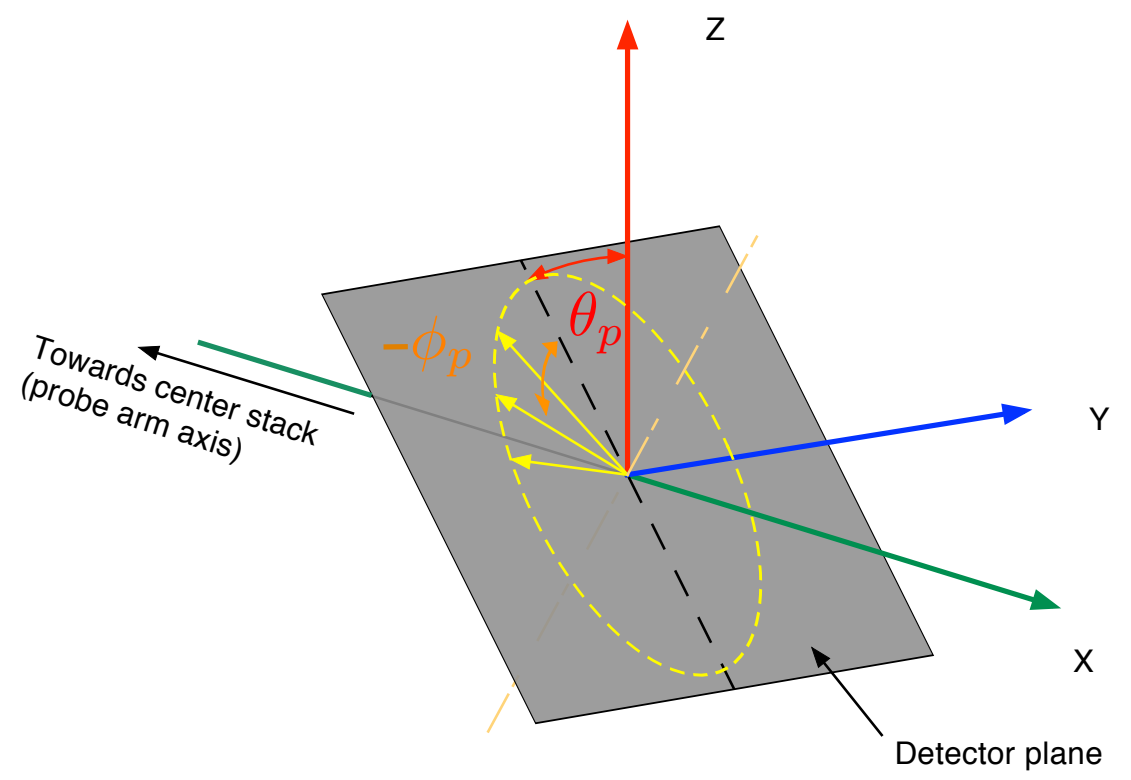

Figure 2.4: Above is the Proton Detector 3D coordinate system. The MAST Reciprocating Probe (RP) arm lies along the x-axis, also called the r-axis. The height above the mid-plane of the tokamak is defined by the z-axis. An angle $\theta$ was chosen such that the detectors looked into the tokamak and therefore towards the plasma. The angle $-\phi$ was chosen such that the detectors looked to the left of the center column of the tokamak. The angle $\theta$, is measured from the $\mathrm{z}$-axis. The angle $\phi$ is measured from the $(-\mathrm{x}, \mathrm{z})$ plane.

inside of the tokamak's UHV environment. An ultra high vacuum installation had two main implications: the assembled mechanical probe head had to be baked at $200^{\circ} \mathrm{C}$ for an extended period of time and the outgassing of the materials could not compromise the UHV environment in which they were installed. For this reason, all materials used were cleaned and approved for UHV installation. Additionally, the detectors and cables were purchased to have UHV approved materials and withstand baking. The CCFE specifically requested the use of Grade-XP Boron Nitride Ceramic for the thermal and electrical shielding of the mechanical probe head. The material was suggested because of its low porosity (it does not absorb much moisture) and high purity, both of which reduce outgassing of the material. Additionally, the CCFE suggested the use of PEEK1000 plastic (PolyetherEtherKetone) 
for insulating electronics within the diagnostic. All coaxial cables used Kapton as the insulating dielectric material and were approved for UHV operation and could withstand baking.

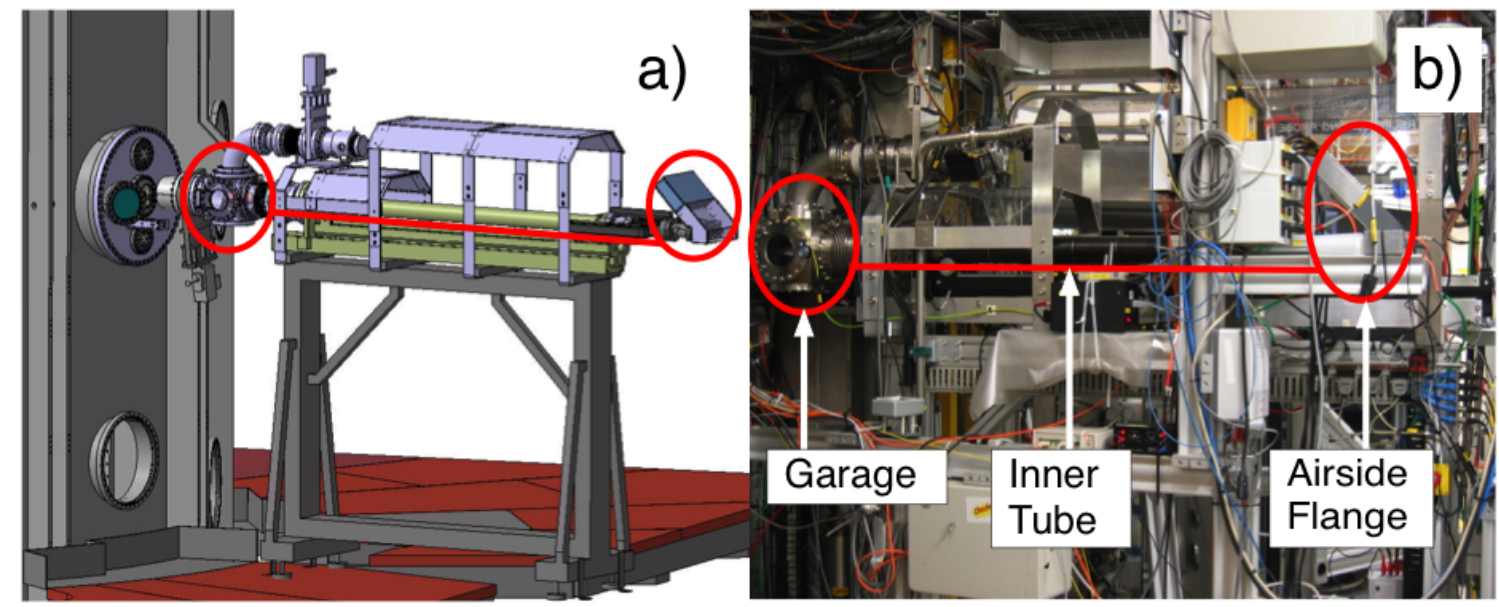

Figure 2.5: Figure a) is a CAD side view of the mechanical arm, or reciprocating probe (RP). Figure $b$ ) is a photo of the RP. The main features of the RP are the garage, the inner tube, and the airside flange. The original CAD image of from the Culham Centre of Fusion Energy.[61][62]

Linear Mechanical Arm The MAST linear mechanical arm used is called the reciprocating probe (RP). The diameter of the assembled mechanical probe head of the instrument had to be less than 12 centimeters $(\mathrm{cm})$; this is because the diameter of the port (located inside of the RP garage) through which the linear mechanical arm pushed the diagnostic is $12.7 \mathrm{~cm}$. The port diameter limited the number and size of the detectors and their corresponding angular orientations in the design.

The assembled diagnostic was physically attached to the end of the RP closest to the plasma. This end of the RP was reached through the garage highlighted in Figure 2.5. The custom connector was designed such that after attachment, the detectors would have the 


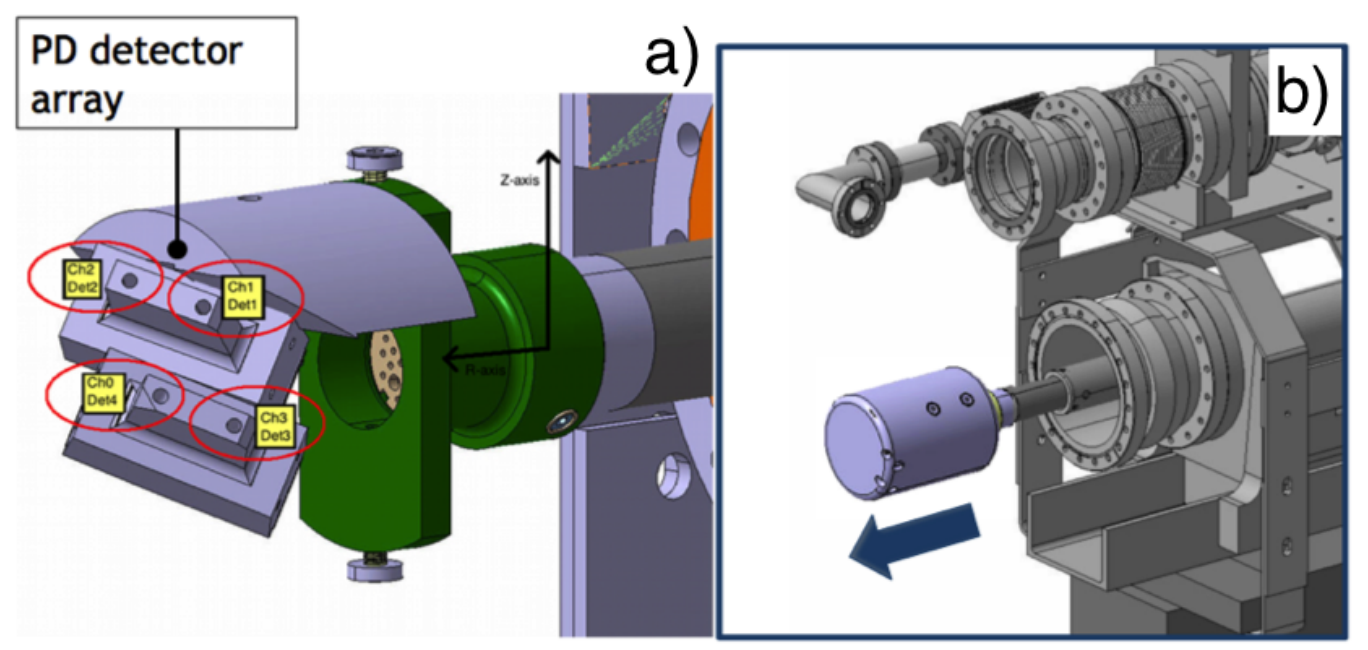

Figure 2.6: Figure a) displays a CAD image of the assembled diagnostic mechanical housing (shown without the protective BN ceramic shield). Each individual collimator opening, for each detector, is circled in red. Figure b) shows the diagnostic physically mounted to the RP. The arrow indicates the direction in which the RP pushed the diagnostic to move it closer to the plasma. The original CAD image of from the Culham Centre of Fusion Energy.[61]

desired angular orientation with respect to the center of the tokamak, see Figure 2.6. The $\mathrm{RP}$ remotely pushed the entire diagnostic towards the plasma or away from the plasma. The remote control of the RP during data collection will be discussed in Chapter 4 .

Remote Data Collection No one is allowed in the vessel while the tokamak is in operation, therefore all electronics for data collection had to be remotely controlled.

\subsection{Mechanical Housing Design}

The PD mechanical housing contains several components. Figure 2.7 displays a CAD drawing of the assembled view of the instrument. The present section provides a brief overview of the components and their assembly (for an example see Figure 2.8). All pieces were individually designed after passing a CCFE mechanical design review. Though the procurement of individual components will not be discussed, a combination of four 


\section{Assembled Diagnostic: $110 \mathrm{~mm}$ Diameter, 185mm Length}
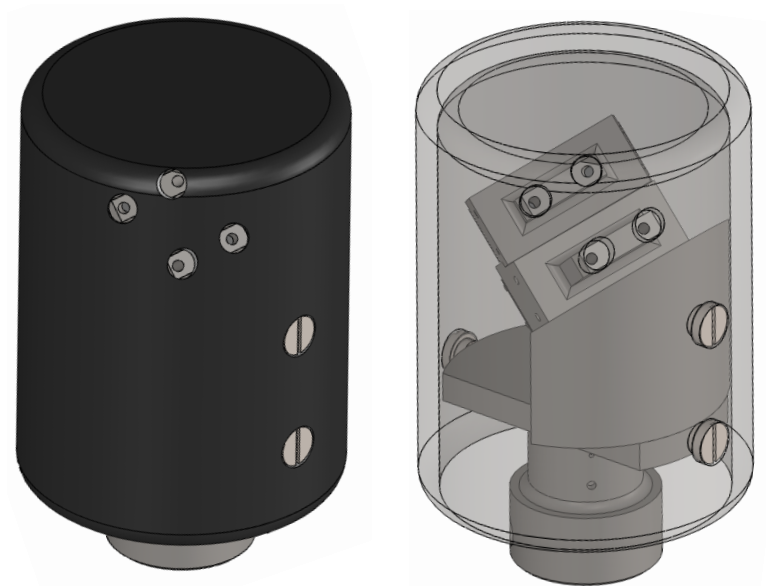

Figure 2.7: The entire assembled PD mechanical housing is displayed in the CAD images above; the shield is transparent in the right image.

machining facilities (external to the research group) were used for manufacturing. The manufactured components were cleaned with a sonicator and cleaning solution and assembled within the group at FIU. Prior to installation, additional cleaning took place at the CCFE. Note that the BN ceramic shield was coated with several layers of graphite. The coating prevented light reflection off the shield which could distort data recorded by nearby instruments. The final assembled instrument was baked in a vacuum oven at $200^{\circ} \mathrm{C}$ for several days. During this initial baking, the UHV environment in the RP arm system was brought to room pressure and opened to allow for installation of the assembled diagnostic after baking. After installation, the RP system was closed and baked before being pumped down to UHV (10-11 Torr) again.

Note that before the installation of the diagnostic and data collection, a prototype instrument (one hollow piece of $\mathrm{Al}$ ) was designed, procured, baked, and installed in the RP system to make sure the dimensions were appropriate. The initial test confirmed the assembled diameter and length to be used for the diagnostic. 


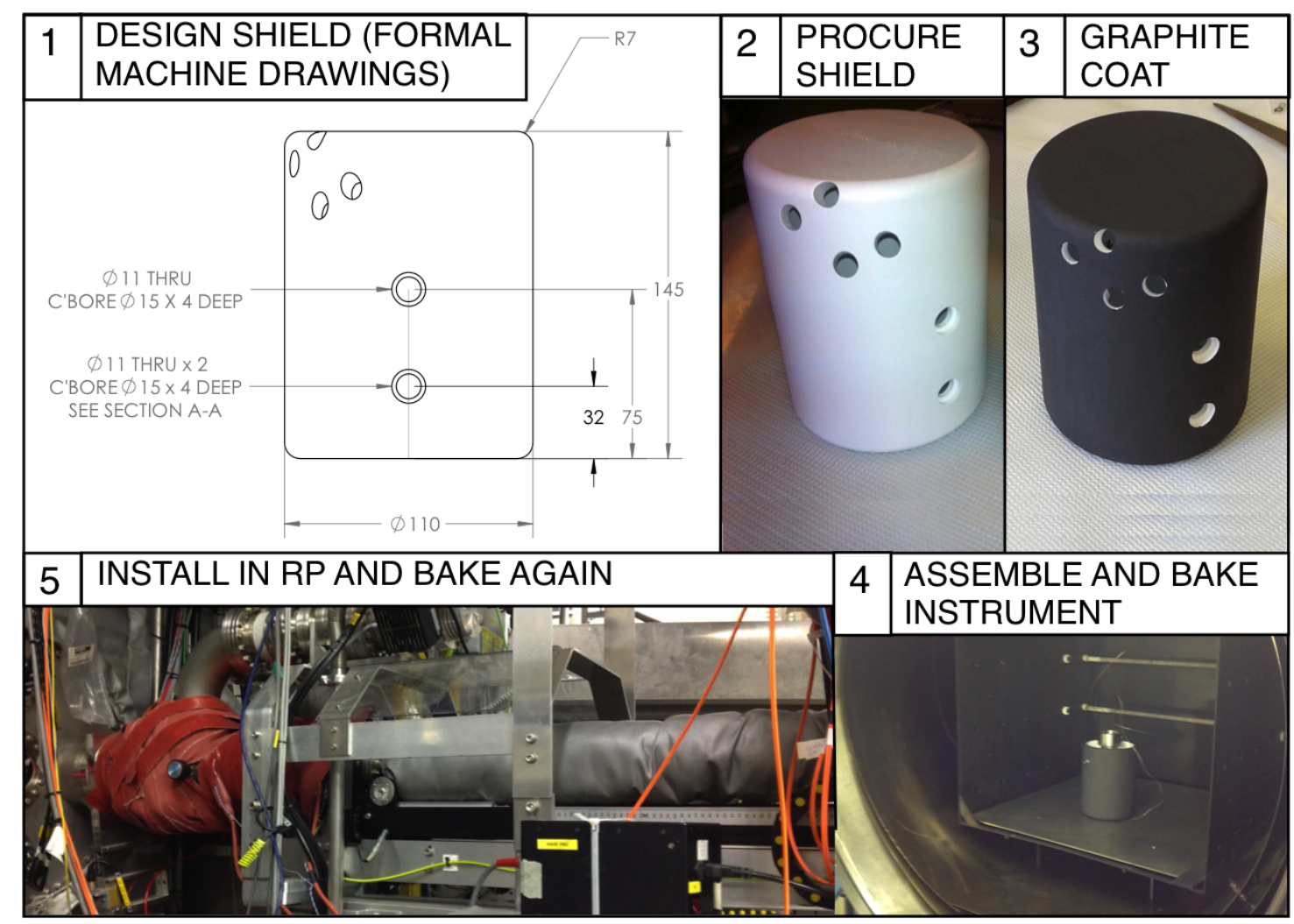

Figure 2.8: The thermal and electrical shield for the instrument was: designed, procured, cleaned, assembled (with other components into one instrument), baked, installed in an UHV environment, and then baked again before its operation for data collection.

Detector Housing The part of the mechanical housing that holds the detectors is called the module, see Figure 2.9 and Figure 2.10. There are two modules; each module holds two detectors. One module contains two sets of: a foil, electrical insulation for the detector, detector, bottom plate to hold the detector, and screws to secure the bottom plate to the module. Note that the detectors are completely insulated from the metal surfaces of the modules in which they are contained, including the bottom plate of the module. The modules determine the detector angular orientations of $\phi_{1}=-30^{\circ}, \phi_{2}=-35^{\circ}, \phi_{3}=-40^{\circ}$, and $\phi_{0}=-45^{\circ}$. 


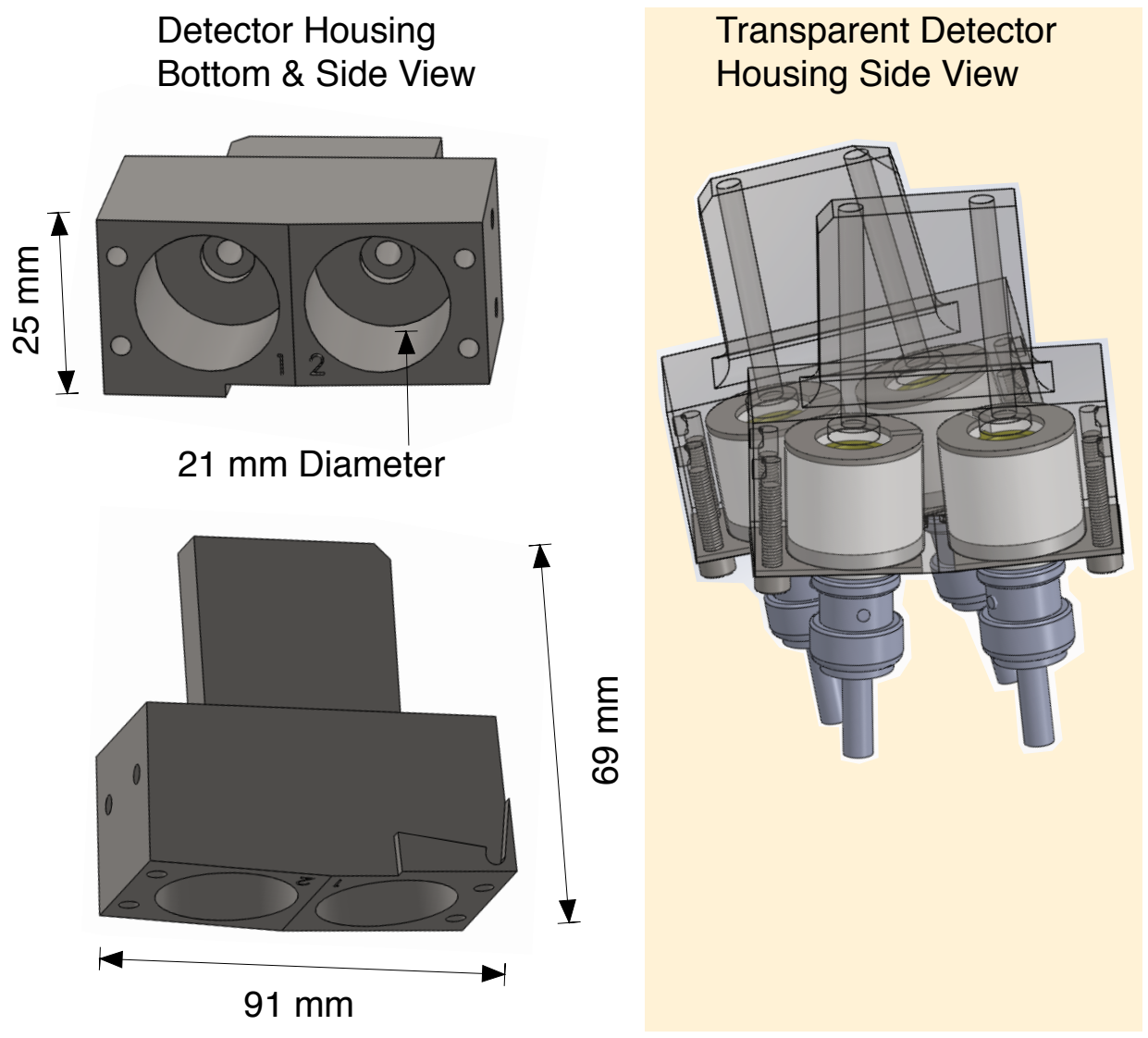

Figure 2.9: Two detectors are housed in each module, as displayed in the CAD images above; there are a total of two modules. The modules ensure the individual $\phi$ angles for detector orientations.

Foil Inside of the module, a metal foil was placed in front of each detector to filter out light and soft x-ray signals. The detector's active layer is $100 \mu \mathrm{m}$. This filter consists of a $0.8 \mu \mathrm{m} \mathrm{Al}$ foil which was spot-welded between two 316 stainless steel washers, see Figure 2.11. The exposed area of the Al foil has a larger diameter than the module's $3.8 \mathrm{~mm}$ collimator opening. Traveling through the foil, protons and tritons lose $18.4 \mathrm{keV}$ and $34.6 \mathrm{keV}$, respectively, through ionization before depositing their energy into a silicon surface barrier detector. When ionization occurs, the incident particles lose energy through Coulomb collisions with the foil atom's electrons. Therefore, the energy lost through ionization is proportional to the electron density of the foil. Additionally, the shorter amount of time the particle spends traveling through the foil the less energy it loses, hence less energy is 


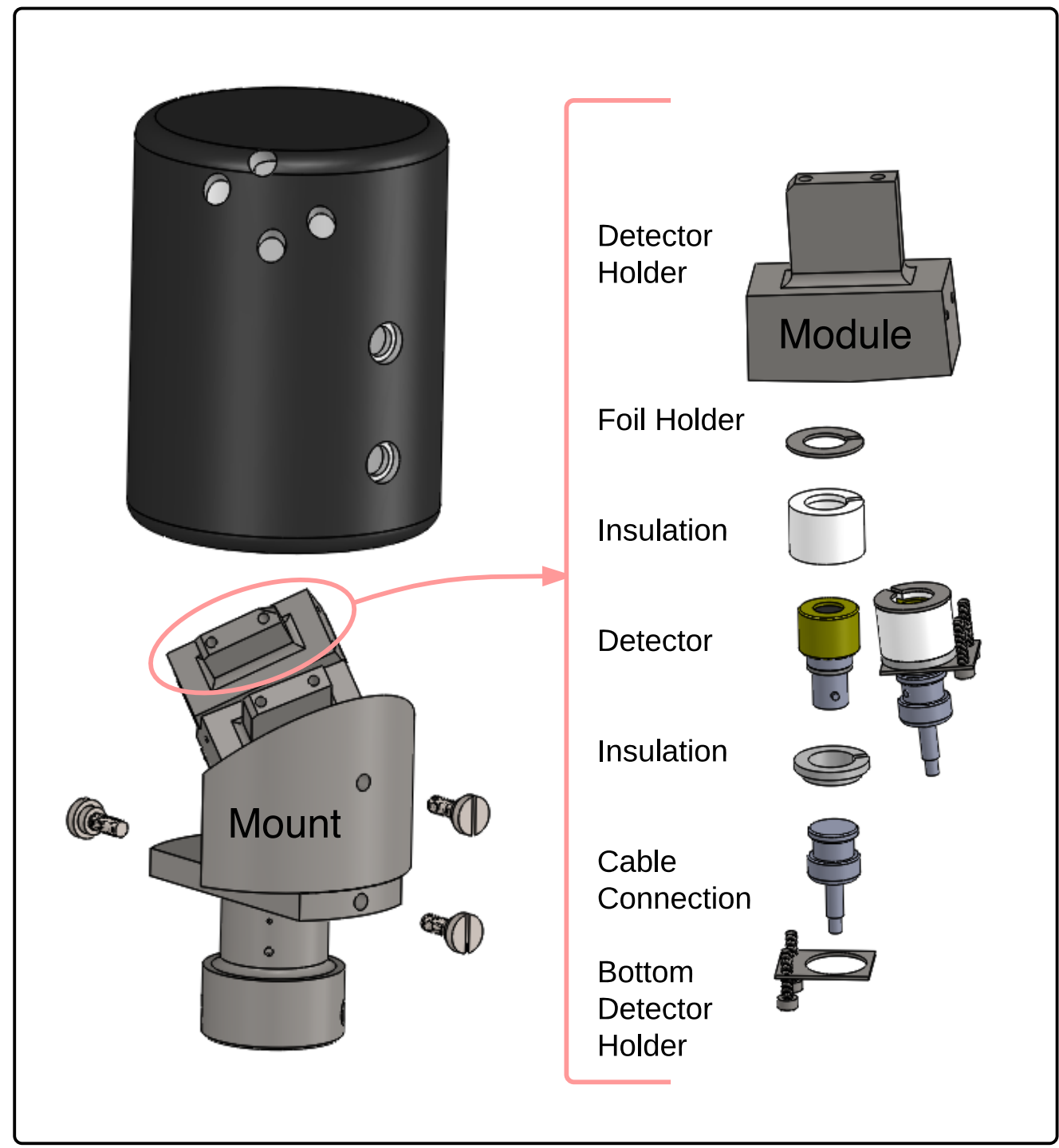

Figure 2.10: The above CAD image shows the housing which holds the detectors, or module. The module contains the: foil holder, electrical insulation for the detector, and bottom plate to hold the detectors.

lost with higher velocities. The estimated energy the particles lose while traveling through the foil was calculated using experimental stopping power (2.9) data from the National Institute of Standards and Technology.[51]

$$
S P=-\frac{d E}{d x}
$$




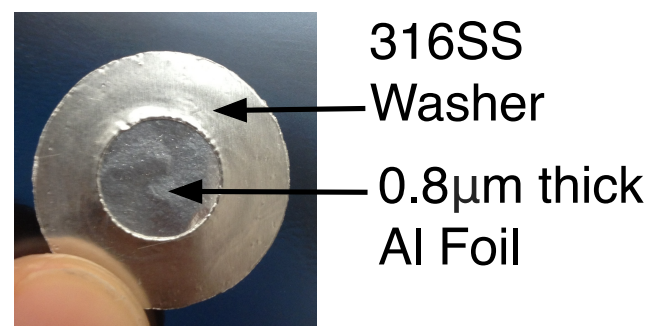

Figure 2.11: A $0.8 \mu \mathrm{m}$ aluminum foil was spot-welded between two 316 stainless steel washers. One foil assembly was placed in front of each detector.

The $S P$ is the particle energy lost through ionization while traveling a distance $x$ in the foil. It can be described by the Bethe equation (2.10).

$$
-\frac{d E}{d x}=\frac{4 \pi e^{4} z^{2}}{m_{e} v^{2}} N Z\left[\ln \left(\frac{2 m_{e} v^{2}}{I}\right)\right]
$$

where the approximation that $v^{2} / c^{2}$ is small ${ }^{1}$ is used.[50] $\mathrm{N}$ is the number of atoms per $\mathrm{cm}^{3}$ in the foil, $\mathrm{Z}$ is the atomic number of the foil, $\mathrm{z}$ is the atomic number of the incident particle, e is the electronic charge, $m_{e}$ is the electron mass, $I$ is the mean ionization potential of the foil, and $\mathrm{E}$ is the energy of the incident particle.

In addition to ionization energy loss, fluctuation in energy loss in the foil is called straggling.[53] Ionization energy loss can cause shifts in peak positions to lower energies in the energy spectrum while straggling can broaden peak widths (full width half maximum values) in the spectrum.[45] Using Bosch's approximation for proton and triton straggling

$$
S T R=12.5 \sqrt{x}
$$

where $\mathrm{x}$ is the thickness (in $\mu \mathrm{m}$ ) of the target foil, a rough estimate of $10 \mathrm{keV}$ would broaden the energy peak width for these two ions.[45] While (2.11) has been used for hydrogen isotopes passing through thin foils, straggling in the foil can have a greater effect on lower energy ions.

\footnotetext{
${ }^{1}$ The $v^{2} / c^{2}$ ratio for protons, tritons, and helium-3 ions are $0.006,0.001$, and 0.005 respectively.
} 
Broadening of the energy peak width can make it difficult to resolve the energy width for lower energy ions.[52] Helium-3 ions already lose $282 \mathrm{keV}$, or $35.3 \%$, of their incident $0.8 \mathrm{MeV}$ energy through ionization. A more general equation to describe straggling was expressed by Bohr as,

$$
\Omega_{B}^{2}=4 \pi Z_{1}^{2} Z_{2}^{2} e^{4} N \Delta x
$$

where $\mathrm{N}$ is the atomic density of the target, $\mathrm{x}$ is the target thickness, e is the electronic charge, $Z_{1}$ is the atomic number of the incident particle, and $Z_{2}$ is the atomic number of the target foil.[53]Theories and experiments further studying straggling apply different correction factors to $\Omega_{B}{ }^{2}$ which depend on the particle incident energy, particle atomic number, and target material and thickness. Therefore, a separate estimate of the energy broadening of helium-3 ions through straggling would be necessary for any future work aiming to resolve these ions' energy spectra along with protons and tritons.

Mounts for Detector Housing The modules housing the detectors were physically attached to a mount, see Figure 2.12. The mount holding the detector modules was then attached to a custom mechanical connector which would attach to the end of the RP which is closest to the plasma. The detector modules, mount, and custom mechanical connector were welded together, see Figure 2.13. The mount determined the angular orientation for all channels to have a $\theta=40^{\circ}$ orientation. 

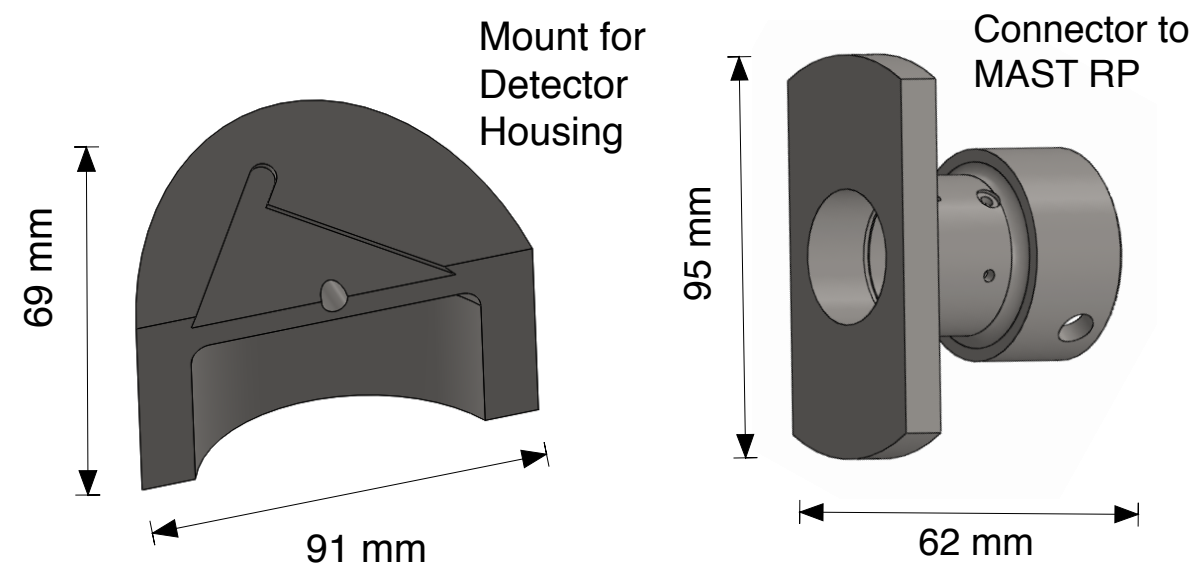

Figure 2.12: The detector modules (displayed in the CAD images above) were physically attached to and supported by a mount and custom connector. The mount ensured the same $\theta$ orientation for all detectors.

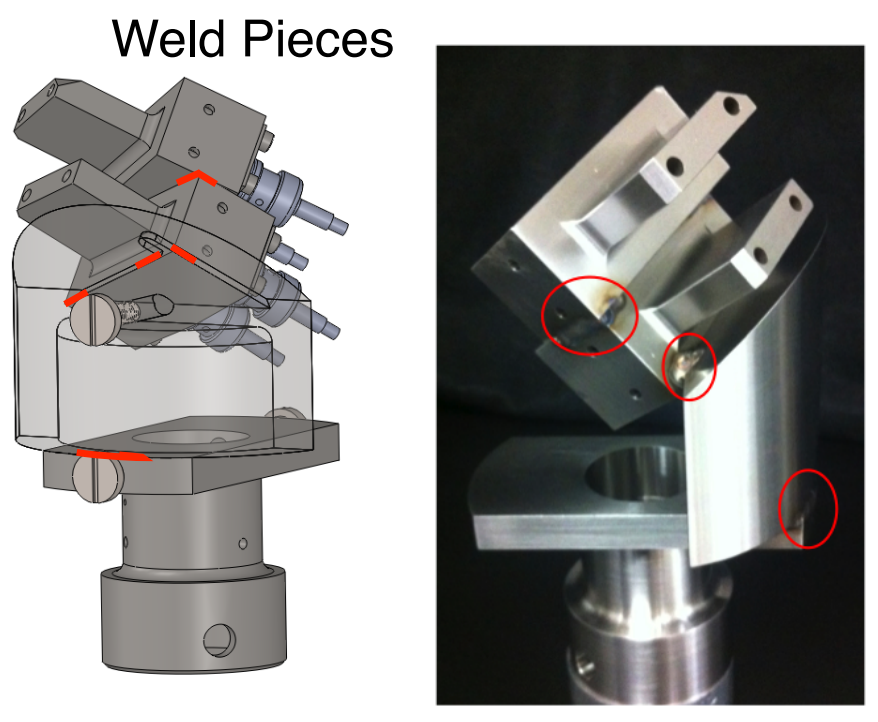

Figure 2.13: The detector modules, mount, and custom mechanical connector were designed such that the detectors had the desired angular orientations when all of the pieces are welded together. The above left image is a CAD drawing. 


\section{CHAPTER 3}

\section{DIAGNOSTIC ELECTRICAL DESIGN}

This chapter concerns the planning and design of the electrical components of the instrument.

\subsection{Data Acquisition System Design}

For a diagrammatic overview of the entire data acquisition system (of four PD channels), see Figure 3.1. Particles emitted from the plasma were detected by a silicon surface barrier detector that also measures its energy. The detector signals passed through the UHV environment along the MAST Reciprocating Probe (RP) $\operatorname{arm}^{1}$. Via a set of vacuum feed throughs, the signals were connected to amplifiers and then collected and stored on a hard drive.

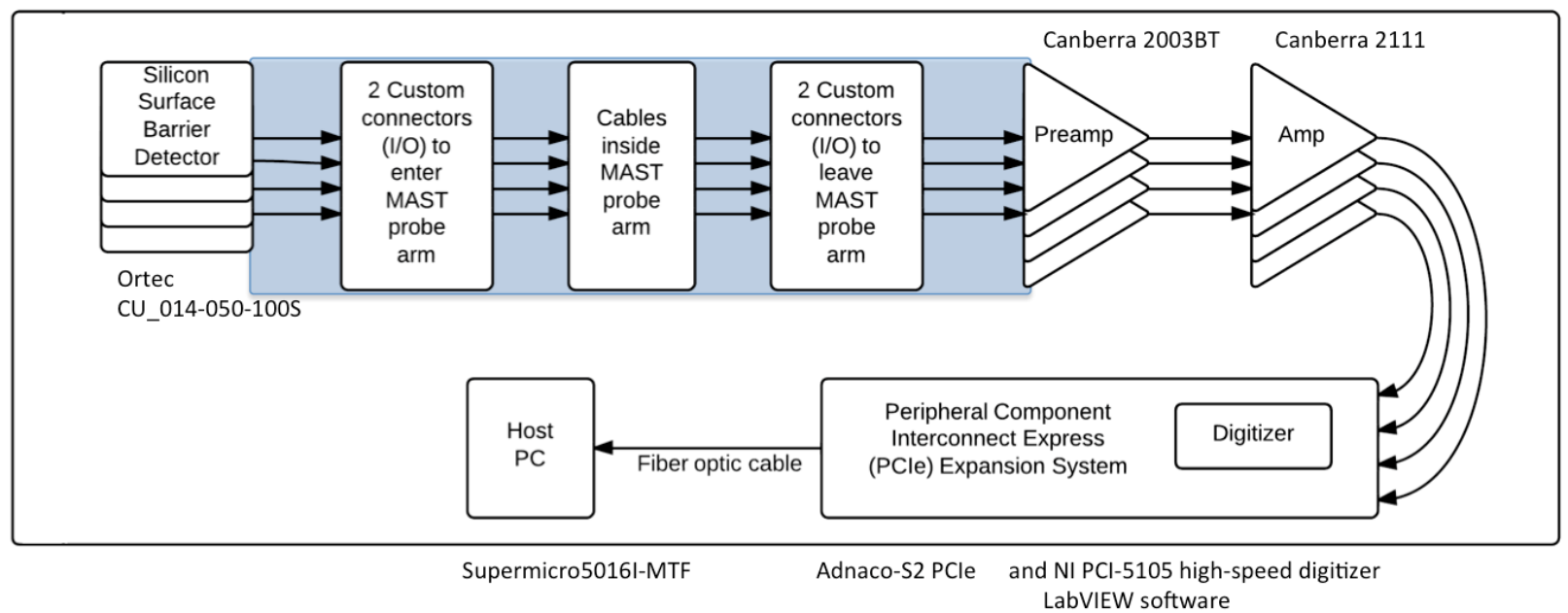

Figure 3.1: Above is a schema for the diagnostic data acquisition. RP installation constraints dictated a distance of approximately $3 \mathrm{~m}$ (blue shaded region) between the detectors and preamplifiers. The distances between items in the schema are not drawn to scale.

\footnotetext{
${ }^{1}$ The RP is a pneumatic linear actuator.
} 


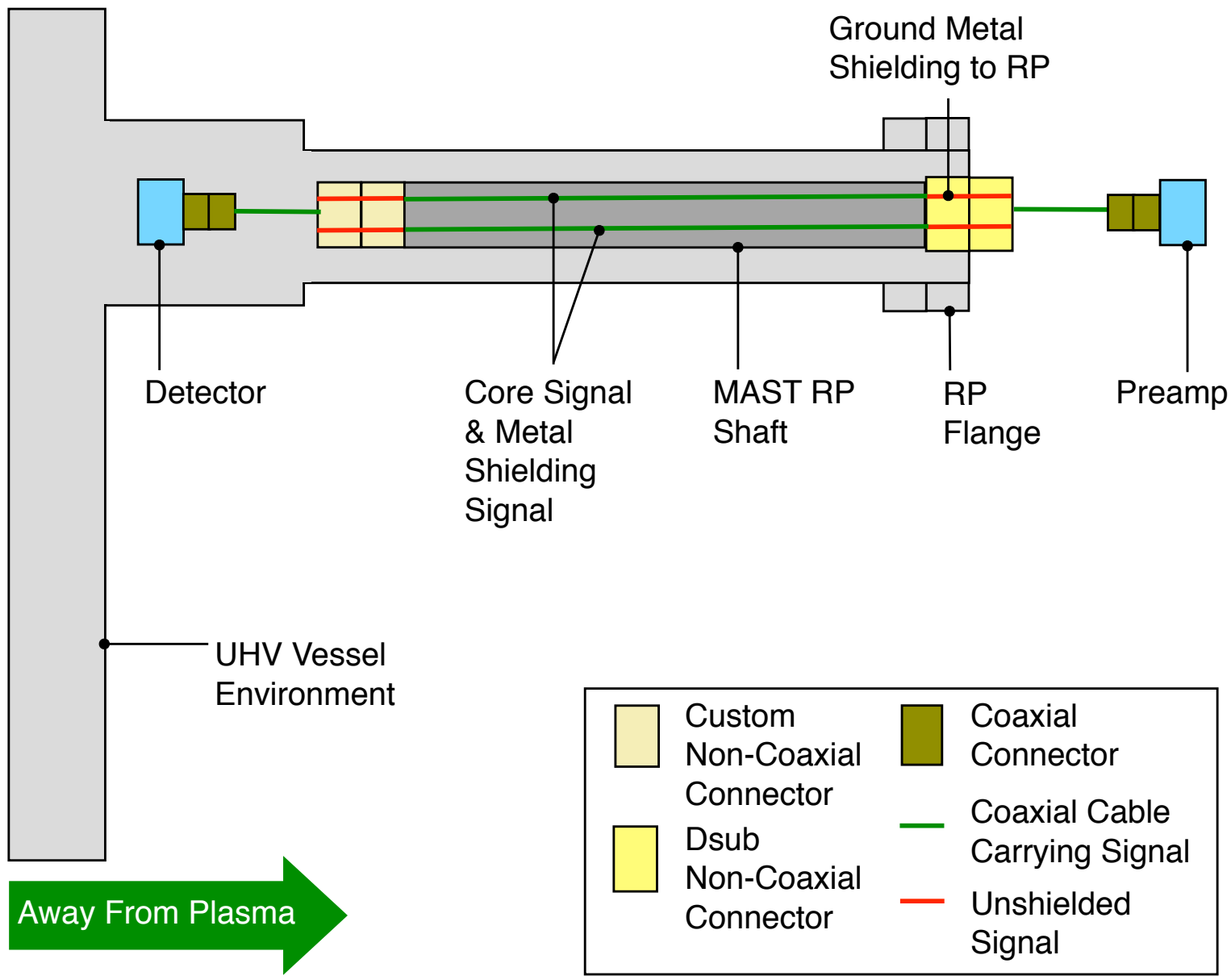

Figure 3.2: The MAST RP had preinstalled UHV approved coaxial cables. A custom non-coaxial connector was installed at the end of the RP closest to the plasma. Similarly, Dsub non-coaxial connectors were required to transfer a signal out of the UHV environment and away from the vessel. An example of one detector signal being transferred through the RP shaft is displayed in the above diagram. The diagram is not drawn to scale.

\subsection{Detector Signals}

After passing through a thin aluminum foil, a particle will be stopped in the active layer $(100 \mu \mathrm{m})$ of the silicon surface barrier detector (Ortec CU014-050100S) thereby creating electron hole pairs which in turn lead to a current pulse. The total charge collected during 
the current pulse is proportional to the energy deposited in the active layer of the detector. An external bias of $+40 \mathrm{~V}$ is applied to the detectors. An external bias applies an electric field across the PN junction in the detector to speed up the charge collection.

The detector signal traveled through two custom UHV non-coaxial connectors to two preinstalled UHV coaxial cables inside of the RP shaft. One signal was for the cable core signal and the other signal was for the metal shielding (or cable screen). Cables were chosen such that there was impedance matching $(50 \Omega)$ between the signal leaving the detector and entering the RP shaft. To make the UHV non-coaxial custom connector, the outer jacket of the coaxial cable, inner braided metal shielding (cable screening), and inner dielectric insulator were cut to expose the inner cable core. The inner cable core and metal shielding were connected to different pins through a crimp attachment. The connector housing is made of PEEK1000 (see Figure 3.3). Note that in the final trial of making the vacuum connector, instead of using a separate wire wrapped around the shielding to connect to the pin, the shielding itself was unwrapped and twisted such that it was directly attached to the pin.

Using a standard UHV coaxial connector for future experiments will mitigate the contribution of noise caused by electromagnetic interference (EMI) since signals would not travel parallel to each other and the cable core signal would remain shielded. Installing a coaxial connector inside of the RP shaft was not available as an option for experiments conducted towards the end of the 2013 MAST operation.

\subsection{Signals Traveling Through the RP}

When the UHV coaxial cable signal entered the RP shaft, the cable core signal traveled along a separate cable which was attached to the cable screening. As the signals from two cables left the RP shaft they joined after traveling through two non-coaxial connectors (15 


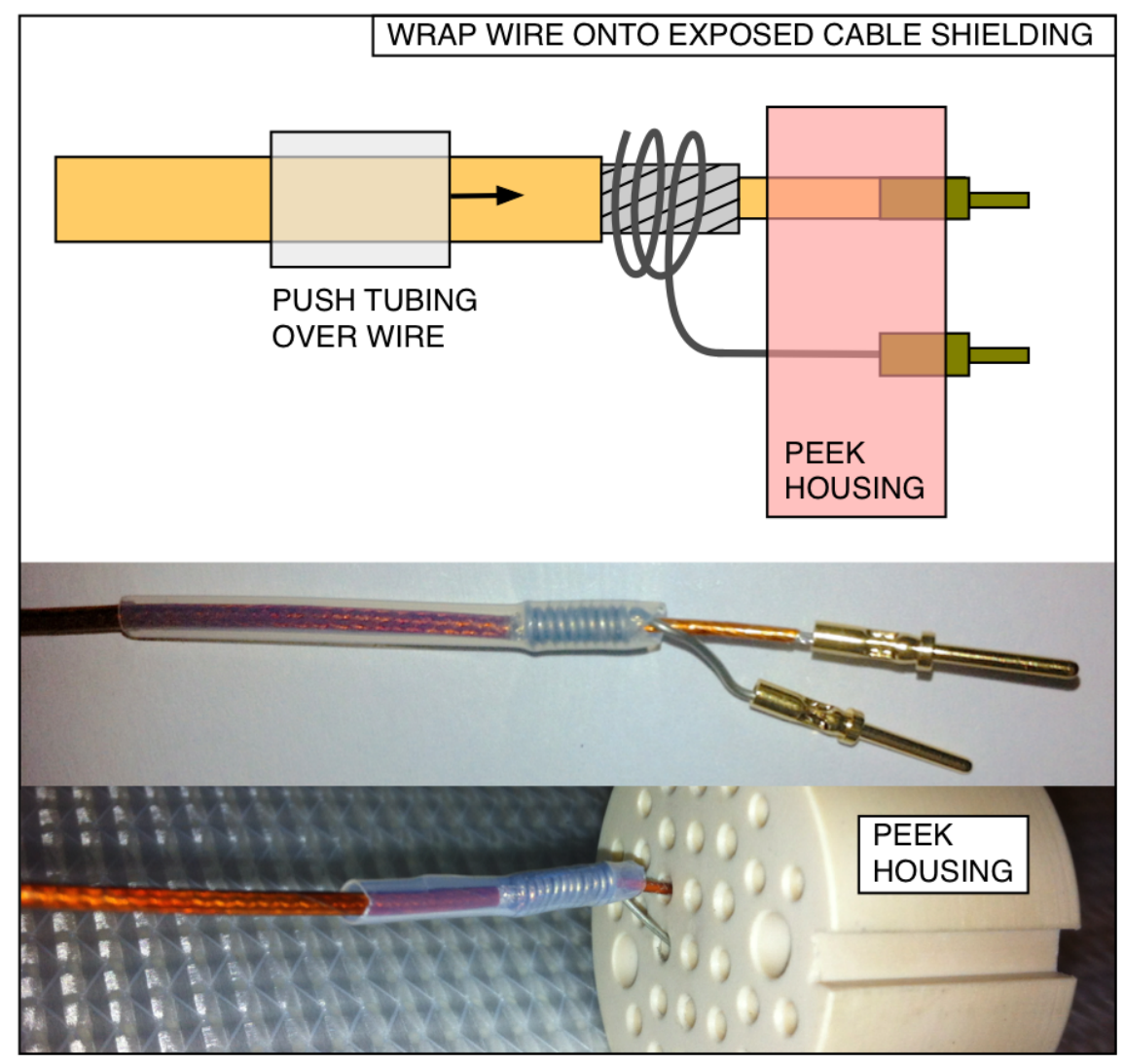

Figure 3.3: The above images (the top image is a CAD drawing) describe the initial method used for attaching the cable screen to a pin. In the final version of the connector, instead of using a separate wire to connect the shielding to the pin, the metal braided shielding itself was unwrapped and twisted so that it was directly attached to the pin (crimp attachment).

pin Dsub) leading to a single airside coaxial cable. Note that the metal shielding (cable screen) signal was grounded to the RP before before traveling through the second Dsub connector. In Figure 3.4, the solid circles in the diagram represent cable core signals and the dashed circles represent the metal shielding signals. The pin numbers are relative to the 15 pin Dsub female connector pin-out. Four signals leaving the detectors are represented by four sets of colors in the diagram.

The initial airside connector connects the signal from the UHV RP shaft to the airside preamplifiers. Note that both signals (for one detector) leaving the RP shaft join into one signal passing through a coaxial cable after passing through the Dsub connector. The pins 


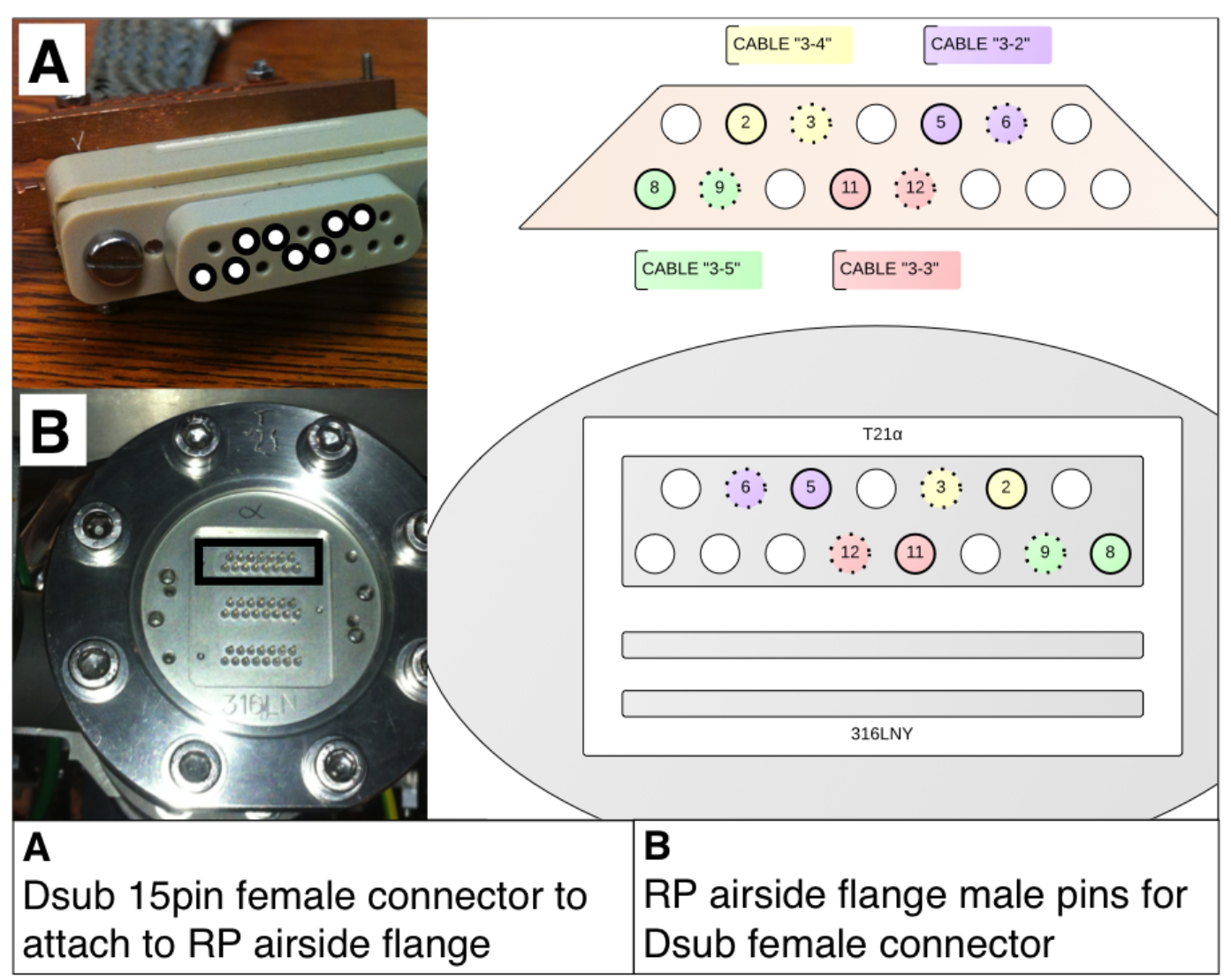

Figure 3.4: The detector signals traveled through the RP airside flange and through a non-coaxial Dsub connector. Afterwards, the signal traveled to preamplifiers mounted next to the RP airside flange. The two images to the right are $\mathrm{CAD}$ drawings with the corresponding pin-out information for the connectors to their left.

are connected to the cable core signal and metal shielding (cable screen) signal using a similar method as described when making the UHV connector. Again, there are a few centimeters of distance in which the signals are traveling alongside each other which can introduce EMI. Figure 3.5 shows the initial airside connector attached to the RP airside flange.

Grounding to the RP before leaving the airside flange caused noise signals within a channel on the order of volts after amplification, which was on the same order of expected particle signals after amplification. This was not observed while testing electronics outside of the RP shaft; only when installing the cables inside of the RP shaft and performing 
the required grounding scheme for the cable screen did this noise appear. Because the grounding of the cable shielding to the RP inside of the shaft was mandatory for installation, other ways of mitigating this noise had to be implemented.

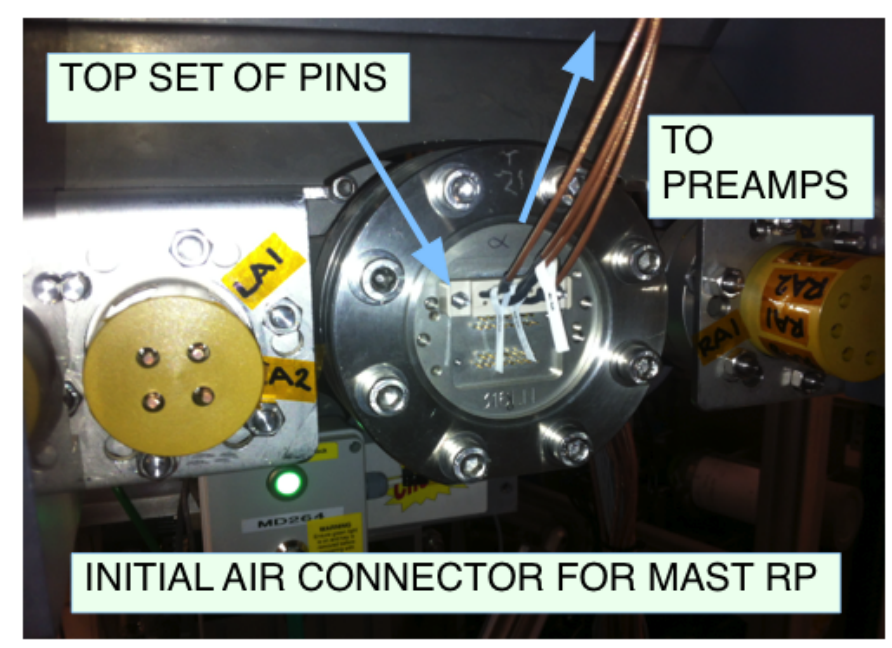

Figure 3.5: This is an image of the first non-coaxial 15 pin Dsub female connector connected to the airside of the RP flange.

During this implementation process, several versions of airside connectors (to transfer the signal from the UHV shaft to the RP airside flange through Dsub connectors) were fabricated. The final 15 pin Dsub female connector was made to lower the background noise in the data channels. Insulation was added to the cable core signal to ensure it did not touch the metal shielding signal. Finally, the cable screen was physically connected to $\mathrm{Sn} / \mathrm{Cu}$ tubular braided sleeving so that the cable screen itself could be grounded to the RP airside structure. The additional grounding at the RP airside structure was implemented to reduce the noise in the data channel to a range between $50 \mathrm{mV}$ and $150 \mathrm{mV}$, see Figure 3.6. Furthermore, a custom copper cover, see Figure 3.7, was made to shield the Dsub connector from EMI. The cover fit over the RP airside flange, had a hole for the cables leading to the preamplifiers, and was connected to the flange with custom clamps (Figure 3.7).

Overall, once leaving the detector the signal traveled approximately $3 \mathrm{~m}$ through the RP shaft and to the airside preamplifier before amplification (the preamplifiers were not 
allowed to be installed inside of the RP shaft's UHV environment). Reducing this distance for future diagnostics through using a UHV-approved preamplifier, will improve the signal to noise ratio as it will amplify signals before they pick up background noise after traveling a considerable distance. Furthermore, using standard coaxial connectors in the vacuum feedthroughs (from UHV to the airside RP flange) for future experiments can mitigate noise.
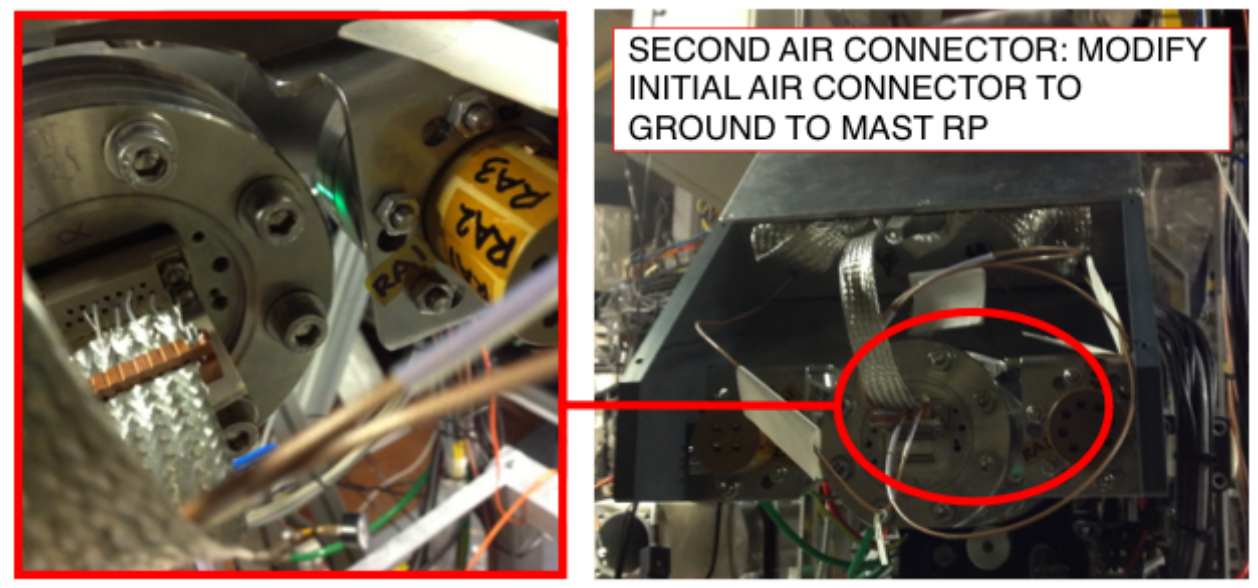

Figure 3.6: This is an image of the final non-coaxial 15 pin Dsub female connector connected to the airside of the RP flange.

\subsection{Amplifying Signals}

After leaving the Dsub connector, the signal reached the airside coaxial cable (LMR195) and traveled to a nearby preamplifier (Canberra 2003BT). A box housing the preamplifiers was physically attached to the RP airside flange structure, see Figure 3.8. Four sets of: preamplifiers, coaxial detector signal cables, preamplifier power cables, and detector bias power cables were stored inside of the housing. In addition to the preamplifiers and input and output cables, braided metal sleeving was attached to provide additional noise reduction, see Figure 3.9. Additional insulating material had been placed between the different 


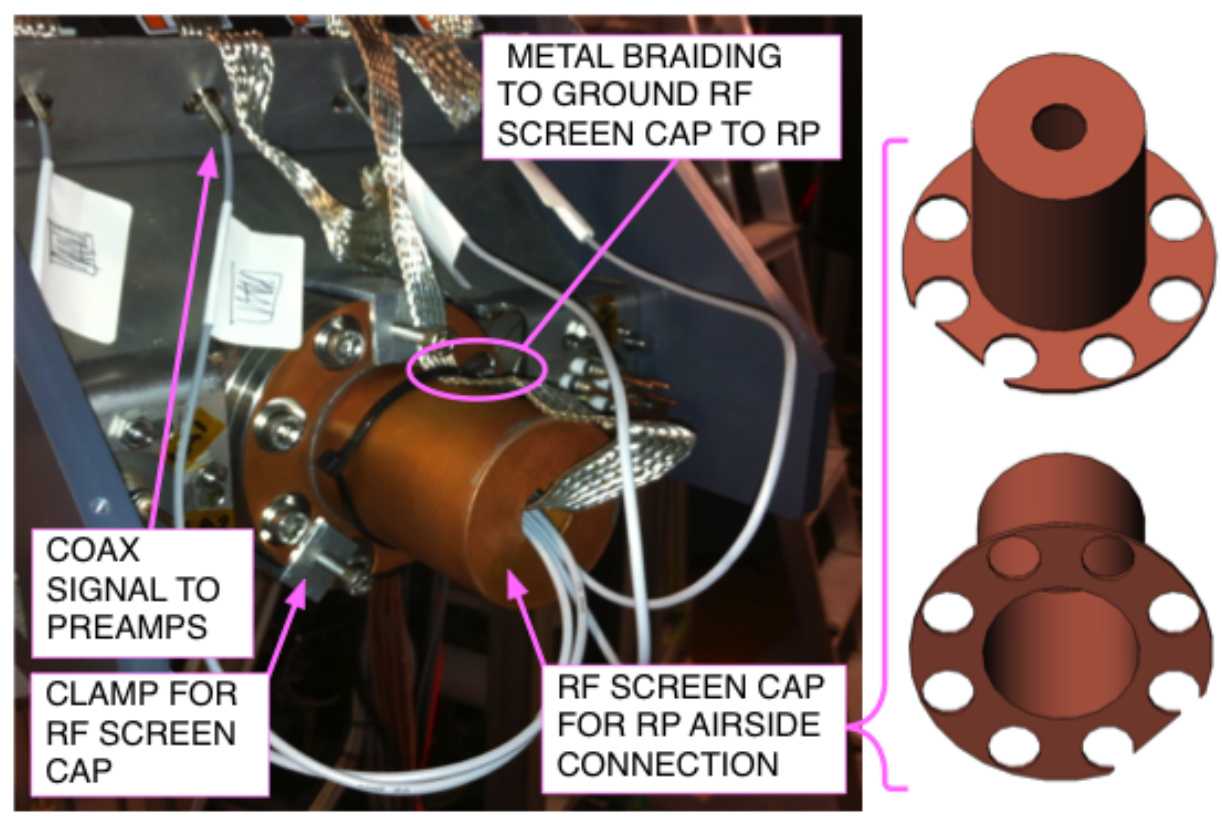

Figure 3.7: A copper cover was made to shield the non-coaxial connector on the outside of the RP flange (leading to the PD preamplifiers) from EMI.

cable connectors to avoid causing ground faults with the surrounding tokamak's vessel. Figure 3.10 displays cabling leading from the RP to the cubicle storing other PD electronics.

\subsection{Data Acquisition Electronics}

The preamplifier signals subsequently passed through a pulse-shaping amplifier (Canberra 2111) and were digitized by a high-speed digitizer (NI PCI5105). Note that the high-speed digitizer was installed in a PCIe expansion system (ADNACO-S2) which connected to a computer (Supermicro 5016I-MTF) through a fiber optic cable. The digitizer was not directly installed in the computer because it could not fit into the computer chassis and the expansion system allowed for the computer to be stored in a separate cabinet farther from the digitizer.

A contributing factor to minimizing noise caused by grounding to the RP included grounding the PD electronics to the RP as well. Figure 3.11 displays the other PD 


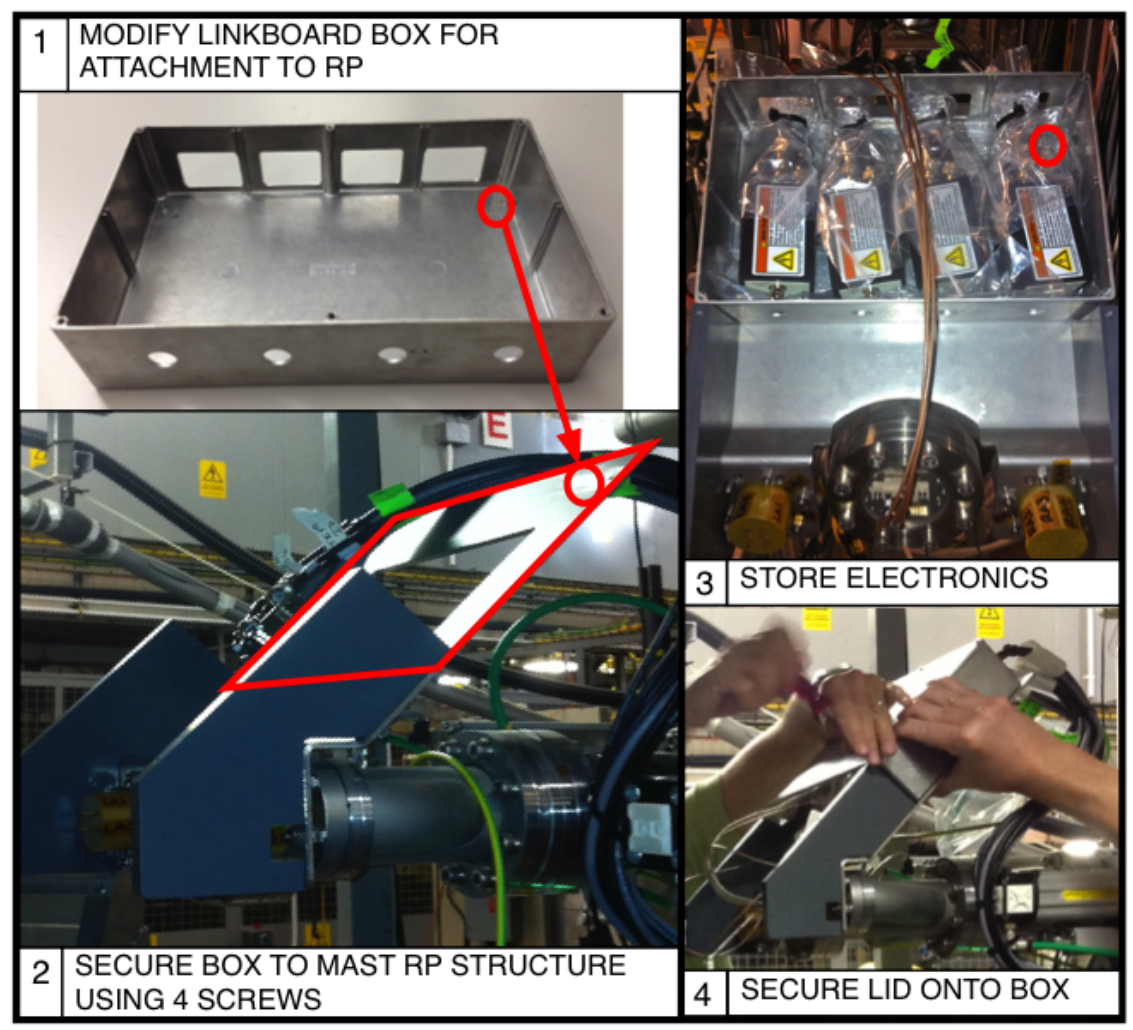

Figure 3.8: A box to hold preamplifiers was physically attached next to the RP airside flange. The box contains four sets of: preamplifiers, coaxial detector signal cables, preamplifier power cables, and detector bias power cables.

electronics stored in the PD01 cubicle: $50 \mathrm{~V}$ power supply (A), digitizer (B), CAMAC crate (C), amplifiers (D), and trigger input signal (E). The new cubicle was positioned next to the RP airside flange so that it could be grounded to the RP structure. The PD01 cubicle was insulated from the vessel floor through sitting on blocks of insulating material (Figure 3.14). A CAMAC crate was used instead of a NIM BIN so that a trigger could be sent to the system through a fiber optic cable. Additionally, the computer could be left in another cubicle because it was connected to the PCIe expansion system through a fiber optic cable.

As seen in Figure 3.12, a custom power distribution unit (PDU) was made for the PD01 cubicle. The PDU contained fused spurs, one for each piece of equipment (power supply, PCIe expansion system, and CAMAC crate) that needed to be provided with mains power. 


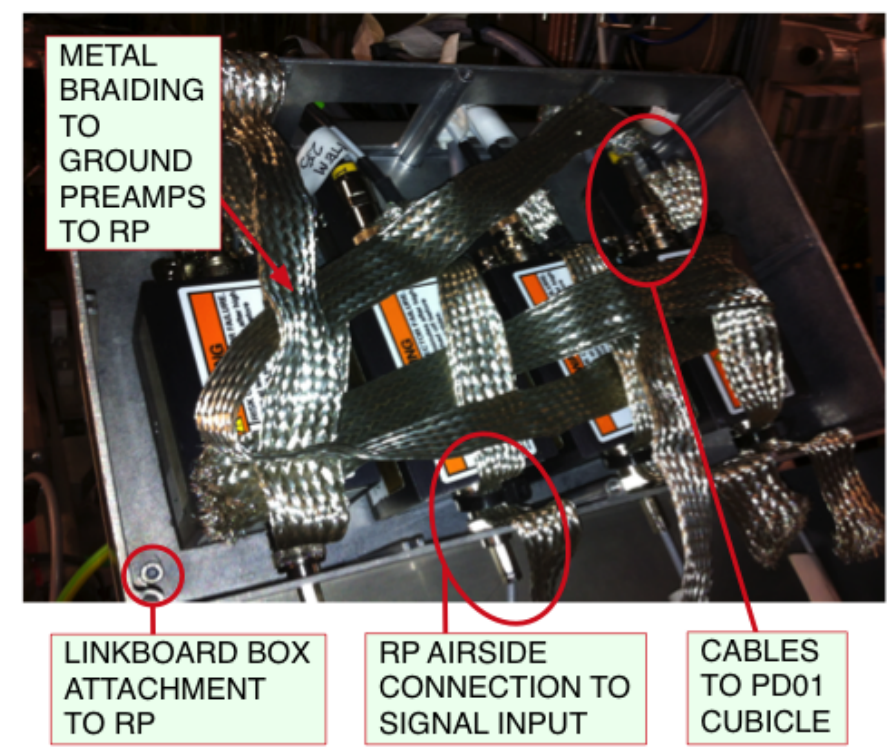

Figure 3.9: In addition to the preamplifiers and input and output cables, braided metal shielding was attached to provide further noise reduction in the signal.

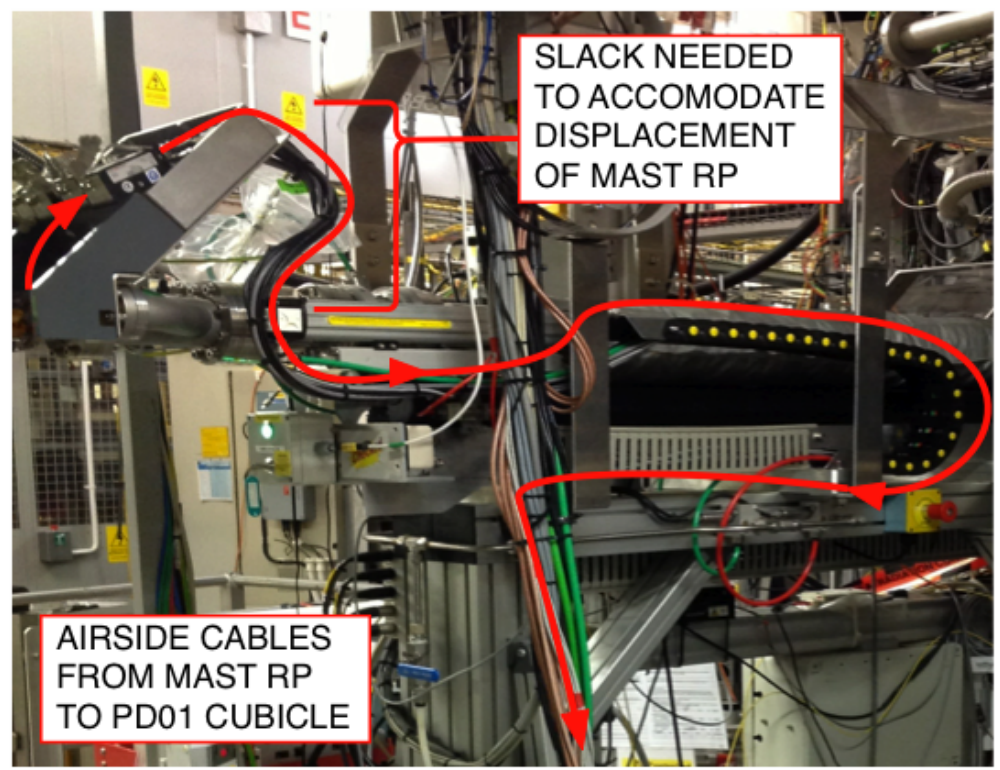

Figure 3.10: The output signal cables from the PD preamplifiers were placed in a cable tray that led towards a cubicle containing other electronics. Slack was needed in the cables to accommodate the RP movement towards and away from the tokamak.

The PDU drew its mains power from the MD62 cubicle, which ensured that the PD electronics were grounded to the same point as the RP. The MD62 cubicle contained the power source and final grounding point for the RP. 


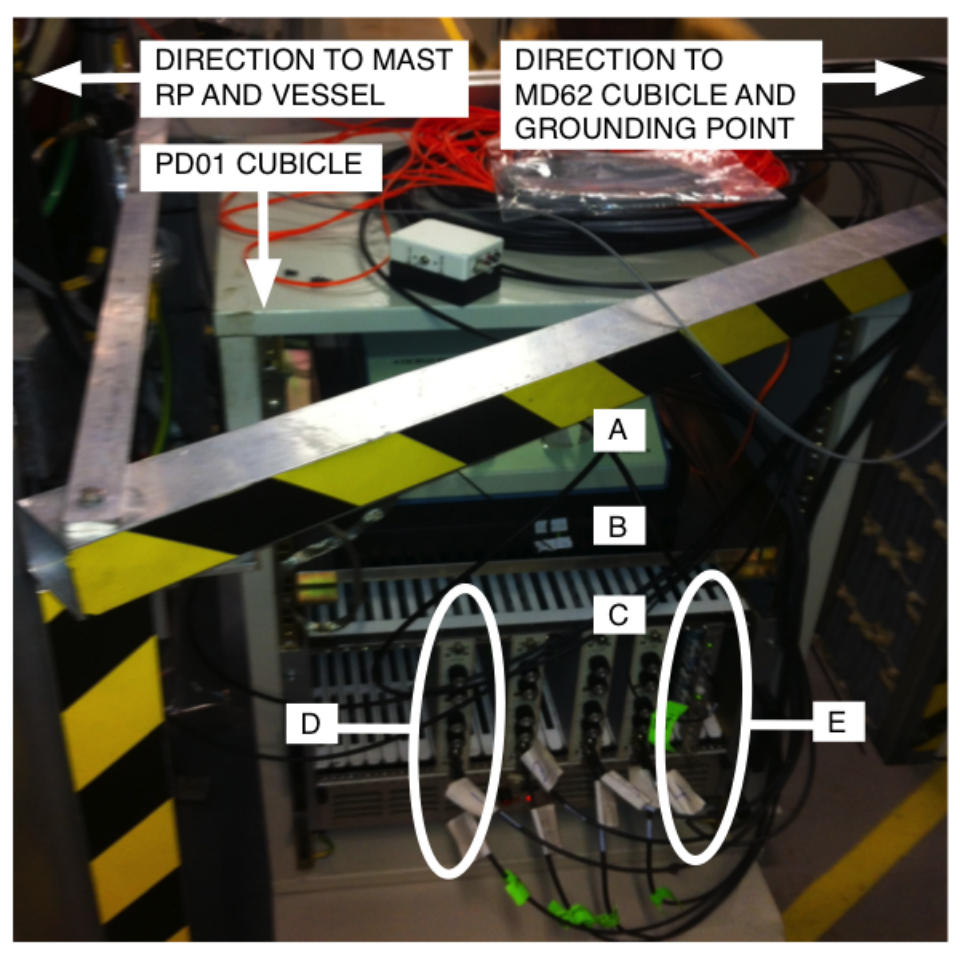

Figure 3.11: To reduce noise caused by grounding the detector signals to the $\mathrm{RP}$, the original cubicle was changed to the PD01 cubicle. The electronics: $50 \mathrm{~V}$ power supply (A), digitizer (B), CAMAC crate (C), amplifiers (D), and trigger input signal (E) were stored in the PD01 cubicle. This image shows the front of the cubicle.

Before grounding the PD01 cubicle itself to the RP grounding point, the walls of the cubicle were electrically bonded to each other, see Figure 3.13. A summary of the electronics and PD01 cubicle grounding to the RP structure and final grounding points can be found in Figure 3.15. The cubicle was physically grounded to the RP as well as through its custom PDU. The electronics (labeled 1, 2, and 3 in Figure 3.15) were physically grounded to the cubicle and grounded to the RP grounding point through the custom PDU. The grounding scheme was necessary to reduce noise levels from several volts to hundreds of millivolts after amplification. 


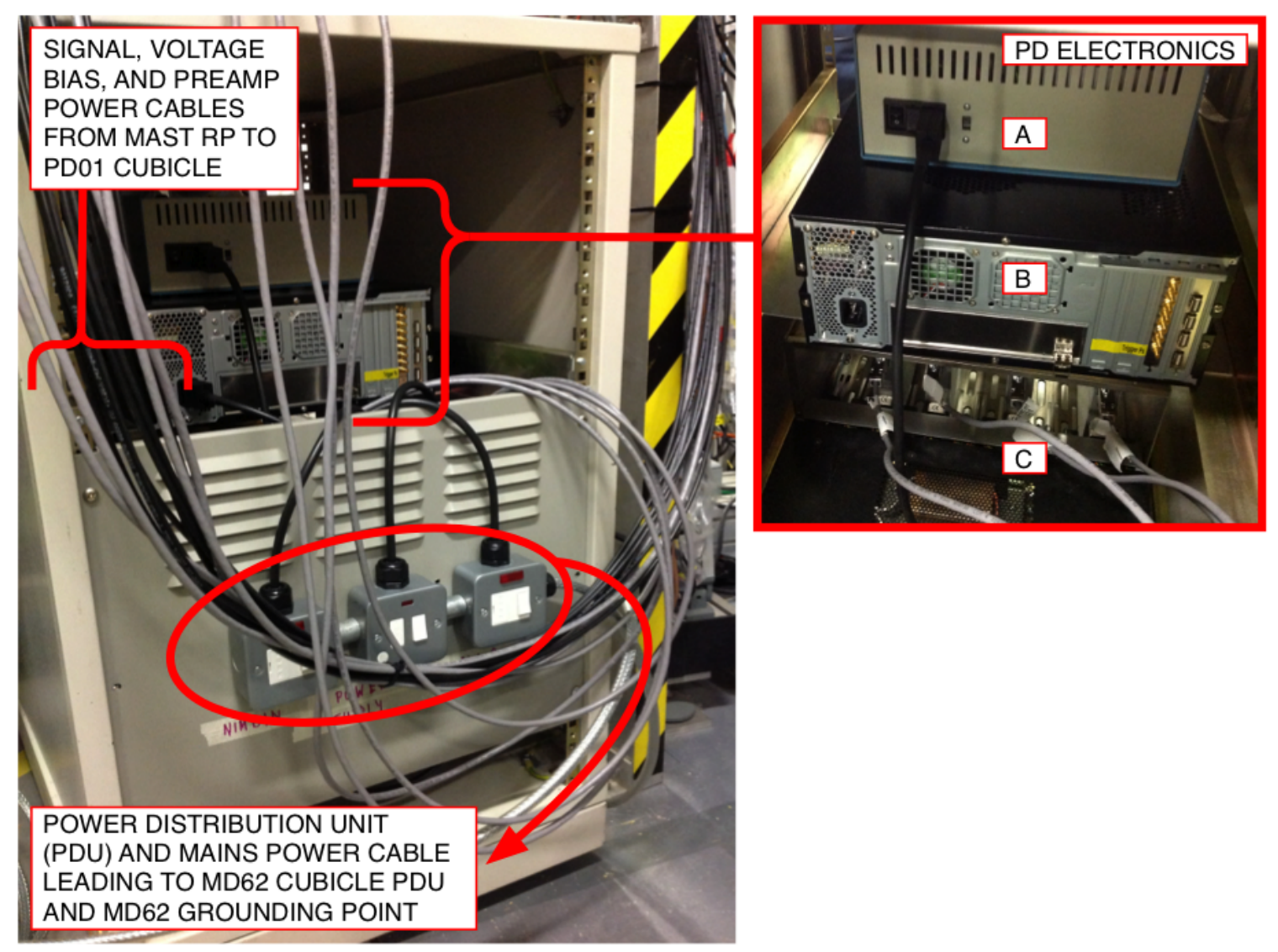

Figure 3.12: The electronics: $50 \mathrm{~V}$ power supply (A), digitizer (B), and CAMAC crate (C) were stored in the PD01 cubicle. This image shows the back of the PD01 cubicle. A custom power distribution unit containing fused spurs was made for the cubicle. The power for the unit led to the grounding point and power source for the RP. All electronics had the same grounding as the RP.

\subsection{Data Acquisition Software}

The data acquisition program was written in LabVIEW to save the digitized signals and to automate data collection. The first section of the code (for the program) configures and activates the appropriate digitizer channels for data collection. The second section of the code configures the digitizer to wait for a trigger. The third section of the code configures the digitizer to save data once it receives a trigger signal. The last section writes data files and returns any error messages. Depending on the mode of operation, the program 


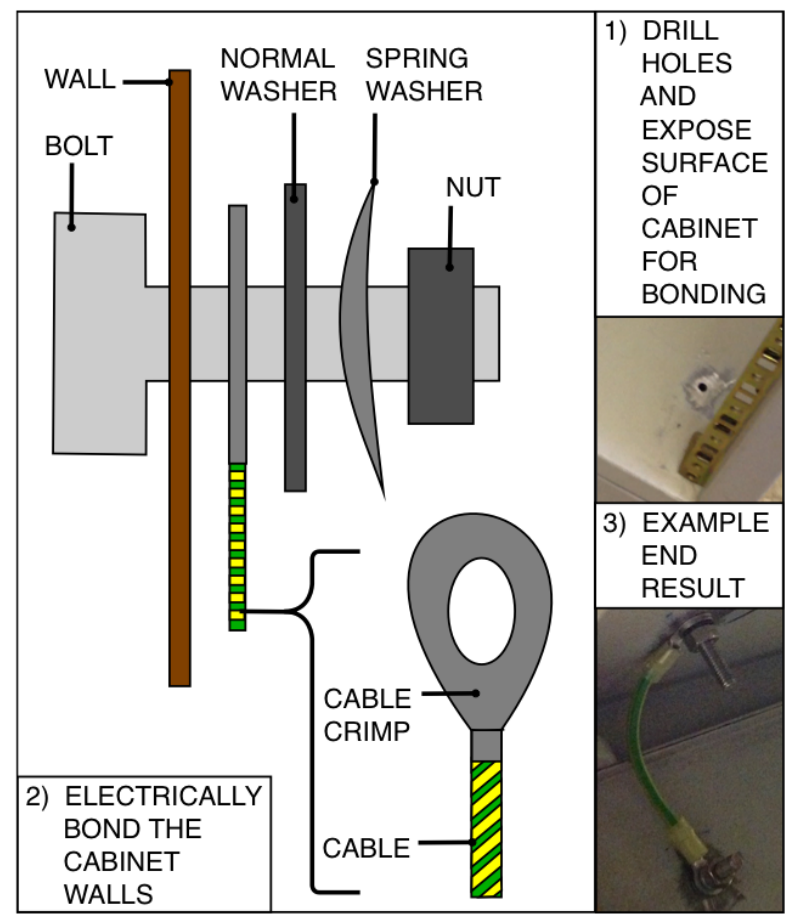

Figure 3.13: The walls of the PD01 cubicle were electrically bonded to each other.

will either end or loop to the second section of the code to automatically wait for the next trigger. See Figure 3.16 to view several user input parameters to specify operational settings for the data collection.

Digitizer Parameters Digitizer input channels that needed to be enabled for data collected were specified as well as the desired digitizer sampling rate $(\mathrm{Hz})$. Note that the maximum sampling rate is $60 \mathrm{MHz}$. With $512 \mathrm{MB}$ of onboard memory, if data are simultaneously collected for four channels the maximum amount of time for which data can be saved is 0.65 seconds $^{1}$, where $128 \mathrm{MB}$ of memory per channel was used. Saving data for 0.65 seconds was sufficient for plasma experiments lasting 0.5 seconds. The appropriate voltage range for expected signals was another input parameter to the code. The digitizer has a maximum signal input of $10 \mathrm{~V}$.

\footnotetext{
${ }^{1}$ This is not a hardware limit but a limit resulting from the computer operating system and efficiency of the written program.
} 


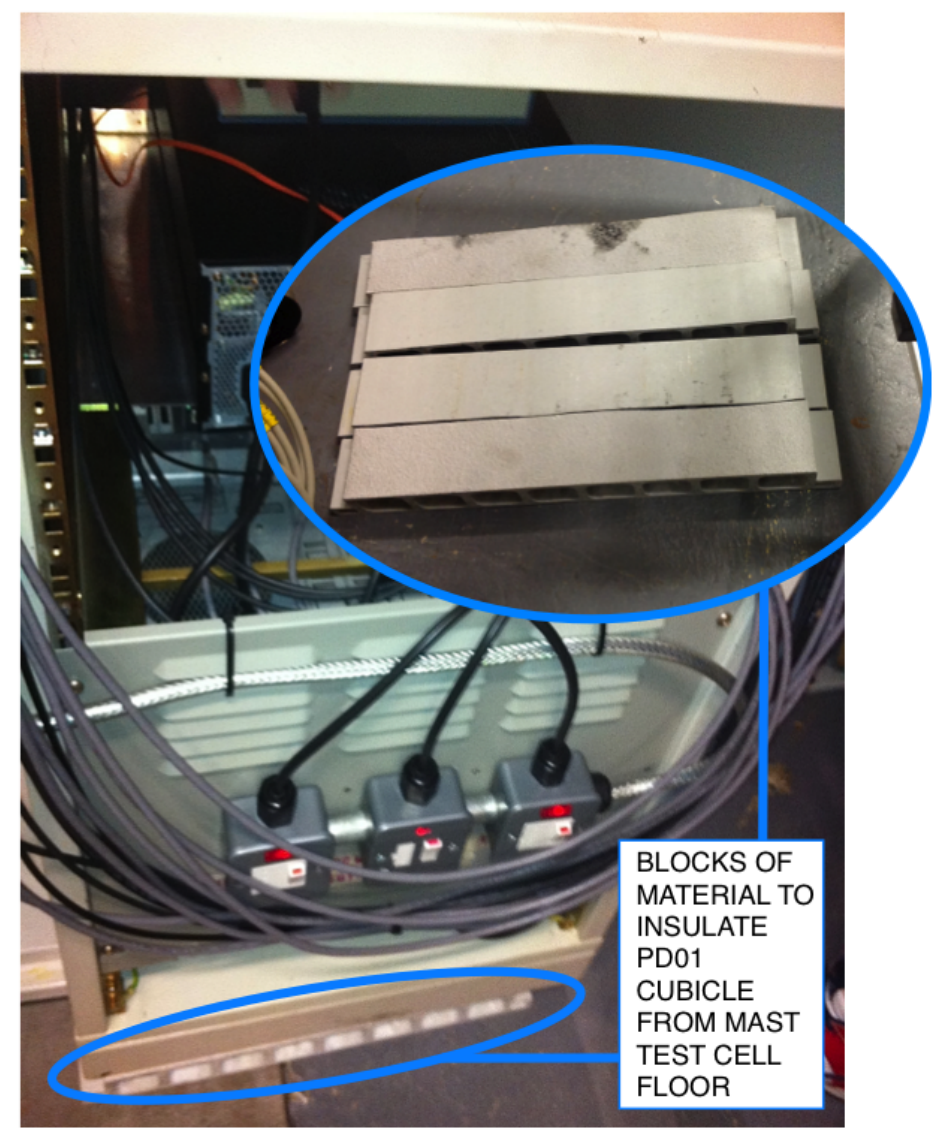

Figure 3.14: The PD01 cubicle was insulated from the vessel floor through sitting on blocks of insulating material (fiber glass).

Some digitizer parameters do not appear in the program windows, they are programmed into the background code so as not be changed by the user. An impedance of $50 \Omega$ and DC coupling were used for all input signals because of the cables and accompanying electronics used into the data acquisition system design.

Trigger Parameters The timing parameters for data collection were an important factor to relate collected PD data to MAST discharges as well as data collected with other instruments.

A $5 \mathrm{~V}$ digital signal (lasting $0.5 \mathrm{~s}$ ) was used as a trigger input signal. When the rising edge of an external trigger signal is received the digitizer starts to record voltage signals 


\section{RP GROUNDING SCHEME}

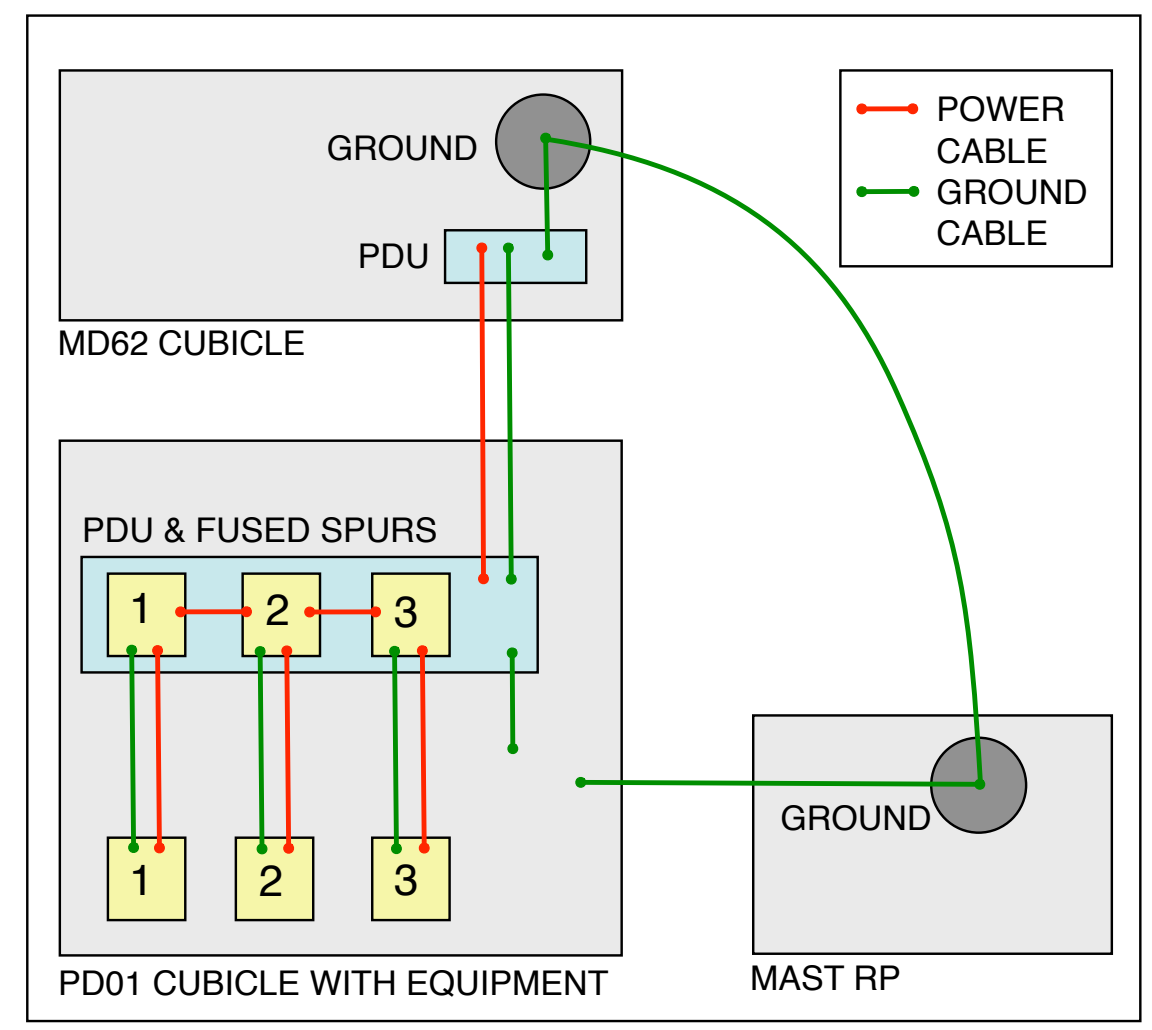

Figure 3.15: The RP structure was grounded to the MD62 cubicle grounding point. The PD01 cubicle was grounded to the RP structure grounding point as well as to the MD62 cubicle grounding point (through the fused spurs and power distribution unit). The PD electronics (labeled 1,2, and 3) were powered through the PD01 cubicle power distribution unit.

for the duration of the requested data collection time. The time the digitizer waits between receiving the trigger signal and saving data can be changed through user input parameters. The channel receiving the trigger signal is different from the channels used for data collection. Similar to the digitizer parameters, the appropriate voltage range needs to be selected for the size of the trigger signal. In addition to this range, a minimum threshold for the trigger signal is also specified as a user input. Another important parameter to set, especially for the default program mode of operation, is the trigger timeout value. The trigger 


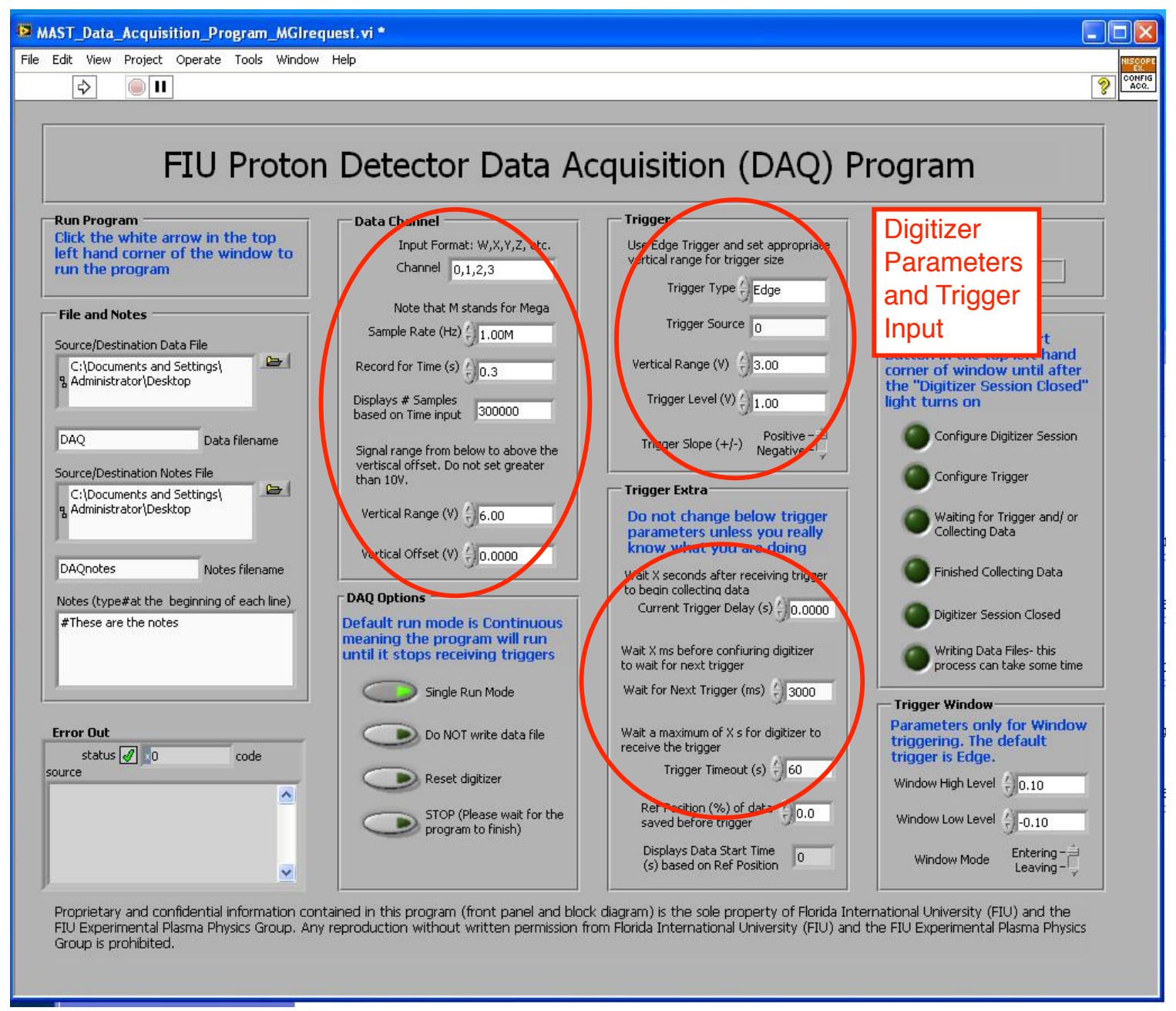

Figure 3.16: Custom LabVIEW code was written to remotely collect data at MAST.

timeout is the maximum amount of time the digitizer will wait for the trigger until exiting the program with an error. The amount of time can be set for seconds, minutes, or hours depending on the logistics for data collection.

For example, during data collection (described in Chapter 4), the MAST trigger signal was sent $10 \mathrm{~ms}$ before the plasma discharge. The program told the digitizer to trigger on the rising edge of the signal and then wait $70 \mathrm{~ms}$ after receiving the trigger to start collecting data. This means that the program started saving data $60 \mathrm{~ms}$ after the plasma discharge began; this was appropriate because it generally takes over $100 \mathrm{~ms}$ for the NBI heating (the 
time of interest) to begin. The trigger timeout was usually set between six to eight hours so that the program would receive triggers and record data throughout the entire day without user interaction.

Program Mode Options When no special modes are selected, the default mode of operation for the program is to continuously run as long as trigger signals are received. Depending on the timeout parameter chosen, the program can run for an entire day. This default mode of operation accommodated MAST experiments.

The program has several modes of operation that can be selected while troubleshooting or testing the software. The Single Run Mode option means that after writing and saving a single data file (after receiving a single trigger), the program will end. The Do Not Write Data File Mode operates the same as the default mode except it does not write data files. The Reset Digitizer Mode is an option to reset the digitizer; this is necessary if the program is forcibly closed before the digitizer receives a trigger for which it is waiting. The STOP mode means that the program will end after receiving the next trigger.

Data Storage Folders to where data files (and relevant notes) will be stored are specified before running the program. Each saved file will have the timestamp (at the time the file is written) incorporated into the file title. Note that each channel stores around $30 \mathrm{MB}$ of data contributing to an overall file size of approximately $120 \mathrm{MB}$. Data files are written in hierarchical data format, which allows for smaller file sizes as opposed to ascii files. All data files collected were backed up on FIU and PPPL servers. 


\section{CHAPTER 4}

\section{EXPERIMENT AND DATA COLLECTION}

This chapter concerns the experiments during which the diagnostic collects data, towards the end of the MAST 2013 operation.

\subsection{Plasma Pulse Characteristics}

The Mega Amp Spherical Tokamak (MAST), with a major radius of $0.85 \mathrm{~m}$ and minor radius of $0.65 \mathrm{~m}$, creates deuterium ohmic-heated plasmas. The average length of a plasma discharge is 0.5 seconds. During each plasma discharge, NBI heating can add from 1 megawatts (MW) to $4 \mathrm{MW}$ of power into the plasma. The neutral deuterium atoms in the neutral beam have an energy of around $70 \mathrm{keV}$. The plasma density is on the order of $10^{20}$ particles per cubic meter and the electron temperature of the plasma is usually a few $\mathrm{keV}$. The toroidal magnetic field of the plasma ${ }^{1}$ ranges from $0.5 \mathrm{~T}$ to $0.6 \mathrm{~T}$ and the plasma current ranges from $600 \mathrm{kA}$ to $900 \mathrm{kA}$. The total neutron rate, with NBI heating, is typically on the order of $10^{13} \mathrm{~Hz}$ or $10^{14} \mathrm{~Hz}$. Note that the FIDA and NC diagnostics collected data at the same time as the PD, which provides an opportunity for a combined analysis with multiple instruments.

An attempt to replicate various strong MHD activity scenarios, such as sawteeth and fishbones, was performed for several plasma discharges. One set of pulses at a fixed toroidal magnetic field was replicated so that the instruments could collect data from different regions of the plasma volume through scans of the position, sampled by changing the PD

\footnotetext{
${ }^{1}$ The experimental values for the toroidal magnetic field are values for $B_{\phi}$ at the magnetic axis. The magnetic axis is at the center of the plasma, where the value of the magnetic field decreases by a factor of $r^{-2}$ from the magnetic axis.
} 
position and $\mathrm{NC}$ orientation. The next set of pulses changed the toroidal magnetic field values while scanning the same region of the plasma volume. The plasma current was varied between $750 \mathrm{kA}$ and $900 \mathrm{kA}$ for another set of plasma pulses.[63][64]

A set of scenarios in which the plasma parameters were relatively constant and had low MHD activity were also replicated, these were called quiescent scenarios. A target current of $600 \mathrm{kA}$ and neutral beam energy of $65 \mathrm{keV}$ were used to create a quiescent plasma for a few hundred ms.[63][64] Additionally, the slowing down of beam ions after the NBI heating ended was studied. During these particular experiments two neutral beams were used in succession, called beam blips, so that two time intervals in which the NBI ends could be observed.

\subsection{Data Collection}

During data collection, the radial position of the RP (and therefore the radial position of the PD mechanical housing) was remotely controlled. When a trigger signal was received from the MAST Central Controller before the plasma pulse, the digitizer started to record analog voltage signals for the duration of the pulse. The digitizer sampling rate was 60 MHz. One data file, containing data for each of the four PD channels, was saved per plasma discharge. There was usually fifteen minutes between plasma discharges which allowed for the comparison of time stamps in data file names to the MAST list of experiment numbers (shots). Each plasma discharge was assigned a shot number, a unique six digit number to which the plasma discharge can be referred. A log of data files and corresponding shot numbers was kept and updated throughout data collection.

Data Acquisition Software A total of 43 GB of data was collected throughout the experiments, this included testing the equipment. The data acquisition software described in 
Chapter 3 was run remotely because safety protocols prohibit access to the MAST vessel during operation. The program was started in the morning, before experiments began, and was left on all day to record data whenever it received a trigger.

As previously described in Chapter 3, a $5 \mathrm{~V}$ digital signal (lasting $0.5 \mathrm{~s}$ ) was used as a trigger input signal. When the rising edge of the trigger signal was received from the MAST Central Controller before the plasma discharge (or pulse), the digitizer started to record voltage signals for the duration of the requested data collection time. During data collection the MAST trigger signal was sent $10 \mathrm{~ms}$ before the plasma discharge. The program controlled the digitizer such that it triggered on the rising edge of the signal and then waited $70 \mathrm{~ms}$ after receiving the trigger to start collecting data.

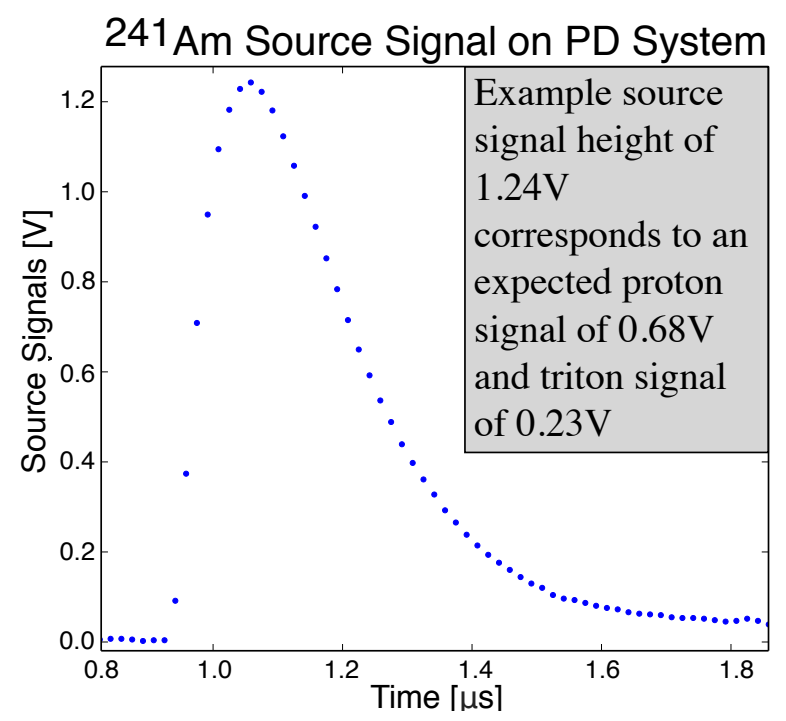

Figure 4.1: Source signals due to a $5.49 \mathrm{MeV}$ alpha-particle from the decay of a 241 Am source was used to test the PD system settings to calculate expected signal sizes for protons and tritons. The caption and figure are from Perez et al., Rev. Sci. Instrum. 85, 11D701 (2014).[55]

Radiation Source Expected signal sizes for protons are between $0.55 \mathrm{~V}$ and $0.71 \mathrm{~V}$ and for tritons are between $0.18 \mathrm{~V}$ and $0.24 \mathrm{~V}$, see Figure 4.1. This was determined using an Am-241 5.49 MeV alpha source to test the PD amplification and shaping electronics. 
Expected signal widths (FWHM) were around $300 \mathrm{~ns}$ using shaping amplifier integration and differentiation time constants of $100 \mathrm{~ns}$. Note that because of noise levels ranging from $0.05 \mathrm{~V}$ to $0.15 \mathrm{~V}$, helium-3 ions were not resolved as their expected pulse height of $0.15 \mathrm{~V}$ was on the order of the background noise levels. Tests using an Americium source were conducted before and after shipping electronics to the CCFE testing facility.

Raw Analog Data Saved waveform data were graphed with the NBI power. The DD fusion rate dependence on BT reactions were immediately evident, see Figure 4.2. When taking an initial look at the raw data (Figure 4.3) proton and triton pulses were observed. The analysis of these pulses will be discussed in Chapter 5 .

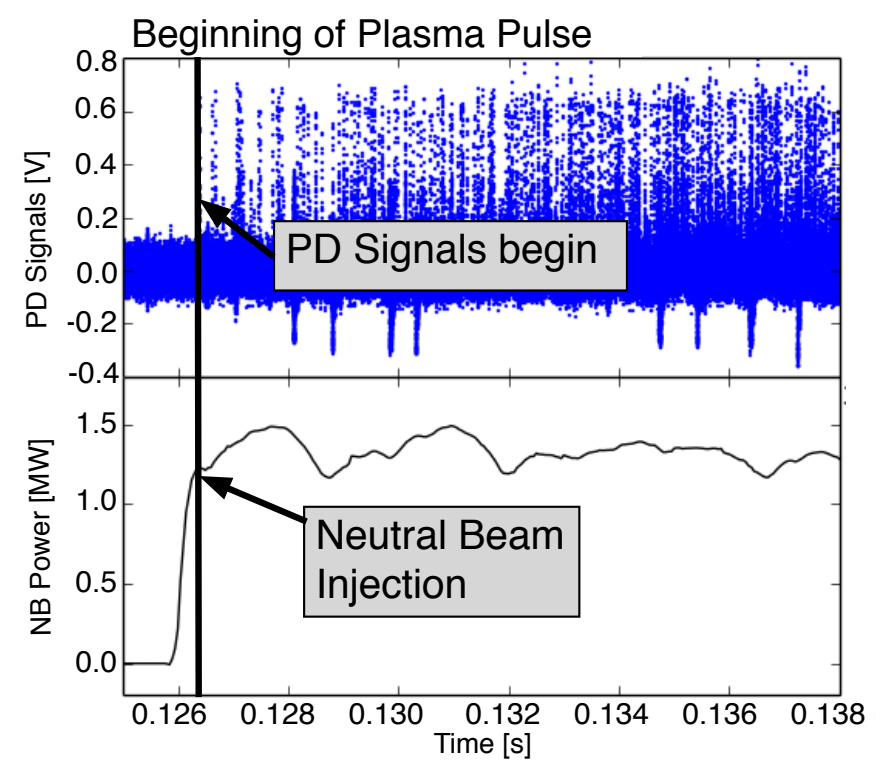

Figure 4.2: These are raw data from plasma pulse 29879, Channel 2. Fusion proton signals appeared as soon as the NBI heating was applied due to the beam-dominated nature of fusion reactions on MAST. The caption and figure are from Perez et al., Rev. Sci. Instrum. 85, 11D701 (2014).[55] 


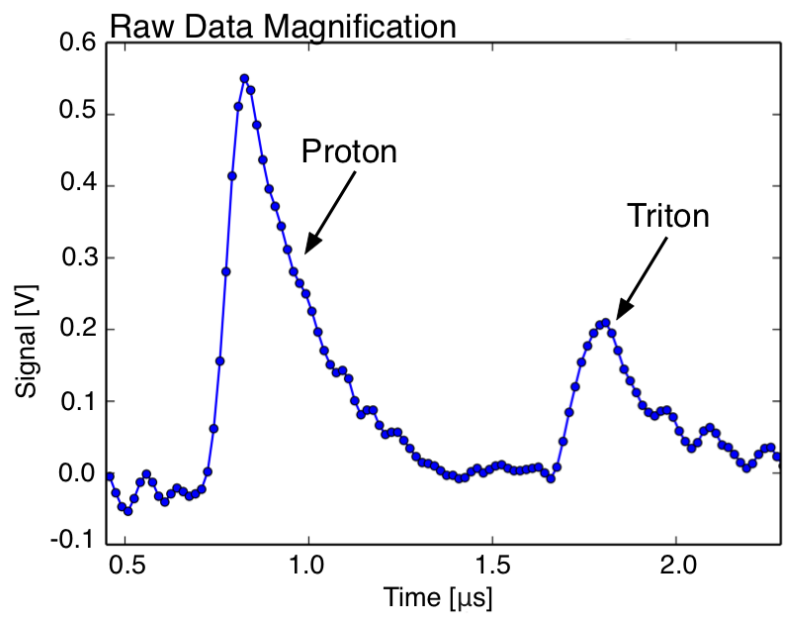

Figure 4.3: These are raw data from plasma pulse 29879, Channel 1. A magnification of data signals shows the characteristic shape of a fusion proton and triton. This finite time interval is not with respect to the beginning of the pulse. The caption and figure are from Perez et al., Rev. Sci. Instrum. 85, 11 D701 (2014).[55]

\subsection{RP Positions}

The MAST reciprocating probe (RP) arm is a pneumatic linear actuator with rotational capabilities ${ }^{1}$ about the mechanical arm's longitudinal axis. The RP system and supporting structure are displayed in Chapter 3. The PD's custom connector, built to MAST specifications, was attached to the end of the RP. The custom connector and locating pin ensured a fixed position giving the proper orientation for all PD channels. The distance from the center of the tokamak to the RP was calculated using equations and references provided by MAST colleagues. PD detector positions and orbit simulations discussed in Chapter 2 depend on this distance.

Remote Positioning The RP is installed such that its height lies along the mid-plane of the tokamak (meaning $\mathrm{z}=0$ or equatorial plane of the tokamak). Therefore, when remotely

\footnotetext{
${ }^{1}$ The rotational capabilities of the RP were not used during data collection.
} 
moving the RP towards or away from the center of the tokamak, only the radial position changes. The setpoint value, a distance in $\mathrm{mm}$, controls the radial position of the RP with respect to the center of the tokamak.

Because entrance is not allowed into the area surrounding the MAST vessel during operation, the RP position was remotely controlled. The setpoint value was input into the RP position control box. The maximum setpoint value (related to the farthest distance from the RP to the center of the tokamak) allowed was 500. Beyond this value remote control capabilities for the RP can be lost. Desired RP radial distances were calculated before each experiment.

Though the option of manually rotating the RP position about its radial axis was available (before and after MAST operations), it was not utilized. Note that 2001 is the last time the system for remotely controlling the RP position was calibrated.

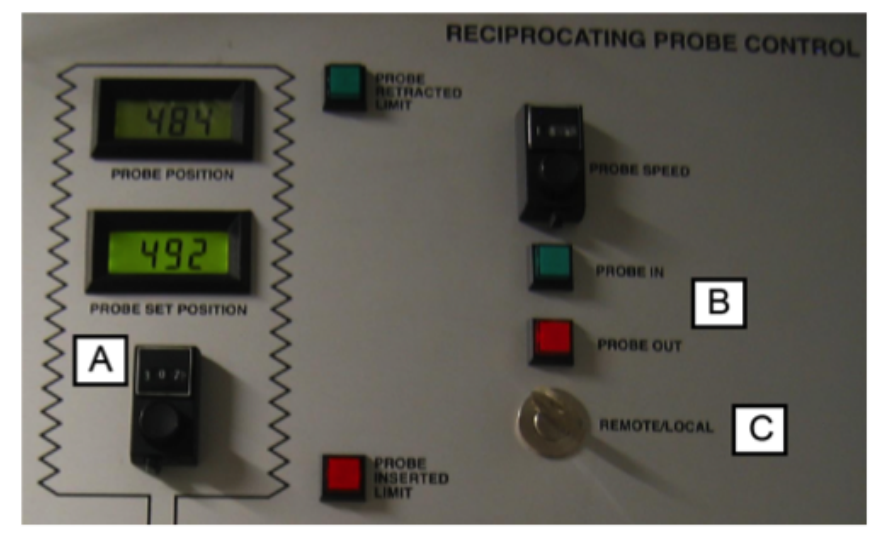

Figure 4.4: Above is an image of the RP position control box near the MAST control room. $A$ is the dial to set the RP position, $B$ is the button to press to move the RP towards or away from the center of the tokamak, and $C$ is the key to turn to make any changes to the RP position. Image courtesy of the CCFE.

Detector Positions Relative to PD Coordinate System The PD has four individually collimated detectors. The center of each collimator aligns with the center of its respective detector. Each detector is associated with one data channel and each has a unique angular 
orientation with respect to the PD coordinate system. Table 4.1 summarizes the individual detector orientations within this PD coordinate system as described in Chapter 2.

\begin{tabular}{|c|c|c|c|}
\hline Channel & Detector & Phi $(\mathrm{deg})$ & Theta $(\mathrm{deg})$ \\
\hline 0 & 4 & -45 & 40 \\
\hline 1 & 1 & -30 & 40 \\
\hline 2 & 2 & -35 & 40 \\
\hline 3 & 3 & -40 & 40 \\
\hline
\end{tabular}

Table 4.1: This table lists individual channel (and detector) angular orientations within the PD coordinate system.

Detector Positions Relative to Center of Tokamak The setpoint values, described previously, were used to calculate the distance of the PD Channel 0 collimator entrance with respect to the center of the tokamak. Therefore, using the setpoint values and the distances of all the channel collimator entrances with respect to each other, it is possible to calculate the distance of each channel's collimator entrance with respect to the center of the tokamak (Table 4.2). These collimator entrance distances were used as inputs to the particle trajectory simulations. Figure 4.5, Figure 4.6, and Figure 4.7 display each channel's collimator entrance with respect to the $\mathrm{X}(\mathrm{R}), \mathrm{Y}$, and $\mathrm{Z}$ axes.

\begin{tabular}{|c|c|c|c|c|}
\hline Channel & Detector & $\begin{array}{c}\text { Radial } \\
\text { Distance }(\mathrm{x}, \mathrm{R})\end{array}$ & $\begin{array}{c}\text { Vertical } \\
\text { Distance }(\mathrm{z})\end{array}$ & $\begin{array}{c}\text { Horizontal } \\
\text { Distance }(\mathrm{y})\end{array}$ \\
\hline 0 & 4 & Calculated RP distance & $27.5 \mathrm{~mm}$ & $-25.8 \mathrm{~mm}$ \\
\hline 1 & 1 & Ch0 Distance $-270.8 \mathrm{~mm}$ & $31.1 \mathrm{~mm}$ & $-30.6 \mathrm{~mm}$ \\
\hline 2 & 2 & Ch0 Distance $-277.4 \mathrm{~mm}$ & $14.6 \mathrm{~mm}$ & $-39.1 \mathrm{~mm}$ \\
\hline 3 & 3 & Ch0 Distance $-290.5 \mathrm{~mm}$ & $41.6 \mathrm{~mm}$ & $-14.3 \mathrm{~mm}$ \\
\hline
\end{tabular}

Table 4.2: This table lists individual channel (and detector) collimator entrance distances relative to the center of the tokamak. 


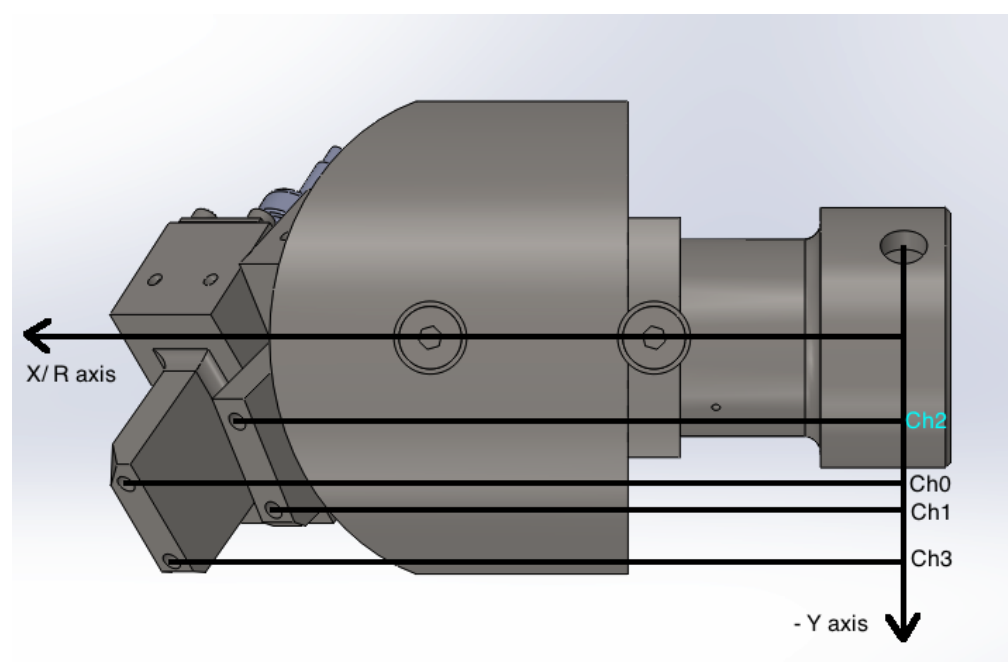

Figure 4.5: The image above displays the radial offsets of detector collimator entrances to the RP attachment point. The y-axis is vertical, r-axis is horizontal, and - $\mathrm{z}$-axis goes into the page.

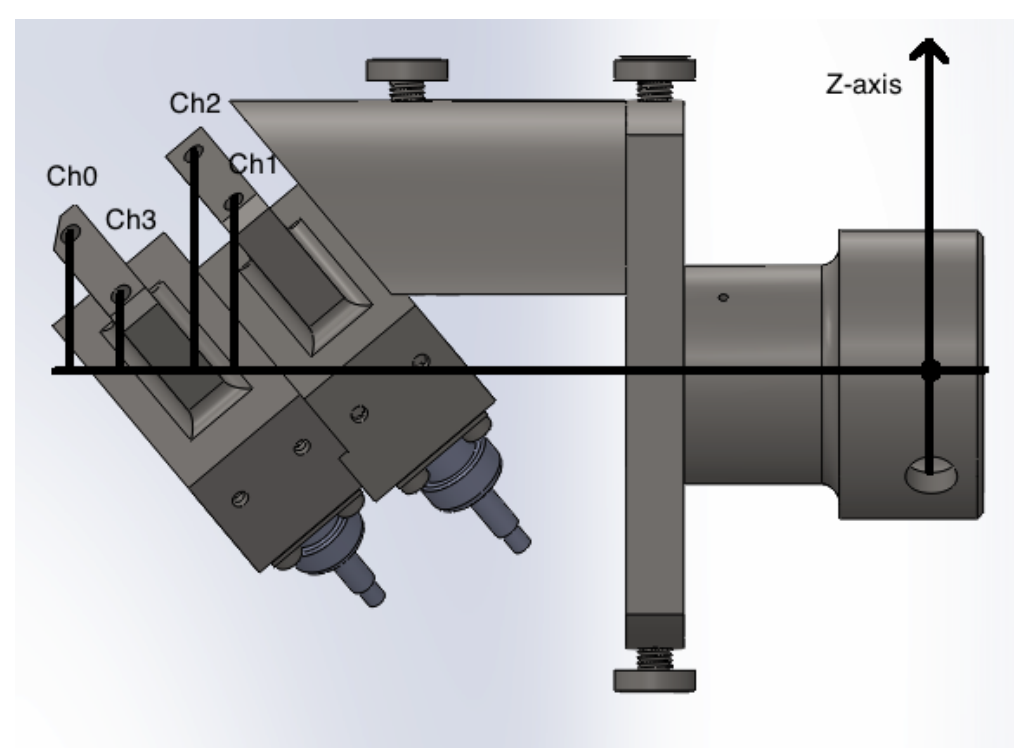

Figure 4.6: The image above displays the vertical offsets of detector collimator entrances to the RP attachment point. The z-axis is vertical, r-axis is horizontal, and $+y$-axis goes into the page.

\subsection{Detector Response to Varying Particle Rates}

Certain time intervals during the waveform data indicated a possible overload of the detector (mostly Channel 0) or the detector electronics (Figure 4.8). It was hypothesized 


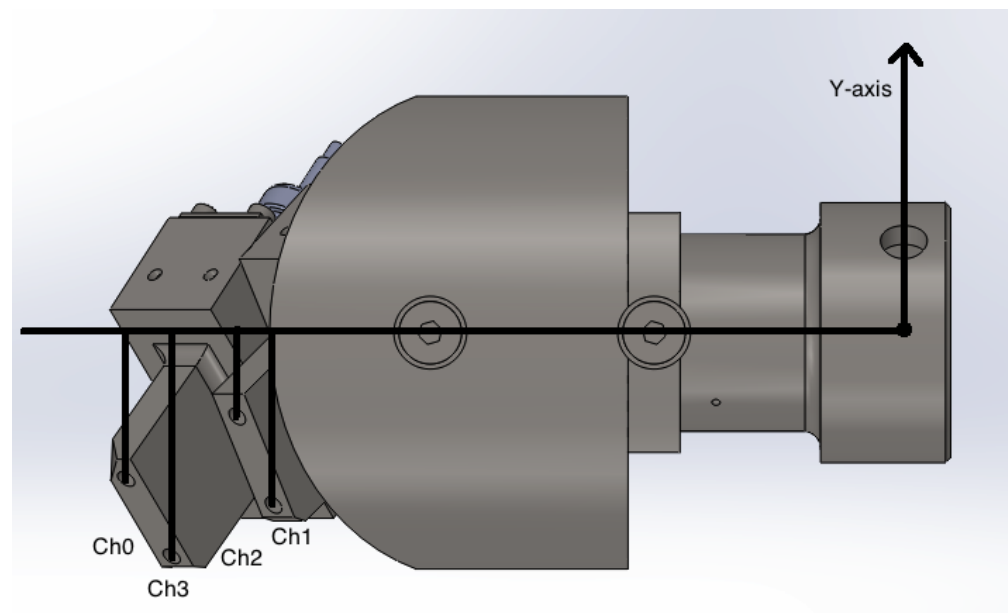

Figure 4.7: The image above displays the horizontal offsets of detector collimator entrances to the RP attachment point. The y-axis is vertical, r-axis is horizontal, and - z-axis goes into the page. Note that the $+y$-axis is up and $\mathrm{y}$-axis is down.

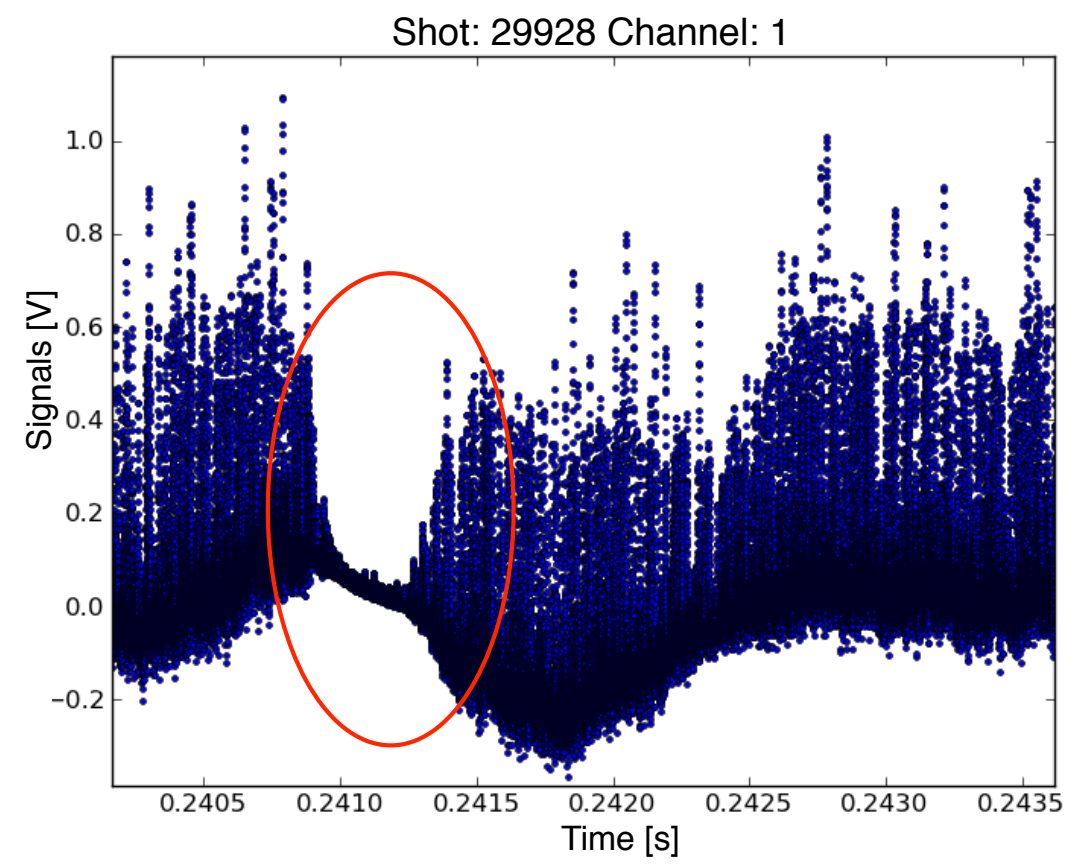

Figure 4.8: There were periods during the data collection, circled in red in the analog data above, that indicated a possible overload or saturation of electronics.

that these time intervals were caused by high particle rates. Therefore an investigation of the optimal particle rates for the detector and associated electronics was conducted. An 
experiment at the Florida State University Linear Particle Accelerator facility (FSULP) in Tallahassee, Florida was conducted. The facility's Van de Graaf generator was used to create a $4 \mathrm{MeV}$ proton beam scattering experiment (see Figure 4.9) to test the PD electronics used during the MAST experiments. In this experiment, the angle between the variable detector and the beam was varied to change the observed particle rates. A control detector, or monitor detector, remained fixed at one angle in reference to the beam. Over one dozen scattering experiments lasting less than $0.5 \mathrm{~s}$ each were performed at varying detector angles, using digitizer sampling rates of $30 \mathrm{MHz}$ and $60 \mathrm{MHz}$. Before conducting the experiments, a Thorium-228 5.5 MeV alpha source was used to check the electronics.

The same detectors, aluminum foil filters, and amplification electronics used at MAST were used at FSULP. The collimator size for the experiment and gold target thickness were chosen such that when the variable detector was changed from $30^{\circ}$ to $6^{\circ}$ relative to the incident beam, particle rates from several $\mathrm{kHz}$ to over $500 \mathrm{kHz}$ were expected. The variable detector was placed $17 \mathrm{~cm}$ from the target and the exposed active area ${ }^{1}$ of the detector was $4.77 \mathrm{~mm}^{2}$. A beam current of $8 \mathrm{nA}$ and a gold foil target, $330 \mu \mathrm{g} / \mathrm{cm}^{2}$ thick, was used as well. The expected particle rates recorded by the detectors can be expressed by,

$$
\frac{d N_{D}}{d t}=\frac{d N_{B}}{d t} N_{T} \sigma_{B T} \delta \Omega_{D}
$$

where Rutherford scattering (proportional to $\sin ^{-4} \theta$ ) was used to calculate the cross section between the beam and the target $\sigma_{B T} .^{2}$

Software was written in LabVIEW to collect variable and monitor detector voltage signals as well as display a rough approximation of detected particle rates in real time during data collection. Figure 4.10 displays the energy spectra, or histogram of particle pulse

\footnotetext{
${ }^{1}$ The square collimator geometry had dimensions of $4.77 \mathrm{~mm}$ by $1 \mathrm{~mm}$.

${ }^{2}$ The number of target nuclei per unit area is represented by $N_{T}$, the incident beam particle rate is represented by $d N_{B} / d t$, and the solid angle of the detector is represented by $\delta \Omega_{D}$.
} 


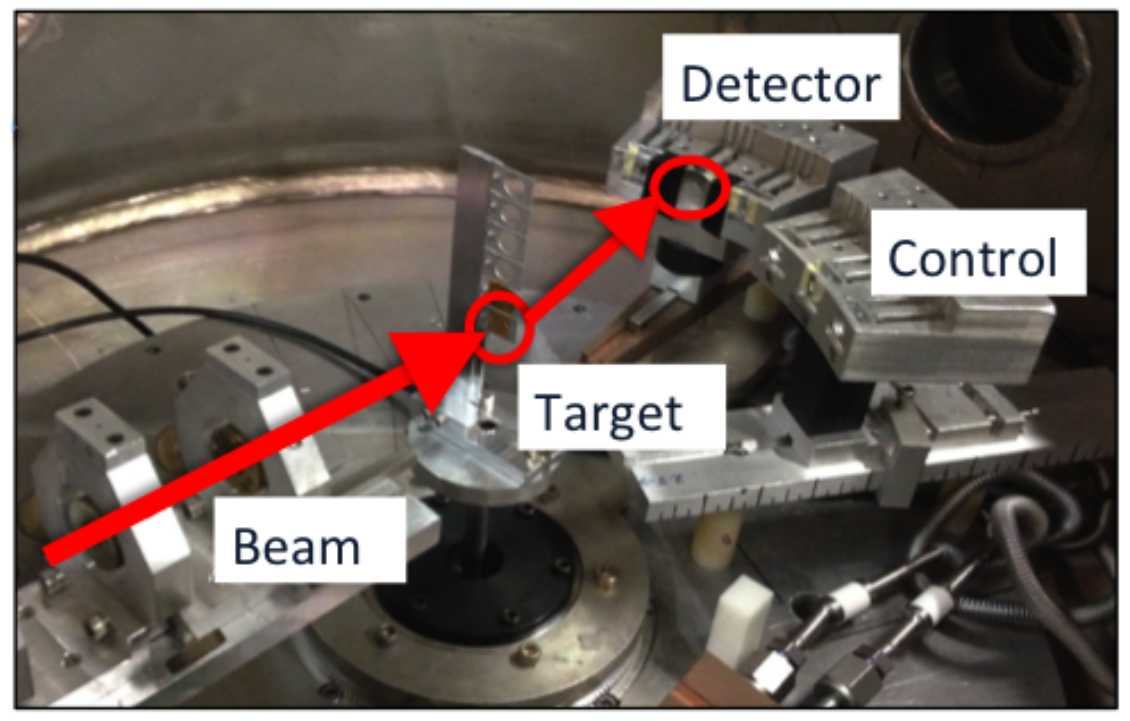

Figure 4.9: A scattering experiment with a $4 \mathrm{MeV}$ proton beam and gold target were used to test the PD electronics during varying particle rates. In this experiment, the angle between the variable detector and the beam was varied to change the observed particle rates. A control detector, or monitor, remained fixed at one angle in reference to the beam.

heights, for the variable detector (in red) and the monitor detector (in blue) boxed in red during one experiment. ${ }^{1}$ In the figure, the monitor detector's energy spectrum (blue) covers a smaller voltage range than the variable detector's (red) energy spectrum. The different energy spectra occur because the variable detector is exposed to a higher incident particle rate (above $300 \mathrm{kHz}$ ) than the monitor detector $(2 \mathrm{kHz})$. The difference in particle rates between the two detectors is also reflected in the waveform data of voltage signals, which can be seen in the graph above the energy spectra in Figure 4.10.

\footnotetext{
${ }^{1}$ The figure is a screenshot that was taken during data collection.
} 


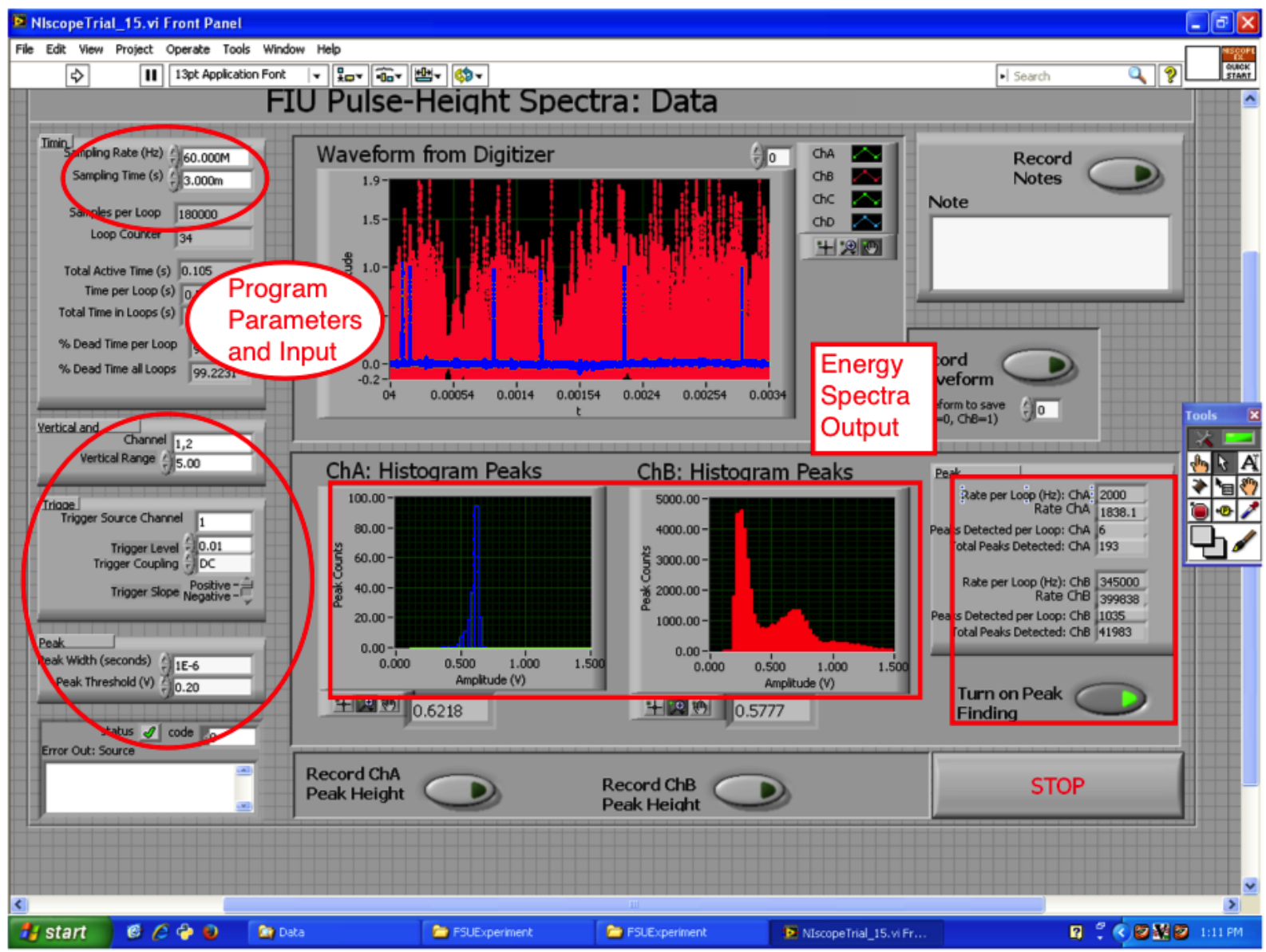

Figure 4.10: Data acquisition software was written in LabVIEW to collect detector voltage signals as well as display an approximate rate of particles detected $(\mathrm{Hz})$ in real time during data collection. The approximate particle rates for both the variable detector $(\mathrm{ChB})$ and the monitor detector $(\mathrm{ChA})$ were displayed.

As particle rates increased to above $200 \mathrm{kHz}$, a significant amount of pileup occurred, as can be seen in the waveform data of Figure 4.10. Pileup ${ }^{1}$ can occur if two particles hit the detector within a close enough time interval and are subsequently recorded as a single pulse (with a larger amplitude than a single pulse). Large amplitude signals caused by pileup are reflected in a wider energy spectrum for the variable detector. A high rate of incident particles also caused more background noise which further contributed to the range

${ }^{1} \mathrm{~A}$ specific example of pileup is discussed in Chapter 5 . 
of the variable detector's energy spectrum. Figure 4.11 shows images of an oscilloscope (displaying the analog detector signal) at various particle detection rates displayed at: 6 $\mathrm{kHz}, 285 \mathrm{kHz}$, and $425 \mathrm{kHz}$. These images show how pulse signals can degrade (in both amplitude and width) as the electronics become saturated.

Additionally, high leakage currents can cause charge build up on the surface of the silicon surface barrier detectors. Charge build up on these types of detectors can further degrade signals through adding background noise and increasing the temperature of the detectors themselves. Leakage currents from 50 nA to over 200 nA were observed. Smaller scattering angles between the beam and variable detector resulted in higher leakage current values.

The energy spectra (of pulse amplitudes) for each detector were integrated to calculate the experimental particle rates. To account for the uncertainty in the scattering angle the variable detector rates were divided by the monitor detector rates to create the normalized detector rates, $R_{e x p}$. Figure 4.12 displays fitting a $\sin ^{-4}(\theta / 2)$ function to the angular distribution (scattering angle with respect to the beam) of normalized rates for the variable detector. Even though particle rates over $400 \mathrm{kHz}$ were detected, the $R_{\exp }$ function followed the expected $\sin ^{-4}(\theta / 2)$ trend,

$$
R_{f i t}=\frac{C_{0}}{\sin ^{4}\left(\frac{\theta-\theta_{0}}{2}\right)}
$$

where $C_{0}$ and $\theta_{0}$ are fitting parameters. ${ }^{1}$ Fluctuations between $R_{f i t}$ and $R_{\text {exp }}$, as seen in the bottom graph of Figure 4.12, indicate a lack of control in the beam direction.

Operating the electronics above several hundred $\mathrm{kHz}$ resulted in regions of saturated waveform signals as observed in MAST data, making high particle rates and leakage

\footnotetext{
${ }^{1}$ The value of $C_{0}$ is $0.006+/-0.001$ and $\theta_{0}$ is $0.028+/-0.006$.
} 
currents a plausible explanation for signals as displayed in Figure 4.8. For future experiments, implementing smaller collimator diameters into the probe head design can reduce the particle rates incident on the detectors.

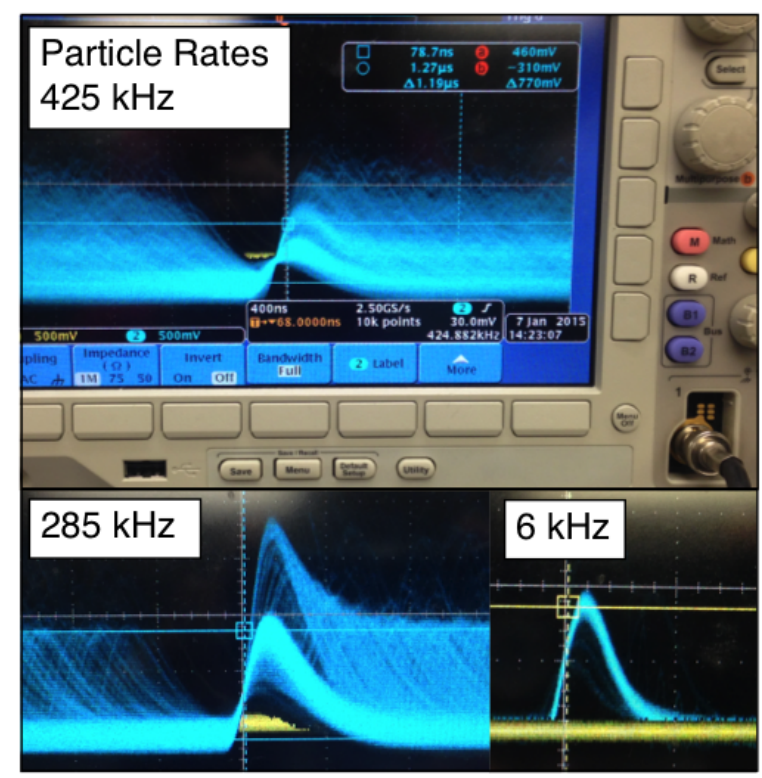

Figure 4.11: Images of an oscilloscope at various incident particle detection rates are displayed at: $6 \mathrm{kHz}, 285 \mathrm{kHz}$, and $425 \mathrm{kHz}$. The degradation of the pulse shape is greatest at $425 \mathrm{kHz}$. 

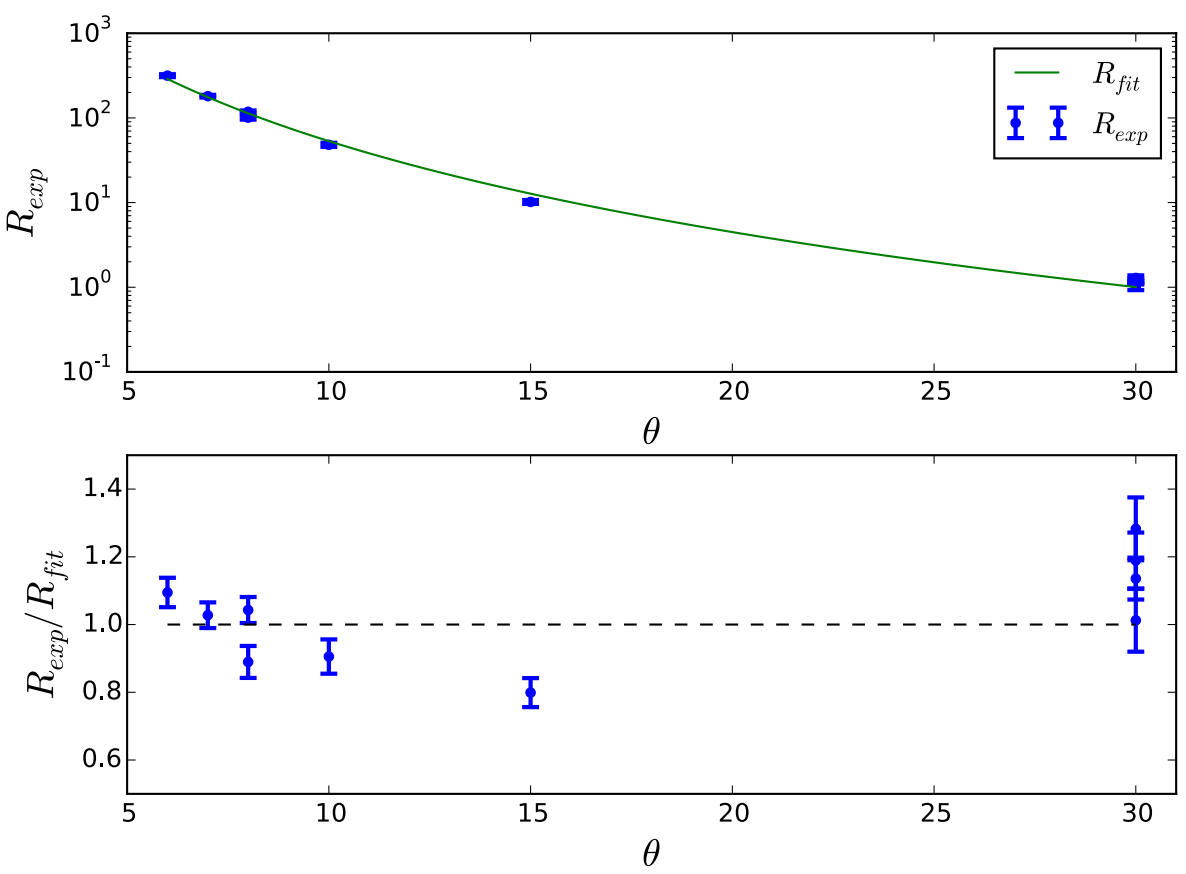

Figure 4.12: The normalized experimental particle rates are graphed above. Fluctuations between the fit and experimental data indicate a lack of control in the beam direction. 


\section{CHAPTER 5}

\section{DATA ANALYSIS}

The raw data consists of the amplifier output voltage corresponding to the amplified signal size of samples taken every 16.7 nanoseconds (ns). The digitizer sampling rate is 60 MHz. ${ }^{1}$ Processing the raw data to create proton production rates as a function of time will be discussed in this Chapter. The analysis involves two main procedures, indicated in Fig 5.1. The first procedure, highlighted in red, is to identify particle pulses above the electrical noise background and to determine their pulse amplitude in the data set. The second procedure, highlighted in green, is to integrate time dependent particle energy spectra, spectra which are created from the fitted data. The time dependent energy spectra are created by histogramming the extracted pulse heights for a sequence of time intervals. The result is a sequence of energy spectra. From the spectra, protons resulting from DD fusion reactions can be identified and counted.

\subsection{First Step: Fit Particle Pulses}

Pulses Figure 5.2 displays proton and triton signals observed in the raw data files. The average proton and triton height is approximately $0.6 \mathrm{~V}$ and $0.2 \mathrm{~V}$, respectively. As discussed in Chapter 3, these pulses are within the predicted signal height range using the PD data acquisition system. Their initial energies are described by (5.1). The full width of the pulse at half of its amplitude, FWHM, is approximately $300 \mathrm{~ns}$.

$$
D+D=P(3.02 M e V)+T(1.01 M e V)
$$

\footnotetext{
${ }^{1}$ This is also referred to as 60 Msamples per second.
} 


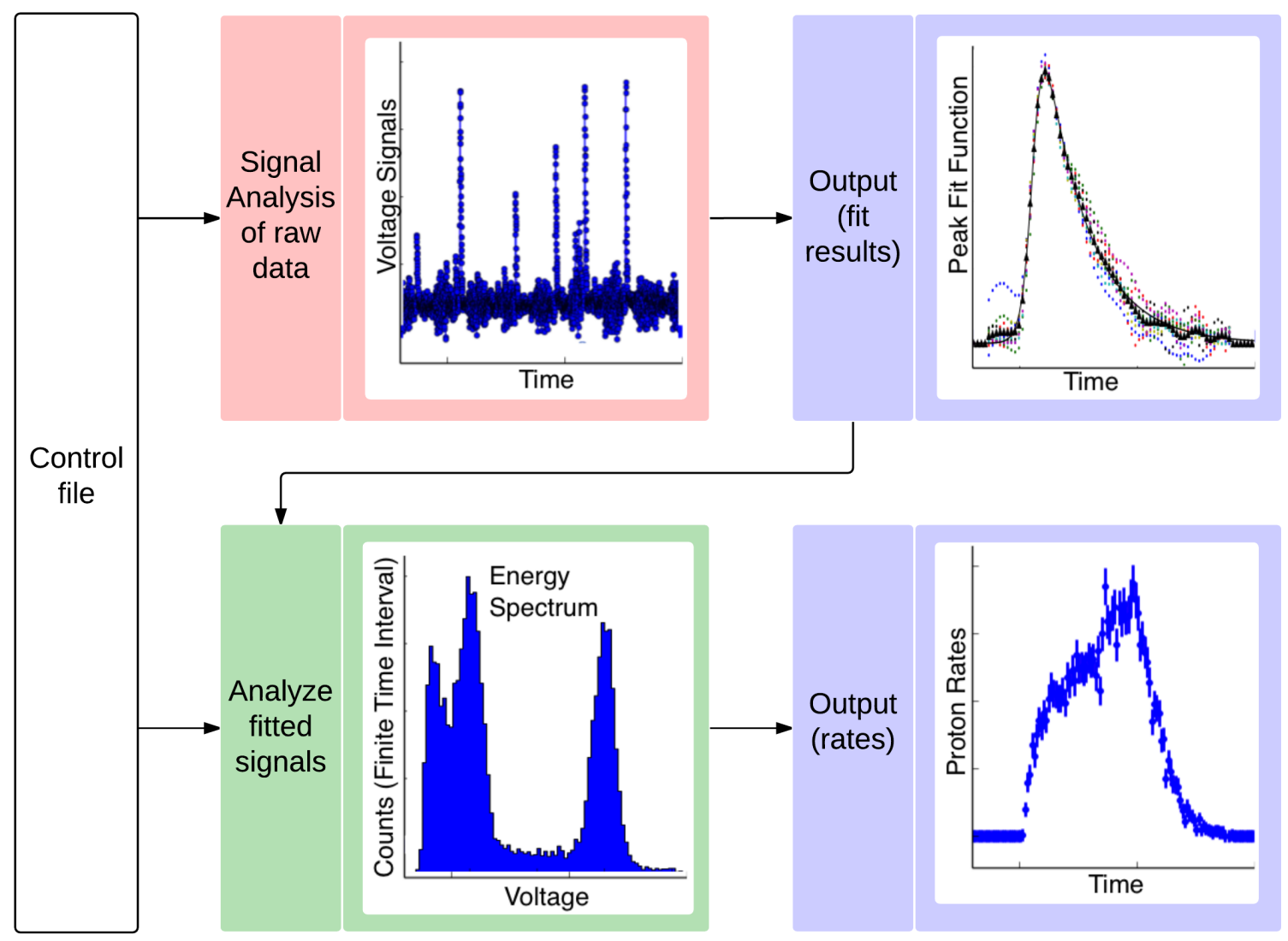

Figure 5.1: Above is a schema of the data analysis code and sample plots representing the results of each of the two stages of the analysis.

High particle rates, also discussed in Chapter 4, can cause fluctuating baselines as seen in Figure 5.3. Using simple pulse-height discrimination (PHD) methods with fluctuating baselines can cause a signal amplitude to be less than, or more than, its actual height depending on the vertical offset from the baseline. Without correcting for these baselines, an incorrect signal amplitude can interfere with signal identification using pulse height. Pileup can also occur during high particle rates. Pileup can happen when more than one particle hits the detector within a time interval lower than the pulse resolution time (the FWHM value of $300 \mathrm{~ns}$ ). For example, if a second pulse hits the detector before the detector has a chance to finish collecting charge from a previous pulse then the second pulse can appear at the tail 


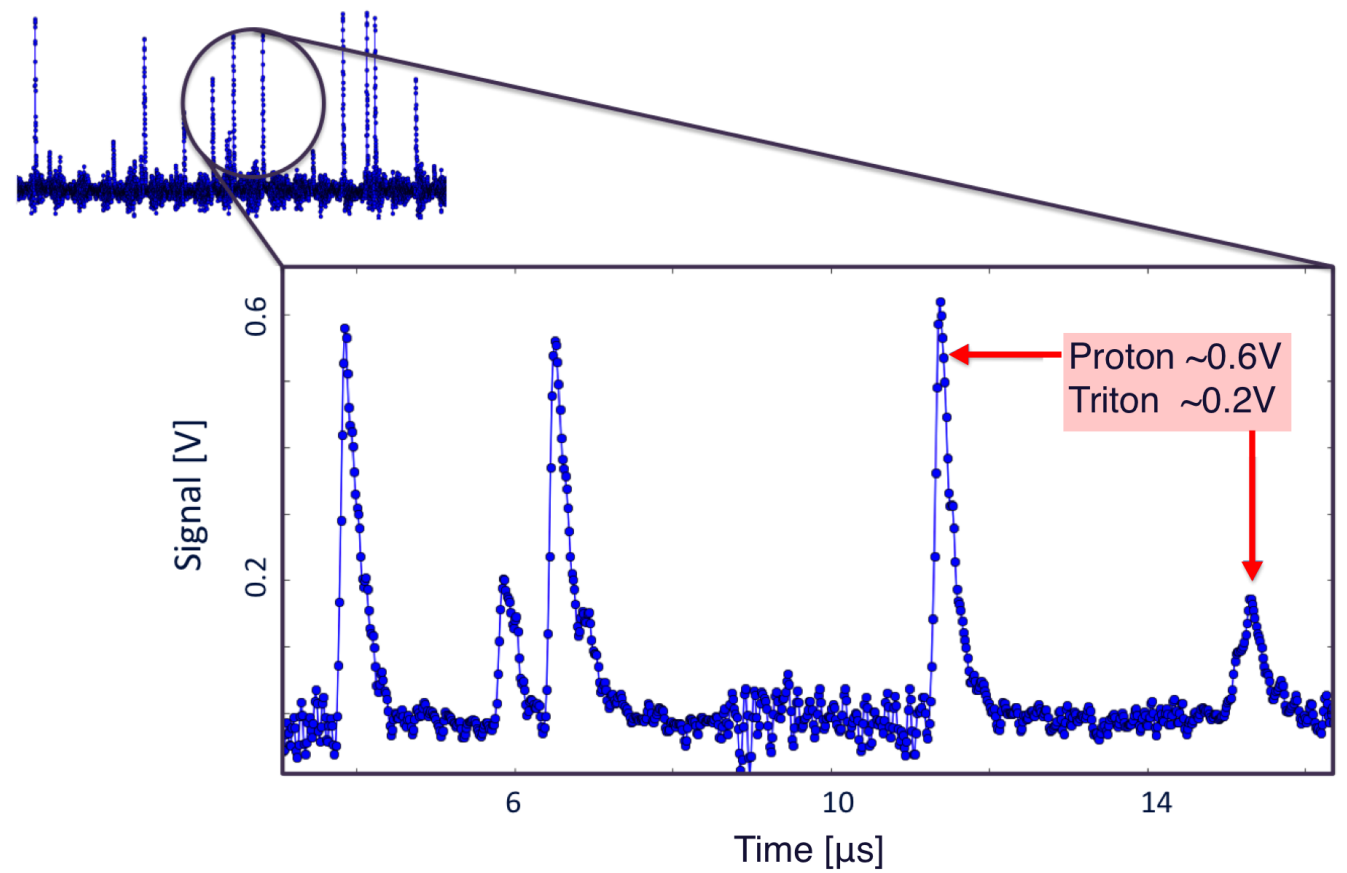

Figure 5.2: Characteristic proton and triton signals were observed in the raw data files.

end of the first pulse's signal. The pileup example is displayed in Figure 5.5. Additionally, if two particles hit the detector within a close enough time range, then the two pulses can be recorded as a single pulse which is characterized by an incorrect high amplitude. The second pileup example is displayed in Figure 5.6. Furthermore, high amplitude and high frequency noise (on the order of particle rates of several hundred $\mathrm{kHz}$ ) preclude the use of PHD methods since noise signals could be counted as real events. Figure 5.4 displays an example of this noise which necessitates fitting the data to distinguish between real events and background noise. 


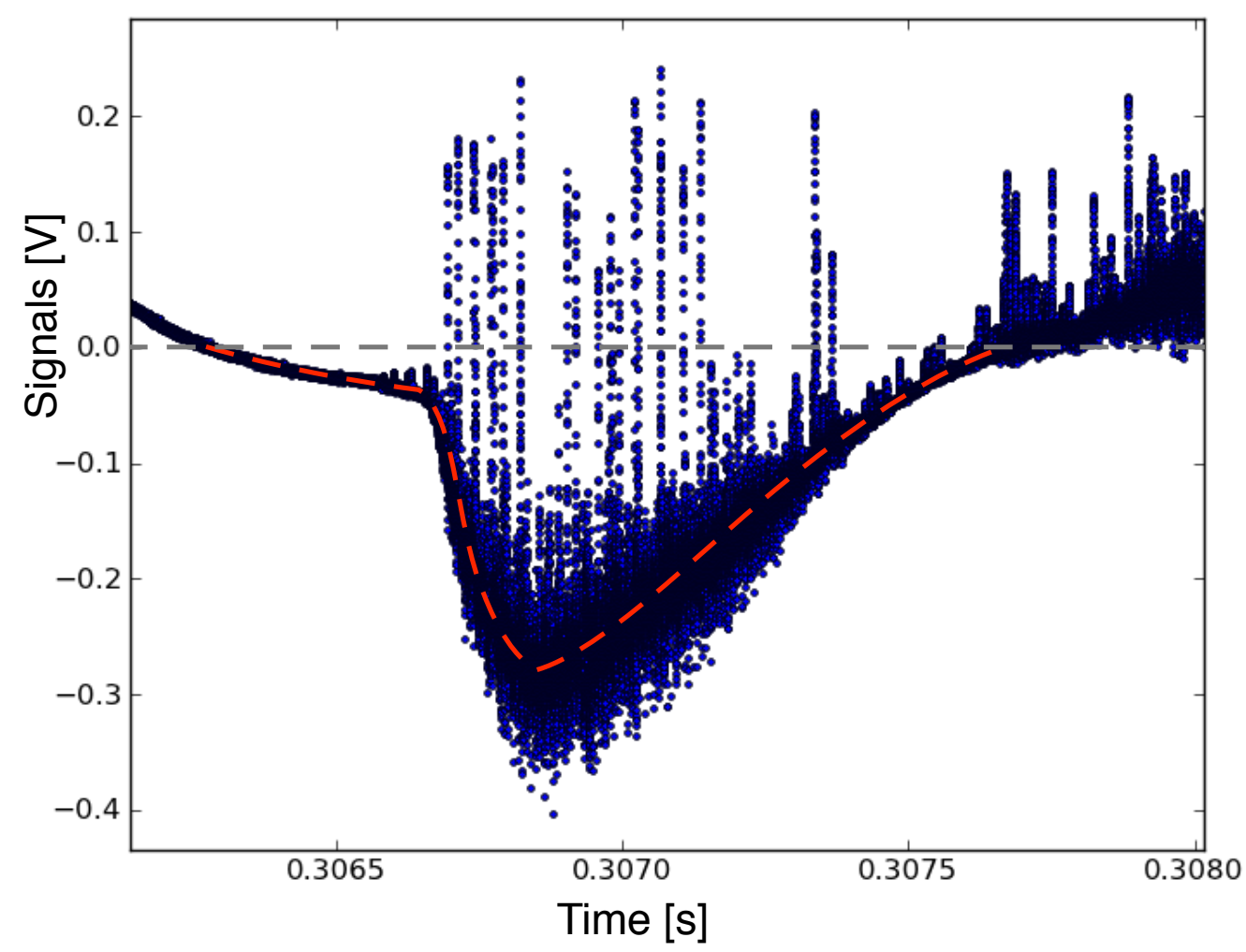

Figure 5.3: Fluctuating baselines necessitate fitting the data to better determine pulse amplitudes. The red dashed line outlines the fluctuating baseline between $0.3065 \mathrm{~s}$ and $0.3075 \mathrm{~s}$ compared to a non-fluctuating baseline drawn in a dashed grey line. Data used are from Shot 29906 Channel 1. 


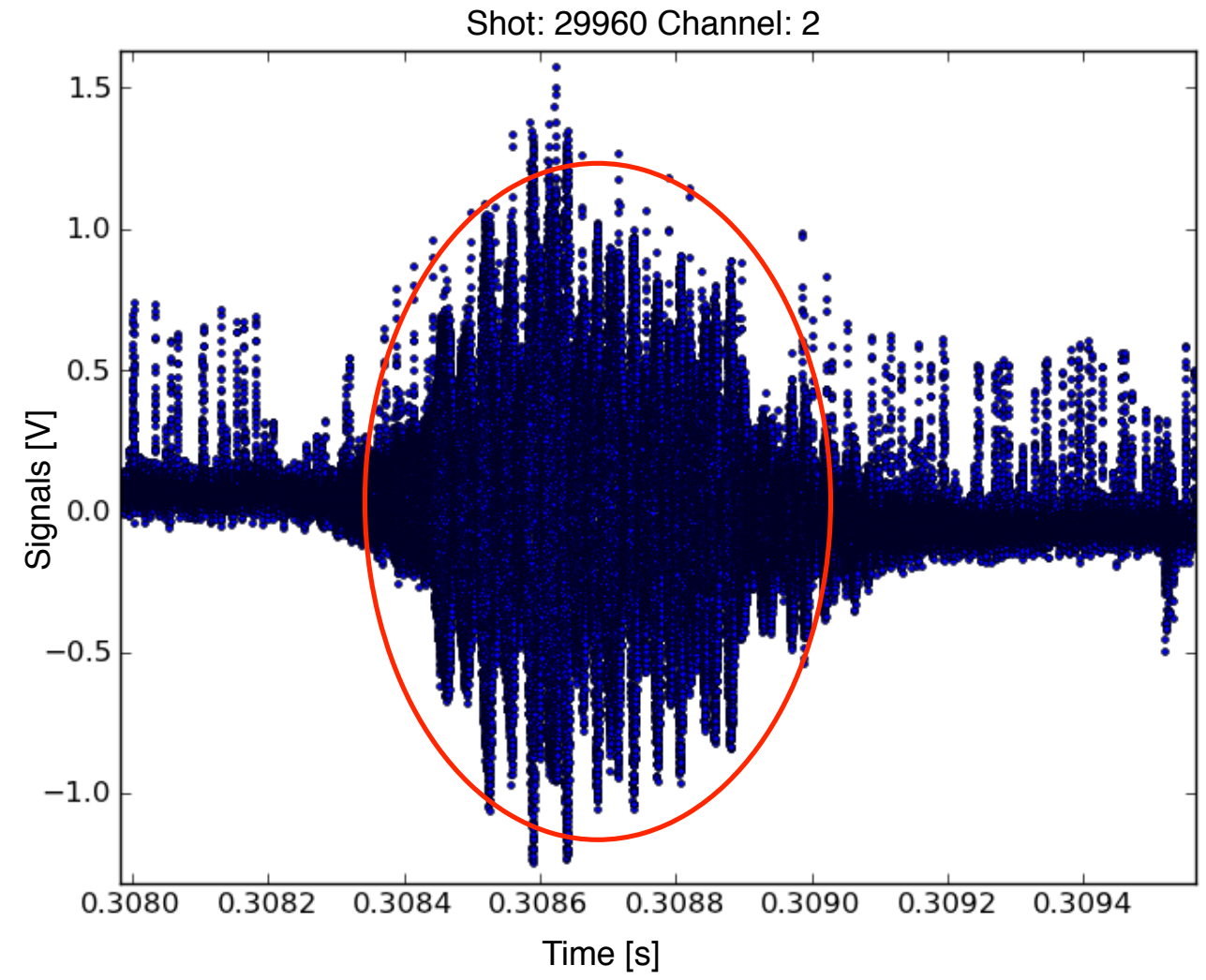

Figure 5.4: High frequency signals (circled in red) necessitate fitting the data to distinguish between real events and background noise. 


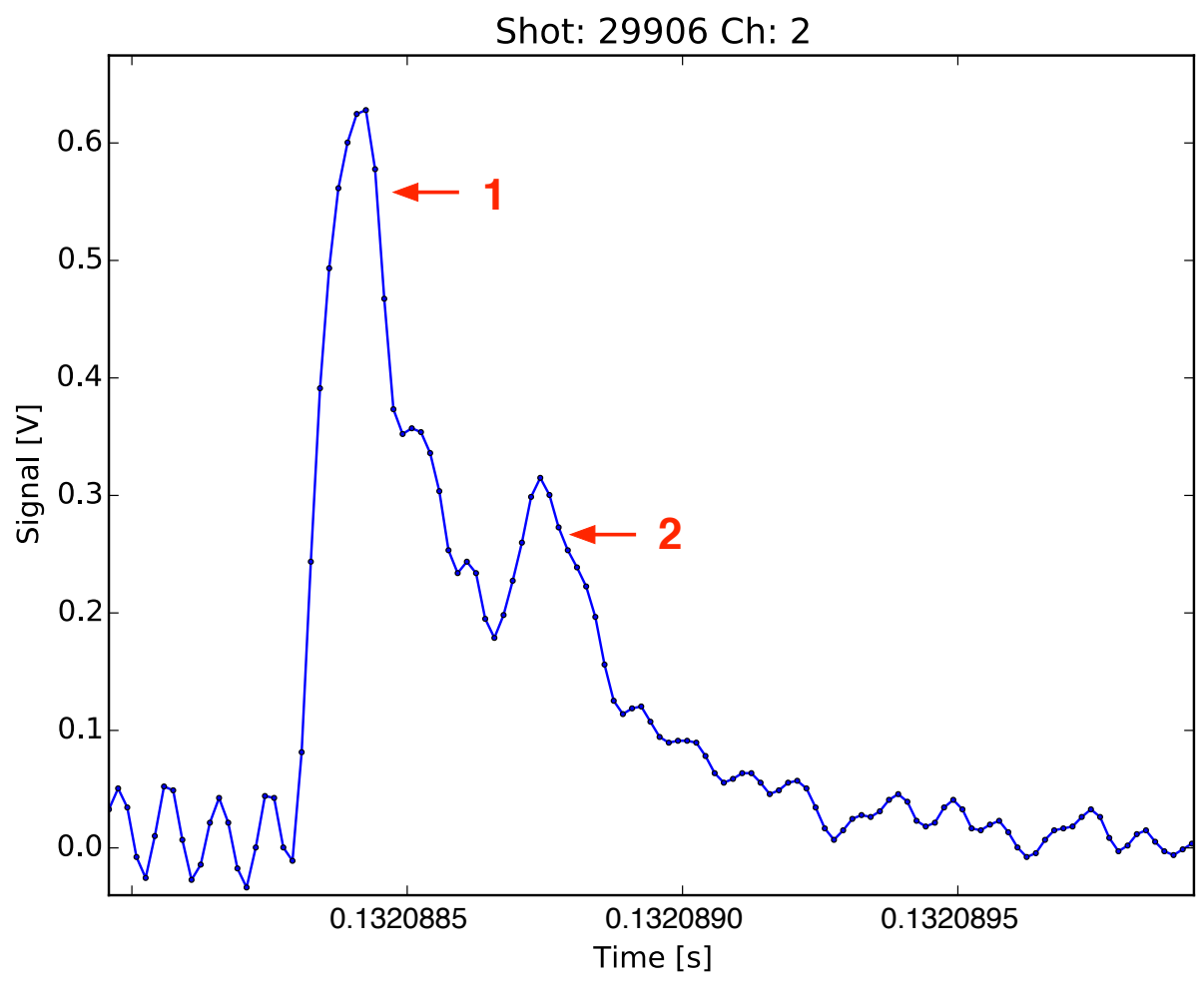

Figure 5.5: Above is an example of pileup occurring during high particle rates incident on the detector. The second pulse is observed on the tail of the signal of the first pulse. 
Shot: 29906 Ch: 2

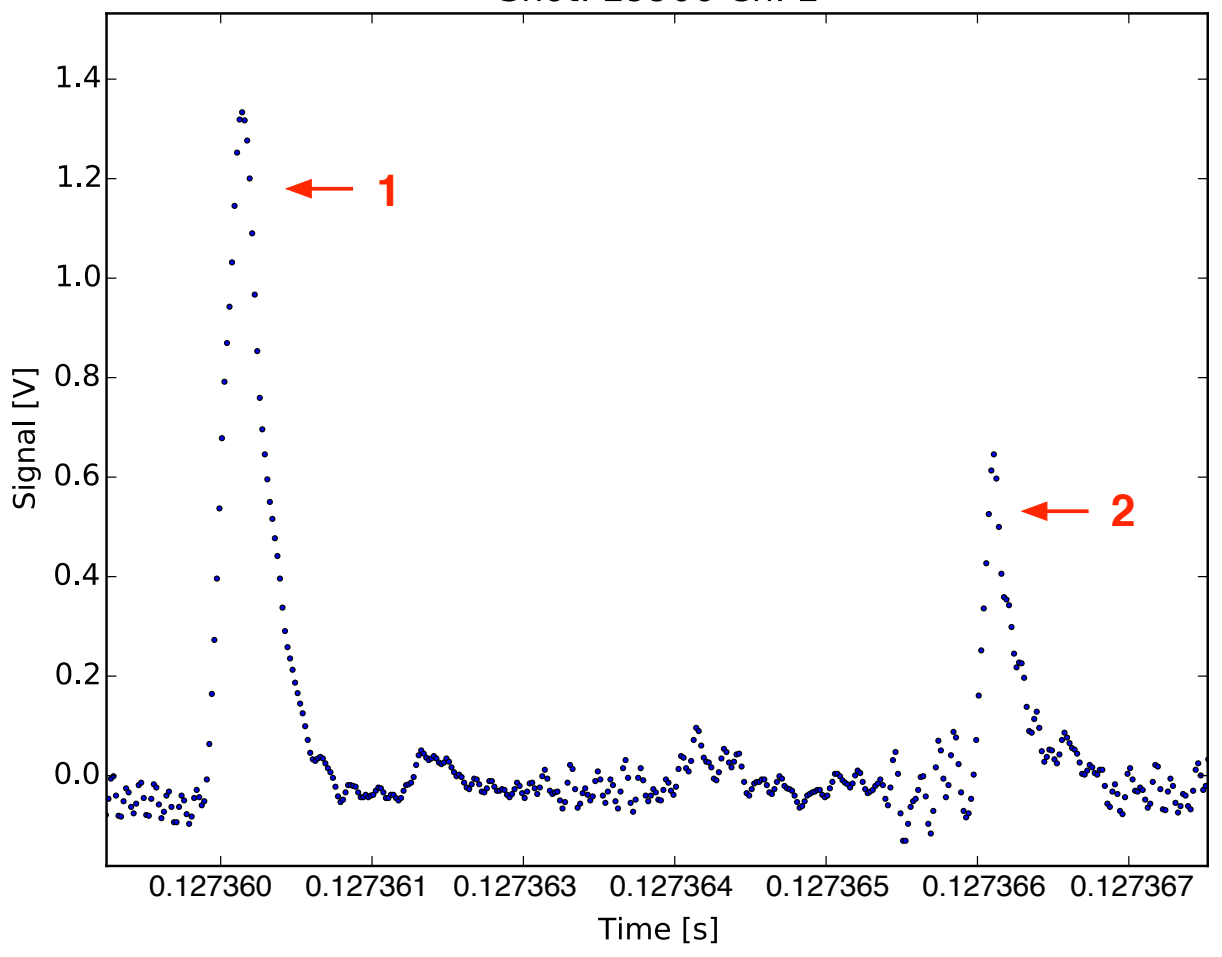

Figure 5.6: Above is another example of pileup. The first pulse results when two proton pulses hit the detector within a time interval less than the pulse time resolution of the detector. The second pulse is a proton pulse with the characteristic proton height. 
Selecting Peak Samples The first portion of the analysis uses a set of sample peaks which are fit to a general pulse-shape function, see Figure 5.7. The function represents a normalized standard peak shape,

$$
V=C_{0} e^{-C_{1}\left(t+t_{0}\right)}\left(t+\tanh \left(C_{2}\left(t+t_{0}\right)\right)\right)
$$

where $C_{0}$ is a calculated normalization factor (one divided by the peak height). The constants $C_{1}, C_{2}$, and $t_{0}$ are fitting parameters. The set of sample pulses are representative of the average pulse shape found throughout the data channel. The sample pulses are manually selected for each individual channel, see Figure 5.8. Figure 5.9 displays the individual data points and average shape of the sample peaks plotted along with the fit function.
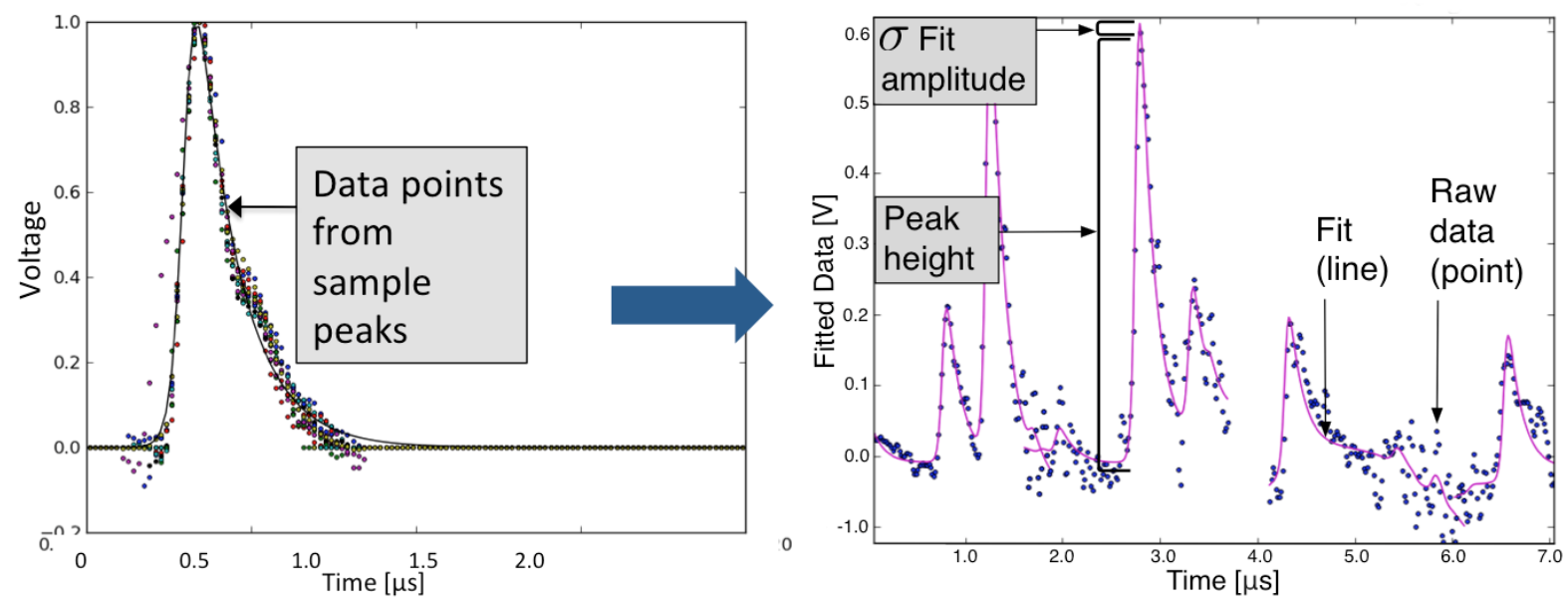

Figure 5.7: A set of sample peaks from a channel are chosen and fit to a general pulse-shape function. The analysis code then iterates through the raw data, fitting pulse-shape functions (with a quadratic background) to peaks. The Figure on the right is from Perez et al., Rev. Sci. Instrum. 85, 11D701 (2014). [55]

Fitting the Data Before the data are fitted, the raw data file is split into finite time intervals. This first analysis code iteratively fits data in these finite time slices, as shown in gray in Figure 5.10. After the initial fitting, new finite time intervals (slices shown in red in Figure 5.10) are chosen such that they span the edges of the first set of time intervals. Finally, 

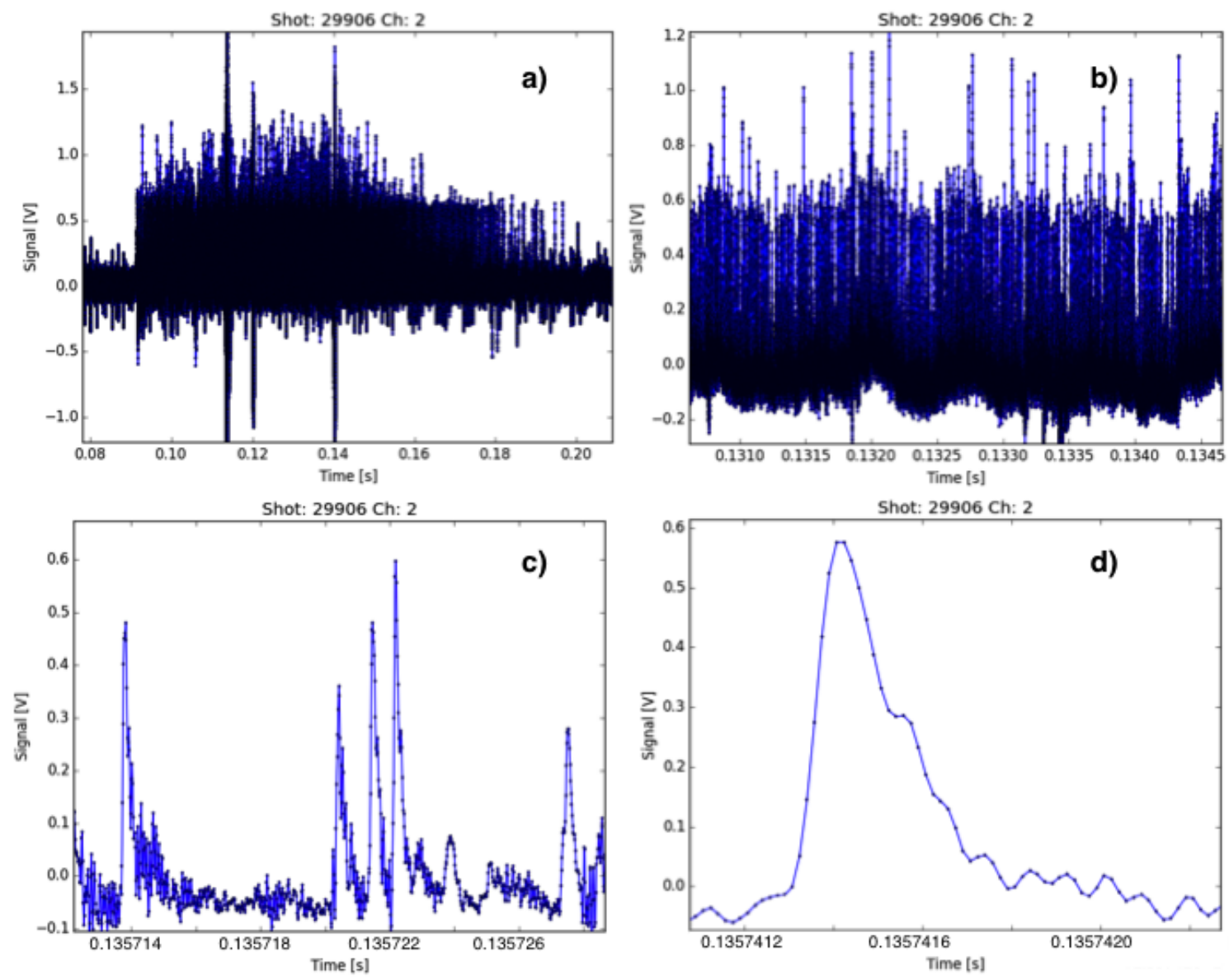

Figure 5.8: Time intervals for sample peaks, representative of the types of pulses found throughout the channel, are selected. Figure a) displays data for an entire plasma discharge (0.12 s) while Figure b) and Figure c) display closer views of the data $(3.5 \mathrm{~ms}$ and $15 \mu$ s time intervals). A single peak is displayed in d) which spans a $1 \mu$ s time interval.

the central region of each fitted time interval is adjoined to create one file of continuously fitted data. Using only the central region avoids retaining artifacts occurring from peak fits at the edge of time slices.

An algorithm then searches through all of the signals and fits peaks above a minimum threshold voltage value. Note that the peak positions are fixed during this procedure. Additionally, the peaks are fitted against a quadratic background to take into account fluctuating baselines. As previously mentioned, the procedure of fitting data is implemented to 
Shot: 29975 Channel: 1

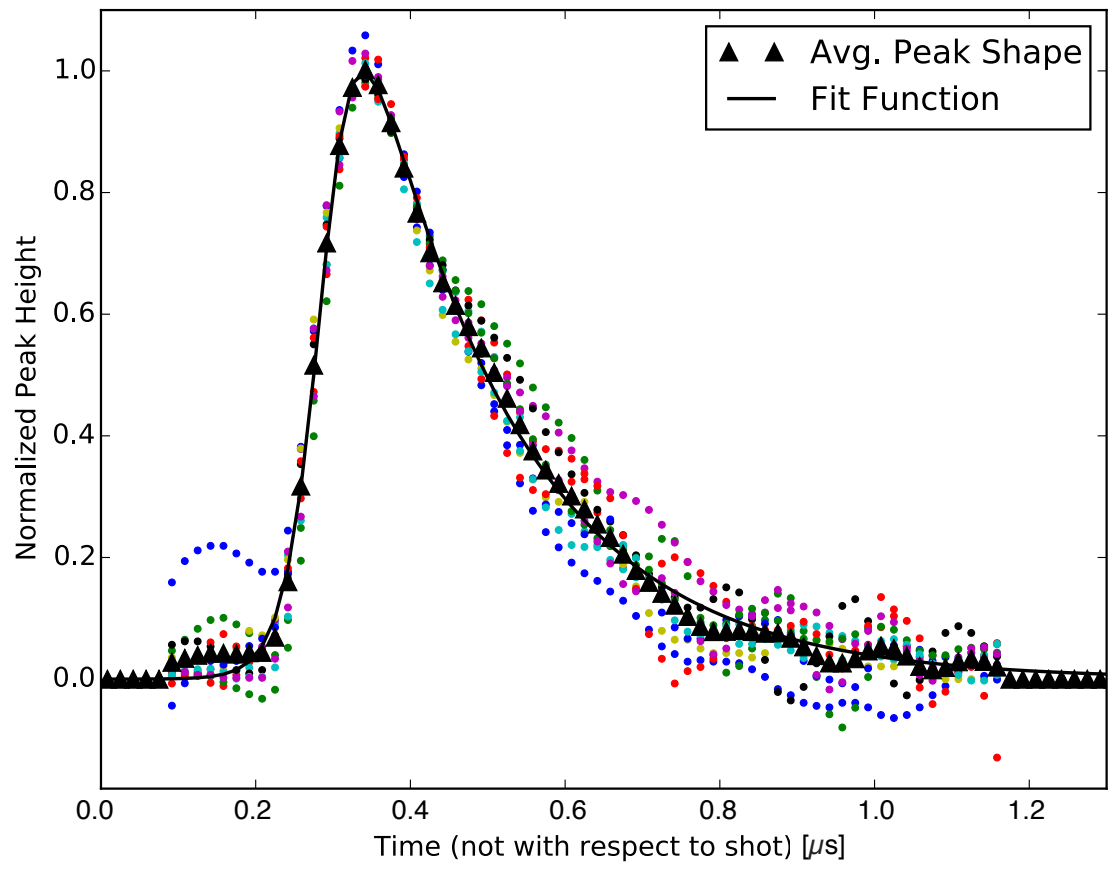

Figure 5.9: The individual data points (circles) for the sample peaks and the average peak shape (black triangles) are plotted along with the fit function (black line). Note that the time interval displayed is not with respect to the beginning of the plasma shot. The normalized amplitude height of each peak is aligned.

distinguish between real events and background noise. Each control file contains the set of time intervals for sample peaks in a data file. The resulting fitted data include the original peak height value, $\mathrm{V}$, the time at which that peak value occurs, $\mathrm{t}(\mathrm{ms})$, the amplitude of the fitted pulse-shape function, $\mathrm{A}$, and the uncertainty in the fit, $\sigma \mathrm{A}$.

Inputs to the Analysis Code There are several inputs for the first analysis code. The code, written in Python, fits a pulse-shape function to the raw data. A master file contains relevant information for each raw data file. A separate configuration file, called Signal Analysis Configuration File, has settings for formatting saved data and plotting the fitted data. The control file contains relevant parameters that are read by the analysis codes. It contains information such as the number of the plasma discharge (shot), the length of time 


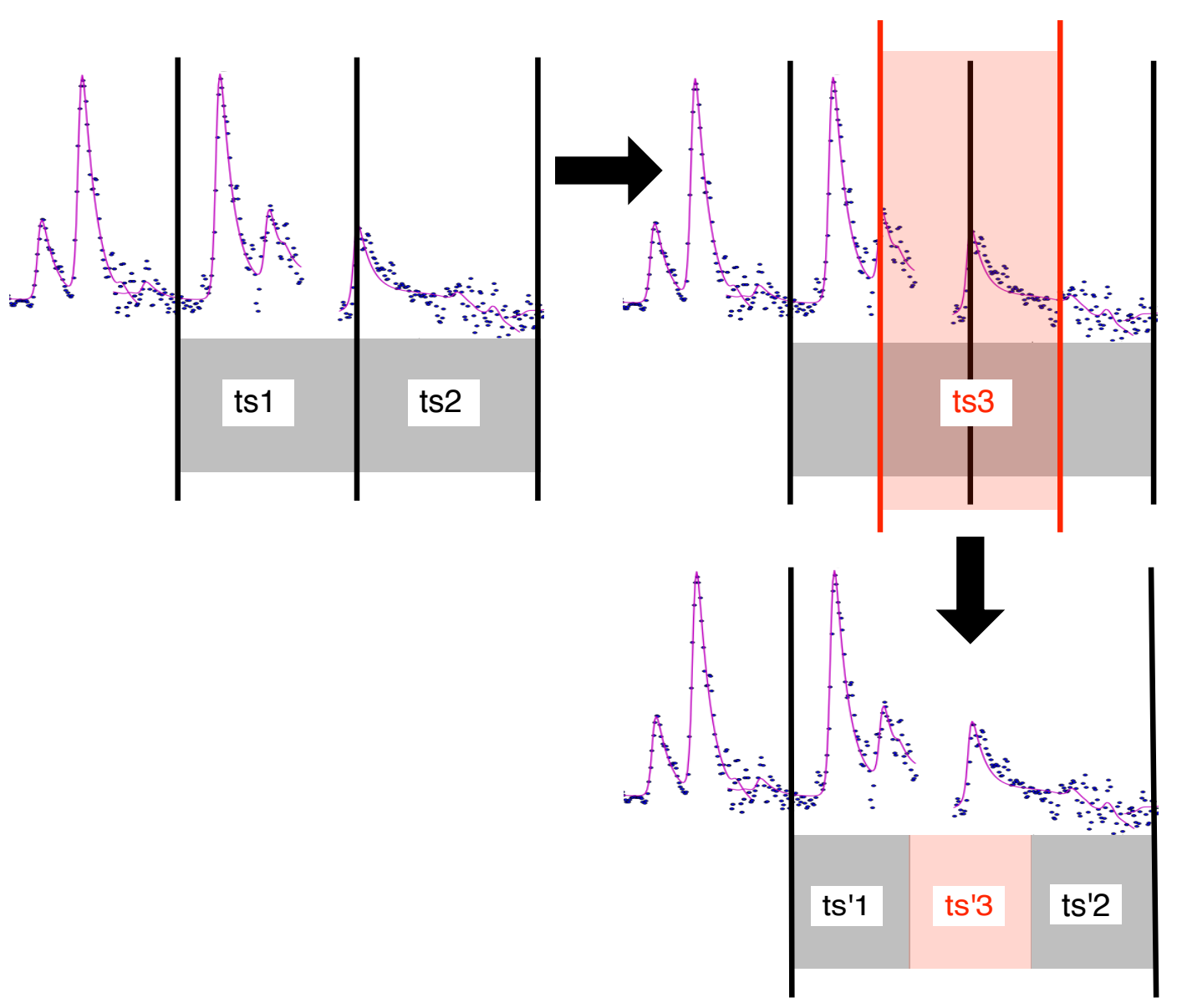

Figure 5.10: The raw data file is split into finite time intervals. The first analysis code iteratively fits data in these finite time slices (shown in gray). After the initial fitting, new finite time intervals (slices shown in red) are chosen such that they span the edges of the first set of time intervals. Finally, the central region of each fitted time interval (gray and red) is adjoined to create one file of continuously fitted data.

of the shot to be analyzed, the raw data file name, the raw data file directory, the channel number, and time intervals for a set of sample peaks. Each channel for every shot has a unique control file used as an input to the analysis code.

After formatting the appropriate input files with relevant parameters, a set of sample peaks for an individual channel are chosen. The time intervals for these peaks are added to 
the input files. Then, the first analysis code is run which finds potential particle signals in the data and uses a function to fit the signals. An output data file is written which contains the fit amplitudes of these potential particle signals.

\subsection{Second Step: Create Energy Spectra and Production Rates}

The second analysis step, highlighted in green in Figure 5.1, determines particle energy spectra from fitted data. The energy spectra are then integrated to calculate particle production rates.

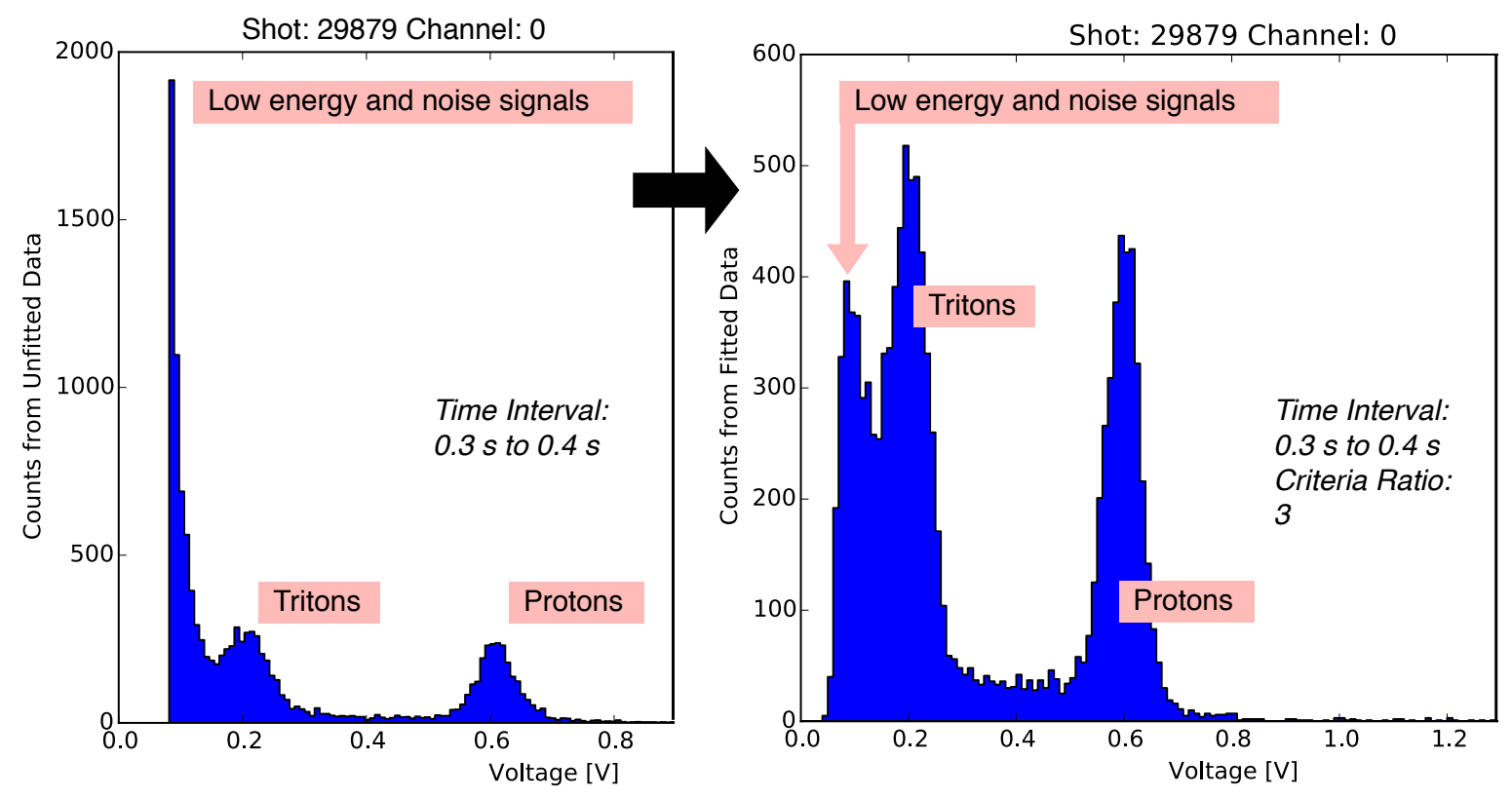

Figure 5.11: The image to the left bins all of the raw (unfitted) data by signal height. Binning fitted data by pulse amplitude and using a criteria of merit ratio is displayed in the image to the right. This reduces the lower energy and noise signals contribution to the energy spectra.

Criteria of Merit Value A criteria of merit, called CR, is used to reduce the contribution of low energy and background noise to the energy spectra,

$$
C R=\frac{\sigma A}{A}
$$


where $\mathrm{A}$ is the fit amplitude (fitted peak height of the pulse in the raw data) and $\sigma \mathrm{A}$ is the uncertainty in the fit amplitude. The CR ratio is used as a figure of merit when deciding whether or not to use a value from the fitted data file when creating an energy spectrum. As seen in Figure 5.11, the ratio has the greatest effect on amplitude values less than 300 $\mathrm{mV}$ (within the triton peak range). The largest uncertainty in a fit occur with low energy and noise signals. Therefore, the larger the CR parameter the more low energy and noise signals are histogrammed and the lower the CR parameter the less low energy and noise signals are histogrammed. The ratio is individually chosen for each channel to suppress noise while avoiding excluding real events, which results in a distribution where fusion tritons and protons are distinguishable from low energy and noise signals.[55] Once a CR value is chosen, it is saved in the corresponding control file for the channel.

Unfortunately, the triton events in the energy spectra are within the range of low energy and noise signals. Therefore, it can be difficult to clearly distinguish between where the low energy and noise signals end and where the triton signals begin. A crude minimum cutoff value is not used because it would mean a loss of potentially useful data regarding triton events. Therefore, the optimal CR value is chosen such that it is possible to distinguish between the triton signals and the low energy and noise signals. Figure 5.12 displays an example of an appropriate CR value; the histograms in Figure 5.12 have $100 \mathrm{~ms}$ time intervals.

Triton Spectra Though this work focuses on proton data, future analysis work will involve extracting triton signals after the CR has been applied. This future analysis will require a careful study of the shape of the distribution of small signals and the development of a fitting procedure to extract the triton contribution from the background. There are two main motivations for resolving the triton energy spectra: 1) combining triton and proton production rates could double the amount of data available for every plasma pulse 

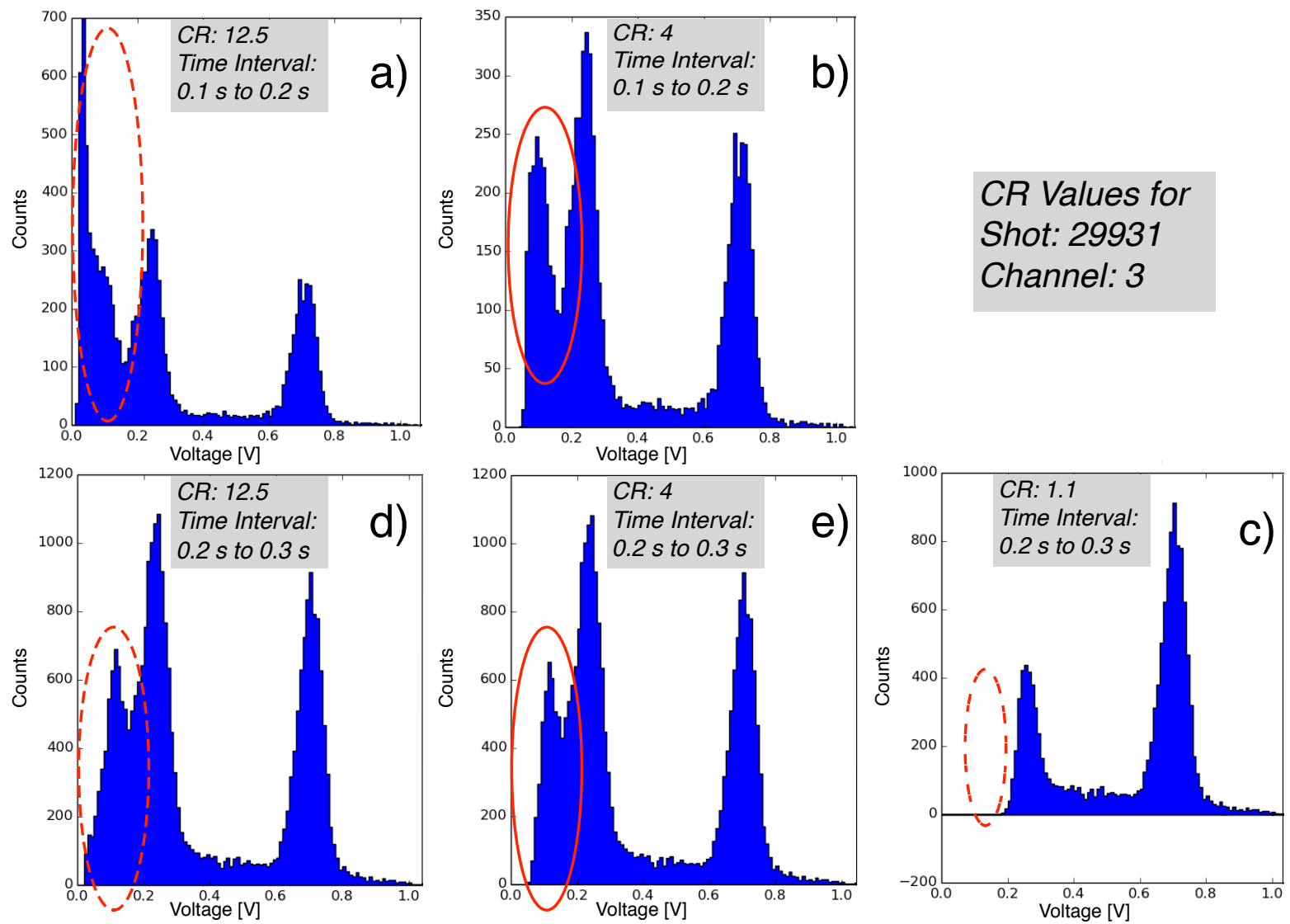

Figure 5.12: Above are histograms of pulse amplitudes for $100 \mathrm{~ms}$ time intervals using different $C R$ values. Figure b) and e) use a CR value of 4 for Shot 29931, Channel 3. This is an appropriate value because it distinguishes between the triton signals and low energy and noise signals in a bulk of the histograms for $100 \mathrm{~ms}$ time intervals. Figure a) and d) use a CR value of 12.5, which is too high of a value because an unnecessary amount of low energy and noise signals ( $0 \mathrm{~V}$ to $0.1 \mathrm{~V}$ ) become included in the histogram. Figure c) uses a $\mathrm{CR}$ value of 1.1 which is too low of a value because not enough low energy and noise signals are histogrammed.

and 2) differences between triton and proton energy spectra and rates could offer insight into the anisotropy of DD fusion reactions. If there were no anisotropy in the DD fusion reactions, then the products would be emitted uniformly from the plasma. Therefore a lack of anisotropy would lead to almost identical energy spectra and rates for both protons and tritons. Preferential directions of DD charged fusion product emission could influence these energy spectra and rates. 


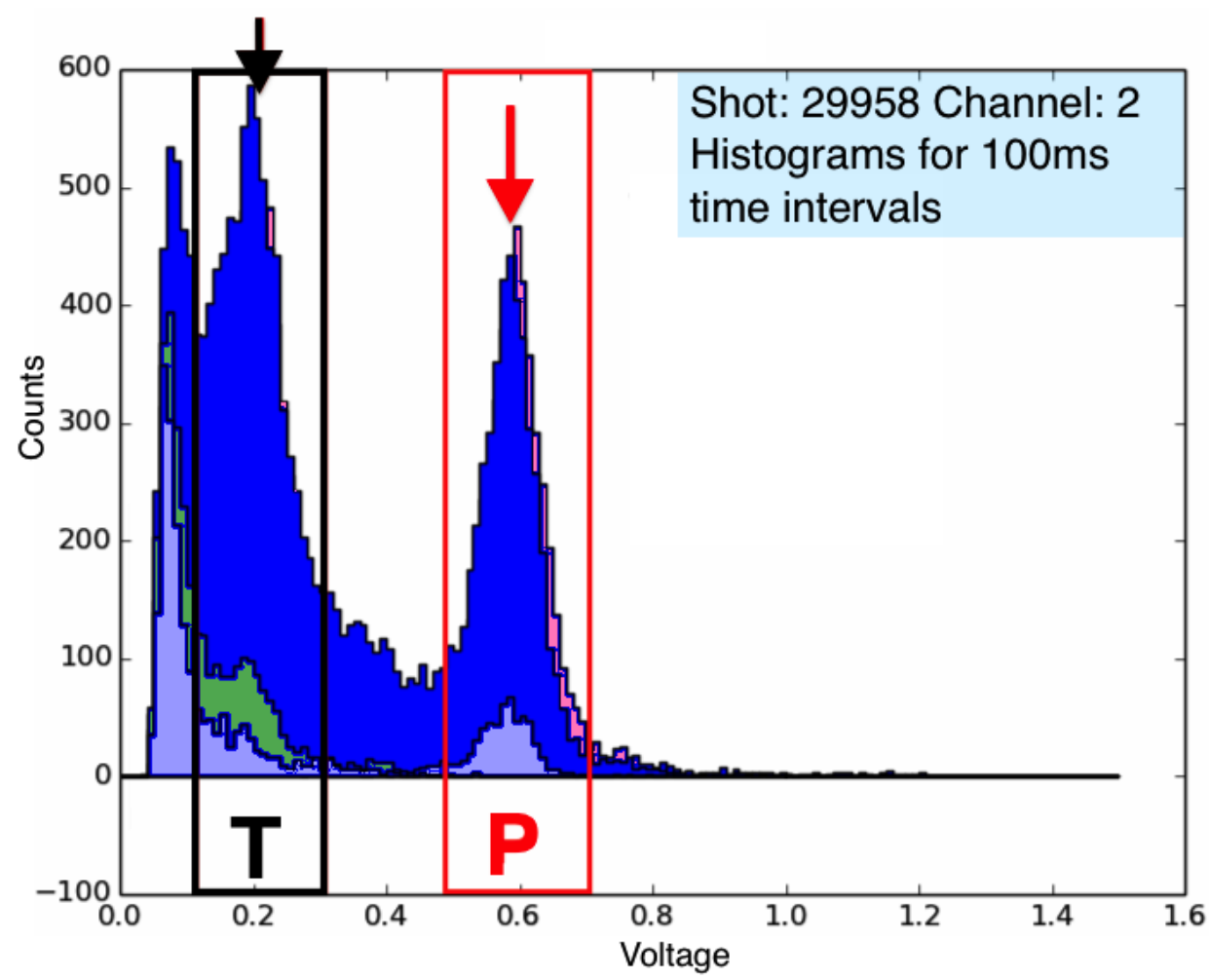

Figure 5.13: Proton and triton energy spectra are plotted above. The proton peaks are in the red box to the right. The red box ranges from the minimum to maximum proton peak values. The triton peaks are in the black box to the left. The arrows point to the mean values of the triton and proton peaks. For this example the control file parameter values used are: $p_{\min }=0.5 \mathrm{~V}, p_{\max }=0.7$ $\mathrm{V}, p_{\text {mean }}=0.6 \mathrm{~V}, T_{\text {min }}=0.13 \mathrm{~V}, T_{\text {max }}=0.31 \mathrm{~V}$, and $T_{\text {mean }}=0.21 \mathrm{~V}$.

Binning the Fitted Data Peak amplitudes from the fitted data are binned to create a histogram. Because the particle pulse heights are directly proportional to the incident particle energy on the detector, histogramming the pulse amplitudes (V) creates an energy spectrum for that channel. The length of the time intervals for which a histogram is created is specified in the control file. For example, if the time interval parameter is equal to $100 \mathrm{~ms}$, the algorithm will histogram pulse amplitudes in the first $100 \mathrm{~ms}$ of the fitted data file. The algorithm will then histogram the next $100 \mathrm{~ms}$ in the data file, and so on. The process will continue until histograms have been made for every consecutive $100 \mathrm{~ms}$ time interval in 
the data file. The standard data file contains around $0.5 \mathrm{~s}$ of data. Therefore typically five histograms will be created for one channel when using a $100 \mathrm{~ms}$ time interval. An example of these histograms plotted on top of each other can be seen in Figure 5.13.

The proton peak heights generally range from $0.5 \mathrm{~V}$ to $0.8 \mathrm{~V}$. The peak height is defined by the initial signal size, signal attenuation, and the settings of the shaping amplifier used in data collection. The shaping amplifier integration and differentiation time constants change the signal: amplitude, width, rise time, and fall time. Using smaller shaping time constants can create a faster signal (smaller widths) with a smaller amplitude while larger shaping constants create a slower signal with larger amplitudes. To resolve pulses above the electrical background noise, shaping time constants of $100 \mathrm{~ns}$, for both integration and differentiation, were used.

The triton peak heights fall within a range of $0.2 \mathrm{~V}$ to $0.4 \mathrm{~V}$. These values are close to low energy and noise signals. Therefore, depending on the channel and shot, the triton peaks will not be completely separated from low energy and noise signals like the proton peaks. Figure 5.13 shows proton peak signal heights in the red box to the right. The triton peak signal heights are in the black box to the left. The arrows point to the mean values of the triton and proton peaks. The minimum, maximum, and mean values for protons and tritons are chosen and saved as parameters in the control file. Therefore, these values are used as input parameters for the analysis code and this second procedure.

Helium-3 ions cannot be resolved in the data because the majority of their signal height range $(0.18 \mathrm{~V}$ to $0.24 \mathrm{~V})$ is within the noise levels. If steps can be taken to mitigate noise in the next instrument design, an analysis of these ions might be possible. 
Creating Particle Production Rates After the histograms (energy spectra) for each time interval have been determined each histogram is then integrated over the range of proton peaks to obtain the total number of protons detected during the corresponding time interval to calculate the corresponding rate.

An example of a proton production rate as a function of time is shown in Figure 5.14. In this figure, there is one data point for each histogram. Figure 5.15 displays a proton rate production plot which uses a $1 \mathrm{~ms}$ time interval as opposed to $100 \mathrm{~ms}$. Note that an individual histogram is associated with each data point. Therefore, a proton rate production plot which uses $100 \mathrm{~ms}$ time intervals would only have five data points.

In order to determine integrations limits, $50 \mathrm{~ms}$ to $100 \mathrm{~ms}$ time intervals have been used to have enough statistics in each channel. The extracted proton rates have consequently very coarse time resolution. In order to detect variations of the order of a few ms the time intervals for histogramming has been reduced to typically $1 \mathrm{~ms}$. The integration limits used were those determined previously using the high statistics histograms. 


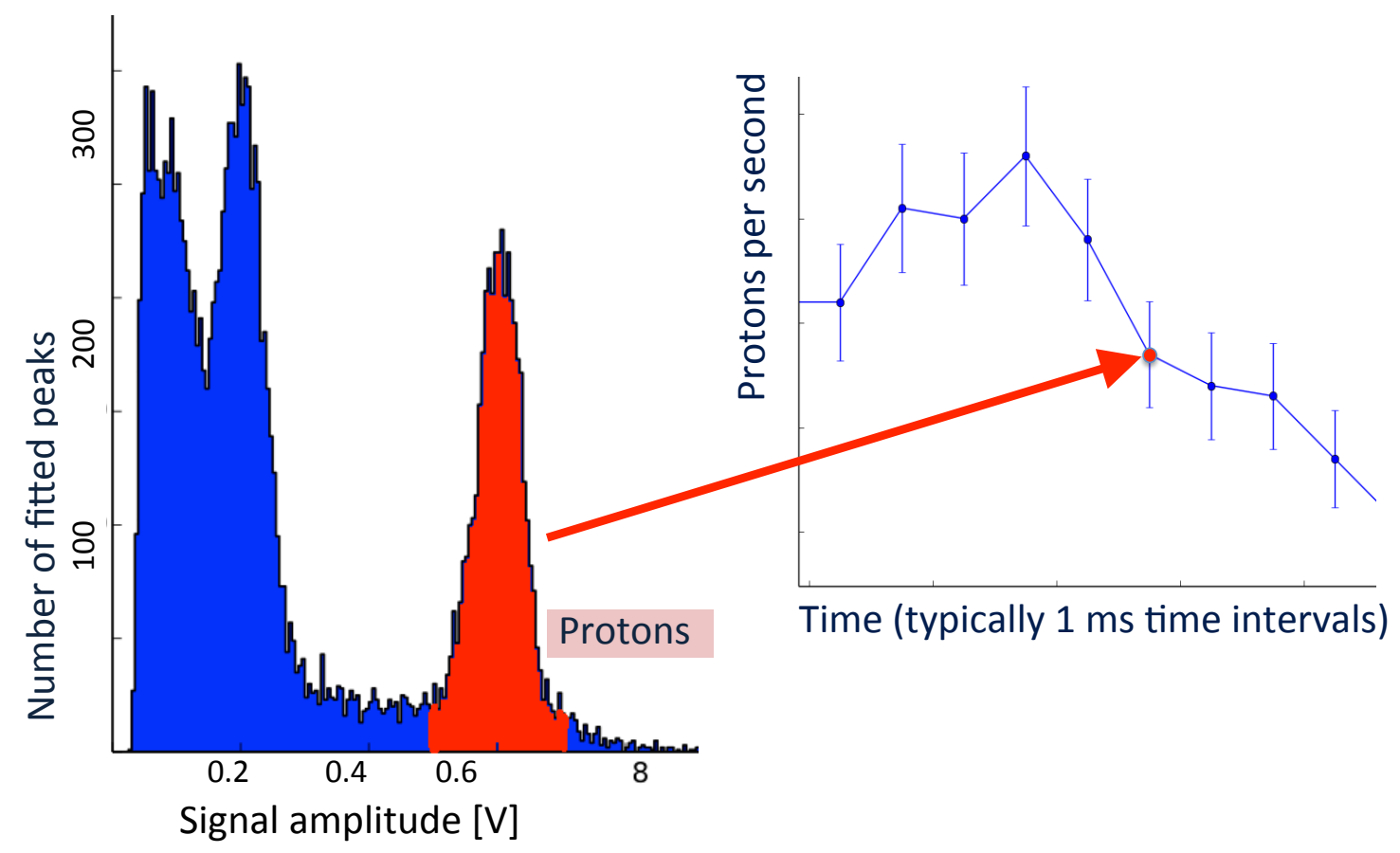

Figure 5.14: The above image is qualitative. Integrating signals in the proton energy spectra yields the total number of protons detected in a finite time interval, $\Delta \mathrm{t}$. The ratio of the proton sum and $\Delta \mathrm{t}$ corresponding to the histogram is plotted as a rate value. A collection of these data points (from a collection of histograms) creates an emission rate of protons during a plasma discharge. 


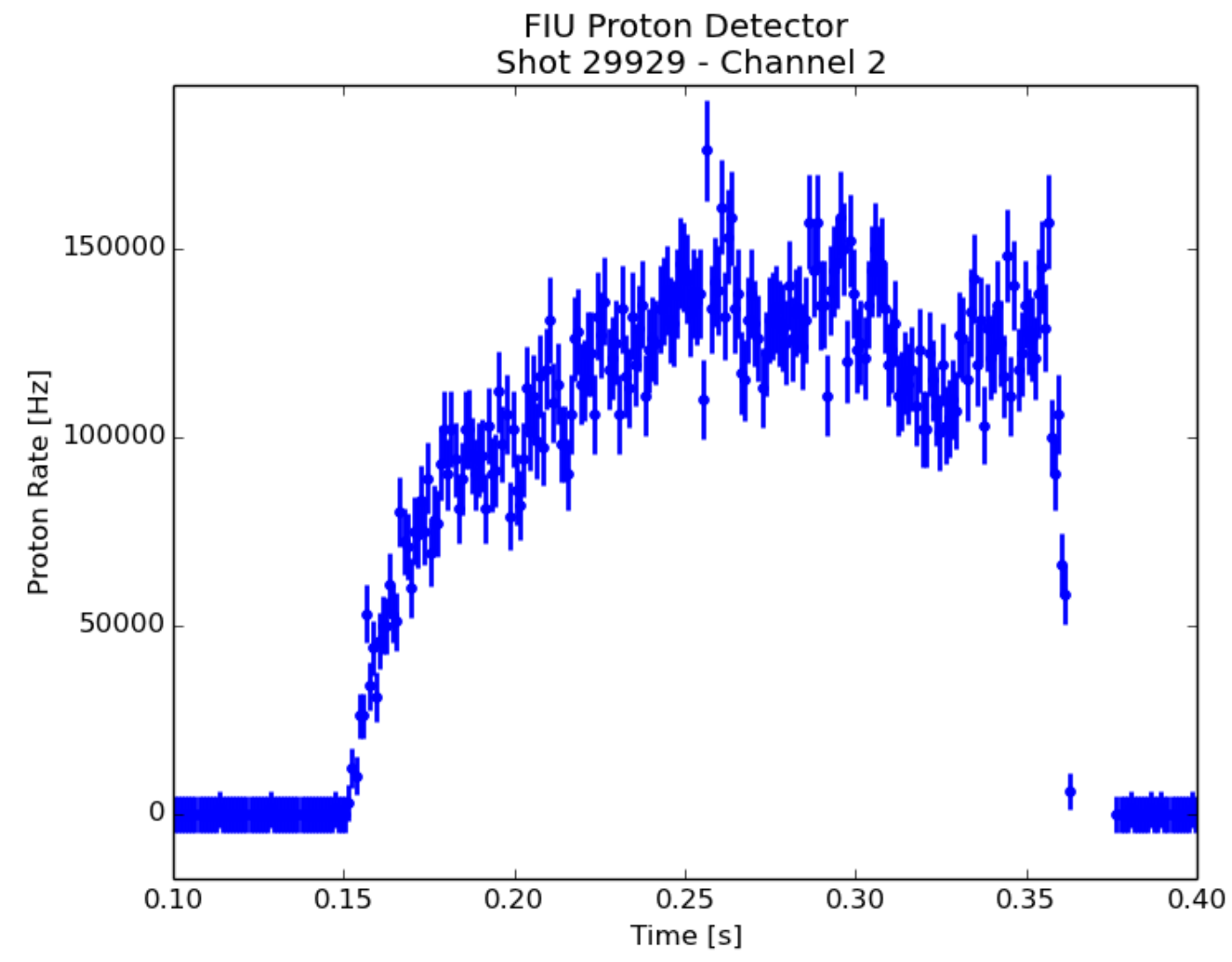

Figure 5.15: A proton production rate plot, where each point represents a sum taken over successive histograms of $1 \mathrm{~ms}$ time intervals. 


\subsection{Mid-plane Radii}

One of the ultimate goals of the PD instrument, and potential new versions of the instrument, is the determination of the proton production profile in the poloidal plane. It is customary to characterize the central orbits associated with each detector-collimator by its mid-plane intersection radius. The local proton production rate is expected to be maximum at the magnetic axis, and a detector whose mid-plane crossing radius coincides with the magnetic axis should have the largest signal. This typically happens closest to the plasma core. When the poloidal trajectory for particles entering one channel of the instrument is plotted, the radial value along the mid-plane or equatorial plane where $\mathrm{Z}=0$, is the mid-plane radius (Figure 5.16). This mid-plane radius value is calculated from orbit code trajectories, which use the equilibrium magnetic field configuration determined by the EFIT code. Additionally, as discussed in Chapter 2, there is a thickness associated with the bundle of orbits that can enter a detector. Therefore, the mid-plane radii values have an uncertainty of $+/-3 \mathrm{~cm}$. 


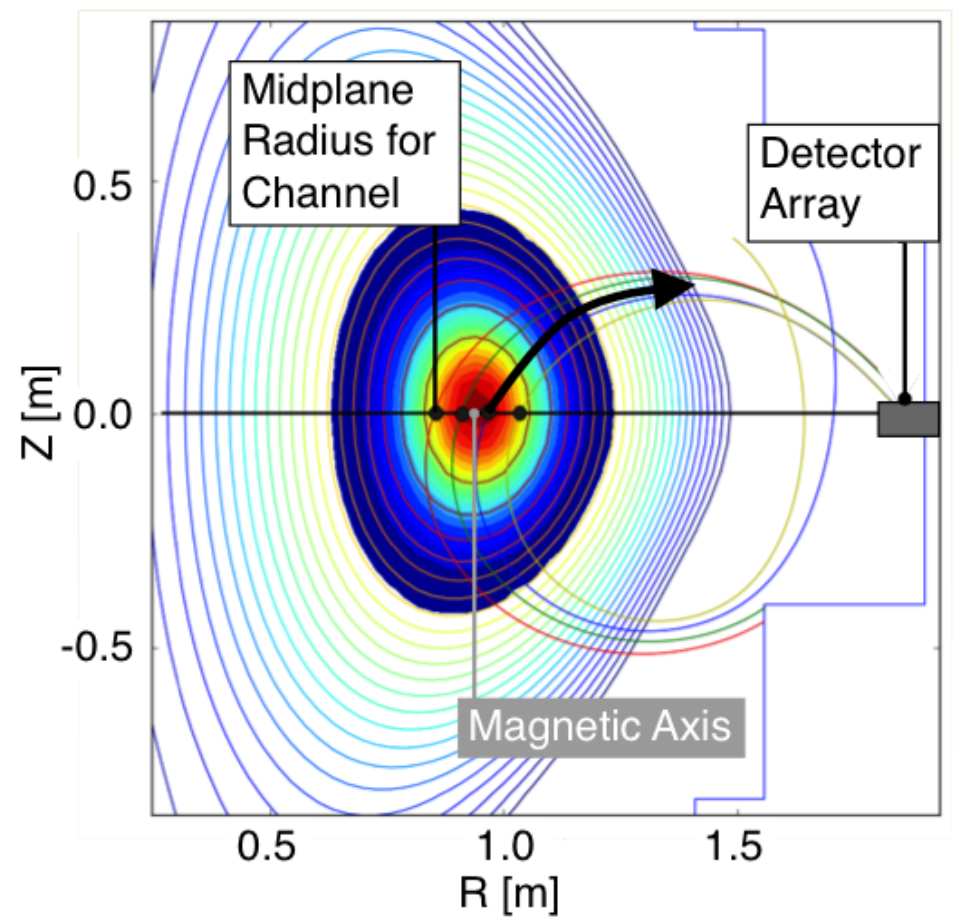

Figure 5.16: The above image is qualitative. The above figure displays the characteristic mid-plane radii (the $\mathrm{R}$ value when $\mathrm{Z}=0$ ) for the four $\mathrm{PD}$ channels as well as the magnetic axis. 


\section{CHAPTER 6}

\section{RESULTS AND DISCUSSION}

This chapter discusses fast ion relaxation times, proton production rates, and proton emission profiles during plasma oscillations. ${ }^{1}$

\subsection{Ion Relaxation Times}

When beam atoms are injected into the plasma, they ionize and transfer momentum to plasma electrons and ions through Coulomb collisions. During momentum transfer, the beam ions slow down from their initial kinetic energy to thermal energy, energy of the background plasma ions. The momentum transfer is mainly in the direction anti-parallel to the initial velocity of the beam ion. For fully ionized plasmas, collisions between charged particles (as opposed to collisions between charged particles and neutral particles) are dominant.

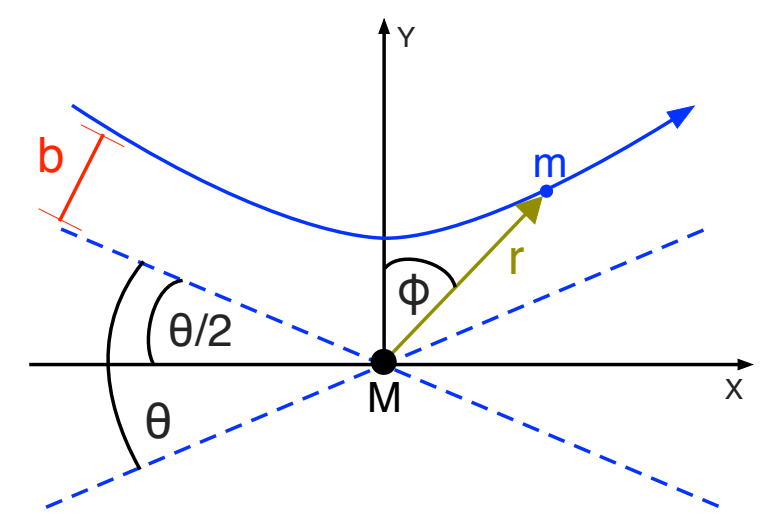

Figure 6.1: Above is a general image for Rutherford scattering where $b$ is the distance of closest approach of $m$ to M.[66] Note that if $m$ is the electron mass and $\mathrm{M}$ is an ion of positive charge, then $\mathrm{m}$ would deflect towards the ion.

\footnotetext{
${ }^{1}$ I recognize the CCFE MAST Data Access Policy which grants me the use of MAST data in my dissertation with a statement that the MAST data used (excluding cited published data) is given preliminary status.
} 
Effective Coulomb Cross Section The effective cross section for the non-relativistic elastic scattering of an electron off of an ion in the plasma (6.4), is found using the hard sphere cross section (6.1) and the particle's potential energy (Coulomb potential) and kinetic energy during a collision (6.2). Note that the distance between the electron and ion, $\mathrm{r}$, is evaluated at $\mathrm{b}$, the distance of closest approach of the electron to the ion before it is scattered or deflected.[3][65]

$$
\begin{gathered}
\sigma=\pi r^{2} \\
\frac{Z e^{2}}{4 \pi \epsilon_{0} b}=\frac{m v^{2}}{2} \\
b=\frac{2 Z e^{2}}{4 \pi \epsilon_{0} m v^{2}} \\
\left.\sigma\right|_{r=b}=\frac{\pi Z^{2} e^{4}}{\left(4 \pi \epsilon_{0}\right)^{2}} \frac{2^{2}}{\left(m v^{2}\right)^{2}}=\frac{Z^{2} e^{4}}{4 \pi \epsilon_{0}^{2} m^{2} v^{4}}
\end{gathered}
$$

Large Angle Coulomb Cross Section A second expression for the cross section for Coulomb scattering is based on Rutherford scattering as displayed in Figure 6.1. The electron of mass $\mathrm{m}$ is deflected by the Coulomb force of the large target ion, mass M. To solve for the impact parameter $b$ as a function of scattering angle, the conservation of angular momentum and linear momentum are used from (6.5) to (6.8).

$$
\begin{gathered}
\vec{L}=\vec{r} \times \vec{p}=I \vec{\omega} \rightarrow m v b=m r^{2}\left(\frac{d \phi}{d t}\right) \rightarrow d t=\frac{r^{2} d \phi}{v b} \\
d \vec{p}=\int \vec{F}_{\text {Coulomb }} d t \rightarrow \Delta P_{Y}=\int \frac{Z e^{2} \cos \phi}{4 \pi \epsilon_{0} r^{2}} d t \\
\Delta P_{Y}=\int_{-(\pi / 2-\theta / 2)}^{(\pi / 2-\theta / 2)} \frac{Z e^{2} \cos \phi}{4 \pi \epsilon_{0} r^{2}}\left(\frac{r^{2} d \phi}{v b}\right)=\frac{Z e^{2} 2 \cos (\theta / 2)}{4 \pi \epsilon_{0} v b} \\
\vec{L}=\vec{r} \times \vec{p} \rightarrow \Delta P_{Y}=m v \sin (\theta / 2)-(-m v \sin (\theta / 2))=2 m v \sin (\theta / 2)
\end{gathered}
$$


Setting (6.7) equal to $(6.8)^{1}$ results in a new expression for the impact parameter $b$ in (6.9).

$$
\begin{gathered}
b=\frac{Z e^{2} \cot (\theta / 2)}{4 \pi \epsilon_{0} m v^{2}} \text { where }\left.b\right|_{\theta=\pi / 2}=\frac{Z e^{2}}{4 \pi \epsilon_{0} m v^{2}}=b_{0} \\
\left.\sigma\right|_{\theta=\pi / 2}=\frac{Z^{2} e^{4}}{16 \pi \epsilon_{0}^{2} m^{2} v^{4}}
\end{gathered}
$$

Therefore a new expression for the cross section of Coulomb scattering of an electron off of an ion for large angles is found in (6.10).[66] The value of the large angle Coulomb $\sigma$ is smaller than the effective $\sigma$ by a factor of four.

Small Angle Coulomb Cross Section To find the $\sigma$ for small angle scattering, the rate of change of parallel and perpendicular components of the velocity's average deviation and variance are needed. The magnitude of the perpendicular component of the velocity $\Delta \overrightarrow{v_{\perp}}$ (perpendicular with respect to the magnetic field) is smaller than the parallel component of the velocity $\Delta \overrightarrow{v_{\|}}$. This means as electrons slow down and change velocity, they retain their original direction and scattering between electrons and target ions is minimized.

An expression for theta $(6.12)^{2}$ in terms of $b$, from (6.9), is used to express the perpendicular component of the variance of the velocity.[65]

$$
\begin{gathered}
\tan \frac{\theta}{2}=\frac{Z e^{2}}{4 \pi \epsilon_{0} m v^{2}} \frac{1}{b}=\frac{b_{0}}{b} \\
\sin \theta=\frac{2 \cot (\theta / 2)}{1+\cot ^{2}(\theta / 2)}=\frac{2\left(b / b_{0}\right)}{1+\left(b / b_{0}\right)^{2}} \\
\left(\Delta v_{\perp}\right)^{2}=(v \sin \theta)^{2}=v^{2}\left(\frac{2\left(b / b_{0}\right)}{1+\left(b / b_{0}\right)^{2}}\right)^{2}
\end{gathered}
$$

For an electron passing a distance $v d t$ (in the direction of its initial velocity) through a scattering volume element $2 \pi b d b v d t$, it will experience small changes perpendicular to the

\footnotetext{
${ }^{1}$ The trigonometric identity, $\sin (A-B)=\sin A \cos B-\cos A \sin B$, is used.

${ }^{2}$ The trigonometric identities used are: $\sin A=2 \sin (A / 2) \cos (A / 2), \tan A=\sin A / \cos A, \csc A=\sin A^{-1}, \cot A=$ $\tan A^{-1}$, and $(\csc A)^{2}=1+(\cot A)^{2}$.
} 
direction of its initial velocity. The distance traveled and the scattering volume element can be used to find the average of the perpendicular component of the variance of the velocity.[65]

$$
\begin{gathered}
\frac{d\left\langle\left(\Delta v_{\perp}\right)^{2}\right\rangle}{d t}=\int(v \sin \theta)^{2}(n 2 \pi b d b v)=\int \frac{4 v^{2}\left(b / b_{0}\right)^{2}}{\left(1+\left(b / b_{0}\right)^{2}\right)^{2}}(n 2 \pi b d b v) \\
\text { let } A_{0}=8 \pi n v^{3} \quad A_{1}=\frac{1}{b_{0}} \quad x=b \quad d x=d b \\
\frac{d\left\langle\left(\Delta v_{\perp}\right)^{2}\right\rangle}{d t}=A_{0} \int \frac{A_{1}^{2} x^{3} d x}{\left(1+A_{1}^{2} x^{2}\right)^{2}} \\
\frac{d\left\langle\left(\Delta v_{\perp}\right)^{2}\right\rangle}{d t}=\frac{A_{0}}{2 A_{1}{ }^{2}}\left[\frac{1}{1+A_{1}{ }^{2} x^{2}}+\left.\ln \left(1+A_{1}{ }^{2} x^{2}\right)\right|_{b=0} ^{b=b_{\max }}\right.
\end{gathered}
$$

The upper limit used for the impact parameter in (6.17) is $b_{\max }$ because the integrand approaches infinity as b approaches infinity.

$$
\begin{gathered}
\frac{d\left\langle\left(\Delta v_{\perp}\right)^{2}\right\rangle}{d t}=\frac{A_{0}}{2 A_{1}^{2}}\left[-1+\frac{1}{1+\left(b_{\max } / b_{0}\right)^{2}}+\ln \left(1+\left(b_{\max } / b_{0}\right)^{2}\right)\right] \\
\text { let } \Lambda=\frac{b_{\max }}{b_{0}} \text { where } \Lambda>>1 \\
\frac{d\left\langle\left(\Delta v_{\perp}\right)^{2}\right\rangle}{d t} \simeq 4 \pi n v^{3} b_{0}{ }^{2}\left(\ln \left(\Lambda^{2}\right)\right)=\frac{n Z^{2} e^{4} \ln \Lambda}{2 \pi \epsilon_{0}{ }^{2} m^{2} v}
\end{gathered}
$$

Note that $\ln \Lambda$ is usually referred to as the Coulomb logarithm. Equation (6.20) represents the rate of change in time of the average of the perpendicular component of the variance of the velocity. Namely, scattering occurs when the direction of the velocity (with respect to the magnetic field) changes from its initial velocity direction.

Conservation of energy can then be used in (6.21) to find the average of the deviation of the parallel component of the velocity in terms of the average of the perpendicular 
component of the variance of the velocity.

$$
\begin{gathered}
v_{1}=v=\left(v_{\|}+v_{\perp}\right)=v_{\|} \text {where } v_{\perp}=0 \\
v_{2}=v_{2 \|}+v_{2 \perp}=\left(v_{\|}+\Delta v_{\|}\right)+\left(v_{\perp}+\Delta v_{\perp}\right)=\left(v_{\|}+\Delta v_{\|}\right)+\left(\Delta v_{\perp}\right)
\end{gathered}
$$

Using symmetry in the isotropic scattering of electrons, in which electrons do not have a preferential direction of scattering in a uniform ion target, results in an average of the deviation in the perpendicular velocity equal to zero, $\left\langle\Delta v_{\perp}\right\rangle=0$.[65]

$$
\langle\Delta v\rangle=\left(\left\langle\Delta v_{\|}\right\rangle+\left\langle\Delta v_{\perp}\right\rangle\right)=\left\langle\Delta v_{\|}\right\rangle \text {where }\left\langle\Delta v_{\perp}\right\rangle=0
$$

The mean square deflections or average of the variance for the perpendicular component of the velocity, however, is not zero. Though for the approximation of incident electrons with a velocity greater than the target ion velocity, the average of the variance for the parallel component of the velocity can be approximated to zero.

$$
\begin{gathered}
\left\langle(\Delta v)^{2}\right\rangle=\left\langle\left(\Delta v_{\|}\right)^{2}\right\rangle+\left\langle\left(\Delta v_{\perp}\right)^{2}\right\rangle=\left\langle\left(\Delta v_{\perp}\right)^{2}\right\rangle \\
\text { where }\left\langle\left(\Delta v_{\|}\right)^{2}\right\rangle \rightarrow 0 \text { for } \frac{v_{\text {incident }}}{v_{\text {target }}}>>1
\end{gathered}
$$

The conservation of energy and Equation (6.21) through Equation (6.25) then yield an expression for the deviation in the parallel component of the velocity.

$$
\begin{gathered}
\frac{m v_{1}^{2}}{2}=\frac{m v_{2}^{2}}{2} \rightarrow \frac{m v^{2}}{2}=\frac{m\left(v_{\|}+\Delta v_{\|}\right)^{2}}{2}+\frac{m\left(\Delta v_{\perp}\right)^{2}}{2} \\
v^{2}=\left(v+\Delta v_{\|}\right)^{2}+\left(\Delta v_{\perp}\right)^{2}=v^{2}+\left(\Delta v_{\|}\right)^{2}+2 v \Delta v_{\|}+\left(\Delta v_{\perp}\right)^{2} \\
0=\left(\Delta v_{\|}\right)^{2}+2 v \Delta v_{\|}+\left(\Delta v_{\perp}\right)^{2}=\left\langle\left(\Delta v_{\|}\right)^{2}\right\rangle+2 v\left\langle\Delta v_{\|}\right\rangle+\left\langle\left(\Delta v_{\perp}\right)^{2}\right\rangle
\end{gathered}
$$




$$
\left\langle\Delta v_{\|}\right\rangle=\frac{-1}{2 v}\left\langle\left(\Delta v_{\perp}\right)^{2}\right\rangle
$$

The rate of change in the deviation of the parallel component of the velocity can be related to the rate of change in the variance of the perpendicular component of the velocity through using (6.20) and (6.29).[65]

$$
\frac{d\left\langle\Delta v_{\|}\right\rangle}{d t}=\frac{-1}{2 v} \frac{d\left\langle\left(\Delta v_{\perp}\right)^{2}\right\rangle}{d t}=\frac{-n Z^{2} e^{4} \ln \Lambda}{4 \pi \epsilon_{0}^{2} m^{2} v^{2}}
$$

Equation (6.30) represents the rate of loss of momentum in the direction of the electron's initial velocity.

The rate of the loss of momentum is related to the collision frequency (6.31) and collision time (6.32).[58][65]

$$
\begin{gathered}
\nu_{c}=\frac{-1}{v} \frac{d\left\langle\Delta v_{\|}\right\rangle}{d t}=\frac{n Z^{2} e^{4} \ln \Lambda}{4 \pi \epsilon_{0}^{2} m^{2} v^{3}} \\
\tau_{c}=\frac{1}{\nu_{c}}=\frac{4 \pi \epsilon_{0}^{2} m^{2} v^{3}}{n Z^{2} e^{4} \ln \Lambda} \\
\sigma=\frac{\nu_{c}}{n v}=\frac{Z^{2} e^{4} \ln \Lambda}{4 \pi \epsilon_{0}^{2} m^{2} v^{4}}
\end{gathered}
$$

Therefore, the cross section for Coulomb scattering that takes into account the cumulative effect of small angle scattering is expressed in (6.33).[65]. The small angle scattering $\sigma$ is greater than the large angle scattering $\sigma$ by a factor of $4 \ln \Lambda$ and greater than the effective $\sigma$ by a factor of $\ln \Lambda$. Small angle Coulomb scattering happens more often owing to the $r^{-2}$ nature of the Coulomb force.[65]

Slowing Down Time The characteristic ion slowing down time $\tau_{S}$ is the amount of time it takes for beam ions to thermalize. When beam ions thermalize, they decelerate and their initial kinetic energy decreases to the thermal energy of the background plasma ions. 
The previous expressions for Coulomb scattering and collision frequency and collision times can be applied to ions. Similarly, as for electrons in (6.32), the slowing down time of ions because of Coulomb collisions with electrons is proportional to the cube of the beam ion velocity, $v^{3}$.[69] Therefore the slowing down of beam ions because of Coulomb collisions with background plasma ions, or thermal ions, is proportional to the plasma temperature and density, $T^{3 / 2} / n$. Additionally, like the incident electrons previously discussed, beam ions lose momentum in the direction anti-parallel to the direction of their initial velocity.[65] In general the fast ion slowing down time can be described as the rate of change of velocity in the direction of the initial velocity.

$$
\tau_{S}=-v\left(\frac{d\left\langle\Delta v_{\|}\right\rangle}{d t}\right)^{-1}
$$

For MAST discharges, the value of $\tau_{S}$ is typically calculated using electron density and electron temperature measurements ${ }^{1}$ and is on the order of tens of milliseconds.[67]

For the below expressions, $n$ is the bulk ion density, $T$ is the bulk ion temperature, $\ln \Lambda$ is the Coulomb logarithm, and $\epsilon_{0}$ is the permittivity of free space.[58] In MAST plasmas, which are considered to be fully ionized, the approximations that $n$ is the same as the bulk electron density and $T$ is the same as the bulk electron temperature are used.[67]

$$
\begin{gathered}
\langle\nu\rangle=\frac{n Z^{2} e^{4} \ln \Lambda}{4 \pi \epsilon_{0}^{2} m^{2}}\left(\frac{m}{T}\right)^{3 / 2} A_{0} \text { where } v=\left(\frac{T}{m}\right)^{1 / 2} \\
\left\langle\nu_{e e}\right\rangle \simeq \frac{n_{e} e^{4} \ln \Lambda}{\epsilon_{0}^{2} m^{1 / 2} T^{3 / 2}} \quad \text { for } \quad A_{0}=\frac{4 \pi n_{e}}{n} \\
\left\langle\nu_{e i}\right\rangle=\frac{n 2^{1 / 2} Z^{2} e^{4} \ln \Lambda}{12 \pi^{3 / 2} \epsilon_{0}^{2} m^{1 / 2} T^{3 / 2}} \text { for } A_{0}=\frac{2^{1 / 2}}{3 \pi^{1 / 2}} \\
\tau_{e i}=\frac{12 \pi^{3 / 2} \epsilon_{0}^{2} m^{1 / 2} T^{3 / 2}}{n 2^{1 / 2} Z^{2} e^{4} \ln \Lambda}
\end{gathered}
$$

\footnotetext{
${ }^{1}$ These measurements are obtained from Thompson Scattering experiments.
} 


$$
\begin{gathered}
\left\langle\nu_{i i}\right\rangle=\frac{n Z^{4} e^{4} \ln \Lambda}{12 \pi^{3 / 2} \epsilon_{0}^{2} M^{1 / 2} T^{3 / 2}} \quad \text { for } \quad A_{0}=\frac{1}{3 \pi^{1 / 2}} \\
\tau_{i i}=\frac{12 \pi^{3 / 2} \epsilon_{0}^{2} M^{1 / 2} T^{3 / 2}}{n Z^{4} e^{4} \ln \Lambda}
\end{gathered}
$$

Note that the background plasma electrons and ions are assumed to have a a Maxwellian distribution when the NBI heating has ended.[69] The value of $A_{0}$ depends on spherical symmetry and integration factors resulting from the velocity space of the Maxwellian distribution of electrons in the plasma.[65][58]

Experimental Time Decay Constants The fast ion relaxation time $\tau_{R}$ is the amount of time it takes for the number of fast ions produced (after the neutral beam heating has discontinued) to slow down to the thermal energy of the plasma.[69] The experimental value (proportional to the slowing down time) will also be referred to as the time decay constant. Previous work with a neutral particle analyzer's experimental time decay constants, from $10 \mathrm{~ms}$ to $80 \mathrm{~ms}$, finds an almost one to one correlation between the measured time decay constants and the calculated slowing down time.[69] Time decay constants, however, above $30 \mathrm{~ms}$ diverged the most from calculated slowing down times. Additionally, it is noted that these time decay constants, as well as those measured with the PD, do not take into account the anisotropy of DD fusion reactions.[68]

In this present dissertation work, time decay constants are found for the DD neutron and DD proton rates. The proton rates, after the NBI has ended, are fit to an exponential function from which $\tau_{R}$ is extracted.

$$
\operatorname{Rate}(t)=R_{0} e^{-t / \tau} \rightarrow \ln (\text { Rate })=\left(\frac{-1}{\tau}\right) t+\ln \left(R_{0}\right)
$$

The value of $R_{0}$ is a constant equal to the rate at the time the NBI is turned off. The shaded regions of the proton production rate that is fit for one shot can be seen in Figure 6.2. In 


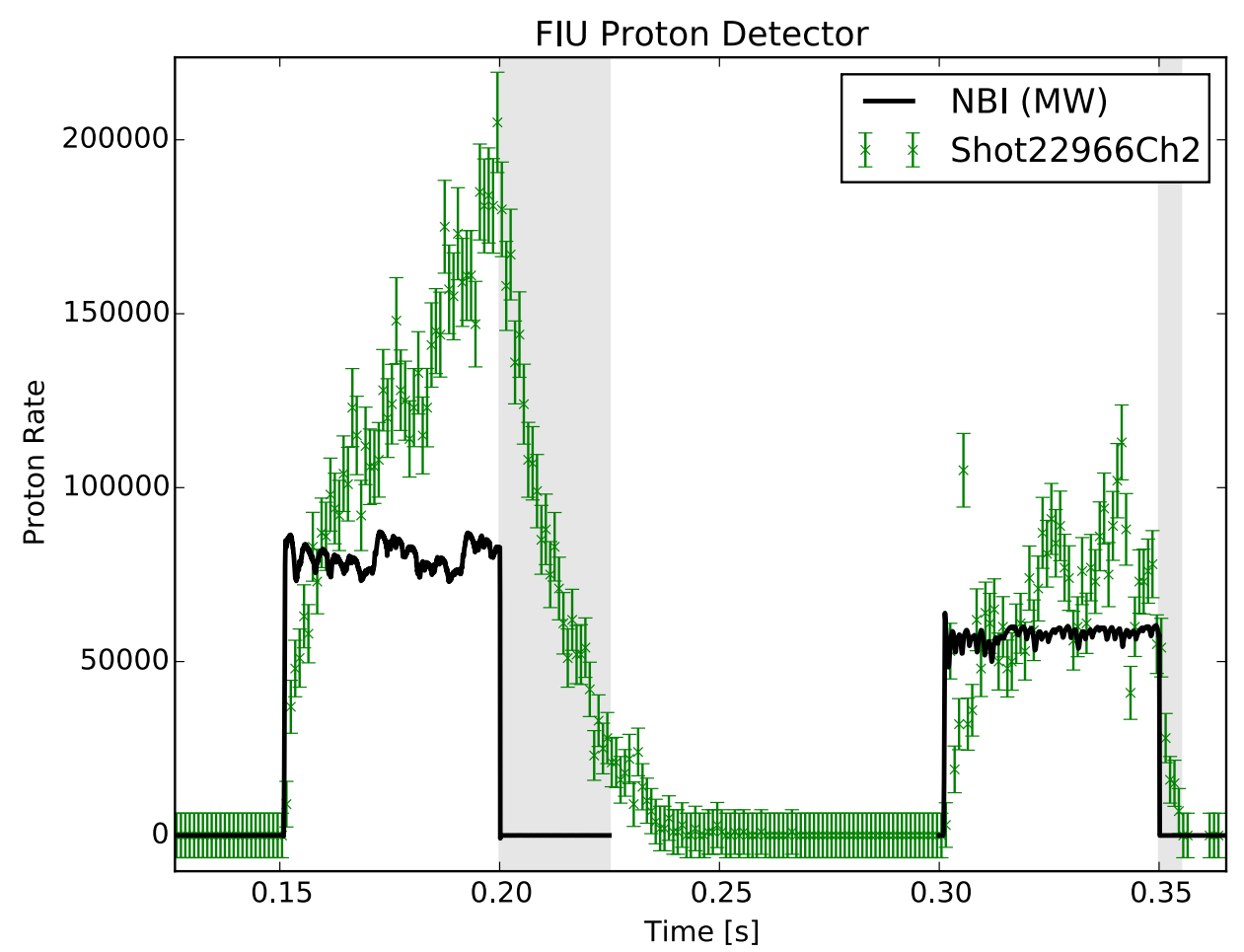

Figure 6.2: During several MAST discharges, two successive neutral beams were injected into the plasma. The regions of exponential decay after NBIs end are highlighted in gray. Note that the first NBI power reaches approximately 1.5 MW and the second NBI power reaches approximately $1 \mathrm{MW}$.

general, the first NBI is turned off at $0.2 \mathrm{~s}$ and the second NBI, depending on the particular shot, is turned off after $0.3 \mathrm{~s}$. Example results of finding the time decay constants for time intervals after the NBI is turned off can be seen in Figure 6.3. Fitting an exponential function to the data from the MAST fission chamber's (FC) global neutron production rates are also displayed in Figure 6.3. The time decay constants for protons and neutrons during several shots are also summarized in Table $6.1^{1}$ and Table 6.2.

\footnotetext{
${ }^{1}$ Note that pulses were not resolved from PD channel 0 data because the channel's detector and electronics were saturated.
} 


\begin{tabular}{|c|c|c|c|c|c|}
\hline Diagnostic & Shot & Channel & $\tau_{1}[\mathrm{~ms}]$ & $\tau_{2}[\mathrm{~ms}]$ & $\mathrm{R}[\mathrm{m}]$ \\
\hline PD Protons & 29958 & 1 & $10.2+/-0.6$ & $8.7+/-2.5$ & $0.86+/-0.03$ \\
\hline PD Protons & 29958 & 2 & $11.4+/-0.7$ & $8.9+/-1.6$ & $0.93+/-0.03$ \\
\hline PD Protons & 29958 & 3 & $12.4+/-0.6$ & $9.5+/-2.6$ & $1.00+/-0.03$ \\
\hline PD Protons & 29960 & 1 & $15.0+/-1.6$ & $9.9+/-5.3$ & $0.78+/-0.03$ \\
\hline PD Protons & 29960 & 2 & $15.1+/-1.4$ & $5.2+/-0.6$ & $0.84+/-0.03$ \\
\hline PD Protons & 29960 & 3 & $14.0+/-0.8$ & $6.6+/-1.1$ & $0.89+/-0.03$ \\
\hline PD Protons & 29966 & 1 & $14.3+/-0.8$ & - & $0.80+/-0.03$ \\
\hline PD Protons & 29966 & 2 & $13.5+/-0.5$ & $12.3+/-3.4$ & $0.86+/-0.03$ \\
\hline PD Protons & 29966 & 3 & $13.0+/-0.7$ & - & $0.91+/-0.03$ \\
\hline PD Protons & 29972 & 1 & $11.0+/-0.8$ & $8.5+/-2.9$ & $0.80+/-0.03$ \\
\hline PD Protons & 29972 & 2 & $10.4+/-0.5$ & $8.8+/-1.5$ & $0.85+/-0.03$ \\
\hline
\end{tabular}

Table 6.1: This table summarizes the time decay constants for protons, $\tau_{1}$ and $\tau_{2}$, during shots in which two subsequent NBIs take place. $\mathrm{R}$ refers to the midplane radius value for each channel. Background noise in the waveform data prevented the calculation of proton rates, and therefore time decay constants, for a few channels.

\begin{tabular}{|c|c|c|c|}
\hline Diagnostic & Shot & $\tau_{1}[\mathrm{~ms}]$ & $\tau_{2}[\mathrm{~ms}]$ \\
\hline FC Neutrons & 29958 & $9.2+/-0.02$ & $6.3+/-0.03$ \\
\hline FC Neutrons & 29960 & $11.8+/-0.02$ & $7.5+/-0.02$ \\
\hline FC Neutrons & 29966 & $11.7+/-0.02$ & $2.4+/-0.01$ \\
\hline FC Neutrons & 29972 & $9.1+/-0.02$ & $3.2+/-0.06$ \\
\hline
\end{tabular}

Table 6.2: This table summarizes the time decay constants for neutrons, $\tau_{1}$ and $\tau_{2}$, during shots in which two subsequent NBIs take place. The fission chamber instrument is represented by FC. 

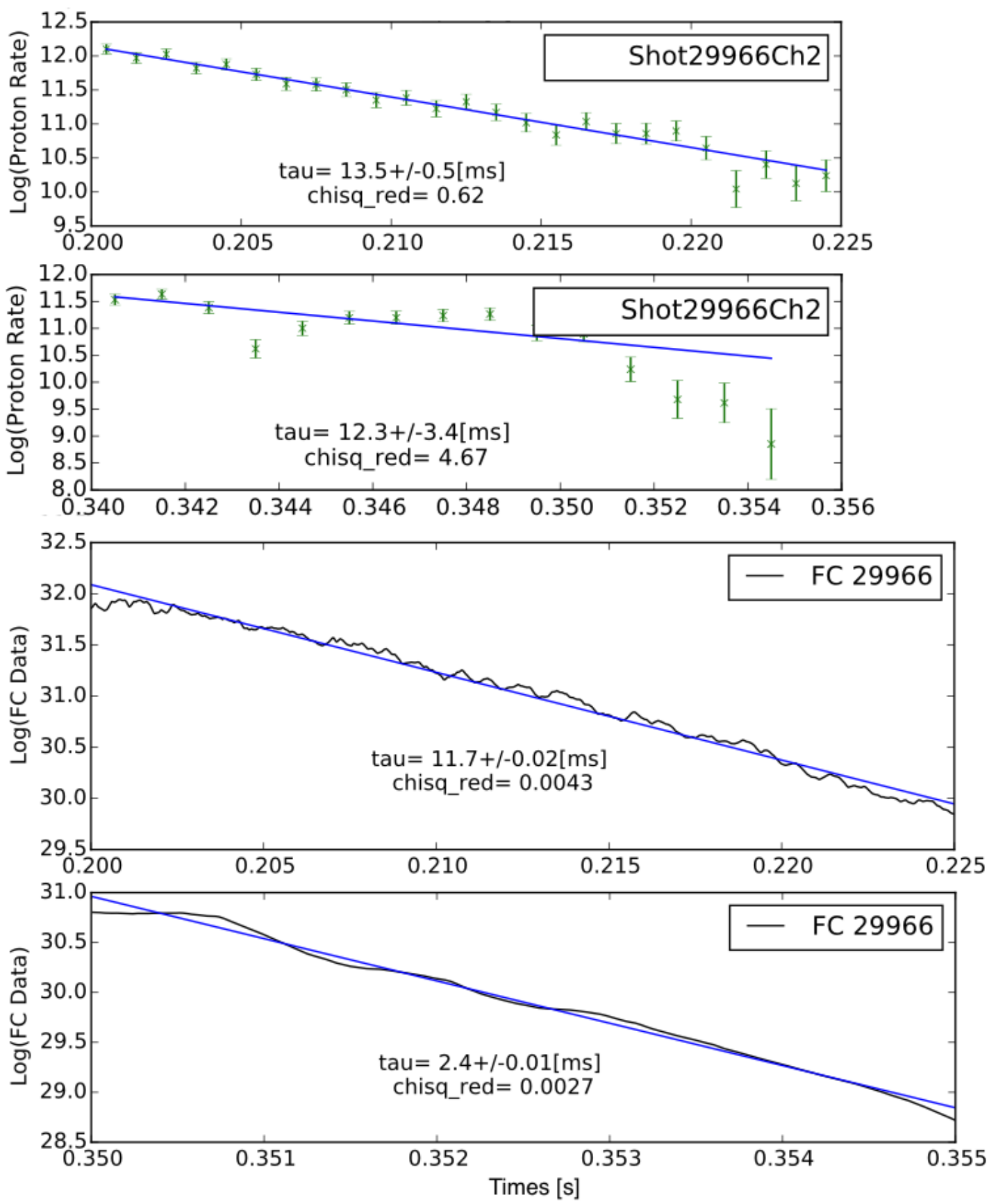

Figure 6.3: Above are the experimental proton and neutron time decay constants, $\tau$ (tau), for two different time intervals. Data from the fission chamber (FC) are used for the neutron time decay constants. 
Figure 6.4 compares the proton and neutron decay time constants to each other. The PD channel 2 and channel 3 values for $\tau_{2}$ from pulse 29960, diverge from the other $\tau$ values measured by the PD and FC. ${ }^{1}$ Therefore, a fit (Fit1 in Figure 6.4) without these two data points was used to find a correlation between the time decay constants; Fit2 fits all data points. The range of experimental values of $\tau$ is not large enough to adequately compare to results from Tournianski et al. (2005), which had experimental $\tau_{R}$ values up to $80 \mathrm{~ms}$.[69]

Figure 6.5 displays $\tau_{1}$ and $\tau_{2}$ as a function of mid-plane radii. For shots 29960, 29966, and 29972, $\tau_{1}$ decreases towards the corresponding neutron $\tau_{1}$ along a similar trend (though their initial values range from $10 \mathrm{~ms}$ to $15 \mathrm{~ms}$ ). Conversely, $\tau_{2}$ for shots 29960 and 29972 increase towards the corresponding neutron $\tau_{1}$. Both $\tau_{1}$ and $\tau_{2}$ for shot 29958 increase with larger mid-plane radii. Though the comparison between the PD and FC data is promising, more experiments with an exponential decay of proton and neutron rates are needed to indicate how well the PD might substitute for or complement a neutron measurement diagnostic. Future work in combining these proton time decay values with data from the MAST neutron camera, which typically samples the plasma at larger mid-plane radial values, could also provide a more clear relation between the slowing down time and radial distance in the plasma.

\footnotetext{
${ }^{1}$ The reason as to why this divergence occurred has not yet been determined.
} 


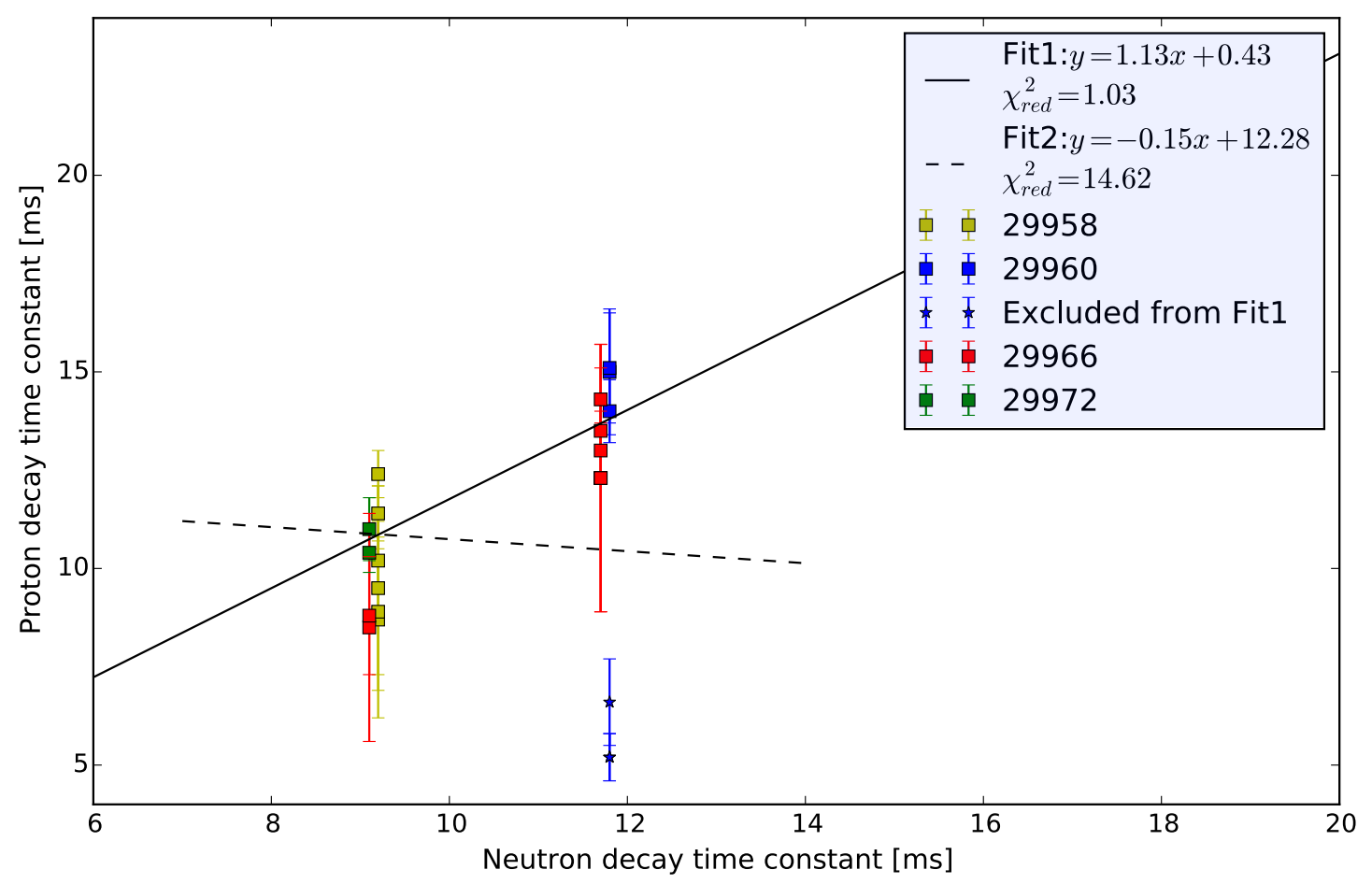

Figure 6.4: The experimental proton time decay constants as a function of experimental neutron time decay constants. The legend for the plot is in a box which is shaded light blue. 

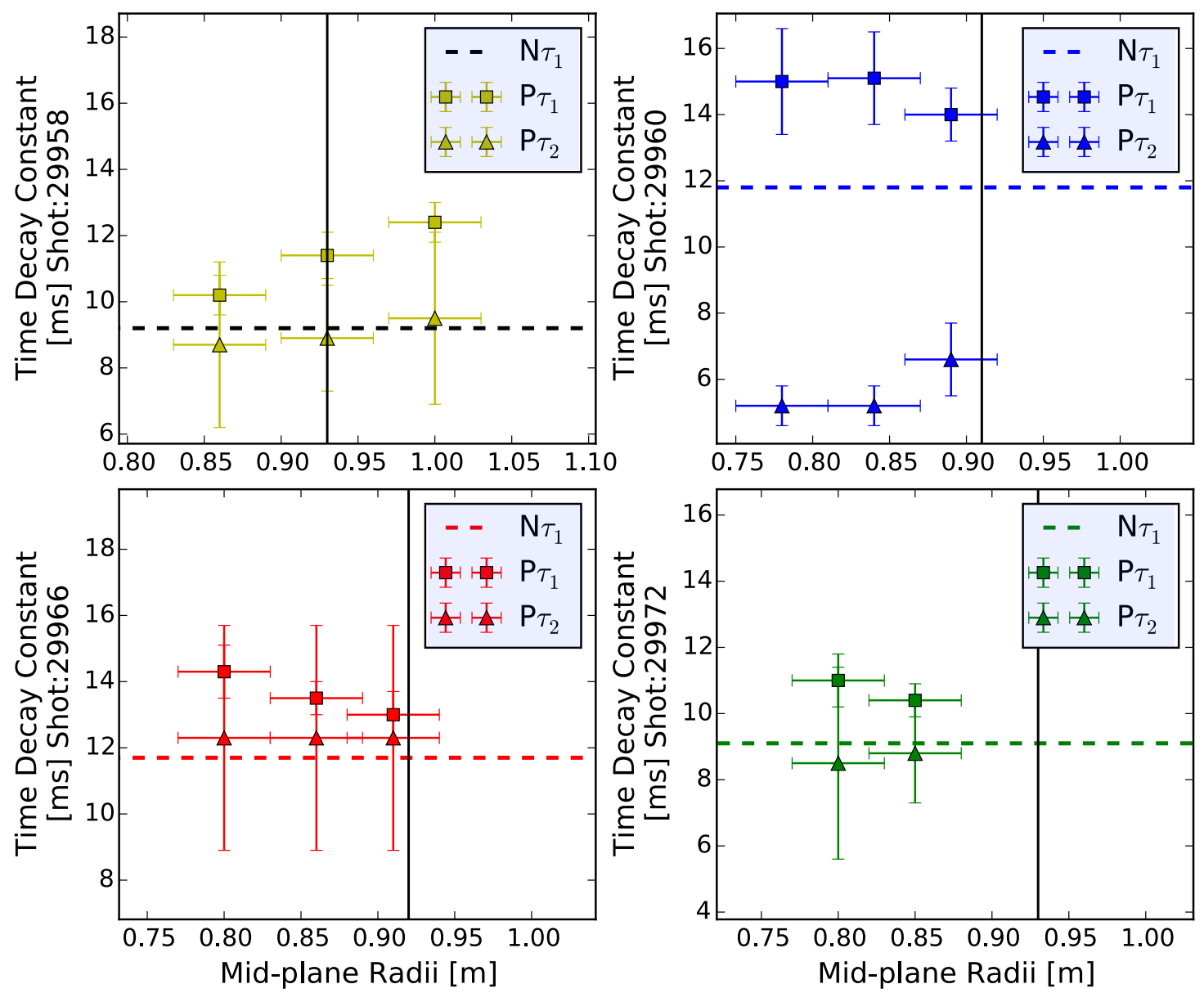

Figure 6.5: The experimental proton and neutron time decay constants, $\tau_{1}$ and $\tau_{2}$ as a function of mid-plane radii. the radial value for the magnetic axis is plotted as the solid black vertical line in each figure. The legend for each plot is in a box which is shaded light blue. 


\subsection{Proton Production Rates}

The measurement of proton production rates with a resolution of $1 \mathrm{~ms}$ enables the observation of oscillation effects on the fast ion population through observing protons during the NBI heating of the plasma.

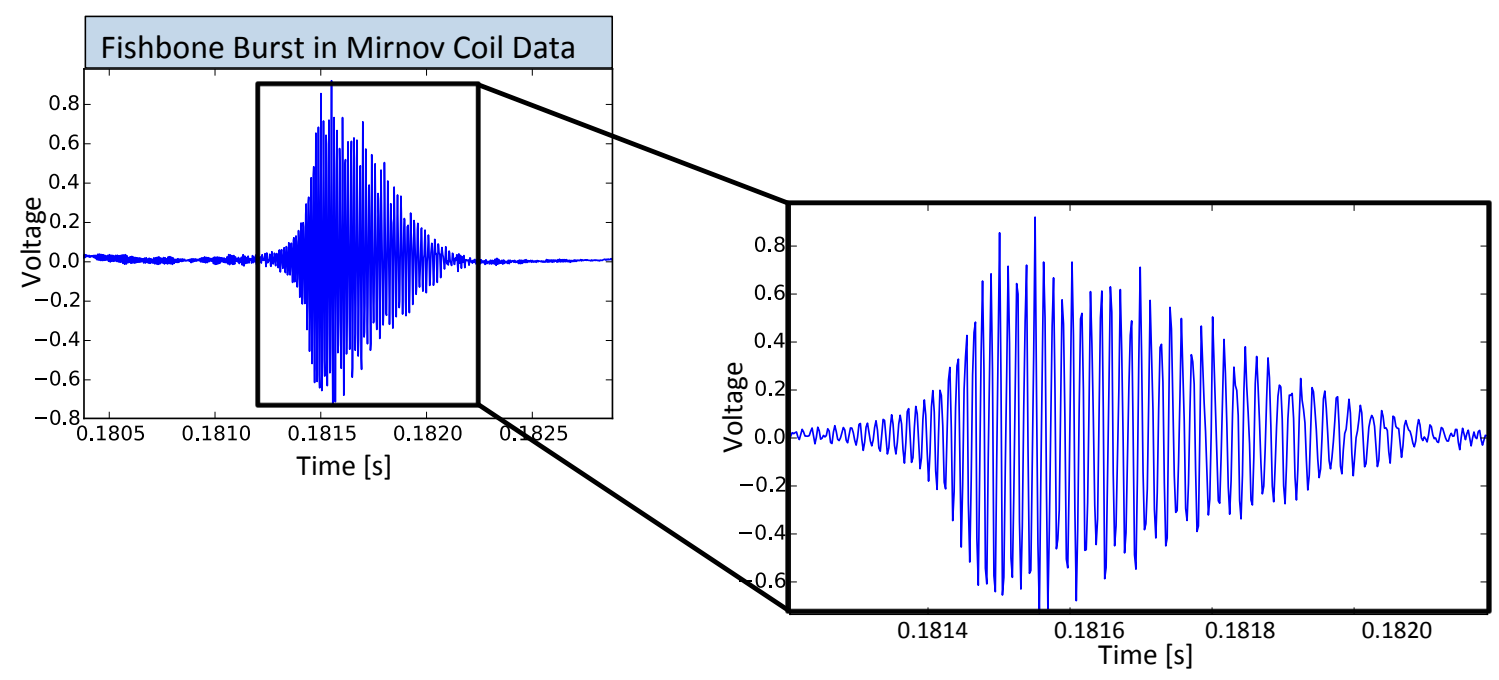

Figure 6.6: Above is a qualitative image displaying an example fishbone event observed in Mirnov coils.

Fishbones As discussed in Chapter 1 the magnetic field lines within the plasma lie on toroidal flux surfaces, which are also surfaces of constant poloidal flux. Each field line is characterized by a parameter $\mathrm{q}$. The numerical value of $\mathrm{q}$ is the number of times a field line travels around the plasma toroidally in order to complete one full revolution poloidally. The field lines do not necessarily close on themselves, except when q is a rational number. For example when $\mathrm{q}$ is a rational number, a field line can travel $m$ times around the plasma in the toroidal direction and $n$ times around the plasma in the poloidal direction before it closes on itself. The ratio of the number of toroidal and poloidal rotations, also called mode 
numbers, in the plasma describes $q$ in (6.42).

$$
q=\frac{m}{n}
$$

Because field lines travel along a constant flux surface, q values are used to describe the flux surfaces as well. Field lines which travel one time around the plasma in the toroidal direction $(m=1)$ and one time around the plasma in the poloidal direction $(n=1)$, before closing on themselves, travel along a $\mathrm{q}=1$ surface.

The collective effect of energetic ions moving in the plasma are a means of energy transfer within the plasma. When fast ions resonate with oscillations on a surface of the plasma, instabilities can occur. Fishbone (FB) bursts can occur on $q=1$ surfaces when fast ions travel once around the plasma in both the toroidal and poloidal direction during an orbital period.

Additionally, changes in pressure can trigger oscillations. A value called $\beta$ was introduced in Chapter 1 as the ratio of the plasma pressure to the magnetic energy density.

$$
\beta=p\left(\frac{2 \mu_{0}}{B^{2}}\right)
$$

When B is evaluated as the poloidal magnetic field $B_{\theta}$ and the pressure is evaluated as the pressure created by trapped energetic particles which have a thermal kinetic energy greater than the background plasma, then the ratio can be referred to as $\beta_{p}$. [58] Changes in the energetic ion pressure in the plasma core can change the value of $\beta_{p}$.[70]

Gradients in the fast ion density and the temporary loss of toroidal symmetry in the magnetic equilibrium can also drive plasma oscillations, such as FBs, which cause the radial transport of fast ions within the plasma. Many FB events, or bursts, can occur throughout a shot where fast ions are intermittently ejected from the plasma (a series of fast ion 
ejections). ${ }^{1}$ Fishbones can even trigger sawtooth events, thereby further reducing the fast ion confinement.[70] Though not destructive enough to dissipate the plasma, these FBs occur often enough in MAST discharges to reduce the fast ion confinement, and their effect can be observed through changes in fusion reaction rates.[70]

The basic description of FBs and their relation to fast ion confinement above, provides the motivation for developing diagnostics to measure their effects on a plasma. In addition, there are numerous other oscillations and instabilities that can have an adverse effect on fast ion confinement. As stated before, relationships between instabilities to fast ion confinement is complex and not completely understood in the plasma physics and fusion research communities. The complexity of these relations highlight the importance of empirical methods to observe the effects of oscillations and instabilities, which can be achieved through developing a diagnostic such as the PD to study changes in the fusion reaction rates. In general, improved diagnostics studying fast ion populations have enabled formulation of improved theoretical and numerical models of oscillation and instability effects and it is hoped that development of the PD will further contribute to this progress. While FBs are seen in Mirnov coil oscillations ${ }^{2}$, their effects can also be seen through charged fusion product diagnostics. One of the goals of this dissertation work and the development of the PD is to experimentally observe the effect of oscillations, such as FBs, which can lead to changes in the density profile of neutral beam ions and the production of protons emitted from DD fusion reactions.

Events Plasma pulse, or shot, 29909 had approximately 1.6 MW of NBI power and 800 $\mathrm{kA}$ of toroidal plasma current. The core electron temperature was $0.7 \mathrm{keV}$, core electron density was approximately $0.3 \times 10^{14} \mathrm{~m}^{-3}$, and total neutron production rates were on the

\footnotetext{
${ }^{1}$ The radial transport of fast ions in a plasma with FBs is higher than that of a plasma without oscillations and MHD instabilities (quiescent plasma).

${ }^{2}$ Mirnov coils measure oscillations in the magnetic field.
} 
order of $0.39 \times 10^{14} \mathrm{~Hz}$. Additionally, these low electron density discharges created a $25 \mathrm{~ms}$ time interval (from $235 \mathrm{~ms}$ to $260 \mathrm{~ms}$ ) of low MHD activity.[13][12] Figure 6.7 displays all PD data channels, Fission Chamber data (FC), Mirnov coil magnetic field oscillations data, and NBI power.
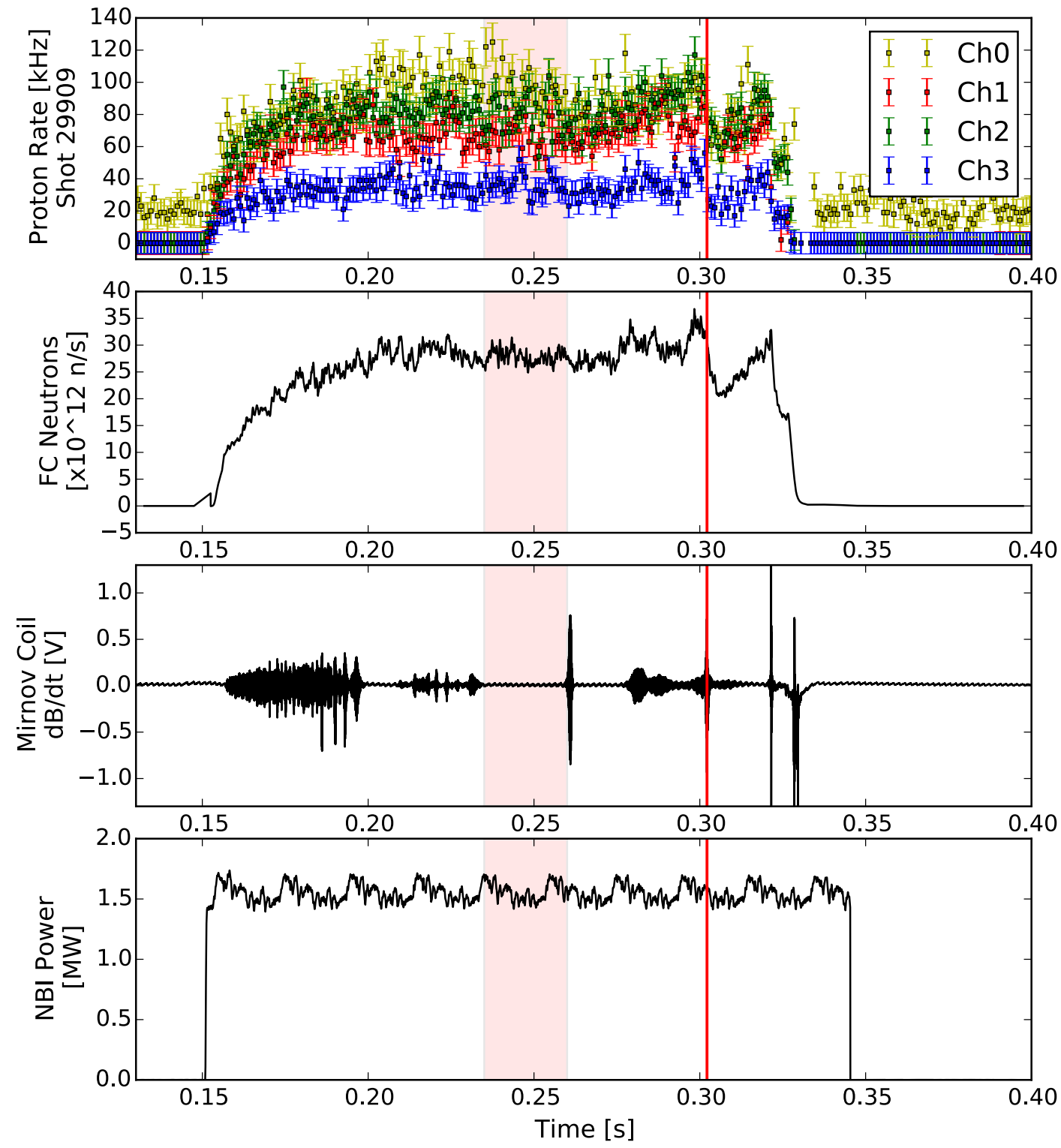

Figure 6.7: Above are data for plasma pulse 29909 from the Proton Detector (all channels), Fission Chamber, Mirnov Coil, and NBI power. The red vertical line indicates an event of interest to be discussed. The quiescent period, 235 $\mathrm{ms}$ to $260 \mathrm{~ms}$, is shaded in red. 
Because no fast variation in events occurs during the quiescent period from $235 \mathrm{~ms}$ to $260 \mathrm{~ms}$, the integration time for the proton rates in this period is increased from $1 \mathrm{~ms}$ to $5 \mathrm{~ms}$ to average out statistical fluctuations. The time period is shaded in red in Figure 6.8. After the period of low activity, an FB event is observed in the Mirnov Coil data at approximately $300 \mathrm{~ms}$. The event can more clearly be seen in Figure 6.10. Note that as a reference for low activity during this plasma pulse, PD data from a time interval during the quiescent period is included in the plots (dashed magenta line). The FB is accompanied by an approximate $35 \%$ drop in the total neutron rate and up to a $65 \%$ drop in the proton rate. Note that the proton rate is in relation to local regions of the plasma while the FC constructs the global neutron rate emitted from the plasma. The differences between changes in the proton rate are observed within individual channels. For example, channel 0 and channel 2 experience a $40 \%$ and $50 \%$ reduction, respectively, in the proton rate while channel 3 has a reduction by up to $65 \%$. When comparing the mid-plane radii for PD channels the largest drop in proton rates is seen in the channel with a mid-plane radii closest to the plasma core (see channel 3 in blue in Figure 6.9). As the mid-plane radii for each channel move farther from the plasma core, the drop in the experimental proton rates gradually decreases in this order: channel 3, channel 2, channel 0 , and channel 1 . This corresponds with the simulated proton emission from the orbit code.

These fluctuations in proton production rates reflect fluctuations in the DD fusion reaction rates in the plasma. Drops in the DD fusion reaction rates coincide with the FB event for the PD and FC. 


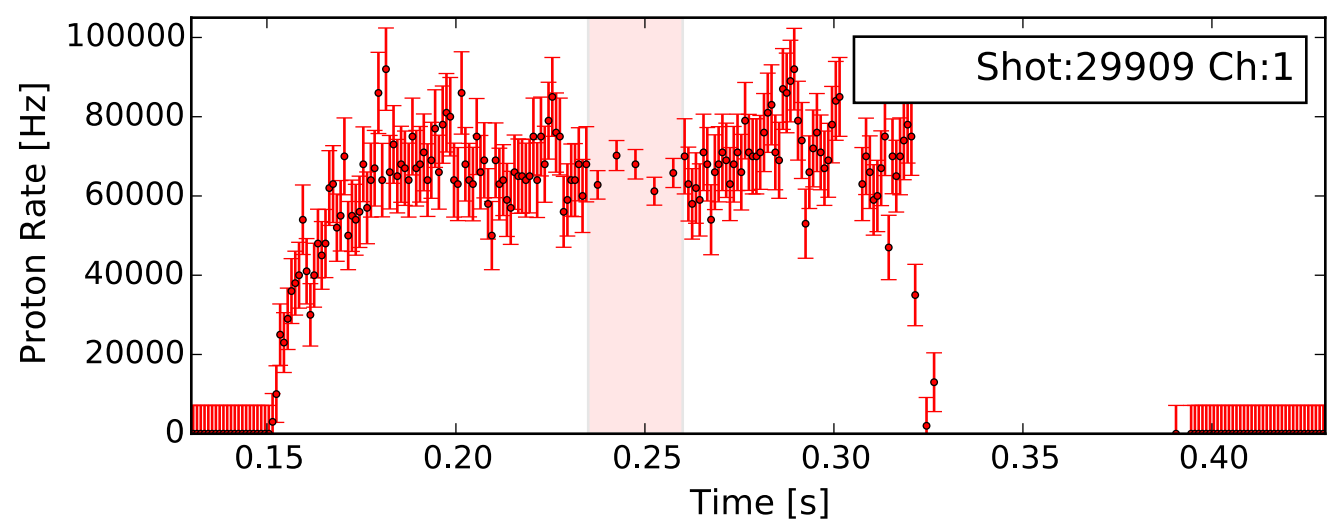

Figure 6.8: Above are PD channel 1 rates with the quiescent period, $235 \mathrm{~ms}$ to $260 \mathrm{~ms}$, is shaded in red.

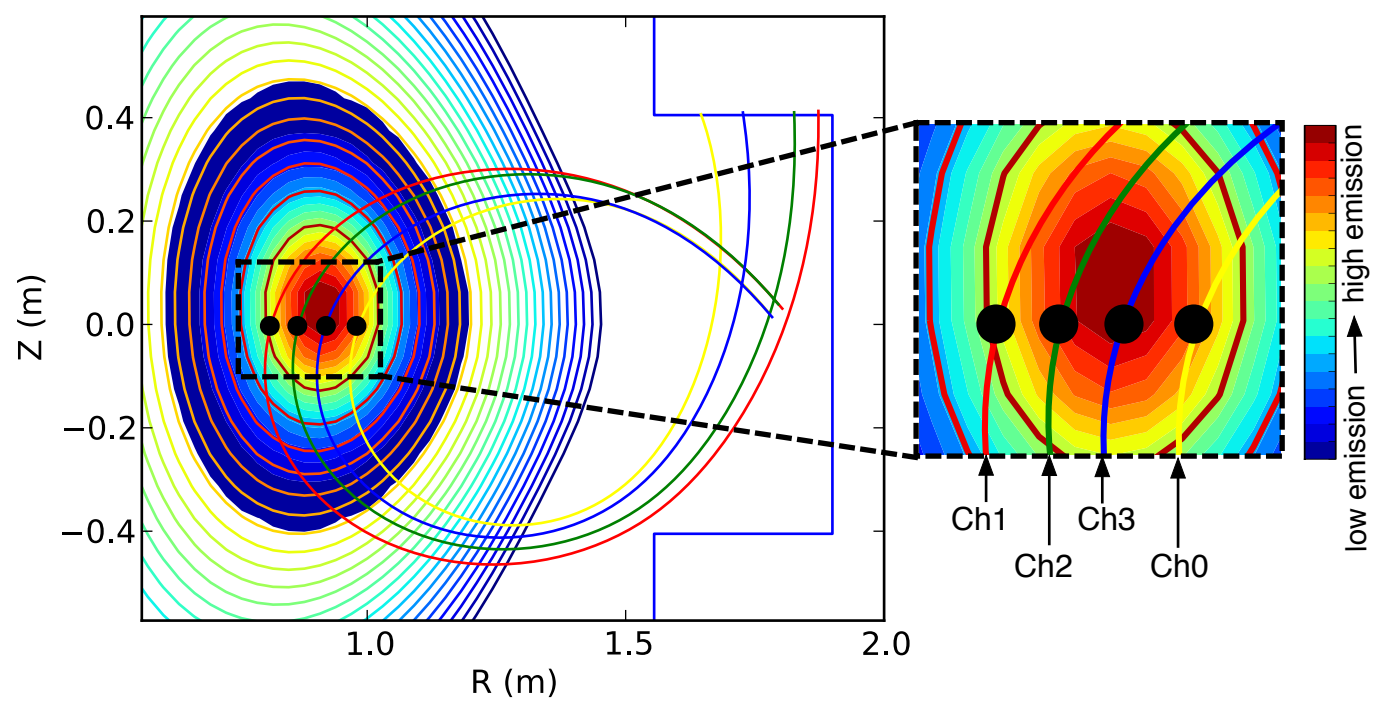

Figure 6.9: Above is the orbit output for the poloidal trajectories for each PD channel. The mid-plane radii for each channel is labeled above.The mid-plane radii for channels $0,1,2$, and 3 are approximately $98.5+/-3 \mathrm{~cm}, 81.5+/-3 \mathrm{~cm}$, $86.8+/-3 \mathrm{~cm}$, and $92+/-3 \mathrm{~cm}$, respectively. 

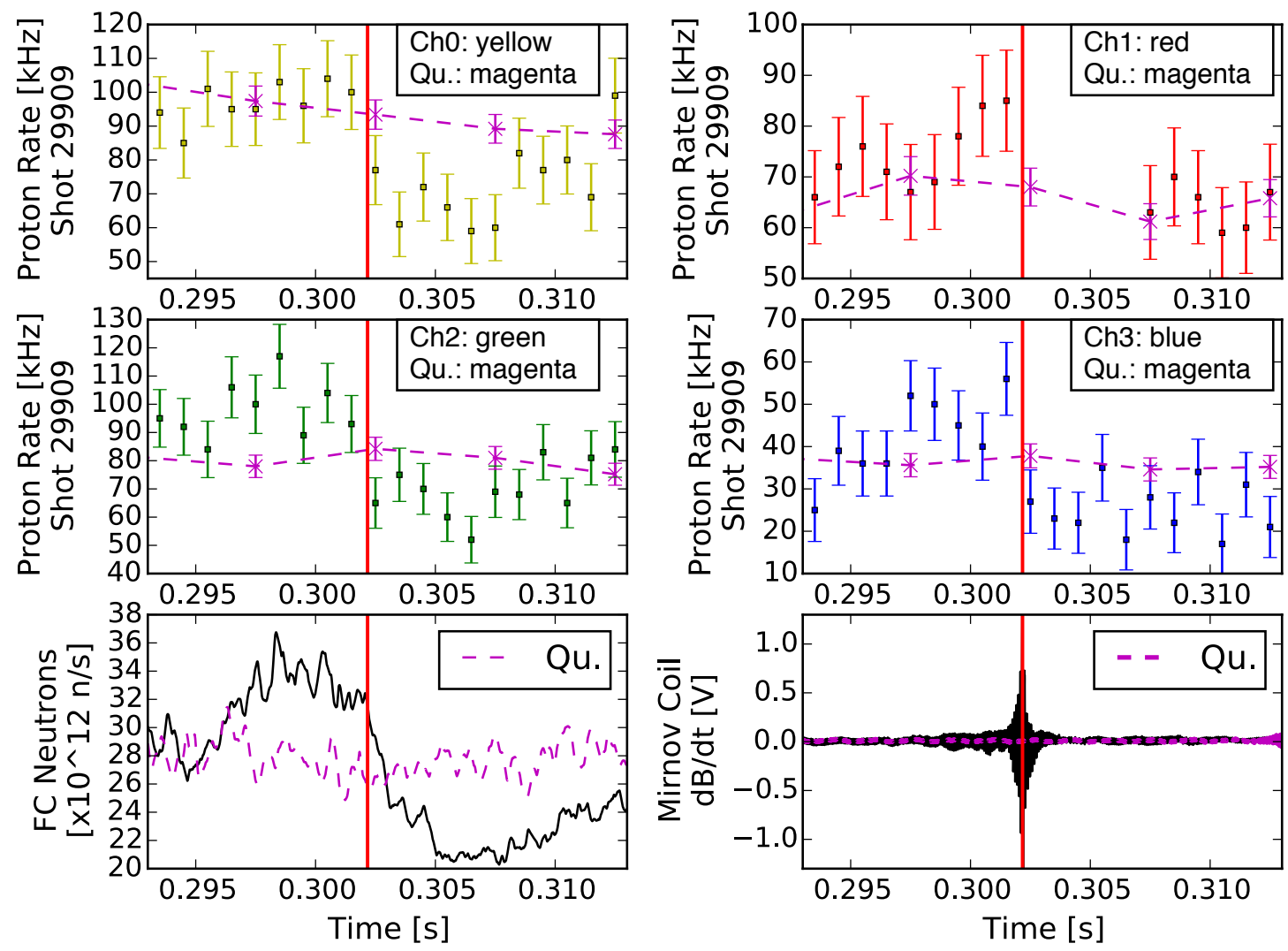

Figure 6.10: Above are data for plasma pulse 29909 from the Proton Detector, Fission Chamber, and Mirnov Coil. Rates from a time interval during the quiescent period are labeled as $Q u$. There is a gap in channel 1 rates (around 305 ms) because pulses were not able to be resolved from noise signals in the raw data during that specific time interval. The red vertical line indicates an event of interest to be discussed. 
A higher density plasma, core electron density was approximately $3.5 \times 10^{19} \mathrm{~m}^{-3}$, than pulse 29909 was created with pulse 29931. The NBI power and toroidal plasma current were similar to 29909. This pulse, however, had a higher core electron temperature of $1.3 \mathrm{keV}$. Total neutron production rates were on the order of $0.35 \times 10^{14} \mathrm{~Hz} .[13][12]$ The PD Data channels, Fission Chamber data, Mirnov coil magnetic field oscillations data, and NBI power are displayed in Figure 6.11. At approximately $268 \mathrm{~ms}$ an FB event occurs in the Mirnov Coil data, see Figure 6.12. The proton production rates fall approximately 24\%, $30 \%$, and $55 \%$ for Channels 1,2, and 3, respectively. The drop in the rates compares with a $30 \%$ drop in the global neutron rate.

The poloidal trajectories for the PD channels, in Figure 6.13, reveal why there are no rates data for channel 0 (yellow trajectory). Instead of sampling a region of the plasma in which protons leave the plasma before completing one orbit, the channel 0 poloidal trajectory samples two regions near the plasma core. The channel's detector and electronics were saturated with too many incident particles; therefore the pulses were not able to be resolved from the raw data. The mid-plane radii for channels 1, 2, and 3 are approximately $82.1+/-3 \mathrm{~cm}, 87.4+/-3 \mathrm{~cm}$, and $92.5+/-3 \mathrm{~cm}$, respectively. The first mid-plane radii for channel 0 is approximately $100.4+/-3 \mathrm{~cm}$.

Similar to the last FB event, when comparing the mid-plane radii for PD channels the largest drop in proton rates are seen in the channel with a mid-plane radii closest to the plasma core (see channel 3 in blue in Figure 6.13). As the mid-plane radii for each channel move farther from the plasma core, the drop in the experimental proton rates gradually decreases in this order: channel 3, channel 2, and channel 1. This also corresponds with the simulated proton emission from the orbit code. Plotting the falls in DD proton and global neutron rates as a function of mid-plane radii, in Figure 6.14, indicates that the largest decreases in fast ion rates occur closest to the plasma core. 

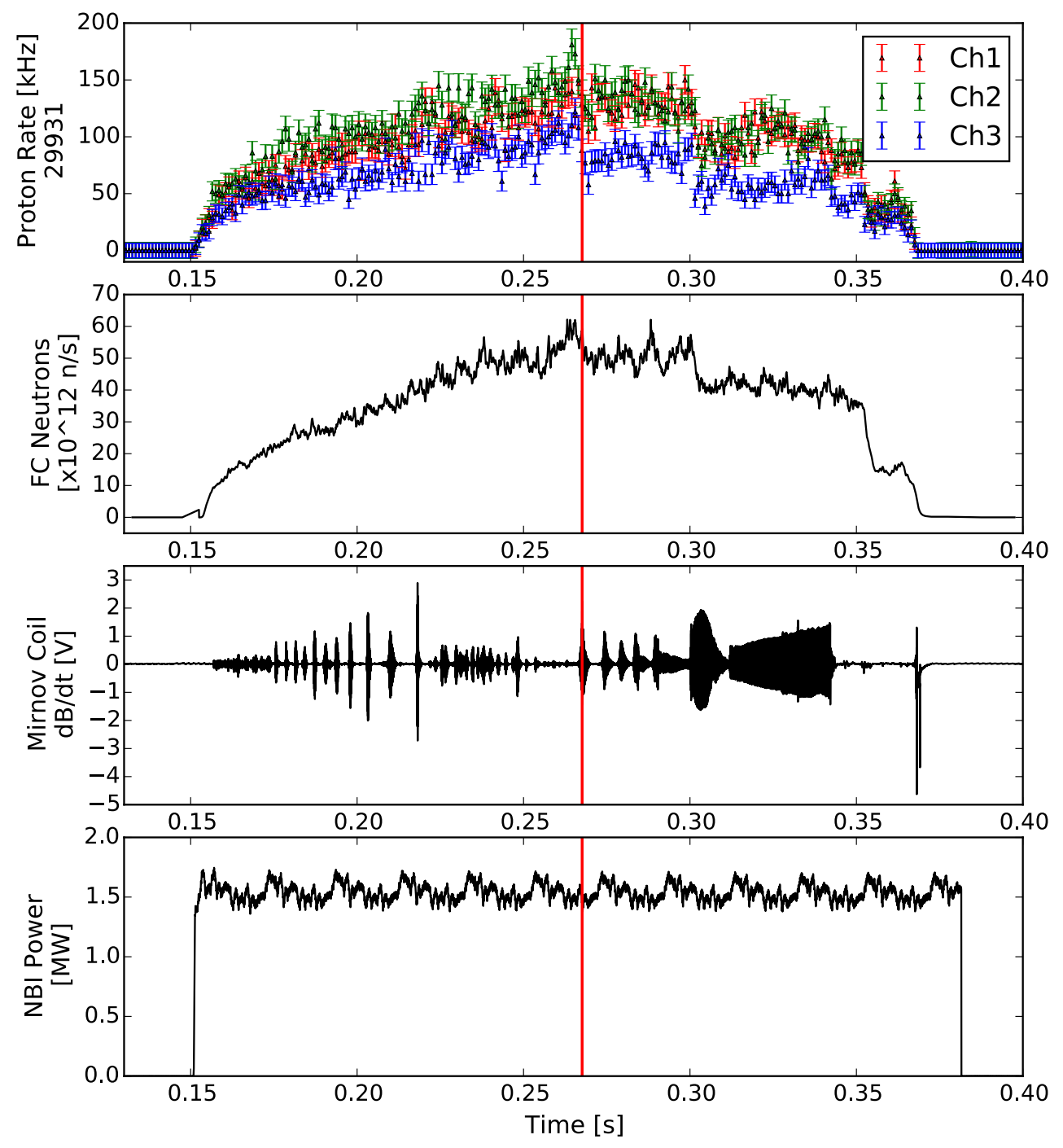

Figure 6.11: Above are data for plasma pulse 29931 from the Proton Detector, Fission Chamber, Mirnov Coil, and NBI power. The red vertical line indicates an event of interest to be discussed. 

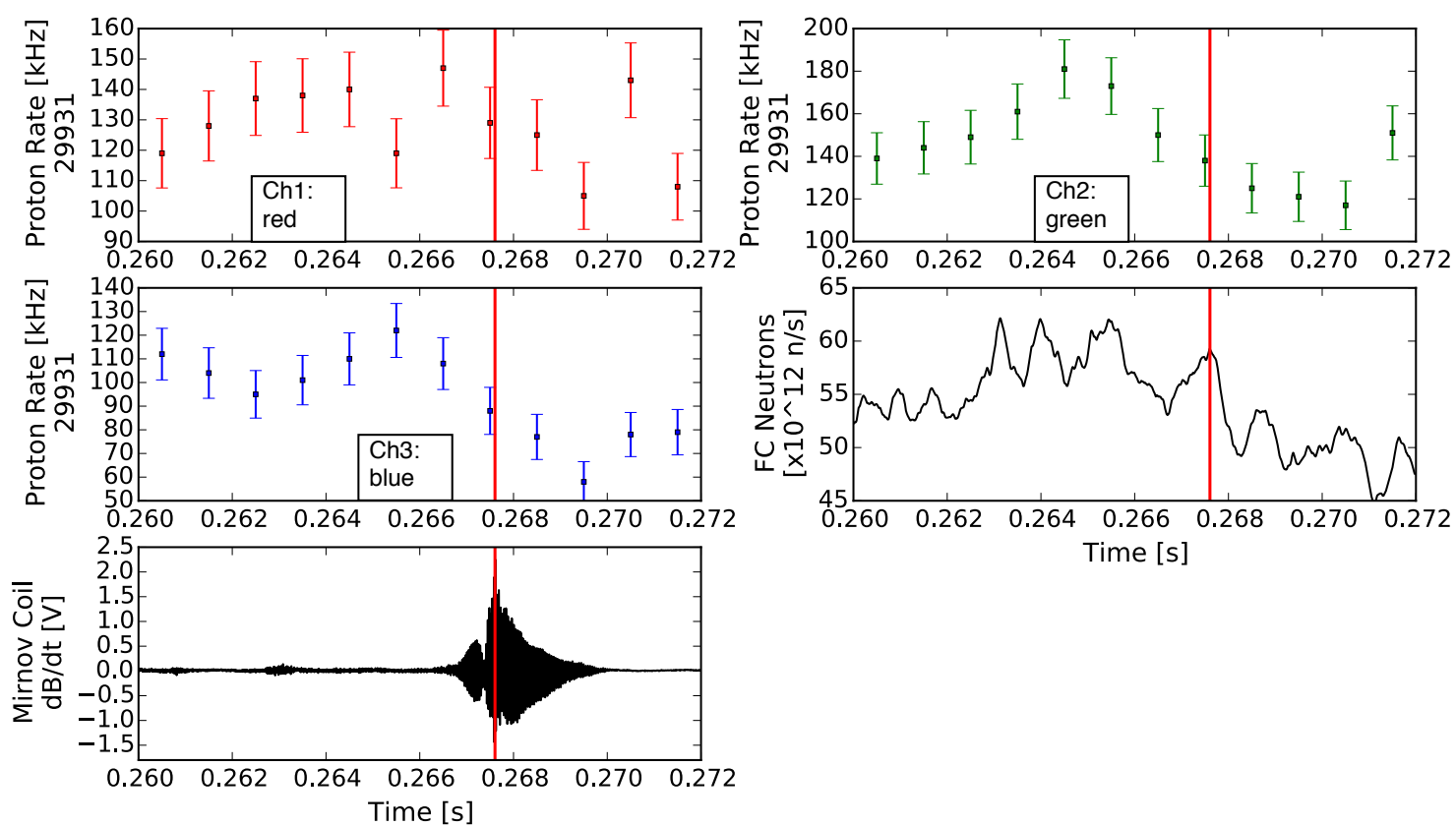

Figure 6.12: Above are data for plasma pulse 29931 from the Proton Detector, Fission Chamber, Mirnov Coil, and NBI power. The red vertical line indicates an event of interest to be discussed.

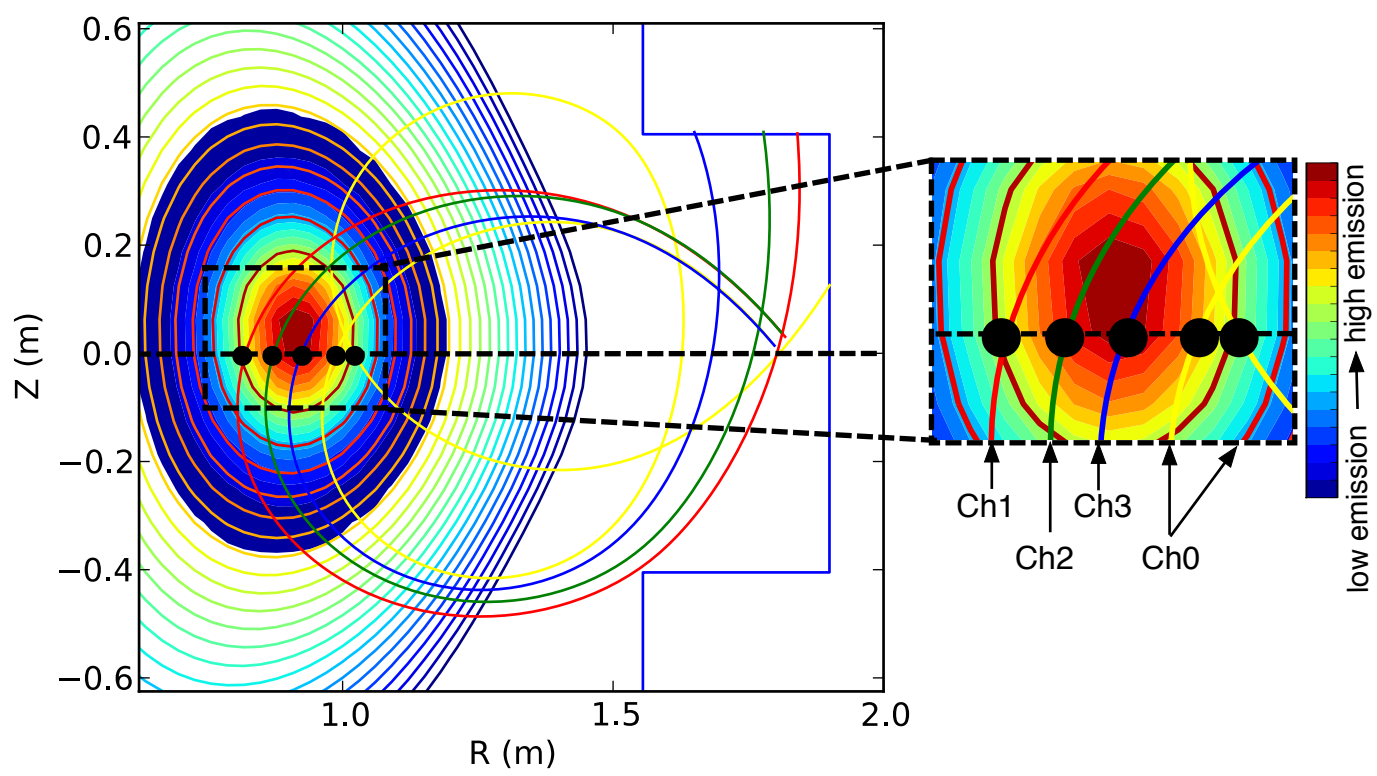

Figure 6.13: Above is the orbit output for the poloidal trajectories for each PD channel. The mid-plane radii for each channel is labeled above.The midplane radii for channels 1,2 , and 3 are approximately $82.1+/-3 \mathrm{~cm}, 87.4+/-3$ $\mathrm{cm}$, and $92.5+/-3 \mathrm{~cm}$, respectively. The first mid-plane radii for channel 0 is approximately $100.4+/-3 \mathrm{~cm}$. 

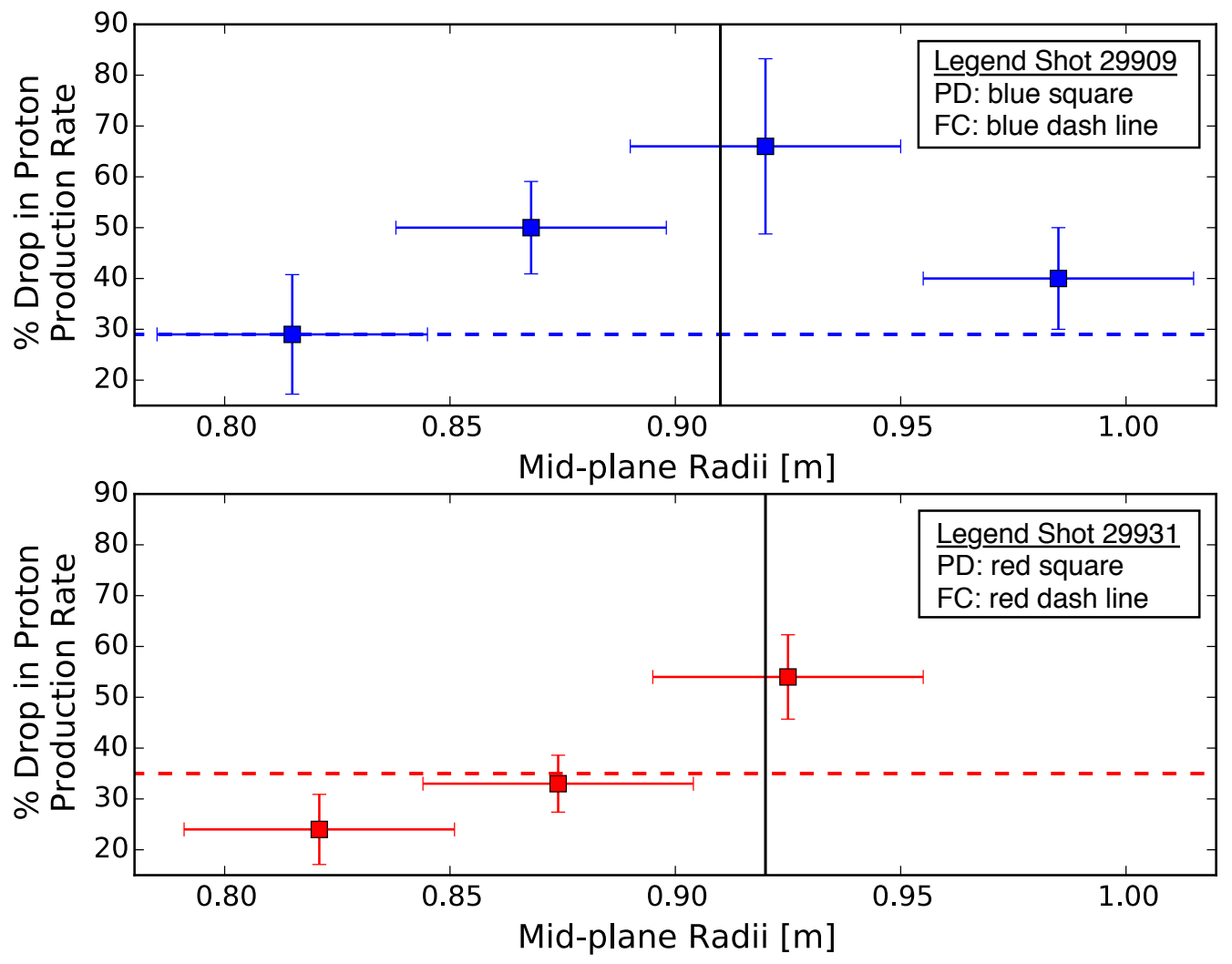

Figure 6.14: Plotting the falls in DD proton and global neutron rates as a function of mid-plane radii indicates that the largest decrease in fast ion rates occur closest to the plasma core. The black vertical line indicates the radial value of the magnetic axis. For shot 29909, each PD data point from left to right represents: Ch0, Ch1, Ch2, and Ch3. For shot 29931, each PD data point from left to right represents: $\mathrm{Ch} 1, \mathrm{Ch} 2$, and $\mathrm{Ch} 3$. 


\subsection{Proton Emission Profile}

The particle emission from the plasma, or emissivity as introduced in Chapter 2, can be represented as a function of several parameters such as the plasma's poloidal position within the plasma or poloidal magnetic flux. The orbit code uses a function for the emissivity, S, to calculate the simulated particle emission. Using an S of only a few parameters implies a constant particle emission along the nested surfaces of constant flux as well as toroidal symmetry in the plasma.[54] The orbit code calculates S for each segment of a channel's orbit, or central poloidal trajectory. Experimental particle rates can then be fit to determine an appropriate function for $\mathrm{S}$ which can describe the proton emission from a local region of the plasma.

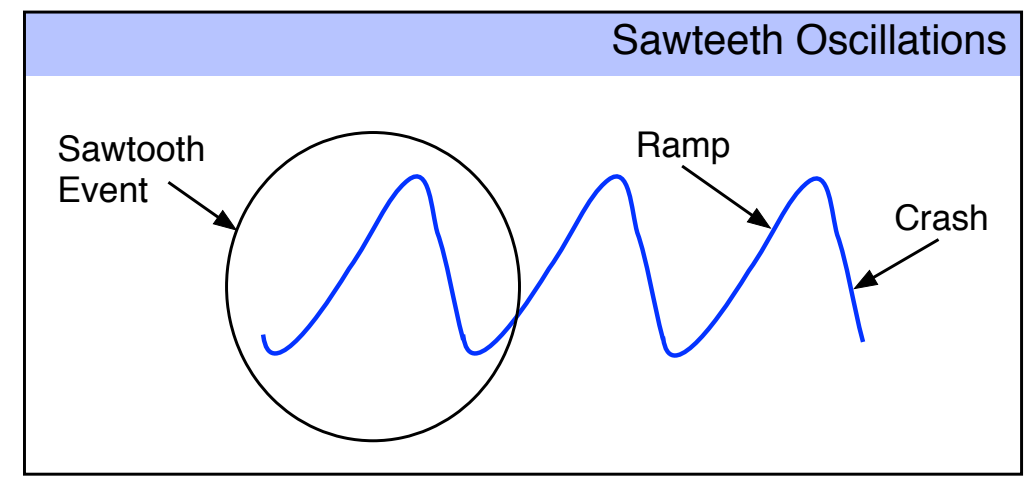

Figure 6.15: Above is a qualitative image displaying sample sawteeth events. The ramp up phase and crash, or collapse, phase are labeled.

Sawteeth Sawtooth oscillations also occur on $q=1$ surfaces. They occur when the current density and temperature in the plasma core increase in a ramping up phase and then decrease in a crash phase (see Figure 6.15 and Figure 6.16). As a consequence ${ }^{1}$, this can lead to an an increase of energy transport from the plasma core to the plasma edge thereby resulting in a reduction of the plasma's fast ion confinement.[58][12] These large fluctuations inside of the plasma core can be observed through changes in fusion reaction rates.

\footnotetext{
${ }^{1}$ During the crash phase, magnetic field lines can tear and reconnect.
} 


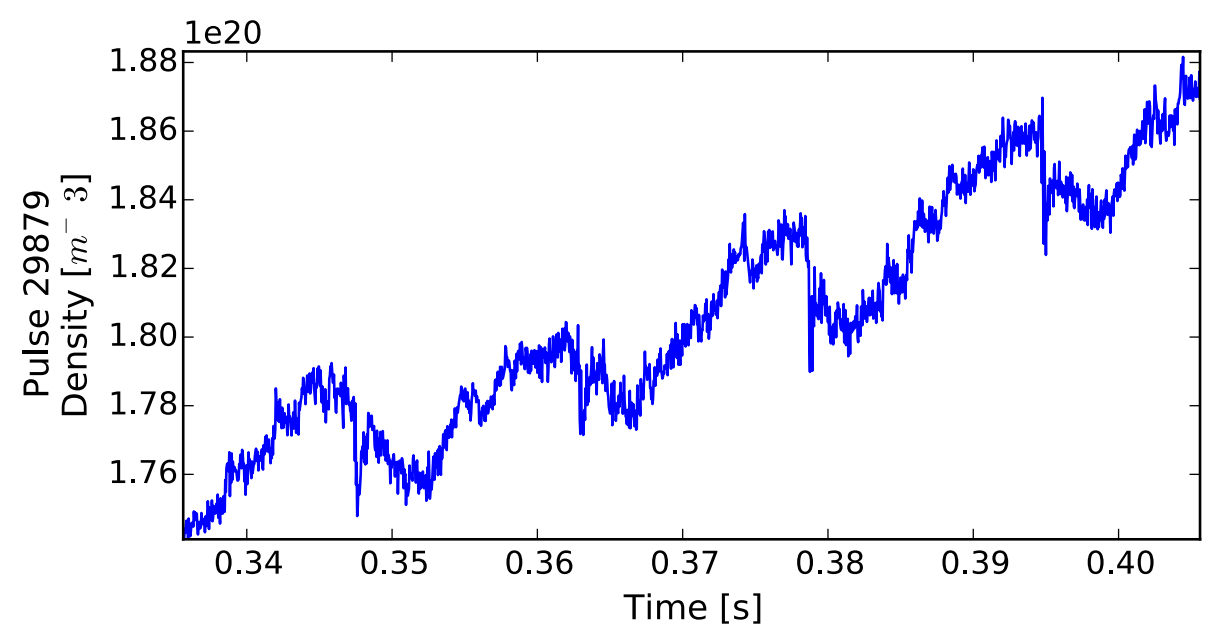

Figure 6.16: Above is a time interval of the plasma electron density during plasma pulse 29879 in which successive sawtooth events can be observed.

Sawtooth crashes are associated with bursts of neutral beam ion loss and rapid drops in neutron and proton emission rates. Though in general they are not disruptive to the plasma, these sawteeth occur often in MAST discharges and can trigger other instabilities.[58]

The basic description of sawteeth and their relation to fast ion confinement above, provides the motivation for developing diagnostics to measure their effects on a plasma. One of the goals of my dissertation work and the development of the PD is to experimentally observe the effect of oscillations, such as sawteeth, on the production of protons emitted from DD fusion reactions.

Event Pulse 29879 had a 0.585 T toroidal magnetic field and 1.5 MW of NBI power. The pulse was also characterized by a $600 \mathrm{kA}$ toroidal plasma current, $1 \mathrm{keV}$ core electron temperature, and neutron rates on the order of $0.4 \times 10^{14} \mathrm{~Hz}$. The neutral beam atoms had an energy of $50 \mathrm{keV}$.[12] Figure 6.17 displays PD and fission chamber (FC) data for this plasma pulse. Figure 6.18 displays PD and neutron camera (NC) data for this plasma pulse. The NC is a collimated neutron detector (CND) system, discussed in Chapter 1, which detects 2.5 MeV neutrons emitted from DD fusion reactions. Therefore, the PD and NC 

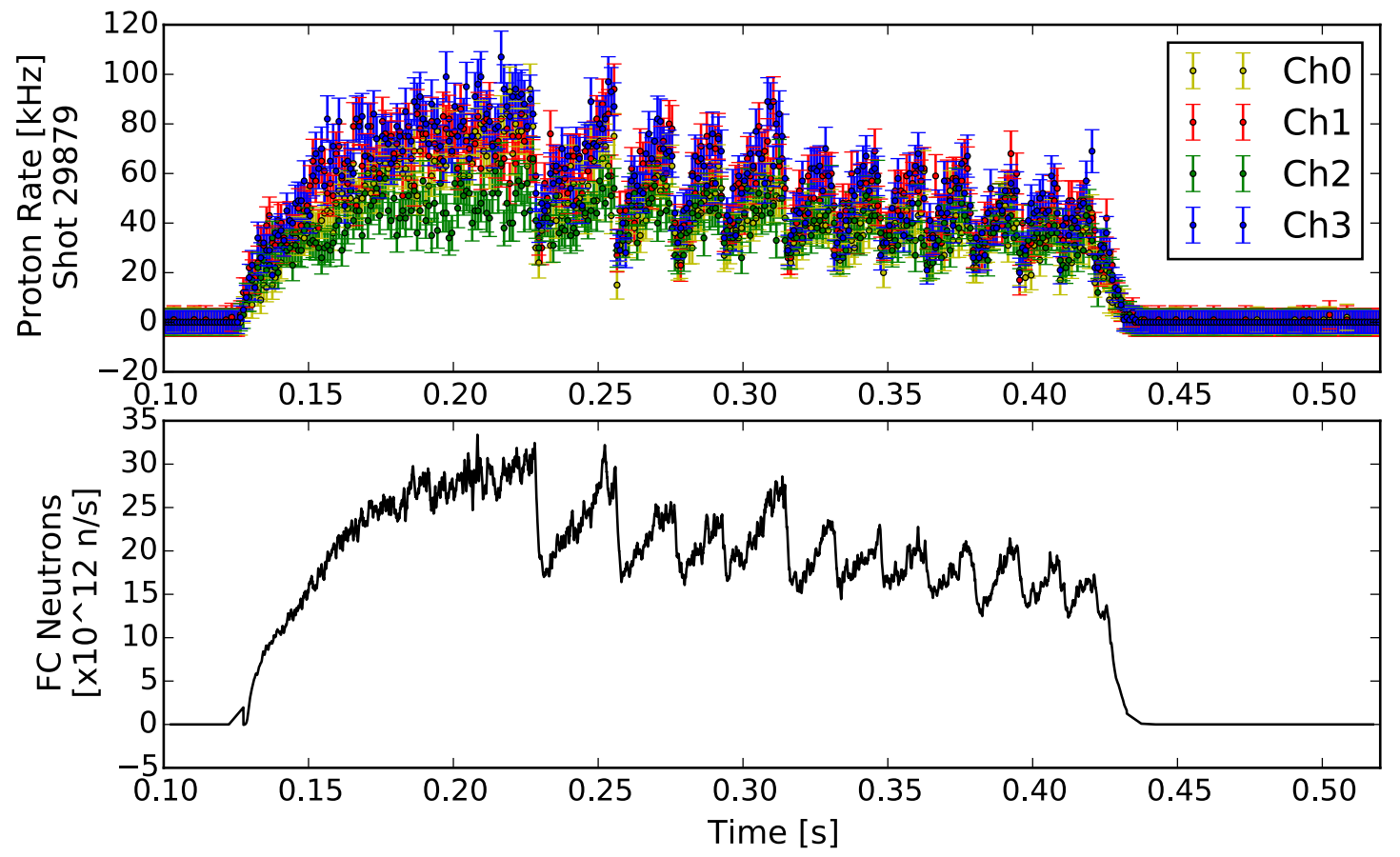

Figure 6.17: Above are data for sawtooth crashes in plasma pulse 29879 from the PD and fission chamber. Data used are from Perez et al., Rev. Sci. Instrum. 85, 11 D701 (2014).[55]

provide complementary information, ${ }^{1}$ however they probe local regions of the plasma using different sightlines. The effect of sawtooth crashes can be observed in local neutron rates measured by the NC and local proton rates measured by the PD in Figure 6.18. Unlike the FC neutron rates, the decrease in rates for the PD and NC vary more drastically between channels; this enables a study of fast ion distributions in local regions of the plasma as opposed to the entire plasma. The trends of the ramping and crashing phases of the sawteeth are seen in both the PD and NC diagnostics. Therefore, for spherical tokamaks with a CND system, the potential to probe different mid-plane radii with these complementary instruments to perform a combined analysis can enhance fast ion loss studies. Additionally, for spherical tokamaks lacking the space for a CND system, such as NSTX-U, the PD can provide experimental data for local variation in DD fusion product rates.

\footnotetext{
${ }^{1}$ Both diagnostics detect products from DD fusion reactions.
} 

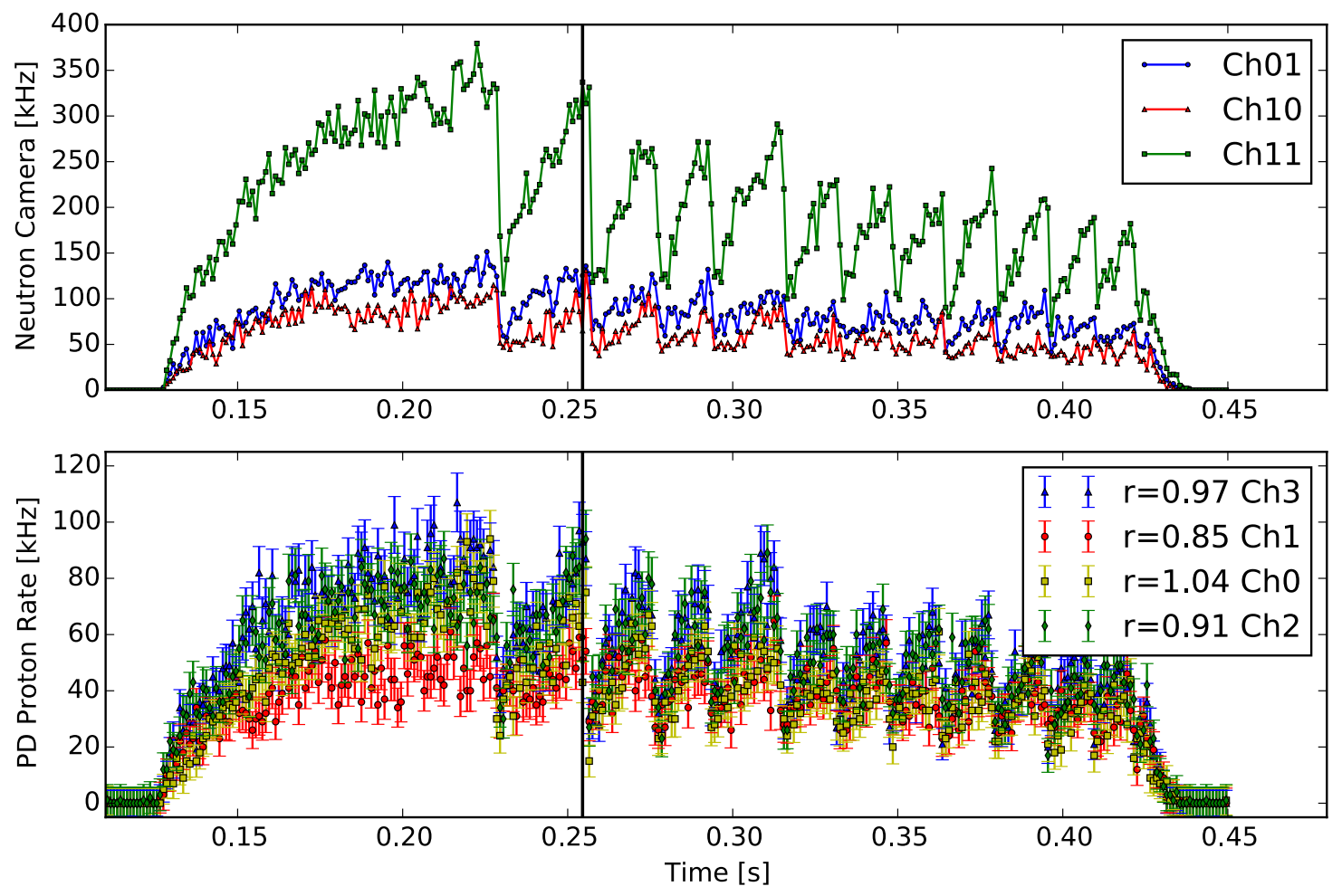

Figure 6.18: Above are data from the Proton Detector and the MAST Neutron Camera during plasma pulse 29879. The units of labeled mid-plane radii are $\mathrm{m}$. The horizontal vertical line indicates the sawtooth crash of interest that is discussed. Caption and data used in Figure are from Perez et al., Rev. Sci. Instrum. 85, 11D701 (2014).[55] 
TRANSP TRANSP is a time dependent energy transport analysis code developed by the PPPL.[60] Results from a TRANSP simulation ${ }^{1}$ in Figure 6.19 show the global proton and neutron rates from DD fusion reactions during NBI heating for a plasma discharge similar to pulse 29879. Similar trends in PD, NC, and FC data can be seen during the crash events.

Additionally, LOCUST is a Monte Carlo computer code which can simulate the full motion of fast ions in the plasma.[71] While LOCUST simulates the full motion of fast ions, TRANSP only simulates the guiding center of fast ion motion throughout the plasma. A future comparison of TRANSP neutron emission rates and LOCUST neutron and proton emission rates, including the radial fast ion distribution in the plasma would enable the evaluation of the accuracy of assuming anisotropic cross sections and simulating guiding centre motion as opposed to full ion gyro-motion.

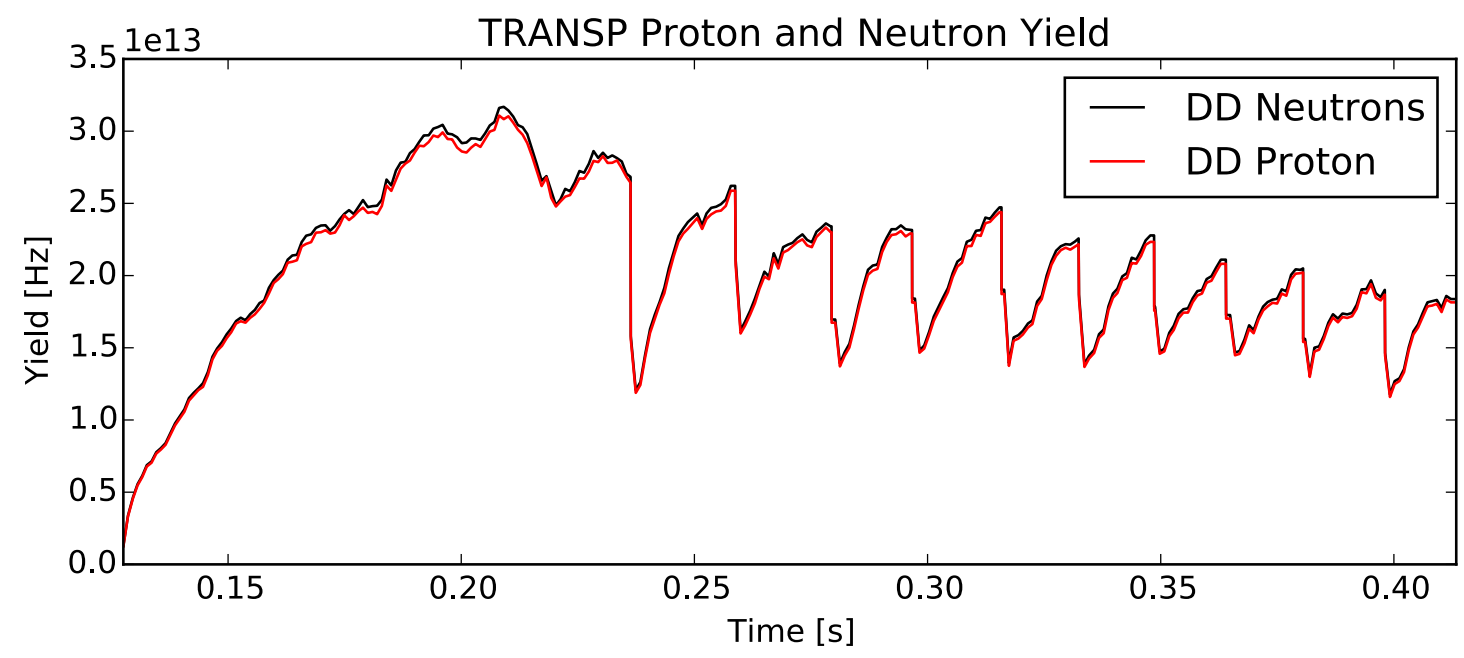

Figure 6.19: Above is a graph of global DD proton and neutron production in the plasma from TRANSP simulation output for a plasma pulse containing sawtooth events (with similar plasma properties as pulse 29879). TRANSP is a time dependent energy transport analysis code developed by the PPPL.[60]

${ }^{1}$ The simulation was run by MAST collaborators. 
Event Emission Profile The second sawtooth crash in Figure 6.18 will be a focus for demonstrating the potential of fitting PD data to create a proton emission profile. Observing proton emission during a sawtooth event allows for the coarse approximation to the radial profile. Integration regions spanning $5 \mathrm{~ms}$ before (249 $\mathrm{ms}$ to $254 \mathrm{~ms})$ and after $(257 \mathrm{~ms}$ to $262 \mathrm{~ms}$ ) the crash are chosen to account for changes in the proton rate. A parameter which can be relevant for the proton emission is the relative magnetic flux, $\psi_{\text {Rel }}$. The relative magnetic flux is defined such that $\psi_{\text {Rel }}=1$ at the magnetic axis and $\psi_{\text {Rel }}=0$ at the plasma edge. The proton emissivity, S, can be fitted to the observed proton rates. $\mathrm{S}$ is integrated along each orbit (for each channel) and the integral is then fitted to the observed experimental proton rate. As each detector contributes only one data point, the small number of parameters yields a model dependent extracted emissivity. ${ }^{1}$ Even so, this initial method makes it possible to observe the general properties of the distribution of S.[55]

Equation (6.44) employs a modulated power law to describe the emissivity $S_{m p}$,

$$
S_{m p}\left(r, z, \psi_{\text {Rel }}\right)=A \psi_{\text {Rel }^{\lambda}}(1+B \cos (\theta))
$$

where $\psi_{\text {Rel }}$ is the relative magnetic flux and $\theta$ is the poloidal angle (a function of $\mathrm{r}$ and $\mathrm{z}$ ). The fitting parameters are A, $\lambda$, and $\mathrm{B} .{ }^{2}$ Note that all of these emissivities have a strong model dependence because there are only four PD data channels. The emissivity function $S_{m p}$ provides a close fit to the radial profile before and after the crash, as seen in Figure

\footnotetext{
${ }^{1}$ Ideally, future versions of the instrument would have more than four data channels, and therefore more data points, to reduce the model dependence of $\mathrm{S}$.

${ }^{2}$ The values of fitting parameters used in the before the crash case are as follows: $A=198766.58+/-2449.25, \lambda=9.47+/-0.16, B=$ $0.45+/-0.008$. The values of fitting parameters used in the after the crash case are as follows: $A=58596.06+/-1599.99, \lambda=4.53+/-$ $0.24, B=0.076+/-0.013$.
} 
6.20. The largest drops in $S_{m p}$, approximately $60 \%$ and $66 \%$, occur near the radial value of the magnetic axis $(0.94 \mathrm{~m})$. The corresponding changes in emission can also be seen in Figure 6.21.

The emissivity $S_{m p}$ is also plotted against $\psi_{\text {Rel }}$. In Figure 6.20 the $S_{m p}$ follows each trajectory during an orbit. The blue line corresponds to Channel 3's orbit in Figure 6.21, green corresponds to Channel 2, red to Channel 1, and yellow to Channel 0 . The emissivity for each channel is double valued. This can be seen while following Channel 3's orbit from the bottom to the top of Figure 6.22, the proton orbit intersects the same $\psi_{\text {Rel }}$ surface at different poloidal coordinate points. It therefore has different values of proton emission (a dispersion or broadening in the emissivity) at different points along the same flux surface. A constant $S_{m p}$ would lie along a single curved line when plotted against $\psi_{\text {Rel. }}$ [55] Broadening in the emissivity before the crash begins at a $\psi_{r e l}$ of approximately 0.6 and after the crash begins at a $\psi_{\text {rel }}$ greater than 0.6 . 


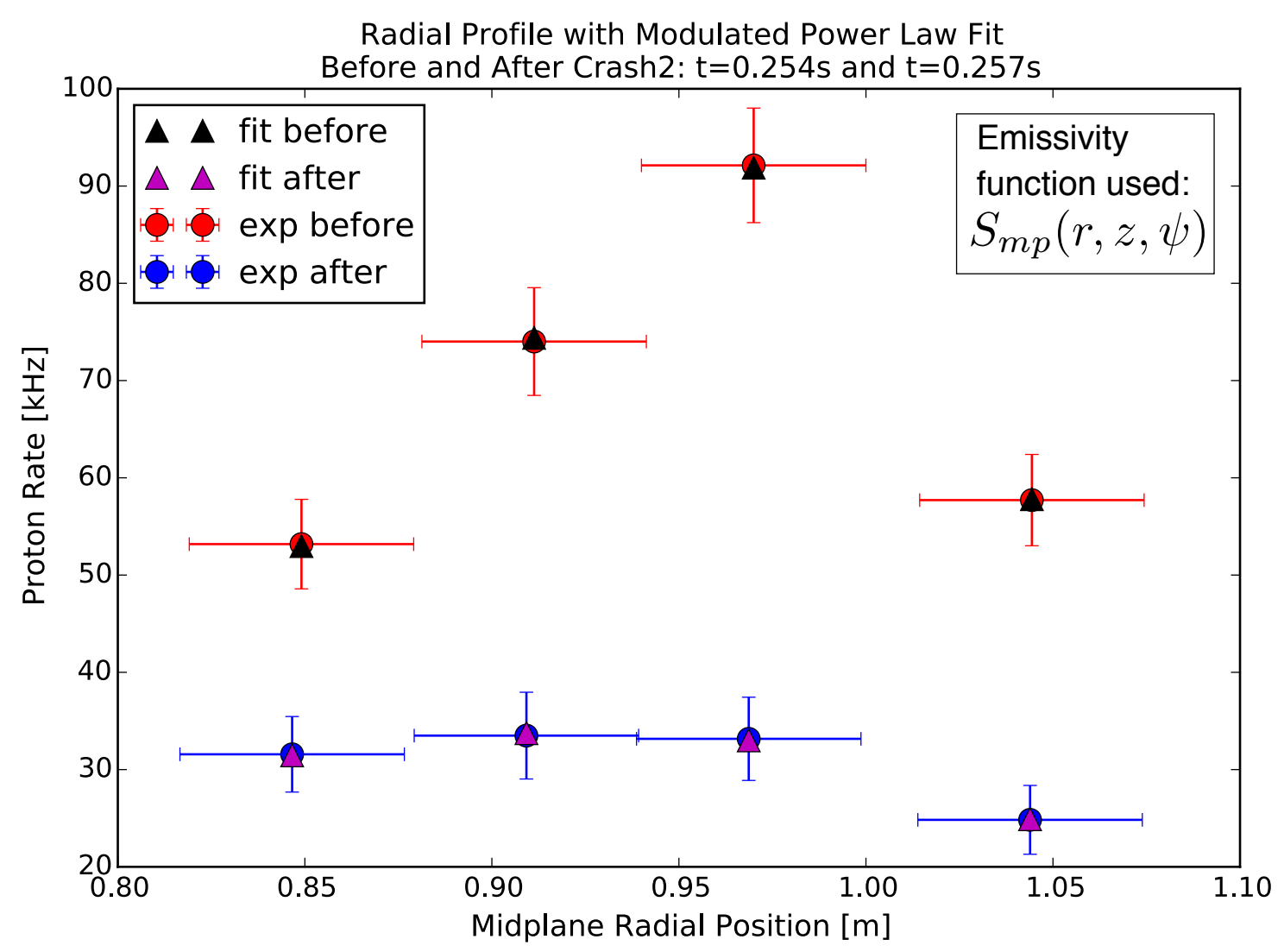

Figure 6.20: Above is the radial emission profile for before and after the second crash in pulse 29879. A modulated power law is used to fit the proton emissivity. The radial value of the magnetic axis is $0.94 \mathrm{~m}$. 

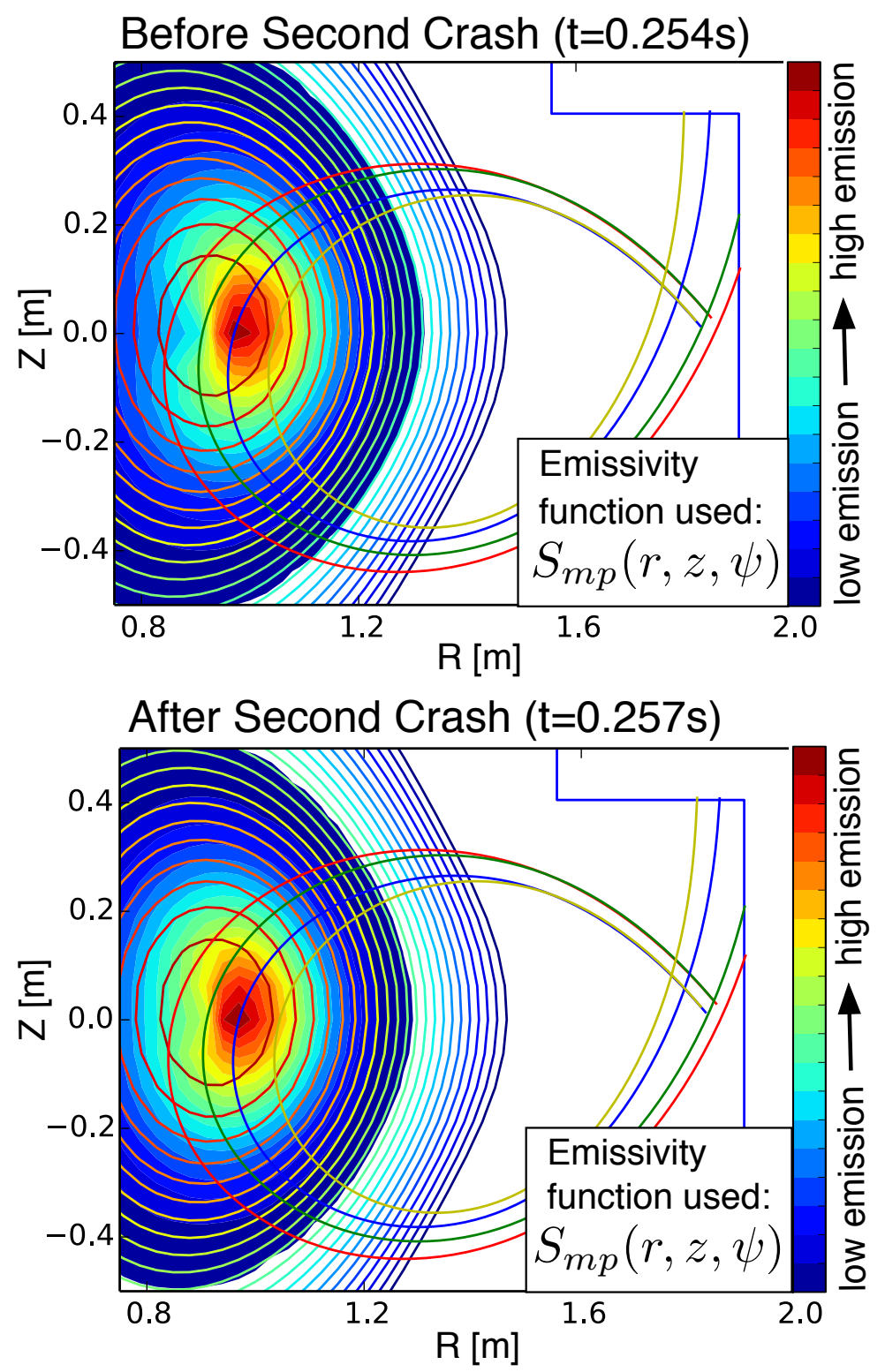

Figure 6.21: The fitted emissivity profile, before and after the second crash, using a modulated power law function for pulse 29879 appear above. The emissivity profile is plotted against the relative magnetic flux surfaces of the plasma. The radial value of the magnetic axis is $0.94 \mathrm{~m}$. 

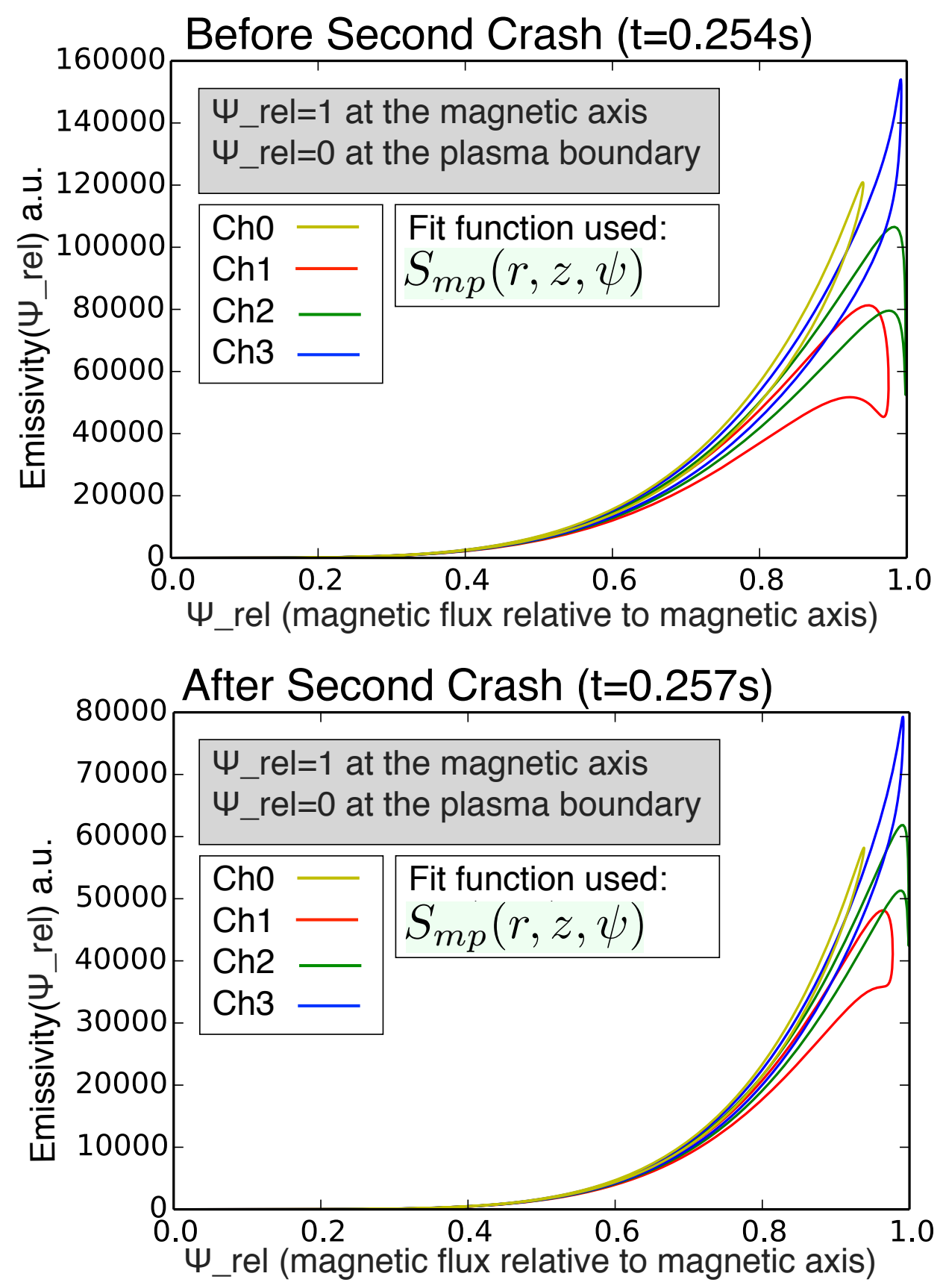

Figure 6.22: Above is the fitted emissivity, based on a modulated power law, for each channel versus the relative magnetic flux. 
A Gaussian function (6.45) can also be used to describe the emissivity, $S_{g}$,

$$
S_{g}(r, z)=\operatorname{Aexp}\left(\left(r-r_{0}\right)^{2}+z^{2}\right) /\left(\sigma^{2}\right)
$$

where $\mathrm{r}$ and $\mathrm{z}$ are positions in the poloidal plane with respect to the magnetic axis. The fitting parameters are A, $r_{0}$, and $\sigma{ }^{1}$ This Gaussian function $S_{g}$ is fitted to the proton emissivity in Figure 6.23.[55] The end time of the $5 \mathrm{~ms}$ time interval used before this crash is $254 \mathrm{~ms}$ and the beginning of the $5 \mathrm{~ms}$ time interval used after this crash is $257 \mathrm{~ms}$. With emissions dropping by nearly half after this crash, the resulting proton emission distribution broadens in Figure 6.24 as the protons redistribute themselves spatially in the plasma.[55] Again, the largest drops in the emissivity occur near the radial value of the magnetic axis $(0.94 \mathrm{~m})$. While the after fit for both models closely represents the experimental data, the before fit for $\mathrm{S}$ is closer to experimental data in $S_{m p}$ rather than $S_{g}$.

The variation of proton emission along a flux surface can be seen in the broadening of the emissivity in Figure 6.24. Additionally Figure 6.24 displays $S_{g}$ in relation to $\psi_{R e l}$ where again the emissivity is not constant throughout an orbit. Broadening in the emissivity before the crash begins at a $\psi_{r e l}$ of approximately 0.6 and after the crash begins at a $\psi_{\text {rel }}$ greater than 0.4. The $S_{m p}$ model (previous model) shows a smaller amount of dispersion in emissivity at a fixed $\psi_{R e l}$ compared to the $S_{g}$ model.

Sawteeth were observed to have an effect on measured proton rates, which followed similar trends to those of measured neutron rates from the MAST neutron camera (NC) and the MAST FC. The drop in the emissivity for data channels after a crash corresponded to the nature of sawteeth oscillations in which temperature fluctuations in the plasma core expel fast ions, thereby reducing fast ion confinement.

\footnotetext{
${ }^{1}$ The values of fitting parameters used in the before the crash case are as follows $A=276122.36+/-51731.090, r_{0}=0.025+/-0.008$, $\sigma=0.134+/-0.016$. The values of fitting parameters used in the after the crash case are as follows: $A=70846.95+/-5481.46, r_{0}=-0.009$ $+/-0.006, \sigma=0.21+/-0.014$.
} 
Radial Profile with Simple Gaussian Fit

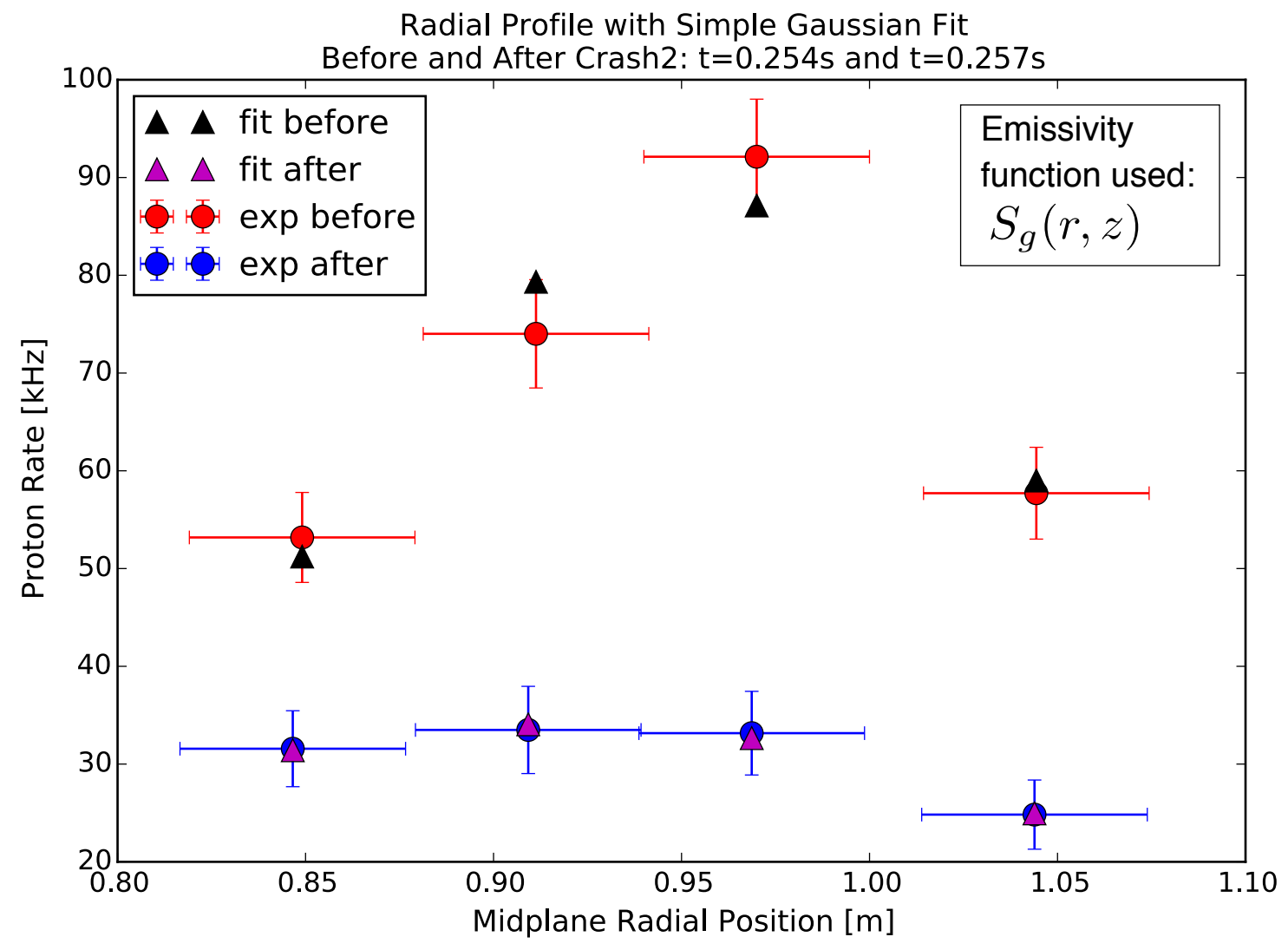

Figure 6.23: Above is the radial emission profile for before and after the second crash in pulse 29879. A modulated power law is used to fit the proton emissivity. The radial value of the magnetic axis is $0.94 \mathrm{~m}$. Figure and Caption are from Perez et al., Rev. Sci. Instrum. 85, 11D701 (2014).[55] 

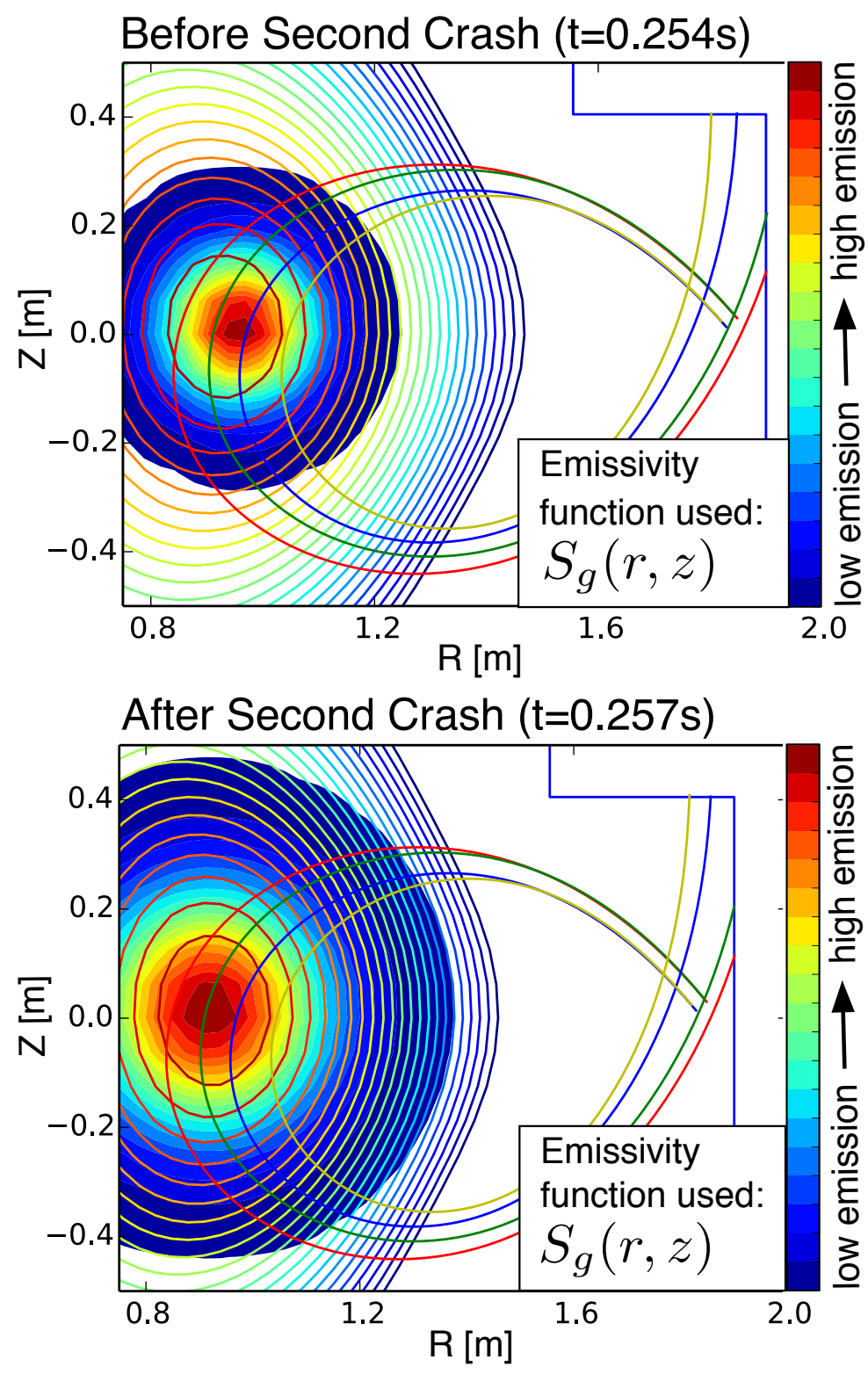

Figure 6.24: The fitted emissivity profile, before and after the second crash, using a Gaussian function for pulse 29879 appear above. The emissivity profile is plotted against the relative magnetic flux surfaces of the plasma. The radial value of the magnetic axis is $0.94 \mathrm{~m}$. Figure and Caption are from Perez et al., Rev. Sci. Instrum. 85, 11D701 (2014).[55] 

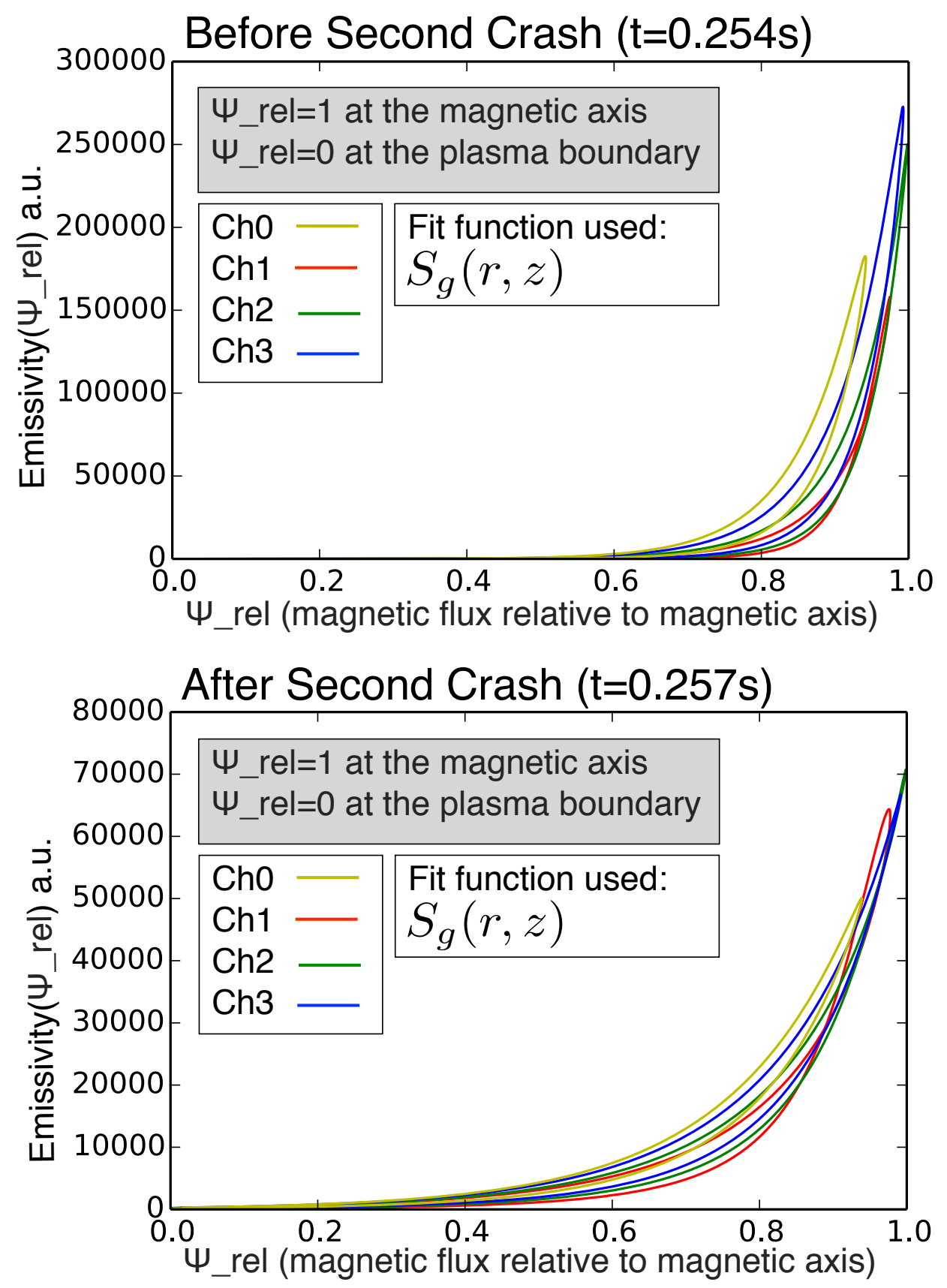

Figure 6.25: Above is the fitted emissivity, based on a Gaussian function, for each channel versus the relative magnetic flux. Figure and caption from Perez et al., Rev. Sci. Instrum. 85, 11D701 (2014).[55] 


\section{CHAPTER 7}

\section{SUMMARY}

\subsection{Charged Fusion Product Diagnostic}

This dissertation work developed a detector system, called the Proton Detector (PD), which was sensitive to emitted $\mathrm{MeV}$ charged fusion products from the deuterium-deuterium (DD) nuclear fusion reaction in (7.1). The PD obtained the first direct measurements of DD 3 $\mathrm{MeV}$ and $1 \mathrm{MeV}$ protons and tritons in a spherical tokamak, specifically in the Mega Amp Spherical Tokamak (MAST).[55]

$$
D+D=P(3.02 M e V)+T(1.01 M e V)
$$

A Monte Carlo simulation using a reconstruction of the plasma's magnetic field, was used to simulate poloidal particle trajectories that would reach the PD. The information was used to design the individual detector orientations such that the trajectories reaching the detectors intersected the plasma core. The PD mechanical design is relatively compact, with a length of $0.2 \mathrm{~m}$ and weight of $4 \mathrm{~kg}$, compared to a collimated neutron detection system, with an approximate length of $2.4 \mathrm{~m}$ and weight of $3600 \mathrm{~kg}$. The non-magnetic steel housing was custom designed for installation into the MAST high vacuum environment. The probe head consisted of a protective thermal shield, four silicon surface barrier detectors, a mechanical filter (aluminum foil), and electrically insulating material. The PD supporting electronics transferred the signals, created by incident particles on the detector, from the vacuum environment to the airside environment where the signals were then amplified, digitized, and stored. 
During 2013, the PD was installed in MAST and collected data during plasma pulses. The data acquisition design and custom written software enabled remote data collection. Pulse-shape functions were fitted to the raw data to extract proton pulses ${ }^{1}$ from shifting baselines and background noise signals. Energy spectra, or histograms, were then created from these fitted pulse amplitudes. Integrating these energy spectra for the energy range of proton signals yielded rates of protons detected after being produced and emitted from the plasma. Energy spectra were created for time intervals as short as $1 \mathrm{~ms}$, which provided the opportunity to observe proton production rate fluctuations on the timescale of plasma oscillations affecting fast ion confinement.

Fast ion relaxation times were calculated for plasma pulses after the neutral beam injection (NBI) heating was turned off. These times were comparable to times calculated from global neutron rates from the MAST fission chamber (FC). These experimental values can be used for comparison to results from Monte Carlo simulation codes such as TRANSP and LOCUST. Additionally, proton rate fluctuations coinciding with fishbone events were observed. The highest fluctuations occurred near the plasma core, which correlates with the effects fishbones have on the plasma, causing the ejections of fast ions from the plasma core. Furthermore, sawteeth oscillations were also observed to have an effect on proton rates, which followed similar trends to those of neutron rates from the MAST neutron camera (NC) and the MAST FC. Falls in PD proton rates and NC neutron rates, as they sampled local regions of the plasma, were more pronounced than changes in the FC global neutron rates, which detected neutrons from the entire plasma. The radial profile for proton emission during one of these sawteeth crashes was constructed. The drop in emission after a crash corresponded to the nature of sawteeth in which temperature fluctuations in the plasma core reduce fast ion confinement.

\footnotetext{
${ }^{1}$ See Chapter 5 for comments regarding triton pulses.
} 
The charged fusion product diagnostic, called the PD, has been successfully designed, constructed, and installed in a spherical tokamak. Analyzed PD data has provided comparable results to established MAST diagnostics and support some expected behavior from plasma oscillations. The potential of this proof of concept instrument to study fast ions emitted from the plasma, and therefore fast ion confinement, has been demonstrated. As more spherical tokamaks are upgraded to higher density plasmas, these types of costeffective and relatively compact diagnostics can positively contribute to studying the effects of oscillations and instabilities using the spatial and temporal distribution of DD fusion reactions.

\subsection{Future Diagnostic Development}

The results from the PD have several implications for fast ion diagnostic development in the experimental plasma physics and fusion research communities. Future versions of the charged fusion product diagnostic consisting of multiple probe heads can be placed into the tokamak such that their crossing curved sightlines would enable a study of any preferential direction of proton and triton emission (see Figure 7.1).

As mentioned in Chapter 1, the first charged fusion product diagnostic was designed for the National Spherical Torus Experiment (NSTX) at the Princeton Plasma Physics Laboratory (PPPL) in Princeton, New Jersey. However, because of an unplanned early shutdown of NSTX to begin upgrades, the instrument was not installed at the NSTX. Consequently the next charged fusion product diagnostic, called the PD, was designed for MAST at the Culham Center for Fusion Energy (CCFE) in Abingdon, the United Kingdom. The third charged fusion product diagnostic is being designed ${ }^{1}$ for the National Spherical Torus Experiment Upgrade (NSTX-U). The motivation of developing a new diagnostic for the

\footnotetext{
${ }^{1}$ Though I have been able to provide materials and input in an advisory role for the new diagnostic's preliminary design, the work for the third diagnostic is being carried out by current and future FIU plasma physics research group members and collaborators.
} 


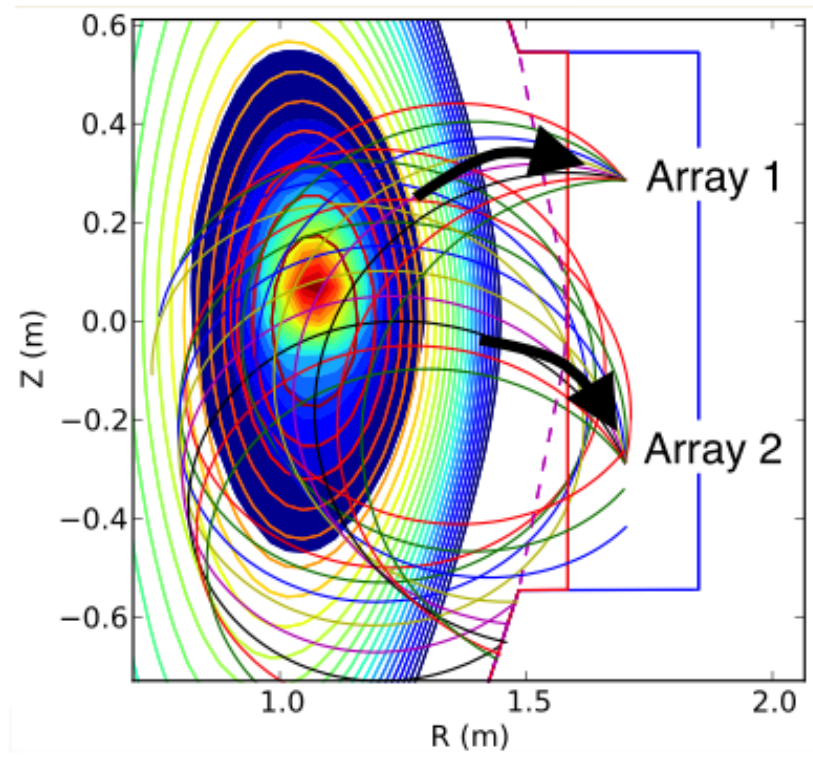

Figure 7.1: Above is an image of two sets of detector array poloidal trajectories projected onto an orbit output simulation. This is a possibility for future PD diagnostics which have more than one probe head inserted into the tokamak.

NSTX-U lies with the relatively compact size of the probe head and supporting electronics. Though it has a fission chamber to measure global neutron rates, the NSTX-U does not have room for a collimated neutron detection system to measure local neutron rates (from which fast ion rates can be extracted). A version of the PD can provide this information for NSTX-U's fast ion confinement studies. Though the motivation of using two probe heads has been stated, this third diagnostic will again comprise only one probe head but will use six detectors as opposed to four. Additionally, to improve signal to noise ratios encountered with the PD, the third diagnostic will use standard coaxial vacuum feedthroughs to transfer signals from the vacuum to the airside environment.

Academic collaborations between FIU, the PPPL, and the CCFE were strengthened through this work. It is expected that this research will provide a basis from which to develop future $\mathrm{MeV}$ charged fusion product diagnostics for spherical tokamaks. 


\section{LIST OF REFERENCES}

[1] Chart from Culham Centre for Fusion Energy Mega Amp Spherical Tokamak parameters: http://www.ccfe.ac.uk/MAST.aspx xii, 5, 6, 9, 11, 12

[2] Culham Centre for Fusion Energy Mega Amp Spherical Tokamak instruments: http://www.ccfe.ac.uk/MAST_diagram.aspx 8

[3] Jeffrey Freidberg, Plasma Physics and Fusion Energy. Cambridge University Press, 2007. xii, 4, 5, 6, 8, 9, 10,11, 12, 13, 104

[4] J. A. Bittencourt, FUNDAMENTALS OF PLASMA PHYSICS. Springer Science + Business Media, LLC, 3rd Edition, 2004. 2

[5] John Wesson, Tokamaks. Oxford University Press, Inc., 2004. xii, 3, 10, 16, 17, 19, $32,34,35,108,109,110,118,128,129$

[6] M. Podesta, W. W. Heidbrink, R. E. Bell, W. Solomon, and V. Soukhanovskii., 49 $9^{\text {th }}$ Annual Meeting of the Division of Plasma Physics Volume 52, Number 11, Poster Session Abstract ID: BAPS.2007.DPP.TP8.87 xii, 5, 6, 9, 12

[7] Jan Mlyna EFD-R(07)01 Focus On: JET, The European Centre of Fusion Research (2007). xii, 5, 6, 9, 12, 18, 19

[8] R. E. Brown and N. Jarmie, Phys. Rev. C 41, 1391 (1990). xiv, 14

[9] Lawrence Livermore National Laboratory: National Ignition Facility \& Photon Science: https://lasers.llnl.gov/science/ignition/ ignition-experiments 1

[10] An image of the spherical tokamak aspect ratio. Copyright CCFE http://www . ccfe.ac.uk/ xiii, 6

[11] Evaluated Nuclear Data File (ENDF) Database http://www.nndc.bnl.gov/ exfor/endf.htm Data Source: Nuclear Energy Agency International Working Party on Evaluation Cooperation, hosted on Server www. nndc.bnl.gov xiv, 13

[12] M. Cecconello, et. al., PPCF-100336.R1, Plasma Phys. Control. Fusion 57 (2015). $11,28,120,124,128,129$

[13] I. Klimek, et. al., Nucl. Fusion 55 (2015). 25, 120, 124

[14] G. Martin, et. al., Physics Scripta. T16, 171 (1987). 22

[15] J. D. Strachan, Rev. Sci. Instrum. 57, 1771 (1986). 22, 30, 35

[16] D. H. Lo, R. L. Boivin, and R. D. Petrasso, Rev. Sci. Instrum. 66, 3453 (1995). 23, 32

[17] R. E. Chrien, R. Kaita, and J. D. Strachan, Nucl. Fusion 23, 1399 (1983). 22 
[18] W. W. Heidbrink and J. D. Strachan, Rev. Sci. Instrum. 56, 501 (1985). 22, 32, 37, 38

[19] M. Cecconello et al., Nucl. Fusion 52, 094015 (2012). xv, 26

[20] M. Cecconello et al., Nucl. Instrum. Methods Phys. Res. A 753, 72837 (2014). 25

[21] H.-S. Bosch, Rev. Sci. Instrum. 61, 1699 (1990). 23, 30, 31, 32, 35, 47

[22] M. Podesta et al., Rev. Sci. Instrum. 79, 10E521 (2008). 24

[23] O. M. Jones et al., Plasma Phys. Control. Fusion 55, 085009 (2013). 24

[24] D. Liu et al., Rev. Sci. Instrum. 77, 10F113 (2006). 24

[25] S. J. Zweben, Rev. Sci. Instrum. 57, 1774 (1986). 22

[26] D.S. Darrow et al., Rev. Sci. Instrum. 66, 476 (1995). 23

[27] D. S. Darrow, R. L. Boivin, and S. J. Zweben, Rev. Sci. Instrum. 63, 4562 (1992). 23

[28] Y. B. Zhu et al., Rev. Sci. Instrum. 83, 10 D304 (2012). 24

[29] Cris W. Barnes and J. D. Strachan, Rev. Sci. Instrum. 61, 2383 (1990). 25

[30] Keith Stammers and M.J. Loughlin. Nuclear Instruments and Methods in Physics Research A 562 (2006). 25

[31] A color image of a typical plasma in the Mega Amp Spherical Tokamak (MAST) fusion device at Culham. Copyright CCFE Composite image made by Scott Silburn, James Harrison, Alex Meakins, and the Culham Centre for Fusion Energy NBI Team.http://www.ccfe.ac.uk/images_detail.aspx?id=8 xiii, 1

[32] Original tokamak field image by Abteilung Offentlichkeitsarbeit (Max-Planck Institut fur Plasmaphysik) [CC BY-SA 3.0 (http://creativecommons.org/licenses/bysa/3.0)], via Wikimedia Commons http://commons.wikimedia.org/ wiki/Fileะ3ATokamak_(scheme).jpg xiii, 9

[33] Figure by Dstrozzi (Own work) [CC $\quad$ BY 2.5 (http://creativecommons.org/licenses/by/2.5)], via Wikimedia Commons. http: // commons.wikimedia.org/wiki/File/\%3AFusion_rxnrate.svg xiii, 5

[34] By Dstrozzi (Own work) [CC BY-SA 3.0 (http://creativecommons.org/licenses/bysa/3.0) or GFDL (http://www.gnu.org/copyleft/fdl.html)], via Wikimedia Commons. http://commons.wikimedia.org/wiki/File\%3AFusion_ tripleprod.svg xiv, 20

[35] MAST cutaway graphic. Copyright CCFE. http://www.ccfe.ac.uk/ images_detail.aspx?id=20 xiii, 7 
[36] EFD-R(07)01 Focus On: JET, The European Centre of Fusion Research (2007). xii, $5,6,9,12,18,19$

[37] Original image JG06.345-1c, EFD-R(07)01 Focus On: JET, The European Centre of Fusion Research (2007). The brochure or parts of it may be reproduced without permission. Text pictures and layout, except where noted, courtesy of the EFDA Parties. The EFDA parties are the European Commission and the Associates of the European Fusion Programme which is coordinated and managed by the commission. Neither the Commission, the Associates nor anyone acting on their behalf is responsible for any damage resulting from the use of information contained in this publication. Additional modifications by Magfuzio group http: //magfuzio.hu/fuzio/kepek/magneses_osszetartas_05.jpg xiv, 21

[38] Image JG03.483-1c, EFD-R(07)01 Focus On: JET, The European Centre of Fusion Research (2007). The brochure or parts of it may be reproduced without permission. Text pictures and layout, except where noted, courtesy of the EFDA Parties. The EFDA parties are the European Commission and the Associates of the European Fusion Programme which is coordinated and managed by the commission. Neither the Commission, the Associates nor anyone acting on their behalf is responsible for any damage resulting from the use of information contained in this publication. xiii, 8

[39] Image JG04.93-8c, EFD-R(07)01 Focus On: JET, The European Centre of Fusion Research (2007). The brochure or parts of it may be reproduced without permission. Text pictures and layout, except where noted, courtesy of the EFDA Parties. The EFDA parties are the European Commission and the Associates of the European Fusion Programme which is coordinated and managed by the commission. Neither the Commission, the Associates nor anyone acting on their behalf is responsible for any damage resulting from the use of information contained in this publication. xiii, 10

[40] Image JG04.93-9c, EFD-R(07)01 Focus On: JET, The European Centre of Fusion Research (2007). The brochure or parts of it may be reproduced without permission. Text pictures and layout, except where noted, courtesy of the EFDA Parties. The EFDA parties are the European Commission and the Associates of the European Fusion Programme which is coordinated and managed by the commission. Neither the Commission, the Associates nor anyone acting on their behalf is responsible for any damage resulting from the use of information contained in this publication. xiii, 2

[41] Image JG03.463-2c, EFD-R(07)01 Focus On: JET, The European Centre of Fusion Research (2007). The brochure or parts of it may be reproduced without permission. Text pictures and layout, except where noted, courtesy of the EFDA Parties. The EFDA parties are the European Commission and the Associates of the European 
Fusion Programme which is coordinated and managed by the commission. Neither the Commission, the Associates nor anyone acting on their behalf is responsible for any damage resulting from the use of information contained in this publication. xiv, 18

[42] J. Felt et al., Rev. Sci. Instrum. 61, 3262 (1990). 30

[43] S. J., Zweben et al., Nucl. Fusion 35, 893 (1995). 32

[44] S. J., Zweben et al., Nucl. Fusion 35, 893 (1995). 32

[45] Hans-Stephan Bosch, Rev. Sci. Instrum. 61, 1699 (1990). 23, 30, 31, 32, 35, 47

[46] D. H. Lo, R. L. Boivin, and R. D. Petrasso, Rev. Sci. Instrum. 66, 3453 (1995). 23, 32

[47] W. W. Heidbrink and J. D. Strachan, Rev. Sci. Instrum. 56, 501 (1985). 22, 32, 37 , 38

[48] W. W. Heidbrink, Ph.D. thesis, Princeton University, 1984. 38

[49] J. D. Strachan, Rev. Sci. Instrum. 57, 1771 (1986). 22, 30, 35

[50] Ortec. Experiment 5 Energy Loss with Heavy Charged Particles (Alphas), Copyright 2010-2014 AMETEK, Inc., http://www.ortec-online.com/ Service-Support/Library/experiments.aspx 47

[51] ASTAR and PSTAR National Institute of Standards and Technology (NIST) Physical Measurements Laboratory data for stopping power and ranges, http:// physics.nist.gov/PhysRefData/Star/Text/ASTAR.html 46

[52] W. K. Chu, Phys. Rev. A, 13, 2057 (1976). 48

[53] J. W. Mayer and E. Rimini. Ion Beam Handbook for Material Analysis. Academic Press, New York, 1977. 47, 48

[54] W.U. Boeglin, R. Valenzuela Perez, and D.S. Darrow, Rev. Sci. Instrum. 81, 10D301 (2010). 35, 128

[55] Perez et al., Rev. Sci. Instrum. 85, 11 D701 (2014). Material, including captions and figures, are reprinted from Perez et al., Rev. Sci. Instrum. 85, 11D701 (2014) with permission. Copyright 2014, AIP Publishing LLC. License Numbers: 3595620628982, 3595620705686, 3595620439451, and 3595620805132. xv, xviii, xix, xx, xxiii, xxiv, 36, 37, 69, 70, 71, 89, 94, 130, 131, 133, 134, 138, 139, 140, 141,142

[56] L. L. Lao et al., Nucl. Fusion 30, 1035 (1990). 31

[57] M. Brix, N.C. Hawkes, A. Boboc, V. Drozdov, and S.E. Sharapov and JET EFDA contributors, Rev. Sci. Instrum. 79, 10F325 (2008). 32 
[58] J. Wesson, Tokamaks. Oxford University Press, Inc., 2004. xii, 3, 10, 16, 17, 19, 32, $34,35,108,109,110,118,128,129$

[59] H. Goldstein, C. P. Poole, and J. L. Safko. Classical Mechanics (3rd edition ed.). Addison-wesley, 2001. 31

[60] R. J. Hawryluk, An Empirical Approach to Tokamak Transport, Physics of Plasmas Close to Thermonuclear Conditions, Proceedings of the Course held in Varenna, Italy (1979) EUR FU BRU/XII/476/80, Commission of the European Communities. Princeton Plasma Physics Laboratory http://w3.pppl.gov/transp/refs xxiii, 132

[61] Computer aided drawing courtesy of the Culham Centre for Fusion Energy Drawing Office. xvi, 41, 42

[62] MAST RP Photo courtesy of and taken by Scott Allan of the Culham Centre for Fusion Energy. xvi, 41

[63] K.G. McClements, S. Sharapov, V. Kiptily, R. Akers, S.Y. Allan, W.U. Boeglin, R.Perez, D.S. Darrow, E. Fredrickson, M. Podesta, L. Roquemore, M. Cecconello, I. Wodniak. Comparison of time-resolved measurements of DD fusion products using proton detector and neutron camera/fission chamber during bursting MHD activity, Approved Proposal. MAST Scientific Programme Proposal for M9 Physics Campaign Experiments. Culham Centre for Fusion Energy, Culham Science Centre, Abingdon, England (2013). 68

[64] D. Keeling, N. Conway, O. Jones, B. Crowley, R. Lake, M. Turnyanskiy, R. Akers, C. Challis, M. Cecconello, I. Wodniak, S. Sangaroon. FPP05, Approved Proposal. MAST Scientific Programme Proposal for M9 Physics Campaign Experiments. Culham Centre for Fusion Energy, Culham Science Centre, Abingdon, England (2013). 68

[65] R. J. Goldston and P. H. Rutherford. Introduction to Plasma Physics. Institute of Physics Publishing, 1995. 16, 17, 104, 105, 106, 107, 108, 109, 110

[66] B. R. Martin. Nuclear and Particle Physics. John Wiley \& Sons Ltd, 2009. xxii, 103, 105

[67] K. G. McClements and M. J. Hole, Physics of Plasmas 19, 072514 (2012). 109

[68] R. J. Akers et. al., Session EXP4/19. $18^{\text {th }}$ Fusion Energy Conference (IAEA-CN-77), International Atomic Energy Agency and Italian National Agency for New Technology, Energy, and the Environment. Sorrento, Italy (2001). 110

[69] M. R. Tournianski, R. J. Akers, P. G. Carolan and D. L. Keeling, Plasma Phys. Control. Fusion 47, 671 (2005). 109, 110, 114

[70] A. Fasoli et. al., Nucl. Fusion 47, S264S284 (2007). 118, 119 
[71] Akers et. al., Nucl.Fusion 42, 122 (2002). 132 
VITA

RAMONA L. V. PEREZ

EDUCATION AND HONORS

2003-2008

2008-2014

2014-2015

2014

2006-2015

2003-2005
B.S., Physics

Florida International University, Miami, FL

Graduate Research and Teaching Assistant Physics Department

Florida International University

Miami, FL

FIU Dissertation Year Fellowship Award

American Physical Forum on Graduate Student Affairs

Travel Award for Excellence in Graduate Research

Ronald E. McNair Baccalaureate Program Fellow

FIU Presidential \& Salutatorian Scholarship

\section{PUBLICATIONS AND PRESENTATIONS}

W.U. Boeglin, R. Valenzuela Perez, D.S. Darrow. Concept of a charged fusion product diagnostic for NSTX. Rev. Sci. Instrum. 81, 10D301 (2010).

W.U. Boeglin and R.V. Perez. Charged Fusion Product Diagnostic Final Design Review, Princeton Plasma Physics Laboratory, Princeton, NJ (04/2011)

R.V. Perez and W.U. Boeglin. Charged Fusion Product Diagnostic Mechanical Design Review, Culham Centre for Fusion Energy, Culham Science Centre, Abingdon, England (09/2012).

R.V. Perez, S.Y. Allan, W.U. Boeglin, M. Cecconello, K.G. McClements, D.S. Darrow, and the MAST team. First Results from a Charged Fusion Products Diagnostic at MAST, Poster session, APS DPP 55T H Annual Meeting. Denver, Colorado (11/2013) 
R.V. Perez. Charged Fusion Product Diagnostic Electrical Design Review, Culham Centre for Fusion Energy, Culham Science Centre, Abingdon, England (05/2013).

R.V. Perez. Initial Results from the Proton Detector, Friday Physics Seminar. Culham Centre for Fusion Energy, Culham Science Centre, Abingdon, England (09/2013).

R.V. Perez, W.U. Boeglin, D.S. Darrow, M. Cecconello, I. Klimek, S.Y. Allan, R.J. Akers, D.L. Keeling, K.G. McClements, R. Scannell, M. Turnyanskiy, A. Angulo, P. Avila, O. Leon, C. Lopez, O.M. Jones, N.J. Conway, and C.A. Michael. Investigating Fusion Plasma Instabilities in the Mega Amp Spherical Tokamak Using MeV Proton Emissions, Invited Talk, The 20TH Topical Conference on High Temperature Plasma Diagnostics. Atlanta, Georgia (06/2014).

R.V. Perez, W.U. Boeglin, D.S. Darrow, M. Cecconello, I. Klimek, S.Y. Allan, R.J. Akers, D.L. Keeling, K.G. McClements, R. Scannell, M. Turnyanskiy, A. Angulo, P. Avila, O. Leon, C. Lopez, O.M. Jones, N.J. Conway, and C.A. Michael. Investigating fusion plasma instabilities in the Mega Amp Spherical Tokamak using mega electron volt proton emissions, Invited Paper. Rev. Sci. Instrum. 85, 11D701 (2014). Figure 2 selected as cover art for HTPD 2014 Conference Proceedings.

M. Cecconello, O.M. Jones, W.U. Boeglin, R.V. Perez, D.S. Darrow, I. Klimek, S.E. Sharapov, M. Fitzgerald, K.G. McClements, D.L. Keeling, S.Y. Allan, C.A. Michael, R.J. Akers, N.J. Conway, R. Scannell, M. Turnyanskiy, G. Ericsson \& the MAST team. Energetic ion behaviour in MAST. PPCF-100336.R1, Plasma Phys. Control. Fusion 57 (2015). 